Horst Bredekamp und Wolfgang Schäffner (Hg.)

Haare hören - Strukturen wissen - Räume agieren 

Horst Bredekamp und Wolfgang Schäffner (Hg.)

\section{Haare hören - Strukturen wissen - Räume agieren}

Berichte aus dem Interdisziplinären Labor Bild Wissen Gestaltung

Jahrestagung, 15. November 2014, in der Berlin-Brandenburgischen Akademie der Wissenschaften 
Die Publikation wird ermöglicht durch die finanzielle Unterstützung der Deutschen Forschungsgemeinschaft im Rahmen der Exzellenzinitiative.

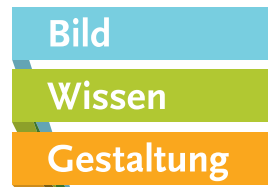

Ein Interdisziplinäres Labor
Gefördert durch die

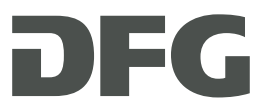

Dieses Werk ist lizensiert unter der Creative Commons Attribution-NonCommercial-NoDerivs 3.0 DE Lizenz. Diese Lizenz erlaubt die private Nutzung, gestattet aber keine Bearbeitung und keine kommerzielle Nutzung.

Weitere Informationen finden Sie unter http://creativecommons.org/licenses/by-nc-nd/3.0/de

\section{Bibliografische Information der Deutschen Nationalbibliothek}

Die Deutsche Nationalbibliothek verzeichnet diese Publikation in der Deutschen Nationalbibliografie; detaillierte bibliografische Daten sind im Internet über http://dnb.d-nb.de abrufbar.

\section{(C) 2015 transcript Verlag, Bielefeld}

Die Verwertung der Texte und Bilder ist ohne Zustimmung des Verlages urheberechtswidrig und strafbar. Das gilt auch für Vervielfältigungen, Übersetzungen, Mikroverfilmungen und für die Verarbeitung mit elektronischen Systemen.

Layout und Satz: Kerstin Kühl, Berlin

Umschlagabbildung und Kapitelopener: Kerstin Kühl, Berlin 2014

Redaktion: Kerstin Germer und Maja Stark | Bild Wissen Gestaltung

Printed in Germany

Print-ISBN 978-3-8376-3272-9

PDF-ISBN 978-3-8394-3272-3

Gedruckt auf alterungsbeständigem Papier mit chlorfrei gebleichtem Zellstoff.

Besuchen Sie uns im Internet: http://www.transcript-verlag.de

Bitte fordern Sie unser Gesamtverzeichnis und andere Broschüren an unter:

info@transcript-verlag.de 



\section{Inhaltsverzeichnis}

S. 8 Vorwort

\section{Bilderwissen}

S.13 Bilderwissen im Zeitalter des Neomanierismus

Horst Bredekamp

S. 31 Im Bildlabor der Neurochirurgie. Ein interdisziplinärer bildgeführter Fallbericht Kathrin Friedrich, Thomas Picht, Moritz Queisner und Anna L. Roethe

S. 45 Wissenschaftliche Sachcomics. Multimodale Bildsprache, partizipative Wissensgenerierung und raumzeitliche Gestaltungsmöglichkeiten Reinhold Leinfelder, Alexandra Hamann und Jens Kirstein

S. 61 Morphologische Analysen vegetabiler Ornamente der Gotik. Eine interdisziplinäre Annäherung an kunsthistorische Reihen

Anja Seliger, Günther Jirikowski und Gerhard Scholtz

S. 79 Wahrnehmung von Datengrafiken. Ein verzerrter Eindruck?

Claudia Godau und Robert Gaschler

\section{Wissensstrukturen}

S. 89 Die Entdeckung einer wissensarchitektonischen Karte

Sabine Hansmann und Peter Koval

S.103 Im Umkreisen begriffen. Über die Produktivität kommunikativer Ambiguität Christian Stein

S.119 mauern. Wissensstruktur und Strukturwissen der Architektur Michael Dürfeld

S.133 Zu einer Unschärferelation der Modelle. Präzision und Produktivität mehrdeutiger Modelle in der Gestaltung

Reinhard Wendler 


\section{Gestaltung als Synthese}

S. 147 Wissen - Nichtwissen - Entwerfen

Philipp Oswalt

S.159 Entwerfen - Verwerfen. Ein reflektierender Werkstattbericht aus dem Interaction Design

Anouk-Aimée Hoffmeister

S. 171 Mit den Haaren hören. 1832-2014-1897

Christian Kassung, Sebastian Schwesinger und Christian Seifert

S.185 Bilder der Bewegung. Sexualität im Zellkern um 1900 und nach 2000 Bettina Bock von Wülfingen

S.199 Interdisziplinäre Gestaltung. Einladung in das neue Feld einer Geistesund Materialwissenschaft

Wolfgang Schäffner

S. 214 Abbildungsnachweis 


\section{Vorwort}

Die Exzellenzinitative hat die Forschungs- und Lehrstrukturen in Deutschland stark verändert. Zwei Jahre nach dem großen Presseecho, das mit der Bewilligung der zweiten Runde 2012 einherging, nutzte das Interdisziplinäre Labor Bild Wissen Gestaltung die Gelegenheit seines zweijährigen Bestehens, über die Einlösung seiner Ziele wie auch über die Problemstellungen anhand ausgewählter Vorhaben zu unterrichten.

Ausgerichtet am 15. November 2014 in der Berlin-Brandenburgischen Akademie der Wissenschaften, konzentrierte sich die erste Jahrestagung des Exzellenzclusters der Humboldt-Universität zu Berlin auf seine drei titelgebenden Schlagwörter, um Vorträge in den Sektionen Bilderwissen, Wissensstrukturen und Gestaltung als Synthese zu präsentieren.

Der vorliegende Tagungsband nimmt diese Trias auf. Er versammelt in drei Kapiteln ein weites Spektrum an Beiträgen von Wissenschaftlerinnen und Wissenschaftlern des Clusters, die in ihrer Diversität die Vielfalt der dort betriebenen Forschungen widerzuspiegeln versuchen.

Die neuen Bildtechniken in den Wissenschaften bilden den Ausgangspunkt für Fragestellungen, die ihren Fokus nicht länger auf die epistemische Bestimmung dessen setzen, was ein Bild ist. Vielmehr werden unterschiedliche Wissensprozesse und Praktiken in den Vordergrund gerückt, in denen Bilder und bildgestützte Verfahren eine elementare Rolle spielen. Unter dem Titel Bilderwissen stellt das erste Kapitel nach einem einführenden Versuch einer Diagnose unserer Zeit (Horst Bredekamp) exemplarisch die Möglichkeiten und Probleme des Methodentransfers im Exzellenzcluster vor. So werden Methoden der morphologischen Biologie eingesetzt, um vegetabile Ornamentsysteme der Gotik und deren Transformationen zu untersuchen (Anja Seliger, Günther Jirikowski, Gerhard Scholtz). Anhand einer gemeinsamen Falldokumentation in der Neurochirurgie verdeutlichen Medienwissenschaft und Medizin, wie Bilder ärztliche Handlungen und Entscheidungen bestimmen (Kathrin Friedrich, Thomas Picht, Moritz Queisner, Anna L. Roethe). Zudem wird in diesem Kapitel die aktiv gestaltende Rolle von Bildern im Forschungsprozess analysiert, die einerseits in ihrer Wahrnehmung Verzerrungen unterliegen können (Claudia Godau, Robert Gaschler) und andererseits ein wichtiges, historisch unterschätztes Instrument zur Wissensvermittlung darstellen (Reinhold Leinfelder, Alexandra Hamann, Jens Kirstein).

Das Interdisziplinäre Labor verbindet Wissen aus mehr als 25 Disziplinen der Geisteswissenschaften, Naturwissenschaften und Gestaltung. Mit Blick auf die digitale Revolution und die Umwälzung auf der Ebene der Materialien denkt es Wissensstrukturen in ihrer Gesamtheit neu. Ihnen ist das zweite Kapitel gewidmet, das die interdisziplinäre Zusammenarbeit als Prozess und Forschungsgegenstand des Clusters vorstellt: Das Live-Erlebnis einer Lecture Performance, die im Rahmen der Tagung den Austausch, die Kontroverse und die Übereinkunft zwischen 
dem Wissen der Architektur und der Kulturwissenschaft präsentierte, wird verschriftlicht im Minutentakt wiedergegeben (Sabine Hansmann, Peter Koval). In einem weiteren Beitrag werden gelingende und scheiternde Kommunikationen im Interdisziplinären Labor analysiert (Christian Stein). Erkenntnisse der Selbstbeobachtung fächerübergreifender Forschung werden in den Exzellenzcluster zurückgespiegelt, um die Bedingungen für problemorientierte interdisziplinäre Zusammenarbeit kontinuierlich zu verbessern. Die einzelnen Disziplinen werden bei dieser Vorgehensweise keinesfalls negiert, sondern gerade mit ihren teils radikal unterschiedlichen Methoden in die interdisziplinäre Arbeit integriert. Daher präsentiert das zweite Kapitel auch die disziplinären Erträge dieses Zusammenspiels. Die Architektur fragt vor dem Hintergrund neuer Hochtechnologie im Bau, was sie mittels eigener Methoden und Praktiken zu einem interdisziplinären Strukturwissen beitragen kann (Michael Dürfeld), und die Kunstgeschichte befasst sich mit dem Potenzial mehrdeutiger Modelle in der Gestaltung (Reinhard Wendler).

Gestaltung als integrativer Akt bildet im Exzellenzcluster ein zentrales Verfahren, das unterschiedlichste Wissensformen miteinander verbindet. Architektur und Design sind im Interdisziplinären Labor aktiv in die Grundlagenforschung involviert, um den Wissenschaften neue Potenziale durch Gestaltung zu eröffnen. So kann die Frage nach den gemeinsamen Praktiken von Bildgebung und Wissenserzeugung auf die Thematik ihres Gestaltungspotenzials zugespitzt und gleichzeitig Wissen in neue Gestaltungsprozesse überführt werden. Dieses programmatische Verständnis von Gestaltung als Synthese wird im dritten Kapitel erläutert. Exemplarisch werden konkrete interdisziplinäre Gestaltungsprojekte präsentiert. So forschen Physik und Kulturwissenschaft gemeinsam zu einem neuen Analogspeicher, einem extrem verkleinerten Selbstschreiber auf Nanoebene (Christian Kassung, Sebastian Schwesinger, Christian Seifert). Entwurfs- und Fertigungsverfahren des Interaction Designs bewegen sich zwischen Entwerfen und Verwerfen, um schließlich in konkrete Werkzeuge für die interdisziplinäre und vernetzte Forschung einzufließen (Anouk-Aimée Hoffmeister). Gestaltung wird zudem als Synthese begriffen, die besonders dann produktiv ist, wenn disziplineigene Differenzen zwischen Wissenschaft und Gestaltung nicht nivelliert, sondern im Gegenteil gewahrt werden (Philipp Oswalt). Der abschließende Beitrag zur interdisziplinären Gestaltung (Wolfgang Schäffner) lädt aus der historischen Rückschau in das neue Feld einer Geistes- und Materialwissenschaft ein. Demnach sind nicht nur gestalterische Prozesse in der technisch hochgerüsteten Wissensgesellschaft als genuine Forschungsleistung zu begreifen; vielmehr kann auch Forschung als Gestaltungsprozess verstanden werden. Als Ergebnis von einem in diesem Sinne erweiterten Forschungs- und Gestaltungsprozess, den das Interdisziplinäre Labor Bild Wissen Gestaltung in Gang setzt, darf auch der vorliegende Tagungsband betrachtet und gelesen werden.

Berlin, im Frühjahr 2015

Horst Bredekamp und Wolfgang Schäffner 


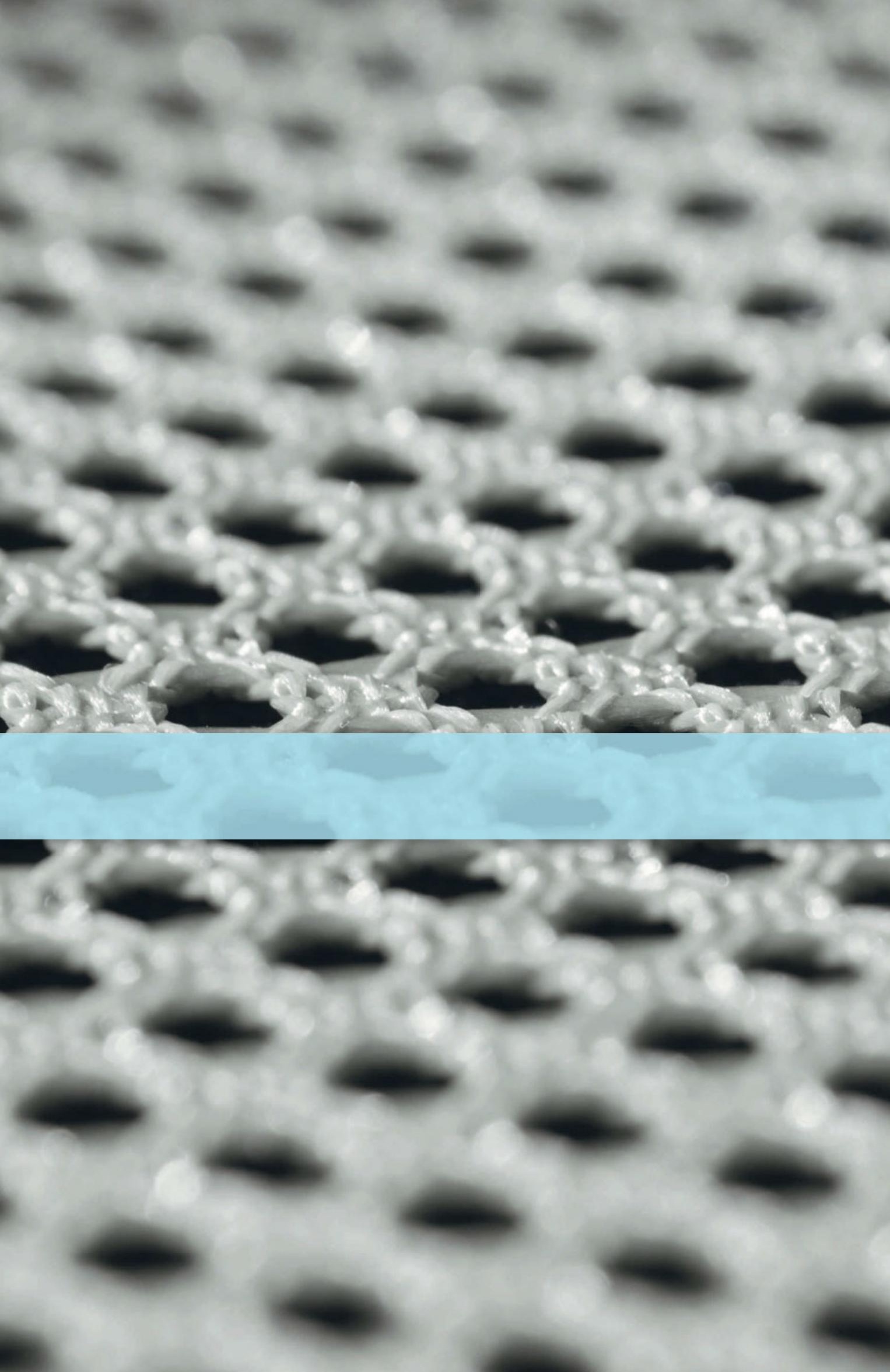





\section{Bilderwissen im Zeitalter des}

\section{Neomanierismus}

\section{Die Wiederkehr des Manierismus}

Die innerlich verbundene Trias der Begriffe Bild, Wissen und Gestaltung legt in ihrer Komplexität etwas nahe, das angesichts der scheinbaren Unübersichtlichkeit der Gegenwart in der Regel kategorisch ausgeschlossen wird: eine Theorie der eigenen Zeit. Aber so sicher es ist, dass kein Bild der Zeit entworfen werden kann, das umfassenden Charakter besitzt, so unabweisbar ist es, dass ohne den Versuch einer Theorie das Handeln ohne Orientierung bleibt.

Frühere Zeiten, die nicht weniger unübersichtlich erschienen, waren eher bereit, den Versuch einer panoramatischen Orientierung zu unternehmen. Als Modell kann eines der bedeutendsten Werke der Literaturwissenschaft gelten, Ernst Robert Curtius' Europäische Literatur und lateinisches Mittelalter. In diesem im Jahr 1948 erschienenen Opus hat Curtius die nachantiken Kulturbewegungen als Pendelschläge zwischen klassischer Norm und manieristischem Normensprung beschrieben. Der Manierismus betrifft die zwischen Renaissance und Barock liegende Zeitspanne der Jahrzehnte von 1530 bis 1620, die ihre Bezeichnung im 18. Jahrhundert erhalten hat. Sie entstand aufgrund der Annahme, dass die Finesse der künstlerischen Hand, die maniera, alle Grenzen überwinden könne: die zwischen Bild und Körper, Natur- und Kunstform sowie zwischen Innen und Außen. ${ }^{2}$

1 Curtius 1948.

2 Siehe Bredekamp 2000; Huss/Wehr 2014. 


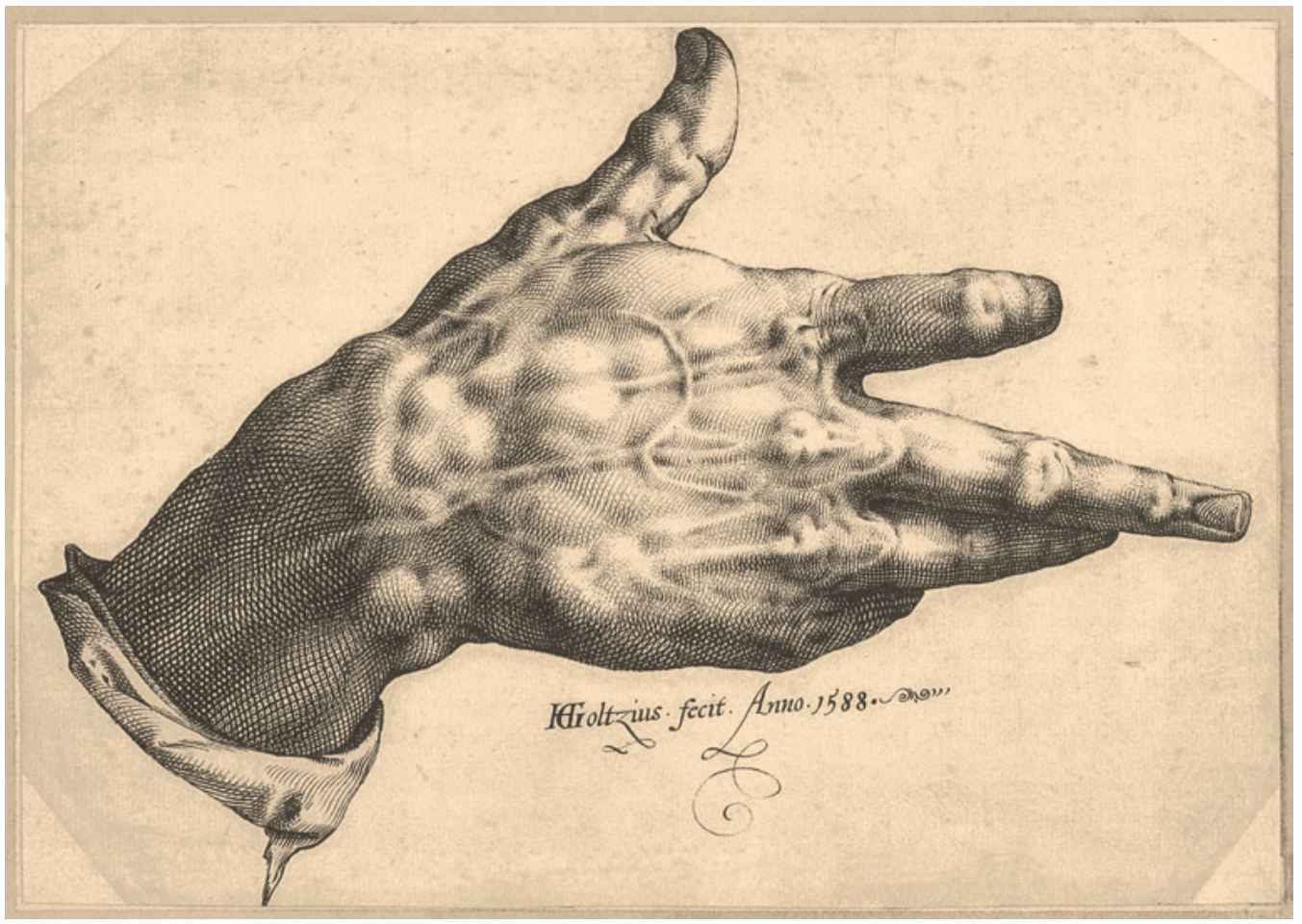

Abb. 1: Hendrick Goltzius, Die rechte Hand des Künstlers, 1588, Feder in Braun, 23 × 32,2 cm, Haarlem, Tylers Museum.

Manier und Manierismus sind in dieser ursprünglichen Bestimmung keineswegs negativ konnotiert, sondern mit höchster Wertschätzung versehen. Dem heute abwertend gemeinten Adjektiv >manieriert< steht das historische Verständnis des Manierismus als Epoche der Kunstfertigkeit der Hand, manus, entgegen. Immer wieder verblüfft es, wie es ihr gelingen konnte, die Gattungsgrenzen zu überspielen. Es geht um ein Können, das vor keiner Grenze haltmacht, so auch nicht vor den Unterschieden zwischen individueller Linie und Maschinenkunst, wie sie die Linienapparate der Drucktechniken hervorbrachten. ${ }^{3}$

Das um 1580 geschaffene Selbstporträt des niederländischen Künstlers Hendrik Goltzius in Form seiner eigenen Linken besticht nicht nur durch die skrupulöse Wiedergabe ihrer teils verknorpelten Binnenstruktur und die Hervorhebung der unter der Haut liegenden Adern, sondern auch durch die Präzision der parallel geführten Linien der Oberfläche, die das Verfahren des 
Tiefdrucks zeigen (Abb. 1). ${ }^{4}$ Die Genauigkeit dieser schwellenden und sich kontinuierlich aufeinander zu- und wegbewegenden Linien lassen eine maschinelle Ritzung der Kupferstichplatte vermuten. Aber dies ist eine Illusion. Die Hand ist unfassbarerweise nicht gedruckt, sondern gezeichnet. Der Künstler hat im Medium des disegno die maschinelle Drucktechnik aufgehoben. Es handelt sich um eine kaum jemals wieder erreichte Höchstleistung des Zusammenspiels zweier differenter Techniken und Medien. Die Drucktechnik treibt ihr Gegenüber dazu an, sie zu übertreffen, womit eine Wertschätzung ihrer selbst im Medium ihrer Übertrumpfung entsteht: als Spirale eines mitstreitenden Paragone. ${ }^{5}$

In einem solch neutralen Verständnis erscheint die Epochenbezeichnung des Manierismus als ein besonderes Signum auch unserer Zeit, zumal sie über Curtius' Schüler Gustav René Hocke und ihm vorausgehende Autoren ebenso die Sensibilität für das Krisenhafte und Zerrissene einschloss. ${ }^{6}$ In seiner Witterung für alles Gefährdete konnte der Manierismus solcherart als Zeichen einer wagemutig diagnostischen Moderne gelten. Und wenn es ein Motiv gibt, das auf unsere Epoche gleichfalls zutreffen könnte, so ist es dieses Klima einer kulturell, militärisch und finanziell erkannten oder zumindest allgemein gefühlten Bedrohung.

Die bisher vorgelegten Analysen des Manierismus und seiner Wiederkehr waren vornehmlich auf Kunst und Literatur fokussiert, bezogen aber nicht minder alle Formen der Naturforschung und Technologie mit ein. ${ }^{7}$ Es ist nicht zuletzt diese umfassende Geltung, die ihn als so gegenwärtig erscheinen lässt. Aus diesem Grund ist seine hypostasierte Wiederkehr in all ihrer Produktivität, aber auch in ihrem problematischen Potenzial gleichermaßen als ein gesamtkulturelles Phänomen zu beschreiben. Die Gegenwart unter den Begriff des Neomanierismus zu stellen, erscheint daher als eine Möglichkeit, Phänomene zu beleuchten, die ihre innere Bindung auf andere Weise nicht sichtbar werden lassen. Es ist gleichwohl bezeichnend, dass ungeachtet oder gerade wegen dieser unbewusst oder gezielt wirkenden Verbindung unserer Zeit mit dem Manierismus die aktuelle bildende Kunst als gemalte Theorie auftritt, als Bilderwissen.

4 Siehe Leeflang et al. 2003, 244f. Bettina Uppenkamp aus dem Interdisziplinären Labor bereitet hierzu eine Publikation vor.

5 Siehe Gastel/Hadjinicolaou/Rath 2014.

6 Siehe Hocke 1957. Zur früheren Literatur siehe Bredekamp 2000.

7 Siehe Battisti 1962. 
Abb. 2: Michael Triegel, Verwandlung der Götter, 2010, Mischtechnik auf MDF, $196 \times 117 \mathrm{~cm}$, Erfurt, Angermuseum.

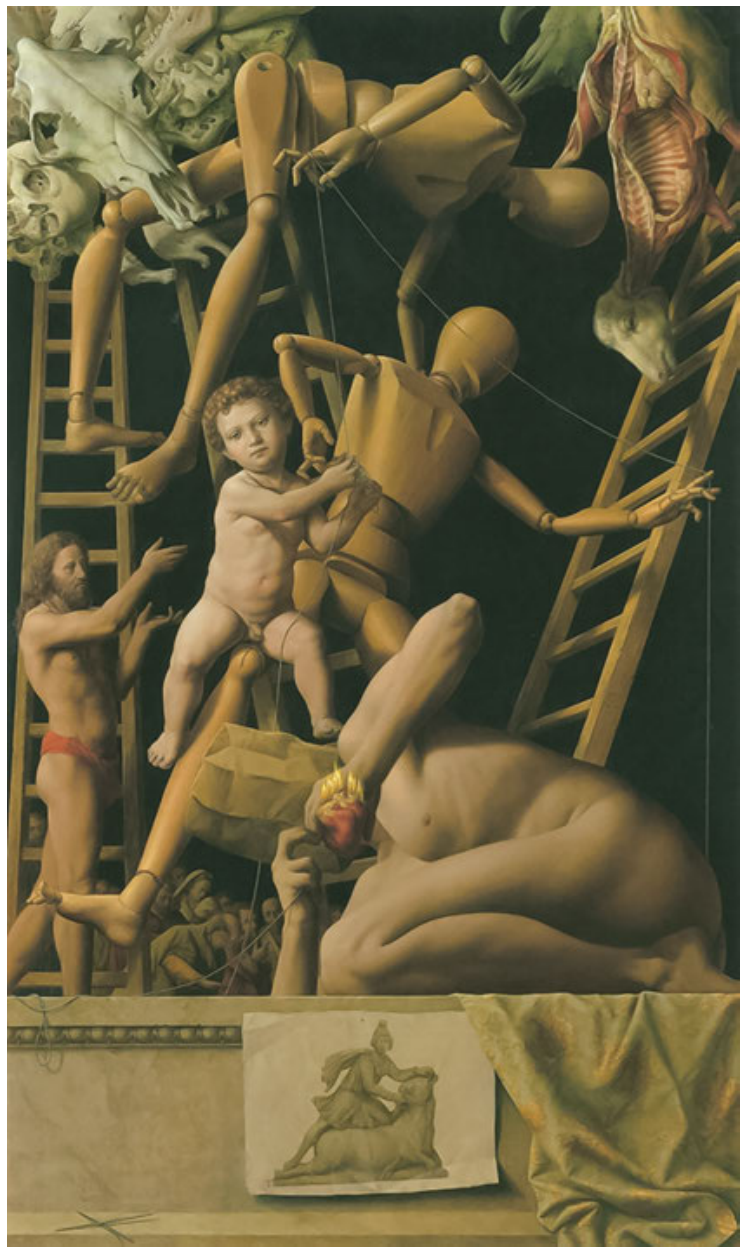

\section{Manieristische Motive}

Das Doppelspiel zwischen Rückgriff auf den Manierismus und Zeitbezug gilt in besonderer Weise für den Leipziger Maler Michael Triegel. ${ }^{8}$ Deutlich wird dies vor allem an seinem Gemälde Verwandlung der Götter (Abb. 2), ${ }^{9}$ das eine Kreuzabnahme zeigt, bei welcher der obere Scherge wie auch die Christusgestalt als lebendige, historisch als mannequinos bezeichnete Holzfiguren erscheinen, wie sie in Künstlerateliers noch immer verwendet werden. Der zeitliche Rückbezug wird im Vergleich der Verwandlung der Götter mit einer um 1560 entstandenen Zeichnung Luca Cambiasos offensichtlich (Abb.3), welche die Körper zu Formen abstrahiert hat, die auf den

8 Triegel ist dadurch bekannt geworden, dass er Papst Benedikt XVI. in einem Staatsporträt verewigt hat, siehe Hüttel 2014,155 .

9 Siehe ebd., 163. 


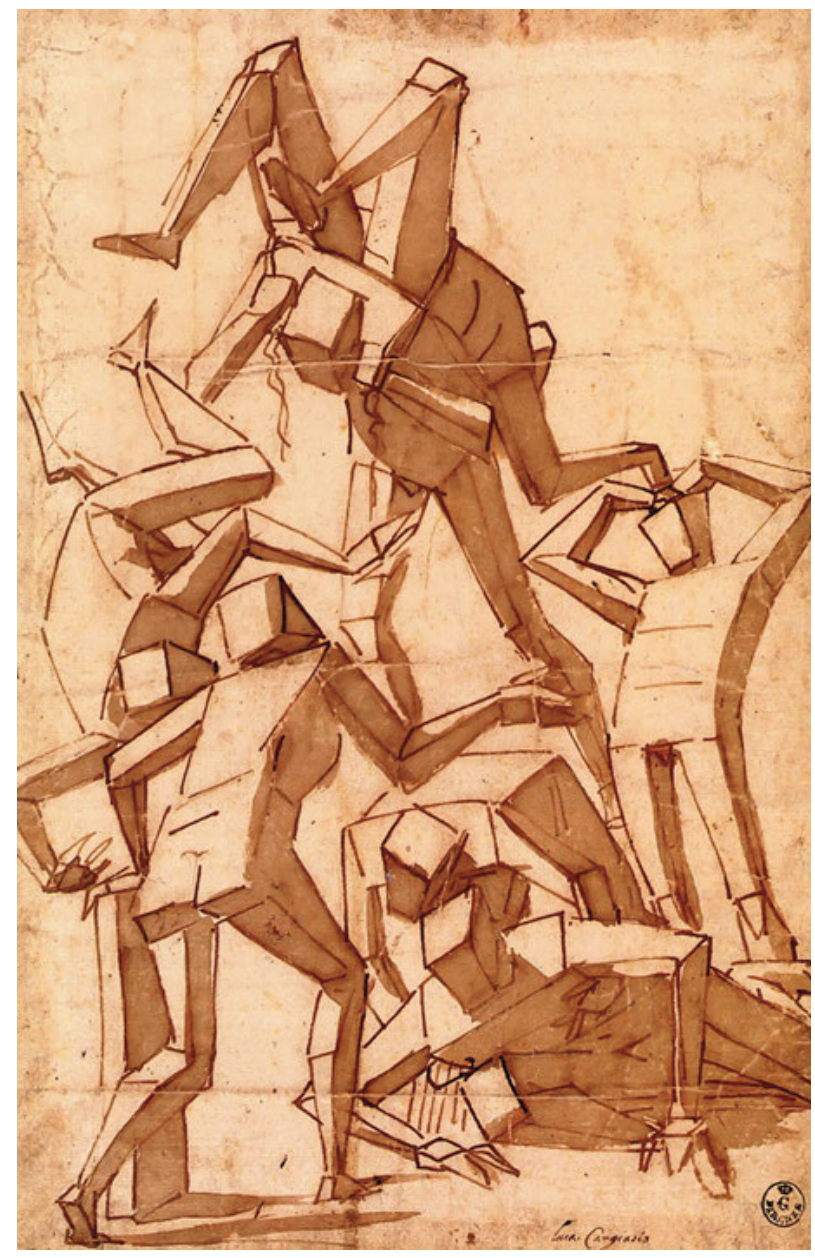

Abb. 3: Luca Cambiaso, Stürzende Körper, um 1560, Feder und Bister, $35 \times 24 \mathrm{~cm}$, Florenz, Uffizien, Inv.-Nr. 13736-F.

frühen Kubismus eines Picasso oder Braque vorauszuweisen scheinen. ${ }^{10}$ Triegels stilistisches Vorbild liegt in dieser Epoche, und hierin liegt seine Aktualität.

Dies gilt insbesondere für seinen Ariadne-Zyklus: In seiner Verwandlung der Götter erkennen Betrachtende zunächst eine Kreuzabnahme, doch da sich der Herabsteigende an einem Wollfaden orientiert, kommt das Motiv des Ariadne-Mythos hinzu. Er handelt von der Liebe der minoischen Königstochter Ariadne zu Theseus, der mithilfe eines Wollknäuels aus ihrem Labyrinth wieder herausfinden soll."

$10 \mathrm{Vgl}$. Rath 2014, $112 \mathrm{f}$.

11 Zum Ariadne-Mythos umfassend: Schlesier 2008. 


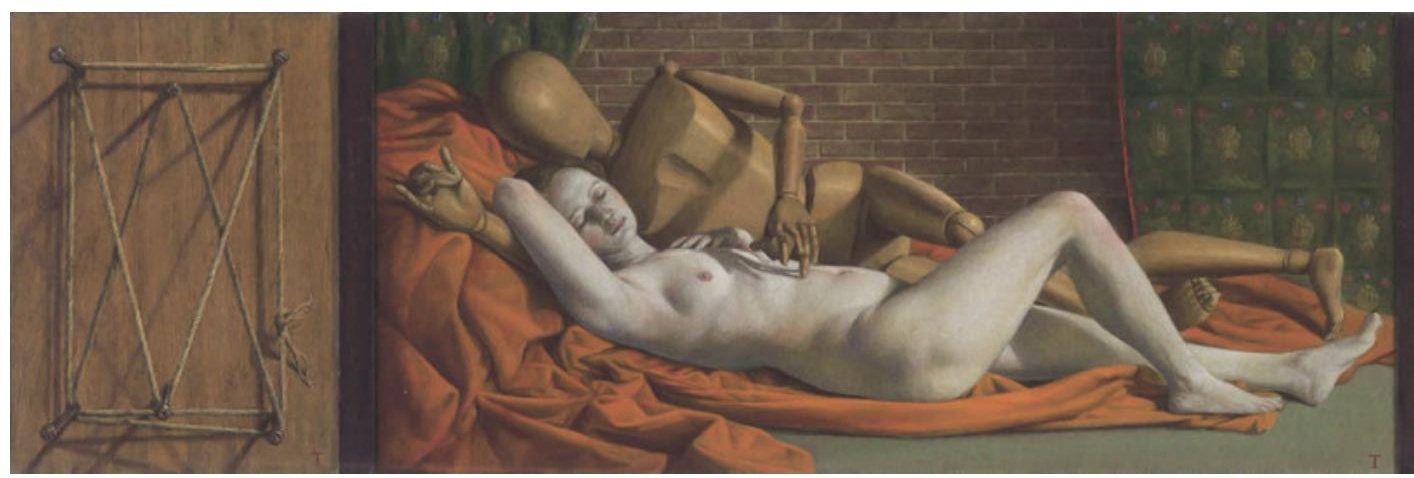

Abb. 4: Michael Triegel, Schlafende Ariadne (kleine Fassung), 2010, Mischtechnik auf MDF, 16 x $59 \mathrm{~cm}$, Rotterdam, Privatbesitz.

Die kleine Fassung der Schlafenden Ariadne zeigt das Liebespaar in seitlicher Ansicht inmitten eines Triptychons (Abb.4). Auf der Tafel links außen ist ein Holzrahmen mit dem aufgespannten Faden der Ariadne angebracht, während rechts außen das Labyrinth erscheint, aus dem Theseus mithilfe dieser Schnur den Weg ins Freie finden soll. So deutlich hier der Mythos der Ariadne thematisiert ist, so irritierend springt jene Sonderform hervor, die der Maler ihm gegeben hat. Auch bei dieser Figur handelt es sich ihrem äußeren Anschein nach nicht um ein Wesen aus Fleisch und Blut, sondern um ein Holzgebilde. Damit tritt auch hier eine jener Gliederpuppen auf, die seit der Renaissance in künstlerischen Ateliers die Möglichkeiten lebendiger Bewegung als Modellfiguren vorführen.

Noch auf einem weiteren Gemälde verwandelt Triegel den von Ariadne begehrten Helden in einen mannequino, wobei die Vertauschung der Gegensätze von tot und lebendig durch den von oben kommenden Blick auf das Paar besonders markant inszeniert ist (Abb. 5). ${ }^{12}$ Stärker noch als auf den Gemälden zuvor wirkt der Umstand wie ein befremdlicher Schock, dass der männliche Part aus einer Holzfigur besteht, die keinesfalls unbelebt ist, sondern den aktiven Part zu übernehmen scheint. Wie die Finger der linken Hand zeigen, ist sie nicht tot, sondern mit eigener Empfindsamkeit ausgestattet. Mit diesem Motiv spielt Triegel auf einen weiteren Mythos der Antike an: die Geschichte des Pygmalion. Er handelt von der Belebung der Venusstatue des Bildhauers Pygmalion zu einer veritablen Frau. Dieser Mythos wurde insbesondere zur Zeit der Aufklärung als Zeichen für die eigene Zeit verstanden, weil er als früher Beleg für die Fähigkeit galt, scheinbar tote Materie mit sensualistischer Empfindlichkeit auszustatten, welche die Grenze von organischer und anorganischer Materie überwindet. ${ }^{13}$

In seinen Ariadne-Gemälden hat Triegel die Geschlechterrollen vertauscht. Die Botschaft des Pygmalion-Mythos, dass Artefakten eine Latenz innewohnt, die aus der anorganischen in eine organische Form überspringen kann, wird bei ihm durch den mannequino verkörpert. Die Holzpuppe wird lebendig, um die Frau aus ihrem totenähnlichen Schlaf zu erwecken und durch die

12 Siehe Hüttel 2014, 187.

13 Siehe Mayer/Neumann 1997. 

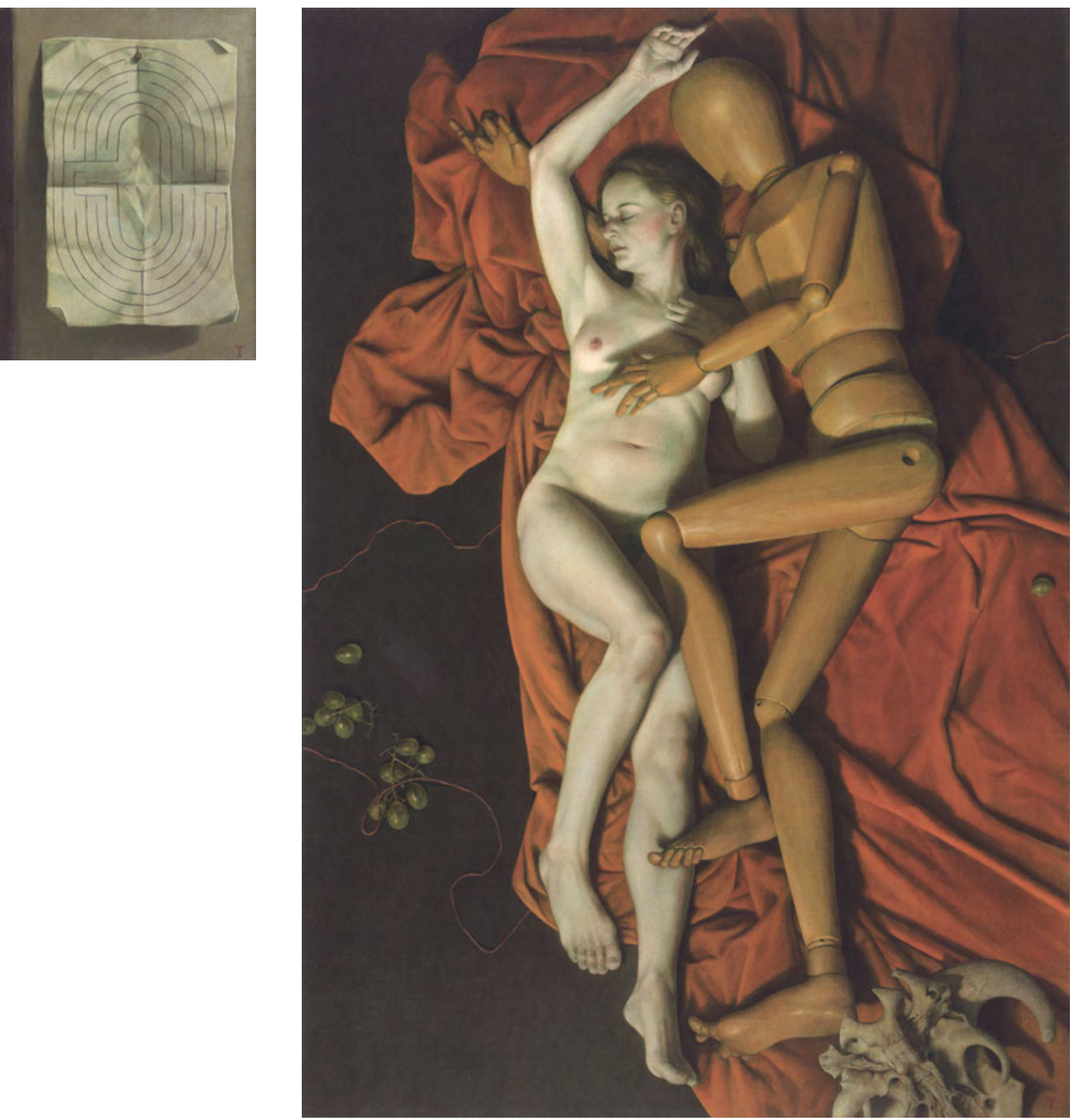

Abb.5: Michael Triegel, Schlafende Ariadne, 2010, Mischtechnik auf Leinwand, 130 x $90 \mathrm{~cm}$, Leipzig, Privatbesitz.

Fingerberührung in den Zustand wacher Sensibilität zu überführen. Es handelt sich um jene Deixis, die mit dem Fingerzeig die Schöpfung und den Akt der Belebung verbindet. Das Gemälde überkreuzt den Mythos der Ariadne mit dem des Pygmalion, um diesen zugleich umzukehren: Hier wird nicht die Frau, sondern der Mann zu jener Skulptur, die belebt auftritt. 
Zur Irritation über die Verfremdung der bekannten Ikonografien gesellt sich die Verwunderung über die Sicherheit, in der diese Transformation geschieht. Dieses inversive Moment zieht Betrachtende in das Bild hinein. Triegels Varianten des Ariadne-Mythos suggerieren das Eingeständnis, dass hier ein Bildwissen agiert, das die Freiheit des Gedankenfluges und die Kraft der Verallgemeinerung besitzt.

\section{Bild und Körper}

Mit der Verlebendigung der Holzpuppe und der Empfindlichkeit ihrer Finger thematisiert der Maler ein grundsätzliches Phänomen unserer Zeit: die Grenzaufhebung von Bild und Körper. Hierbei ist es unerheblich, ob er das von ihm dargestellte Grundproblem etwa in der Medizin vor Augen hatte, wo die Verschmelzung des menschlichen Körpers mit dem Bild seiner selbst tagtäglich geschieht. Über das Bild des Körpers wird dieser geheilt. Die gegenwärtige Medizin ist heute auch und vor allem eine Bild-Körper-Wissenschaft. Nur wer nicht versteht, dass Bilder etwas anderes sind, als sie selbst darzustellen scheinen, wird hierin von vornherein eine Erkaltung der medizinischen Profession erkennen können. Vielmehr liegt in dieser Verbilderung des Körpers eine eigene Dimension, die ebenso produktiv zu nutzen wie auch zu kritisieren ist. ${ }^{14}$ Dies mündet in eine Selbstbeobachtung, die Versicherungsgesellschaften durch sogenannte Health-Apps mit großem Einsatz vorantreiben. Der Körper wird über diese unter permanente Kontrolle gebracht, und diejenigen, die sich gewisse Formen des Unvernünftigen und Dysfunktionalen erlauben, sollen unter Zugzwang gesetzt werden. Es entstehen Bilder der Selbstbeobachtung, die den eigenen Körper in eine Assimilation mit denselben hineinziehen. Wer sich dagegen wehrt, wird in naher Zukunft vermutlich unter dem Verdacht des Ikonoklasmus mit höheren Versicherungsbeiträgen belegt werden. ${ }^{15}$

Die von Triegel thematisierte behutsame Zuwendung der Modellpuppe, die Berührung von Finger und Haut, wird aus dieser Perspektive geradezu körperlich spürbar, zumal die sehenden Fingerbewegungen im Bereich der neueren Medien zu einem eigenen Signum unserer Zeit geworden sind. In sozialer Hinsicht bedeuten sie eine instantane Verknotung des Selbst mit dem Bild des Ego oder der Gruppe. Hierzu gehört die Kokonisierung der Welt durch die Myriaden der jeden Tag von Handgerät zu Handgerät fliegenden Bilder sogenannter >Selfies<. Sie können als Teil einer Emanzipationsbewegung gedeutet werden, in der sich Menschen, die ihrer sozialen Bedeutung nach nicht dazu prädestiniert waren, bildlich in kostbare Werkzusammenhänge oder in exklusive Ausnahmesituationen hineinfotografieren, als bildliche Kommunion mit dem besonderen Kunstwerk, dem exotischen Ambiente oder dem vergänglichen Augenblick. ${ }^{16}$ Auch hierin liegt jedoch ein Angriff auf die Abstände: zwischen dem Ich und der Aura wie auch zwischen

14 Vgl. frühe Analysen wie Prassad 2005 und Badakhshi 2006 mit dem Beitrag Im Bildlabor der Neurochirurgie des vorliegenden Bandes.

15 Die europäische Versicherungsgruppe Generali bot als erster großer Versicherer seit Ende 2014 Rabatte für die elektronisch überwachte Lebensführung an, siehe Maak 2014, 11. Vgl. parallel zur Selbstverfilmung von Filmen Rothöhler 2014.

16 Siehe Bücker 2014, 11. 
dem Privaten und der Öffentlichkeit (Abb. 6). ${ }^{17}$ Im höchst ironisch inszenierten Extrem wird das Selfie zur Vision der Begrüßung einer anderen Person, womit die Selbst- zur Fremdaufnahme geworden ist, wie um die Illusion zu beseitigen, dass hier eine Ich-Erzählung am Werk ist. ${ }^{18}$

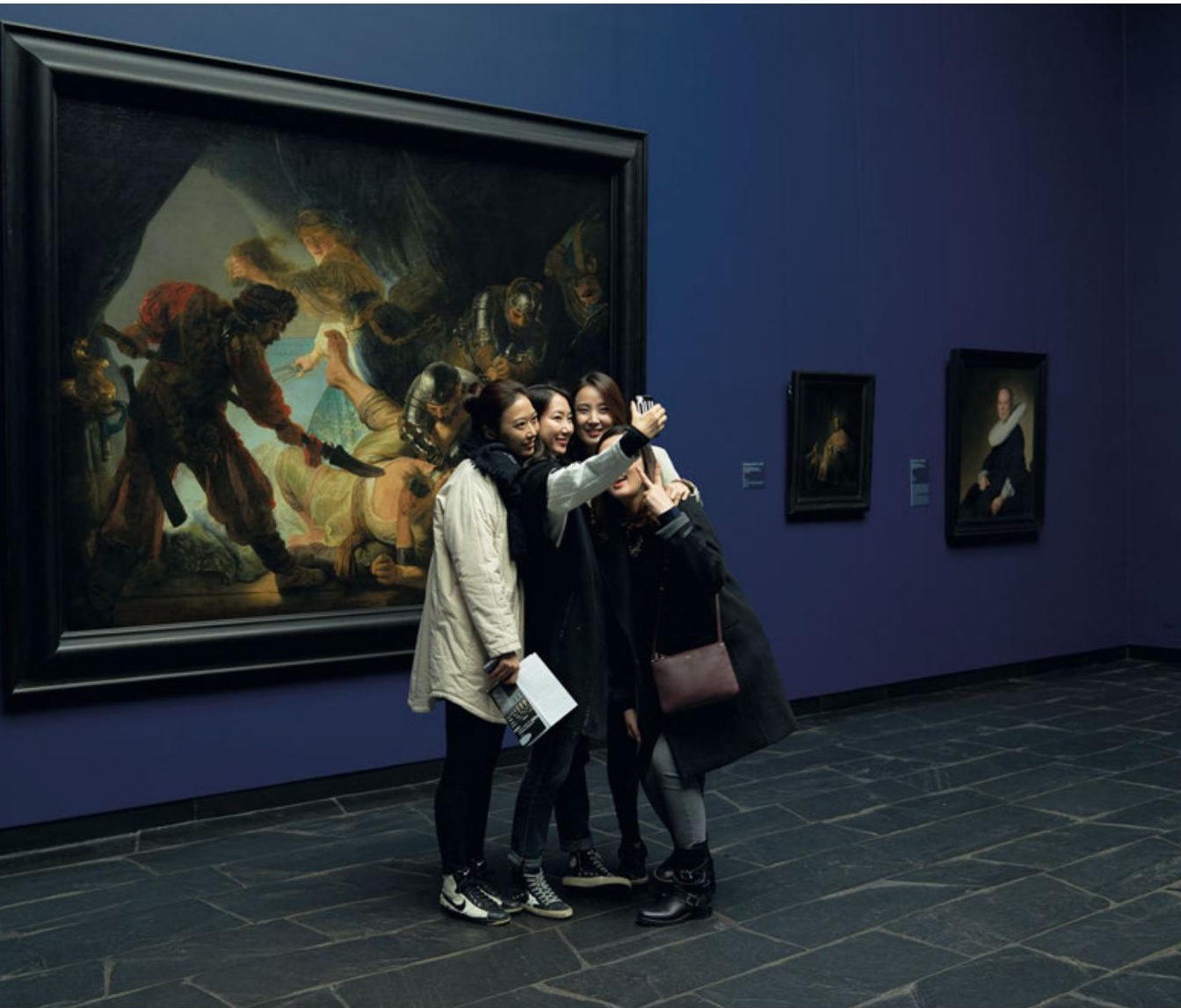

Abb. 6: Selfieaufnahme vor Rembrandts Die Blendung Simsons im Städel Museum, Frankfurt a. M.

17 Siehe Hierholzer 2015, 8.

18 Zu diesem Beispiel siehe das Video zum selfie arm auf der Homepage $h$ ttp://Gabc.com/business/would-you-buy-a-selfiearm-to-take-pictures/688633/ (zuletzt aufgerufen: 8. Juli 2015). 


\section{Natur- und Kunstform}

Als Emblem für den Exzellenzcluster Bild Wissen Gestaltung wurde eines der um 1560 geschaffenen stereometrischen Gebilde des Manieristen Wenzel Jamnitzer gewählt (Abb. 7). Es handelt sich um eine jener Spiralenformen, die auch in gedrechselter Form die Gegensätze von Verschalung und Transparenz, Innen und Außen symbolisiert haben (Abb. 8).

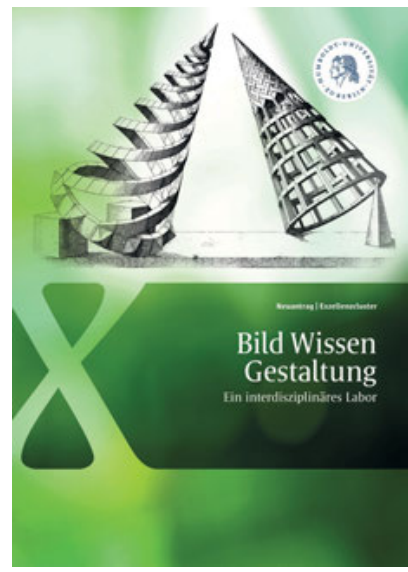

Abb. 7: Titelbild des Antrages auf Einrichtung des Exzellenzclusters Bild Wissen Gestaltung, August 2011.

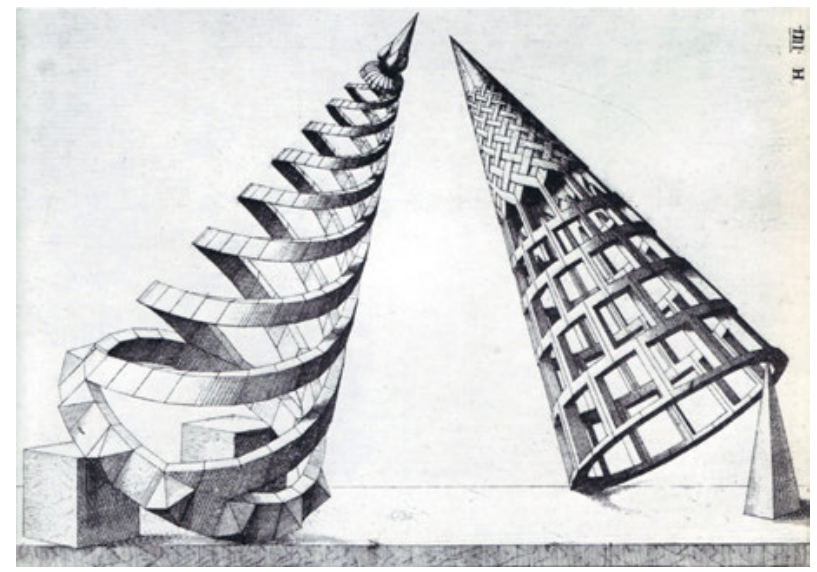

Abb. 8: Jost Amman, Perspektivdarstellung zweier kegelförmiger Körper, 1568, Kupferstich.

Als im Jahr 1957 Gustav René Hockes Publikation Die Welt als Labyrinth erschien, hatte sie eine ähnliche Figur aus Johannes Lenckers Perspectiva literaria von 1567 auf dem Titel. Er zeigte die geometrische Konstruktion einer Nautilusschnecke, die in der zeitgenössischen Kunst um 1600 zu einem Hauptmotiv der bildnerischen Produktion wurde, weil ihre Form auf die strukturelle Übergangsfähigkeit sowie die Austauschbarkeit von Kunst- und Naturform hinwies (Abb. 9). ${ }^{19}$ In der Natur selbst schien ein Prinzip angelegt, das die Mathematik mühsam erschlossen und die Kunst geradezu lustvoll nachgeahmt hatte.

Wie sehr dieses Prinzip zu einem gegenwärtigen Leitmotiv geworden ist, vermag ein winziges, etwa zwei Zentimeter hohes Gebilde zu verdeutlichen. Aus einer bestimmten Perspektive ist die Gehäuseoberfläche geschlossen, sodass der Eindruck einer zylindrischen Form entsteht. Leicht gedreht, wird der Körper aber zu einem transparenten Gebilde (Abb. 10): Mit der Erscheinungsweise hat er sein Wesen verändert. Ein einfacher Perspektivwechsel macht aus einer offenen eine geschlossene Form: Und genau hierin ist diese großartige kleine Skulptur 
ein Emblem unserer Zeit. ${ }^{20}$ Es handelt sich um das nachgebildete Innere eines Knochens, das als Modellfigur des Max-Planck-Instituts für Kolloid- und Grenzflächenforschung geschaffen wurde. ${ }^{21}$ Es lässt die Grenze von Kunst- und Naturform verschwimmen und formuliert damit eine Grundüberzeugung des Manierismus.

Wohl niemand hat die Überwindung dieser scheinbar so scharf gezogenen Grenze in eine treffendere Formel gebracht als der Dichter Torquato Tasso. Seinem Diktum zufolge ist es die

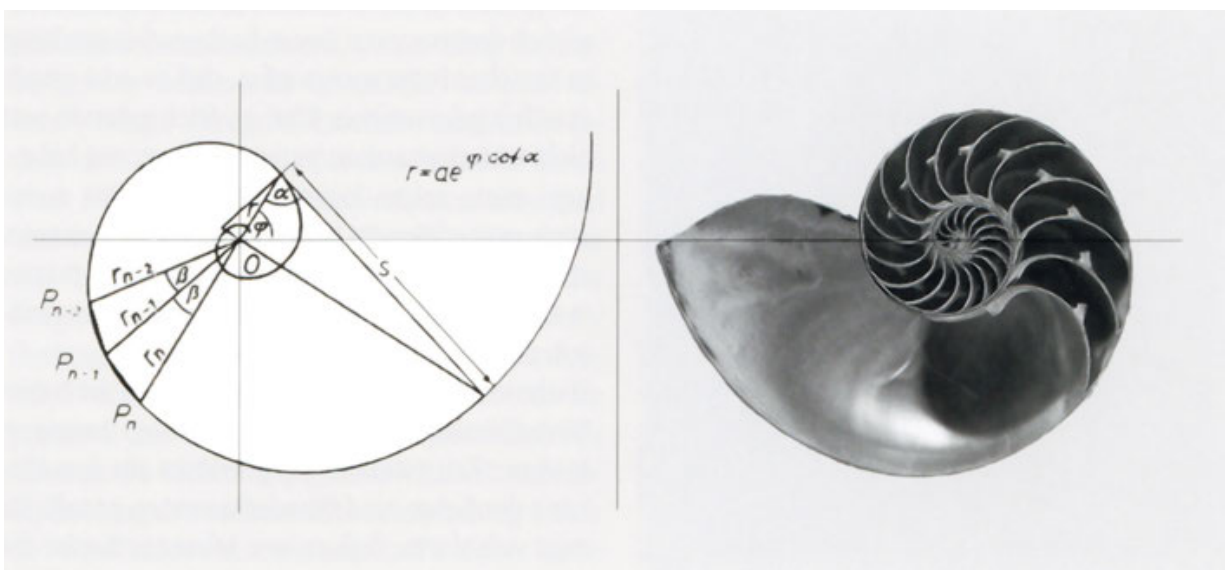

Abb. 9: »Die logarithmische Spirale in ihrer mathematischen Konstruktion und natürlichen Nautilusgestalt «.

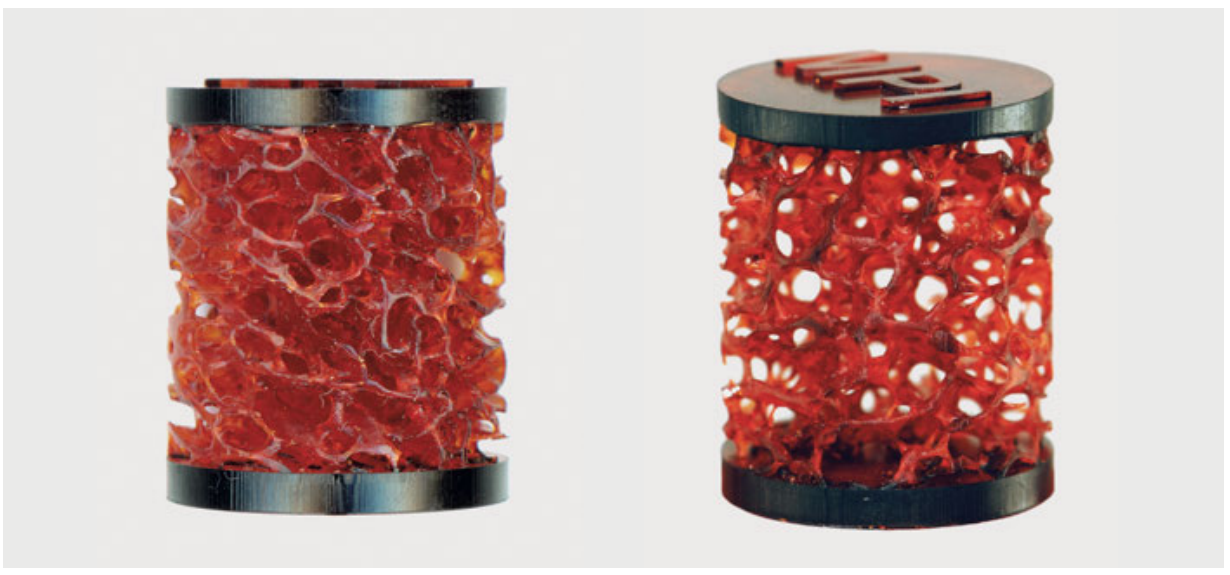

Abb. 10: 3-D-Modell des Ausschnitts eines Wirbelknochens, ca. 2013, Kunststoff, Max-Planck-Instituts für Kolloid- und Grenzflächenforschung, Berlin.

20 Zu den Begriffen offene und geschlossene Form: Wölfflin 1991, 147-180.

21 Mit freundl. Genehmigung von Peter Fratzel. 
Natur selbst, welche in einem die Maßstäbe verrückenden Spiel eine Kunst nachahmt, die sich ihrerseits als Imitatorin der Natur ausgegeben hat. »Die Kunst sei nur Natur, die wie im Spiel / Zu ihrer eigenen Lust, das Nachbild nachahmt. «22 Dies ist das Urprinzip des Manierismus, und mit ihm könnte das Gebilde des Max-Planck-Instituts nicht besser beschrieben sein.

Im globalen Maßstab vollzog sich dies in der Formgestaltung der Erde, die mit dem Begriff des Anthropozäns belegt worden ist. Im Jahr 2000 haben der niederländische Nobelpreisträger Paul Crutzen und Eugene F. Stoermer den Begriff geprägt, um anzuzeigen, dass die Erde in geologischer Hinsicht zu einem Produkt des Menschen geworden sei. In den oberen Gesteinsstrukturen hätten sich die Spuren der Industrialisierung derart flächendeckend eingetragen, dass von einer neuen, humanoid geprägten geologischen Epoche der Erdkugel auszugehen sei. ${ }^{23}$ Damit aber ist die Formung der Erde durch den Menschen so weit vorangeschritten, dass Gestaltung bildproduktiv wirksam wird. Die gesamte Erde ist solcherart zu einem Bild geworden, wie dies Matthäus Merian imaginiert hat (Abb. 11), ${ }^{24}$ sodass jede Darstellung derselben nicht etwa eine an sich vorzustellende Natur, sondern die Vermischung von ungeformter Materie und Bildwerdung bedeutet.

Abb. 11: Matthäus

Merian d. Ä.,

Nutrix Terra, 1617 , Kupferstich.

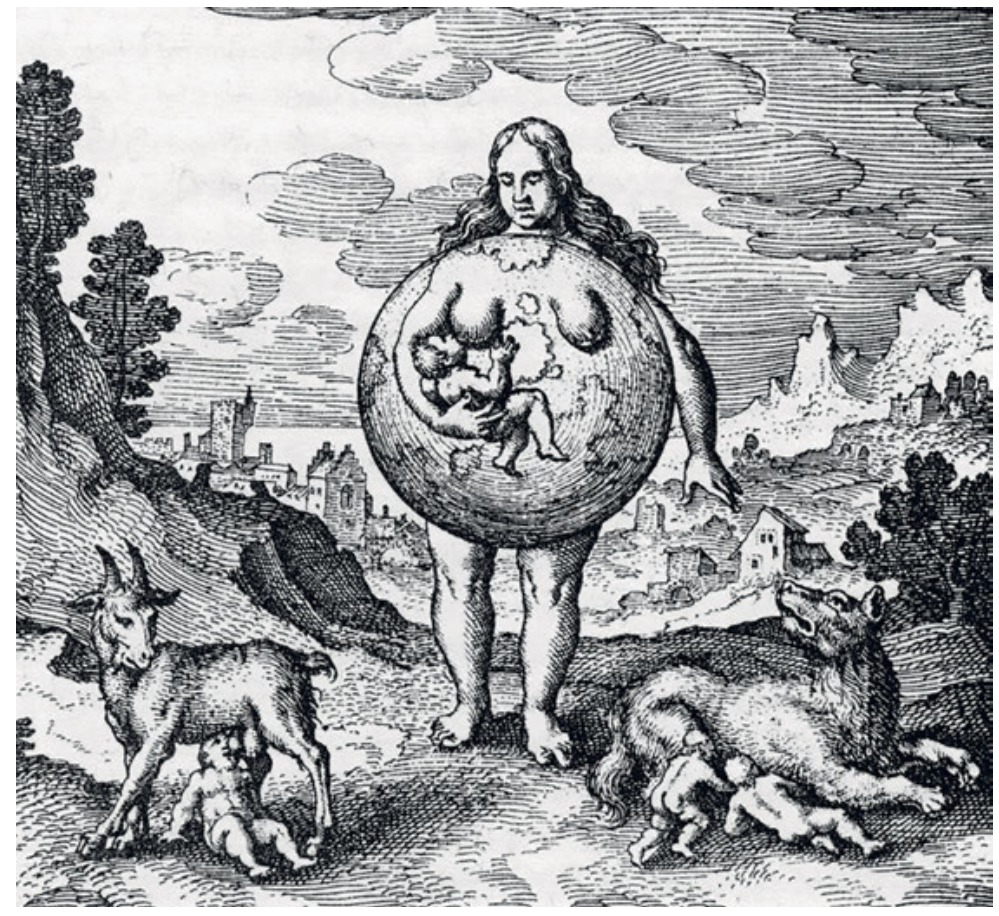

$22 \gg$ Di natura arte par, che per diletto / l'imitatrice sua scherzando imiti«, Tasso 1575, 387, 16, 10, 3f., zit. aus der von Emil Staiger übers. Ausg. 1978, 536.

23 Siehe Crutzen/Stoermer 2000, 17-18. Vgl. Crutzen 2002, 23; ders. 2011. Vgl. außerdem den Beitrag Wissenschaftliche Sachcomics im vorliegenden Band.

24 Siehe Bredekamp 2011. 


\section{Innen und Außen}

Im Mikrobereich ist es die Welt des Mikroskopes, die zu einer Grenzaufhebung von Kunst und Organik führt, und dies mit nur ahnbaren naturphilosophischen Konsequenzen. Diese Entwicklung hängt unter anderem mit Quallen zusammen. Als Auslöser kann die Aequorea victoria gesehen werden, eine im Nordpazifik beheimatete Qualle. Sie ist berühmt dafür, dass eines ihrer Proteine bei Berührung mit Calciumionen eine Bioluminiszenz erzeugt, die sich auch in anderen Organismen provozieren lässt. Dies macht ihre ungeheure Bedeutung aus. ${ }^{25}$ Um die Bilderwartung der naturwissenschaftlichen Analyse erfüllen zu können, wird für die Stimulated Emission Depletion dem zu untersuchenden lebenden Stoff ein fluoreszenter Farbstoff einverleibt. Er übernimmt die Funktion eines Leuchtfeuers, das unterhalb der Wellenlänge des Lichts agiert und dennoch sichtbar wird. Die Natur wird verändert, um bildpotent werden zu können. ${ }^{26}$

Eine Variante dieses Verfahrens stellt die Optogenetik dar, bei der über einen Retrovirus Abschnitte von Algen- und Quallengenen, ebenfalls fluoriszierend, in Nervenzellen eingeschleust werden. Bei Befeuerung mit ultraviolettem Licht reagieren diese Zellen, sodass eine bislang unbekannte Kartierung der Nervenbahnen möglich wird. Über die lichterzeugte Aktivierung von Hirnzellen bei Mäusen wird deren Gedächtnisleistung zum kolorierten Bild (Abb. 12). ${ }^{27}$

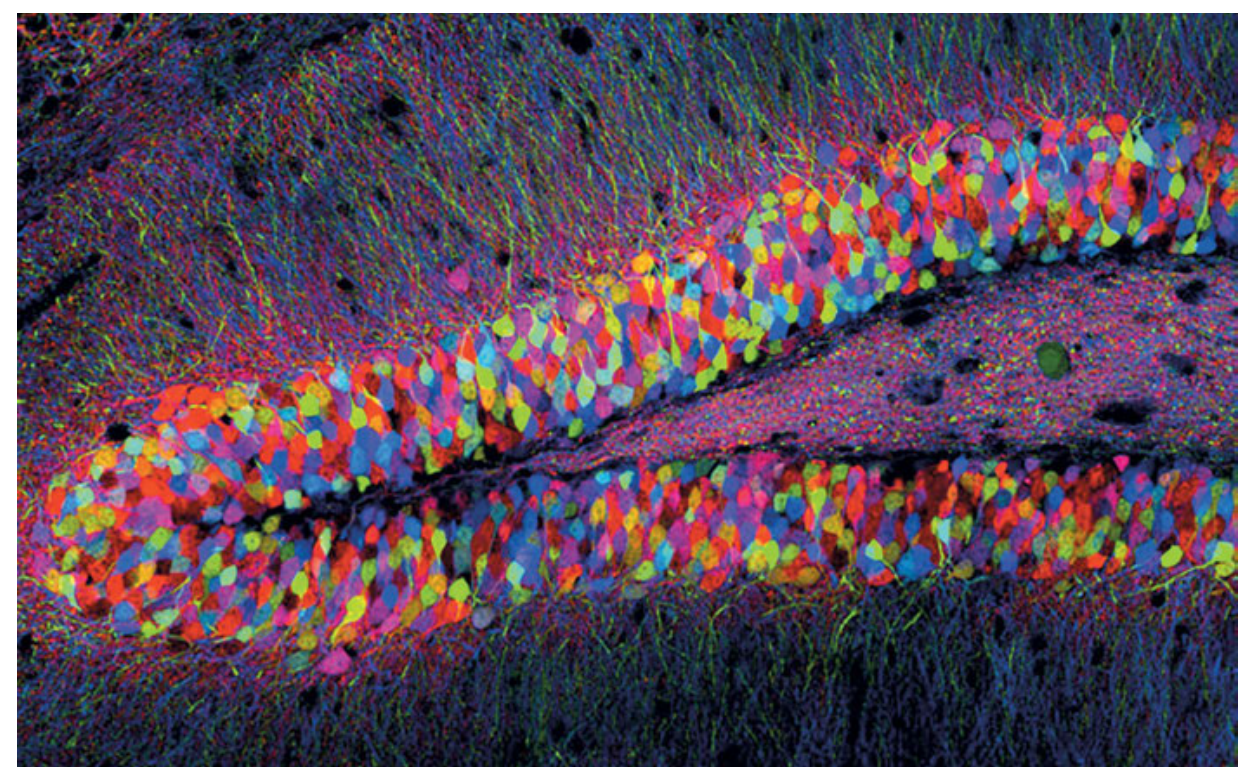

Abb. 12: > Mosaikbild < des Farbausdrucks fluoreszierender Proteine im Mäusehirn.

25 Vgl. ausführlicher Bredekamp 2011 .

26 Siehe Abbot 2009, 638, sowie zu den bildtheoretischen Konsequenzen Schwamborn 2006. Zur Gesamtentwicklung

Pieribone/Gruber 2005. Einen Durchbruch markierte der Artikel von Hell/Rittweger 2009.

27 Siehe Weissmann et al. 2011. 
Aber nicht hierin liegt das Ungeheure des Vorgangs, sondern in der Fähigkeit, die Wege der Bilderzeugung in Kanäle der Steuerung umzukehren. Über die Tunnel, welche die sogenannten Kanalrhodopsine in den angereicherten Zellen erzeugen, sind diese durch niederwelliges Licht ein- und auszuschalten. Die Bildanalyse wird zum Medium der in Lichtgeschwingkeit durchzuführenden Befehlsgebung. ${ }^{28}$

Um Bilder einer Naturform zu generieren, wird zunächst ein Zwitter hergestellt, der in der Lage ist, diese Bilder zu erzeugen. Auf diese Weise entstehen auf Zeit neue Organformen, deren Existenzberechtigung in ihrer bildaktiven Potenz liegt. Seit den ersten Publikationen zeigt sich immer wieder das Faszinosum über die Möglichkeiten einer veränderten Natur, die eigene Farben im Sinne eines Brainbow getauften Ausdruckstheaters erzeugt ${ }^{29}$ und einem aus dem Innersten kommenden Fernsehprogramm exaltierter, oft überraschend ausfallender Farben zu gleichen scheint. ${ }^{30}$ Hier deutet sich ein symbolischer Begriff der Natur an, der zugleich sie selbst und die ihr zugewachsene Bilderwartungsorganik umfasst: als neomanieristische Gebilde par excellence.

\section{Möglichkeiten der Theorie}

All diese Phänomene haben einen mitreißenden und einen kritischen Zug, und umso drängender sollte das Gebot sein, sie in eine Theorie zu bringen. Indem der Manierismus die Grenzziehung von natürlich und künstlich, tot und lebendig sowie normativ und subversiv systematisch infrage gestellt hat, erscheint er als Spiegelbild unserer eigenen Zeit. Der Neomanierismus ist als gesamtkulturelles Phänomen zu beschreiben, in all seiner Produktivität wie auch seinen problematischen Zügen. Es handelt sich um das Phänomen, dass die Trennungen, welche zwischen Bild und Natur, Bild und Körper bestanden haben, in immer weiteren Bereichen nicht nur symbolisch, sondern auch materiell aufgehoben werden.

Im Sinne einer angemessenen Theorie unserer Zeit muss es um eine harmonieresistente Bindung von Gegensätzen gehen, um eine Reflexion der Aufhebung von Grenzlinien: zwischen Bild und Körper, zwischen außen und innen sowie zwischen privat und öffentlich. Dieser Prozess ist vielfach beobachtet, aber nicht ansatzweise in eine Theorie gebracht worden, welche der Tiefe des Problems angemessen wäre.

Der gegenwärtige Neomanierismus definiert sich nicht allein in der Kunst, aber es scheint, als sei diese nach wie vor im Besitz eines eigenen Bilderwissens. Die Kunst ist Teil eines Phänomens, das sie transzendiert. Die Grenzaufhebung von Bild und Körper, von artifiziell und lebendig, von privat und offenkundig sowie von visuell und haptisch: All dies ist in den erörterten Gemälden aufgenommen. Hierin sind sie theoriebildend, ohne begrifflich auftreten zu müssen. 


\section{Literatur}

Abbott, Alison (2007): Colours Light Up Brain Structure. In: Nature News, 31 October. Online: http://www.nature. $\mathrm{com} /$ news $/ 2007 / 071031 /$ full/news.2007.209.html (last access: 10 June 2015).

Abbot, Alison (2009): Microscopic Marvels. The Glorious Resolution. In: Nature, vol.459, no.7247, pp. 638-639. Online: http://www.nature.com/news/2009/090603/ full/459638a.html (last access: 10 June 2015).

Badakhshi, Harun R. (2006): Körper in/aus Zahlen. Digitale Bildgebung in der Medizin. In: Hinterwaldner, Inge/Buschaus, Markus (Hg.): The Picture's Image. Wissenschaftliche Visualisierung als Komposit. Paderborn/ München: Fink, S. 199-205.

Battisti, Eugenio (1962): L'Antirinascimento. Milano: Garzanti.

Bredekamp, Horst (2000): Der Manierismus. Zur Problematik einer kunsthistorischen Erfindung. In: Manier und Manierismus (Untersuchungen zur deutschen Literaturgeschichte, 106). Hg. v. Braungart, Wolfgang. Tübingen: Niemeyer, S. 109-129.

Bredekamp, Horst (2011): Blue Marble. Der Blaue Planet. In: Atlas der Weltbilder. Hg. v. Markschies, Christoph u. a. Berlin: Akademie Verlag, S. 366-375

Bredekamp, Horst (2011a): In der Tiefe die Künstlichkeit. Das Prinzip der bildaktiven Disjunktion. In: ders./Krois, John M. (Hg.): Sehen und Handeln (Actus et Imago. Berliner Schriften für Bildaktforschung und Verkörperungsphilosophie, 1). Berlin: Akademie Verlag, S. 206-224.

Bücker, Teresa (2014): Das nackte Selbst. Selfies sind ein Akt der Emanzipation. In: DIE ZEIT, Nr. 42, 9. Oktober.

Crutzen, Paul J./Stoermer, Eugene F. (2000): The »Anthropocene«. In: Global Change Newsletter, no.41, pp. 17-18.

Crutzen, Paul J. (2002): Geology of Mankind. In: Nature, vol. 415 , no. 6867 , p. 23 .

Crutzen, Paul J. (2011): Die Geologie der Menschheit. In: ders. u. a.: Das Raumschiff Erde hat keinen Notausgang. Energie und Politik im Anthropozän. Berlin: Suhrkamp, S. 7-10.

Curtius, Ernst Robert (1948): Europäische Literatur und Lateinisches Mittelalter. Bern: Francke.
Frieß, Peter (1993): Kunst und Maschine. 500 Jahre Maschinenlinien in Bild und Skulptur. München: Deutscher Kunstverlag.

Gastel, Joris van/Hadjinicolaou, Yannis/Rath, Markus (Hg.) (2014): Paragone als Mitstreit. Berlin: Akademie Verlag.

Hegemann, Peter/Sigrist, Stephan (Hg.) (2013): Optogenetics. Berlin: De Gruyter.

Hell, Stefan W./Rittweger, Eva (2009): Light From the Dark. In: Nature, vol.461, no.7267, pp.1069-1070.

Hierholzer, Michael (2015): Museumsalltag: Selfie vor Gewaltdarstellung. In: Frankfurter Allgemeine Sonntagszeitung, Nr. 11, 15. März.

Hocke, Gustav René (1957): Die Welt als Labyrinth. Manier und Manie in der europäischen Kunst. Reinbek bei Hamburg: Reclam.

Hüttel, Richard (Hg.) (2014): Werner Tübke. Michael Triegel. Zwei Meister aus Leipzig. Ausst.-Kat. Kunsthalle Rostock/Kunsthalle Jesuitenkirche, Aschaffenburg, München: Hirmer.

Huss, Bernhard/Wehr, Christian (Hg.) (2014): Manierismus. Interdisziplinäre Studien zu einem ästhetischen Stiltyp zwischen formalem Experiment und historischer Signifikanz. Heidelberg: Universitätsverlag Winter.

Jamnitzer, Wenzel (1568): Perspectiva Corporum Regularium. Nachdr. d. Nürnberger Ausg. Graz: Akadem. Druck-u. Verlagsanst. 1973.

Leeflang, Huig et al. (2003): Hendrick Goltzius (15581617). Drawings, Prints and Paintings. Exh. cat. Rijksmuseum, Amsterdam et al. Zwolle: Waanders.

Livet, Jean et al. (2007): Transgenetic Strategies for Combinatorial Expression of Fluorescent Proteins in the Nervous System. In: Nature, vol.450, no. 7166, pp. 56-62.

Maak, Niklas (2014): Die Veröffentlichung unserer Körper. In: Frankfurter Allgemeine Zeitung, Nr. 276, 27. November.

Maier, Michael (1618): Atalanta Fugiens hoc est emblemata nova de secretis naturae chymica. Hg. v. Wüthrich, Lucas H. Faksimile-Nachdr. der Oppenheimer Originalausg. Kassel: Bärenreither 1964. 
Mayer, Mathias/Neumann, Gerhard (Hg.) (1997): Pygmalion. Die Geschichte des Mythos in der abendländischen Kultur. Freiburg i. Br.: Rombach.

Mette, Hans-Ulrich (1995): Der Nautiluspokal. Wie Kunst und Natur miteinander spielen. München/Berlin: Klinkhardt \& Biermann.

Mette, Hans-Ulrich: Die Nautilus-Abteilung. In: Karin Annette Möller (Hg.) (2013): Schimmern in der Tiefe. Muscheln, Perlen, Nautilus. Ausst.-Kat. Staatliches Museum Schwerin. Petersberg: Michael Imhof Verlag, S. $146-178$.

Milton, Joseph (2011): Fly Brain Structure Illuminated. In: Nature News, 6 February. Online: http://www.nature. com/news/2011/110206/full/news.2011.73.html (last access: 10 June 2015)

Pieribone, Vincent/Gruber, David F. (2005): A Glow in the Dark. The Revolutionary Science of Biofluorescene. Cambridge, MA: Harvard University Press.

Prassad, Amit (2005): Making Images/Making Bodies. Visibilizing and Disciplining through Magnetic Resonance Imaging (MRI). In: Science, Technology, and Human Values, vol. 30, no. 2, pp. $451-467$.

Rath, Markus (2014): Manipulationen. Modellform, Bildrelief und kubische Denkfigur als Moment des Mitstreits. In: Gastel/Hadjinicolaou/Rath 2014, S. 99-118.

Rothöhler, Simon (2014): Desktop-Arbeit. Filmkolumne. In: Merkur, Jg. 68, Bd. 784, Nr. 9, S. 812-816.
Schlesier, Renate (2008): Ariadne. In: Cancik, Hubert/ Landfester, Manfred/Schneider, Helmuth (Hg): Der Neue Pauly. Supplemente, Bd. 5: Mythenrezeption. Die antike Mythologie in Literatur, Musik und Kunst von den Anfängen bis zur Gegenwart. Hg. v. Moog-Grünewald, Maria. Stuttgart/Weimar: Metzler, S.140-150.

Schwamborn, Klaus (2006): Farben als Proteine: Wie aus Bildern neue Organismen werden. In: Bredekamp, Horst/ Bruhn, Matthias/Werner, Gabriele (Hg.): Bildwelten des Wissens. Kunsthistorisches Jahrbuch für Bildkritik, Bd.4.1: Farbstrategien. Berlin: Akademie Verlag, S. 17-24.

Staiger, Emil (Hg.) (1978): Torquato Tasso. Werke und Briefe. München: Winkler.

Tasso, Torquato (1575): La Gerusalemme liberata. Florenz: Salini 1976.

Torriti, Piero (1969): Luca Cambiaso. Disegni. Genua: Sagep.

Weissmann, Tamily A. et al. (2011): Generating and Imaging Multicolor Brainbow Mice. In: Cold Spring Harb Protoc, pp. 763-769. Online: http://cshprotocols. cshlp.org/content/2011/7/pdb.top114.full.pdf + html (last access: 10 June 2015).

Wölfflin, Heinrich (1991): Kunstgeschichtliche Grundbegriffe. Das Problem der Stilentwicklung in der Neuern Kunst. 18. Aufl. Basel: Schwabe. 


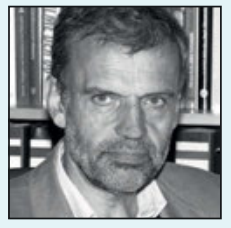

horst.bredekamp@culture.hu-berlin.de

Principal Investigator, Sprecher

Basisprojekt: Bildakt

Disziplin: Kunst- und Bildgeschichte

Horst Bredekamp ist Professor für Kunstgeschichte an der Humboldt-Universität zu Berlin und seit 2012 Sprecher des Exzellenzclusters Bild Wissen Gestaltung. 2000 gründete er das Projekt Das Technische Bild am Hermann von Helmholtz-Zentrum für Kulturtechnik. Seit 2003 ist Bredekamp Permanent Fellow des Wissenschaftskollegs und leitet seit 2008 zudem die neu eingerichtete DFG-Kolleg-Forschergruppe Bildakt und Verkörperung, die als Basisprojekt Bildakt im Cluster aktuell weitergeführt wird. 


\section{Im Bildlabor der Neurochirurgie}

\section{Ein interdisziplinärer bildgeführter Fallbericht}

Robert E., 33-jähriger Patient, wurde vor zwei Wochen nach einem akuten Krampfanfall in der Rettungsstelle vorstellig. Er berichtete von neu aufgetretenen Kribbelparästhesien und einem Sensibilitätsverlust im rechten Bein. Dazu eine transiente Parese im rechten Arm, die sich innerhalb weniger Stunden vollständig zurückbildete. Bei der körperlichen Untersuchung fiel zusätzlich eine leichtgradige Parese der Fußmuskulatur rechts auf. Sonstige neurologische Untersuchung unauffällig. Patient normoton. Medikamentenanamnese leer. Zum Blutungsausschluss wurde ein natives CT gemacht: dringender Verdacht aufeine intrakranielle Raumforderung, keine Blutung. Zur weiteren Abklärung erfolgte ein MRT: Dort zeigt sich eine Kontrastmittel aufnehmende Läsion im Bereich des linken primär motorischen Kortex von etwa 2,5 $\times 2 \mathrm{~cm}$ sowie eine relativ ausgedehnte subkortikale Infiltrationszone. Keine raumfordernde Wirkung. Am ehesten vereinbar mit einem astrozytären Tumor.

Im medizinischen Alltag gibt es oftmals wenig Raum für eine tiefgreifende Bildkritik, da die Antworten, die Bilder geben sollen, häufig vorstrukturiert sind. Aus einer geistes- und sozialwissenschaftlichen Perspektive hingegen stand die klinische Handlungsmacht medizinischer Bilder lange nicht im Fokus der Analysen, sondern deren ästhetische Charakteristika sowie die sozialen Kontexte ihrer Herstellung und Zirkulation. ${ }^{1}$ Bildgebende Verfahren gestalten zunehmend die Therapie und fungieren dort als zentrale Schnittstelle zwischen Arzt_Ärztin und Patient_in. Bildkompetent müssen etwa Neurochirurg_innen folglich nicht mehr nur als Diagnostizierende und während der Planungsphase sein, vielmehr müssen sie ebenso über ein anwendungsorientiertes Bildwissen bei therapeutischen Interventionen verfügen.

1 Vgl. etwa Alač 2011; Burri 2008; Joyce 2008. 
Viele Patient_innen werden im Verlauf ihrer Behandlung von einer komplexen Bildkette begleitet, deren einzelne Elemente nicht nur in einer bestimmten zeitlichen Abfolge entstehen, sondern auch technisch aufeinander aufbauen und vielfach miteinander fusioniert werden. Eine typische Fallgeschichte in der Neuroonkologie beginnt mit einer unspezifischen, möglichst viele Differenzialdiagnosen abdeckenden Überblicks- bzw. Ausschlussdiagnostik: Der behandelnde Arzt oder die behandelnde Ärztin möchte akute, potenziell lebensbedrohliche Zustände (wie etwa eine intrazerebrale Blutung oder eine Infektion der Hirnhäute) ausschließen und bei Verdacht auf eine Tumorerkrankung über die entscheidenden Informationen verfügen (Größe, Lage). Das > klassische< erste Bild einer solchen Kette liefert häufig die Computertomografie (CT), die ubiquitär verfügbar und mit einem geringen Zeitaufwand verbunden ist. Ihre Nachteile liegen primär in der Strahlenbelastung der Patient_innen sowie in der niedrigen Auflösung der Weichteilkontraste. In vielen neurochirurgischen Fällen unverzichtbar ist daher ein anderes Bildgebungsverfahren, ${ }^{2}$ das Hirnstrukturen mit hoher Kontrastauflösung darstellen kann.

Moderne radiologische Bildgebungsverfahren wie die Magnetresonanztomografie (MRT) erzeugen in komplexen Prozessen Visualisierungen des inneren Patient_innenkörpers. Diese sind grundlegend für diagnostische Entscheidungen und therapeutische Planungen, da sie präoperativ häufig den alleinigen Zugang zu einer fraglichen Pathologie bieten. ${ }^{3}$

Detailreiche MRT-Visualisierungen sind vor allem in der neurochirurgischen Therapie von besonderer Bedeutung (Abb. 1). Die hier gezeigten Magnetresonanztomografien stehen exemplarisch für eine ganze Serie von Schnittbildrekonstruktionen in den drei Körperebenen axial, sagittal und koronar. Eine solche multiplanare Rekonstruktion wird computergrafisch aus Messwerten rekonstruiert, die mithilfe eines MRT-Scanners gewonnen wurden. Neurochirurg_innen scrollen mittels Softwareanwendungen durch verschiedene Schnittbildserien, um die Lage und Größe eines Tumors abschätzen zu können.

Das diagnostische Potenzial von MRT-Visualisierungen ergibt sich bei genauerer Betrachtung nicht allein aus ihrer bildhaften Darstellung, sondern auch aus den digitalen Berechnungs- und Prozessierungsschritten, die auf verschiedenen Ebenen Relationen zwischen Patient_in, Bild und diagnostisch-chirurgischem Blick konstituieren. Die Frage nach dem diagnostischen Wert graustufiger Schnittbilder in Zeiten mehrdimensionaler und farbiger Modellierungen muss somit nicht nur an die Oberfläche des Bildes gerichtet werden.

Der grundlegende Visualisierungsprozess der Magnetresonanztomografie ordnet Messwerten ästhetische Merkmale in Form von Graustufen zu. So verweist die bildhafte Darstellung immer auch auf eine physikalisch-indexikalische Relation zum Patient_innenkörper. Eine solche Zuordnung von Mess- zu Grauwerten basiert neben technischen auch auf ästhetischen Konventionen und Traditionen. Die Möglichkeit, die Messwerte als Zahlenmatrix auszudrucken und daraus eine diagnostische Aussage zu treffen, wurde bereits in der klinischen Frühphase der Magnet-

2 Vgl. ausführlicher Bruzzone et al. 2012.

3 Vgl. Beaulieu 2002. 


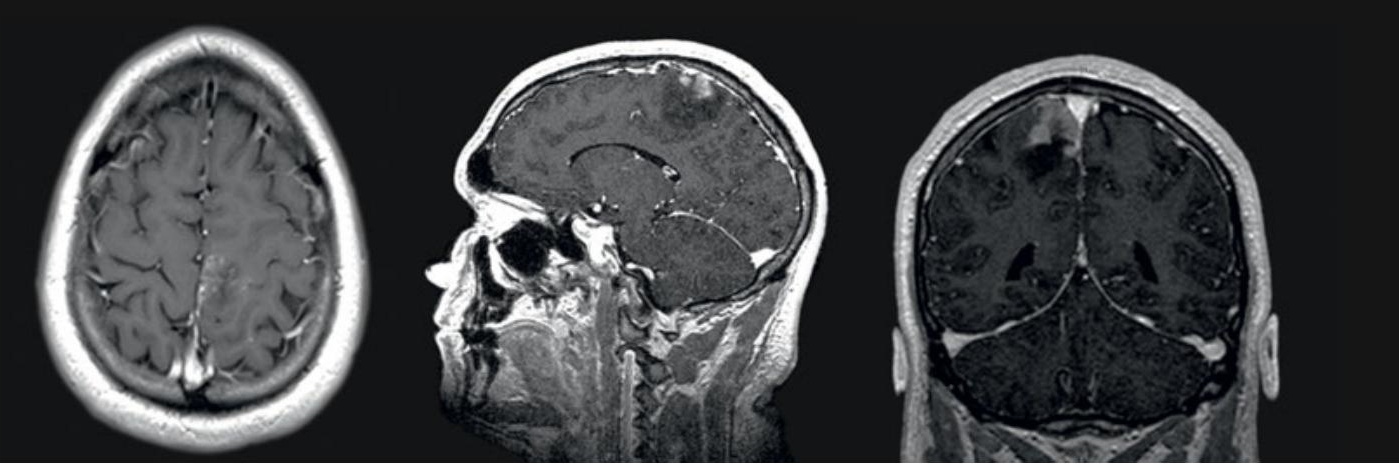

Abb. 1: Präoperatives MRT-Bild des Gehirns in drei Ebenen (T1 nach Kontrastmittelgabe). Zum besseren Verständnis der Bildkette wurden die axiale und koronare Darstellung gespiegelt (im Bild links = am Patienten links).

resonanztomografie verworfen. ${ }^{4}$ Zu dominant waren kollektive Wahrnehmungsstrategien einer röntgenologischen Bildästhetik bereits in klinischen Kontexten verankert.

Die Graustufenästhetik konstituiert epistemische Potenziale, die über das Bild hinaus auf gestalttheoretische Perspektiven und physiologische Aspekte der Bildwahrnehmung verweisen. ${ }^{5}$ Insbesondere Radiolog_innen sind geübte >Grauseher<, da bereits ihre Ausbildung an graustufigen Röntgenbildern, CT- und MRT-Visualisierungen ausgerichtet ist. ${ }^{6}$ Daher ist es nicht nur eine Frage der individuellen Präferenz, ob MRT-Daten als mehrdimensionale, farbige Darstellungen visualisiert werden, sondern auch der kollektiven Aushandlung. Obgleich MRT-Visualisierungen keine Summationsbilder sind, erfordert ihre Analyse ein geübtes Gestalterkennen von Organen und Weichteilstrukturen. ${ }^{7}$ Graustufen arbeiten diesem zu, da Kontraste und Details differenzierter wahrgenommen werden können.

Im vorliegenden Fall wird auf Grundlage von MRT-Schnittbildern des Gehirns die Lage eines fraglichen Tumors aus drei Perspektiven beurteilt, mental verräumlicht und der mögliche operative Zugangsweg abgeschätzt. Graustufige Schnittbildserien gelangen jedoch an ihre diagnostischen Grenzen, wo es um Fragen nach den Auswirkungen eines Tumors auf funktionstragende Areale im Gehirn geht. Daher wurden im eingangs skizzierten Fall weitere Verfahren zur Bestimmung von Tumorgrenzen und funktionellen Arealen eingesetzt.

4 Die Soziologin Kelly Joyce hat die Entwicklung der MRT und ihrer nuklearmedizinischen Anwendung aufgearbeitet. Sie stellt fest, dass die numerischen Darstellungen von MRT-Messdaten aufgrund etablierter Bild- und Wahrnehmungskonventionen an die visuellen Präferenzen von Radiologen angepasst wurden. »[T] he practice of printing out both the array of numbers and anatomical pictures ceased soon after NMR technology was placed in radiology units. Instead of representing NMR data as both numbers and images, the data was now solely presented in image form. Developers of MRI recall how radiologists' emphasis on pictures shaped decisions about representation practices. « Joyce 2006.

5 Siehe Hennig 2006, 9; Lynch/Edgerton 1988, 193.

6 Siehe Dommann 1999, 121ff.; Dünkel 2008, 137ff., sowie dies. 2010, 375

7 Siehe Buschhaus 2011, 24; Prasad 2005, $299 f$. 
Verschiedene MRT-Sequenzen, das heißt spezielle Messprotokolle für bestimmte Fragestellungen, erlauben unterschiedliche Detailansichten zur Unterscheidung von gesundem und krankhaftem Gewebe und produzieren komplementäre Bilder ein- und desselben Gehirns. In der T1-Sequenz wird neben der Lage und Größe eines Tumors seine Kontrastmittelaufnahme beurteilt - dies ist ein wichtiger erster Hinweis auf seine Bösartigkeit, da bei malignen Tumoren die Blut-Hirn-Schranke gestört ist und sie eher Kontrastmittel anreichern. Die Ausdehnung von hirneigenen Tumoren wird hingegen häufig in der FLAIR (Fluid-Attenuated Inversion Recovery)Sequenz abgeschätzt, in der das Signal der Hirnflüssigkeit (Liquor) unterdrückt wird und so subtilere Veränderungen in der Flüssigkeitsverteilung bzw. Zelldichte im Gewebe sichtbar werden. Im vorliegenden Fall weist die erhöhte Signalintensität im Bereich des Tumors darauf hin, dass der primäre motorische Kortex stärker involviert sein könnte als zunächst anhand der anderen MRT-Sequenzen angenommen (Abb.2). Durch die tumorbedingte Veränderung der Hirnanatomie ist der funktionelle Bezug zur Motorik allerdings auf Grundlage der bisherigen Bilder nicht sicher beurteilbar. Die navigierte transkranielle Magnetstimulation (nTMS; schmerzlose Reizung des Gehirns mittels Magnetwellen) ermöglicht eine individuelle Kartierung der motorischen Hirnrinde auf Basis der bereits vorhandenen MRT-Bilder: Fokussierte, durch die Schädelkalotte applizierte elektromagnetische Impulse führen zu einer elektrischen Reizung der Hirnrinde - werden motorische Areale stimuliert, wird dies über an den Muskeln von Armen und Beinen angebrachte Klebeelektroden registriert und der Stimulationspunkt mittels der Navigation den MRT-Koordinaten zugeordnet. ${ }^{8}$ Entscheidend sind dabei fundiertes anatomisches Orientierungsvermögen und entsprechende operative Erfahrung, da die ganze Untersuchung anhand einer verhältnismäßig grob aufgelösten, aus MRT-Daten rekonstruierten Oberflächenkarte vorgenommen wird. Im vorliegenden Fall markieren die nTMS-Stimulationen gut nachvollziehbar den Verlauf einer Hirnwindung (Abb.3); in Zusammenschau mit der FLAIR-Bildgebung wird die Infiltration des Motorkortex deutlich, die sich klinisch bei der Erstvorstellung des Patienten bereits angedeutet hatte. Die morphologische Bildkette wurde mithin erfolgreich um eine funktionelle Dimension erweitert.

Derartige Visualisierungen stellen neben ihrer diagnostischen und therapeutischen Relevanz für die Einschätzung und Planung der Neurochirurg_innen zugleich ein wichtiges Instrument für die Kommunikation mit den Patient_innen dar. Im ärztlichen Aufklärungsgespräch, das standardisiert vor Therapiebeginn erfolgt, geht es neben den Risiken des Eingriffs auch um mögliche Folgekomplikationen (etwa motorische Funktionsdefizite) sowie eine prognostische Abschätzung auf Basis der bislang gewonnenen Erkenntnisse. Im dargestellten Patientenfall musste beispielsweise thematisiert werden, dass und warum der Tumor mit Blick auf die funktionellen Untersuchungsergebnisse nicht komplett entfernbar sein würde.

Für die Operationsplanung selbst ergaben sich Konsequenzen für den Zugang, das angestrebte Resektionsausmaß und den Bereich, in dem intraoperativ die Grenzen zwischen entfernbaren und nicht entfernbaren Tumoranteilen mittels navigierter, diesmal direkter elektrischer Stimu-

8 Vgl. Picht 2014 . 


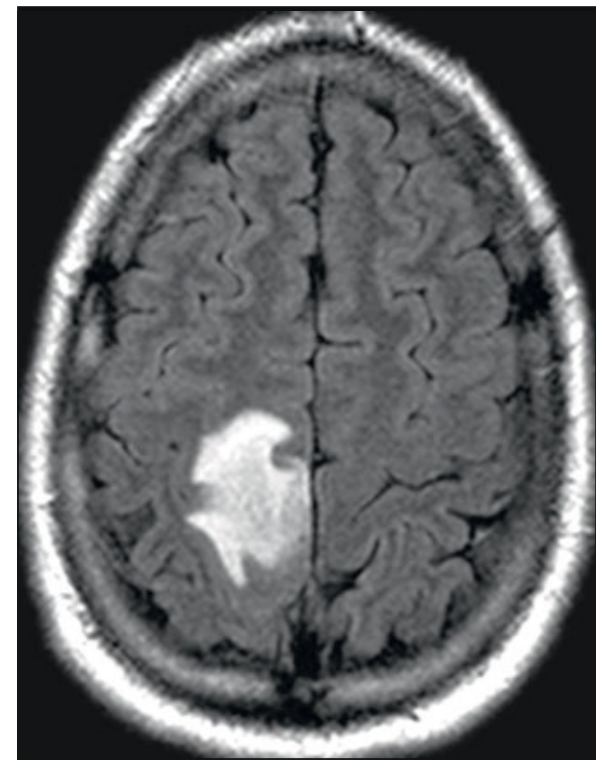

Abb. 2: Präoperatives MRT-FLAIR in axialer Schnittebene (gespiegelt). Das kortikale Tumorareal präsentiert sich in dieser Sequenz größer als in T1.

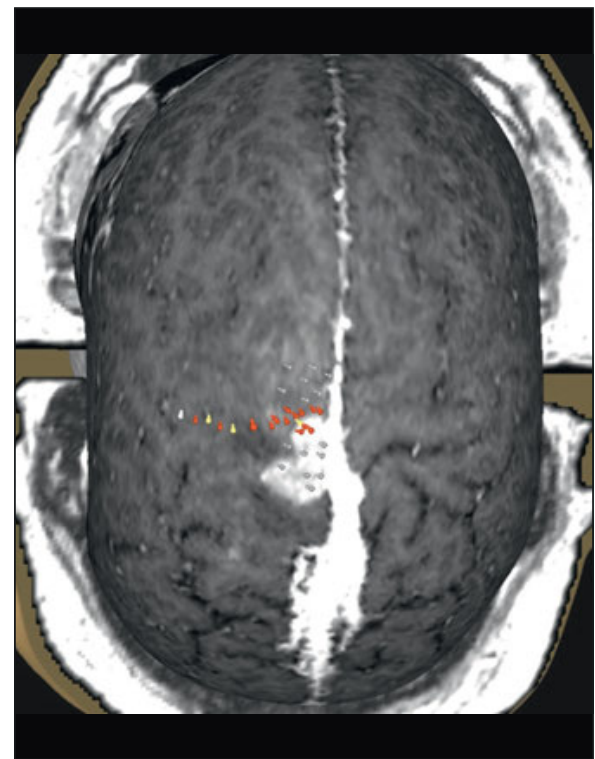

Abb. 3: Präoperativer nTMS-Screenshot in der Untersuchersicht von kranial. Die farbkodierten Punkte zeigen die gemessene Intensität der stimulierten motorischen Reaktion (weiß = stark, gelb $=$ mittel, rot $=$ gering, grau = keine Reaktion).

lation bestimmt werden mussten. Die MRT-Bilder des Patienten, die Faserbahndarstellung der weißen Hirnsubstanz - das sogenannte Fibertracking (Diffusion Tensor Imaging, DTI) - und die nTMS-Ergebnisse wurden für die OP-Planung und -Durchführung fusioniert und in einer speziellen Planungssoftware (Brainlab iPlan Cranial 3.0) weiterbearbeitet, um anschließend in eine Navigationssequenz für die Operation überführt zu werden.

Farblich entsprechend kodiert, sind im präoperativen Planungsbild der Tumor (rot), die Infiltrationszone (grün), die motorischen Stimulationspunkte (gelb) und davon ausgehend der Verlauf der Pyramidenbahn, der wichtigsten motorischen Bahn (blauviolett), zu sehen (Abb.4). Aus den drei Schnittebenen lässt sich eine 3D-Simulation des Patientenkopfes rekonstruieren, in der die Abstände des Tumors zu relevanten Strukturen, die geschont werden müssen, besser beurteilbar sind. Die Grundlage für die gesamte Planungssequenz bildet erneut das MRT-Bild, das zu Beginn zur Diagnosefindung erstellt wurde, nun jedoch bereits eine Reihe von operationalen Metamorphosen durchlaufen hat und in zunehmendem Maße die morphologische Basis für den eigentlichen Eingriff darstellt - es wird zu einer dreidimensionalen Karte, in die alle anderen Informationen eingetragen werden können, und bildet auf diese Weise einen eigenen 3D-Planungs- und Navigationsraum. 


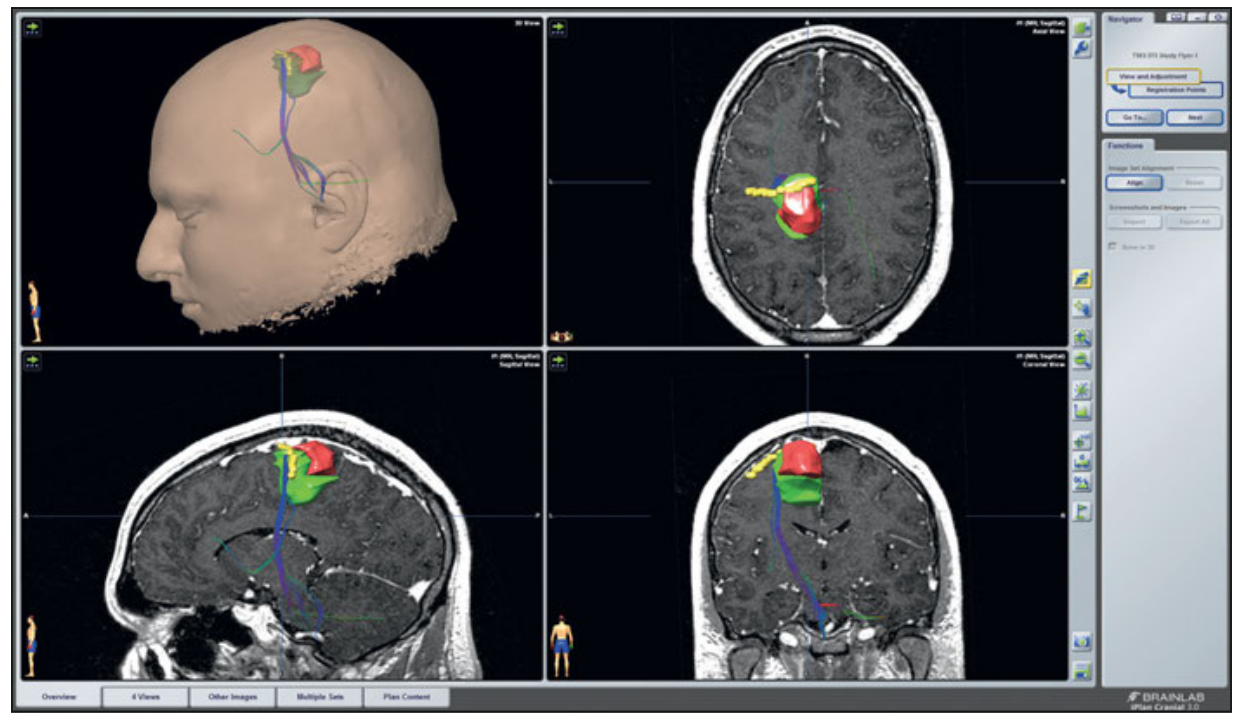

Abb. 4: Präoperative Operationsplanung in der Software Brainlab iPlan Cranial 3.o. Links oben der rotierbare dreidimensionale Planungskopf; im Uhrzeigersinn das axiale, koronare und sagittale Fusionsbild aus MRT-Sequenzen, DTI- und nTMS-Daten.

Ein solches 3D-Modell ermöglicht allerdings nicht in jeder Hinsicht eine größere Konkretion als die zweidimensionalen Schnittbilder, etwa wenn es darum geht, einzelne Gewebeschichten zu differenzieren. Wo liegen nun die raumbezogenen Vorteile dieses Modells? Die Planungssoftware ermöglicht in zunehmendem Maße, die mentale Rekonstruktion des Operateurs oder der Operateurin aus der reinen Schnittbildgebung in ein Modell zu übersetzen, das im Idealfall frei rotationsfähig ist und alle relevanten Strukturen in eine transparente Kopfdarstellung einblenden kann. Dies erlaubt eine gezielte Analyse der Lagebeziehung zwischen Tumor und Risikostrukturen. Genau hierin liegt aber auch das Problem: Gegenwärtige 3D-Simulationsmodelle konfligieren mit den auf Erfahrung und visueller Kognitionsleistung basierenden mentalen Rekonstruktionen der Operateur_innen. Sie können die Planung am Schnittbild allenfalls ergänzen. Hier ist noch viel ungenutztes Potenzial vorhanden, die Chirurg_innen visuell umfassender zu unterstützen. Auch Patient_innen kann man an einem entsprechenden inividualisierten Planungsmodell den bevorstehenden Eingriff im Detail besser vermitteln. Die Übersetzung der einzelnen Planungsetappen auf den Patient_innenkörper ist der letzte Schritt, der für die anschließende Operation notwendig ist. Alle ermittelten Daten werden mit dem Operationsplan zur Leitung und Kontrolle in die intraoperative Navigation übernommen und stehen dann am erwähnten Patienten zur Verfügung. Der Anwendungsbereich von Visualisierungspraktiken beschränkt sich also nicht nur auf die Diagnostik, Planung und Überwachung, sondern setzt sich in den Behandlungsprozess hinein ununterbrochen fort. 
Diese Ausweitung und Integration der medizinischen Bildkette in die Therapie lassen sich besonders anschaulich anhand der neurochirurgischen Operationssaal-Architektur nachvollziehen. Sie konfrontiert Chirurg_innen mit einer Vielzahl bildgebender Verfahren und visueller Darstellungen, die sie vor die Herausforderung stellen, ein zunehmend komplexes Gefüge von Bildtechniken und -informationen angemessen in ihre Handlungen zu integrieren (Abb. 5).

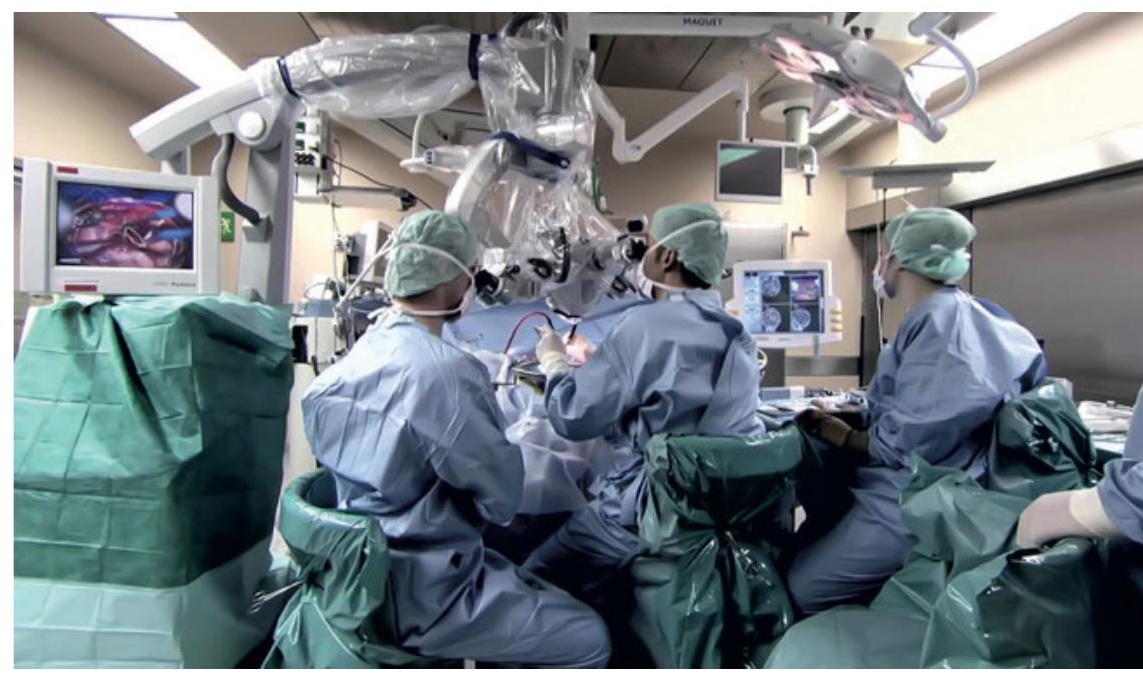

Abb. 5: Intraoperatives Setting mit Mikroskop (Mitte), Mikroskopbildschirm (links) und Navigationsbildschirm (rechts hinten).

Um den Einfluss dieser Bilder auf die neurochirurgische Praxis nachzuvollziehen, ist nicht nur die Analyse der perzipierbaren Oberfläche ausschlaggebend, wie etwa die Gestaltungselemente eines visuellen Interfaces. Vielmehr müssen dazu ebenso die visuelle Architektur und die softwaretechnische Funktionsweise bildgebender Verfahren in den Blick genommen werden. Erst am Zusammenspiel dieser drei Faktoren lässt sich zeigen, unter welche operativen Bedingungen die Bildtechnik das ärztliche Sehen und Handeln stellt. So wirkt die Anordnung von Eingabegeräten und Displays handlungsstrukturierend, ${ }^{9}$ etwa wenn die Positionierung von Bildschirmen ergonomische Blickachsen ermöglicht oder verstellt. ${ }^{10}$ Ebenso determinieren Softwareprogramme die Modalitäten von Interaktion, etwa indem sie durch die Modellierung visueller Informationen überhaupt erst definieren, was sichtbar gemacht werden kann." Wie wird dieses komplexe Gefüge von Bildtechniken und -informationen in den chirurgischen Workflow integriert, und welche Handlungsmöglichkeiten und -defizite ergeben sich daraus?

9 Siehe Wasen/Brierley 2013.

10 Siehe Sielhorst/Feuerstein/Navab 2008.

11 Siehe Hinterwaldner 2014. 
Der Blick durch das Mikroskop allein ist nicht ausreichend, um einen Tumor zu lokalisieren und die Instrumente unter Schonung der Risikostrukturen entsprechend zu navigieren. Erst die Integration der im Vorhinein erstellten Navigationssequenz in die intraoperative Situation ermöglicht die Orientierung im Operationsgebiet: Planung und Durchführung werden so weit wie möglich kongruent. Mithilfe eines Trackingsystems werden dazu die Positionen von Mikroskop und Instrumenten in Echtzeit mit den präoperativen MRT-Bildern und der intraoperativen Lage des Kopfes fusioniert. Diese Fusion von 3D-Raumkoordinaten und 2D-Schnittbildern wird auf einem separaten Navigationsbildschirm dargestellt, der mittels beweglicher Schwenkarme an die Betrachtersituation der Chirurg_innen angepasst werden kann (Abb.6). Erst diese Darstellung ermöglicht einen Überblick und damit den strukturierten Zugriff auf das Operationsgebiet. Das heißt, erst wenn die Raumkoordinaten von Instrument, Körper und Bild auf dem Navigationsbildschirm zur Deckung gebracht werden, kann die notwendige Orientierung im Operationsgebiet gewährleistet werden. Im Gegensatz zum Blick durch das Mikroskop, der nur den sichtbaren Teil der Anatomie der sonst abgedeckten Patient_innen erfasst, erschließt der Blick auf den Navigationsbildschirm die globale Lage des jeweiligen Tumors. Der Blick der Chirurg_innen muss dementsprechend immer wieder zwischen Navigationsbildschirm und Mikroskopansicht hin und her wechseln.

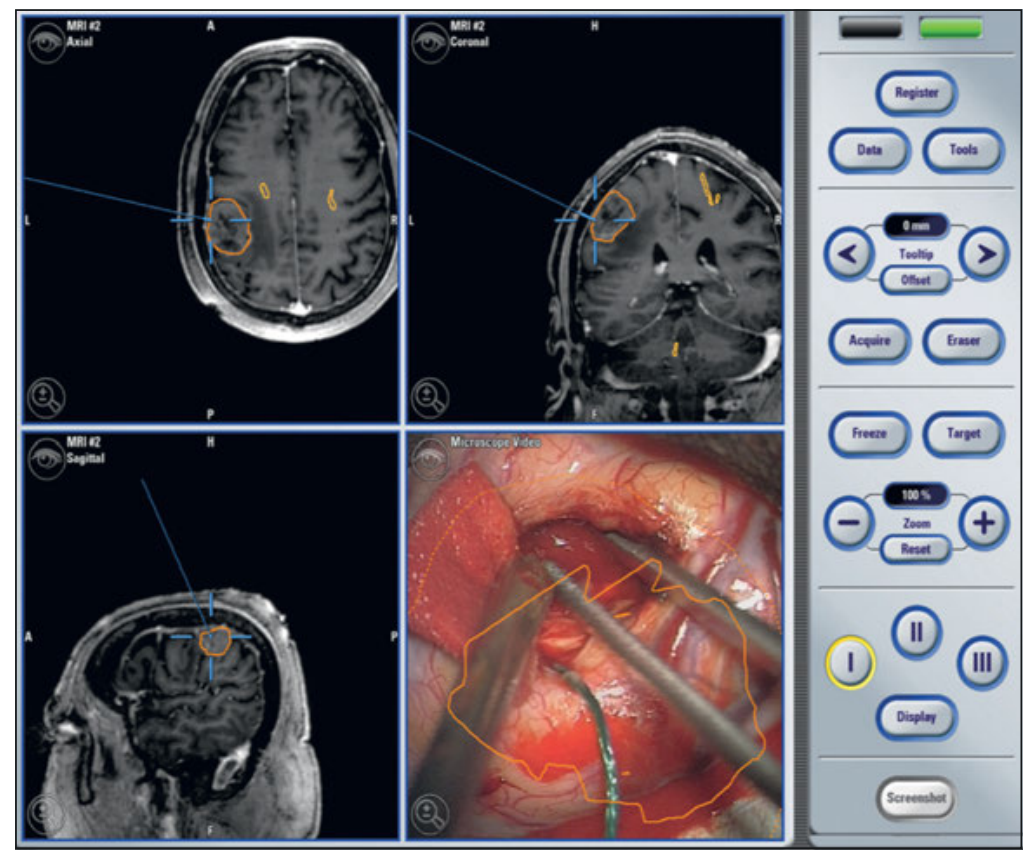

Abb. 6: Intraoperativer Screenshot des Navigationsbildschirms (Brainlab Vector Vision). 
Auffällig ist hier, dass die Bilder auf dem Navigationsbildschirm den Kopf des Patienten oder der Patientin dabei nicht etwa aus der Perspektive der Chirurg_innen zeigen, sondern als multiplanare Rekonstruktion, also in je einer Längs-, Quer- und Aufsicht, die zwar auf die jeweilige Behandlungssituation ausgerichtet werden kann (beispielsweise durch ein Vertauschen von links und rechts), hingegen nicht der tatsächlichen Blickrichtung der Chirurg_innen oder der Ausrichtung des Patienten oder der Patientin entsprechen. Die Chirurg_innen müssen diese unterschiedlich perspektivierten Darstellungen ebenso in ihren jeweiligen Arbeitsablauf integrieren wie die räumliche Anordnung des Navigationsbildschirms und die dadurch entstehenden Blickwechsel zwischen Bild und Operationsgebiet. Was ohnehin schon eine komplexe Abstraktionsleistung ist, nämlich die kognitive Überbrückung der Differenz zwischen Auge und Aufzeichnung, wirkt sich nachteilig auf die Hand-Auge-Koordination der Chirurg_innen aus: Die Führung von Instrumenten sowie die Orientierung im Operationsgebiet werden durch die unzureichende Anpassung von Bild und Blick erschwert. ${ }^{12}$ Neurochirurgische Mikroskope unterstützen bereits Bildmodi, die das handlungsbedingte Nebeneinander von Formen der Beobachtung und Repräsentation, von Bild und Körper, von Bildschirm und Operationsgebiet, von Bildfläche und Operationsraum überwinden. Dabei wird das intraoperative Kamerabild des Mikroskops mit präoperativen 3D-Modellen fusioniert, womit eine maßgebliche Reduzierung des Abstraktionsschrittes zwischen Bild und Handlung erreicht wird.

Prinzipiell wird in allen chirurgischen Fächern inzwischen eine Echtzeit-Überlagerung bildgebender Daten mit dem Patient_innenkörper angestrebt (Abb. 7). Das Hauptproblem dabei ist jedoch, dass sich die Anatomie während jeden invasiven Eingriffs unter der chirurgischen Manipulation verändert, etwa in Folge von sogenannten Brainshifts. Somit verlieren die präoperativ erhobenen Daten in der konkreten chirurgischen Situation schnell ihre Genauigkeit. Derzeit wird diese Form der Navigation deshalb nur als unterstützende Modalität genutzt, um intraoperativ die bestmöglichen Entscheidungen zu treffen.

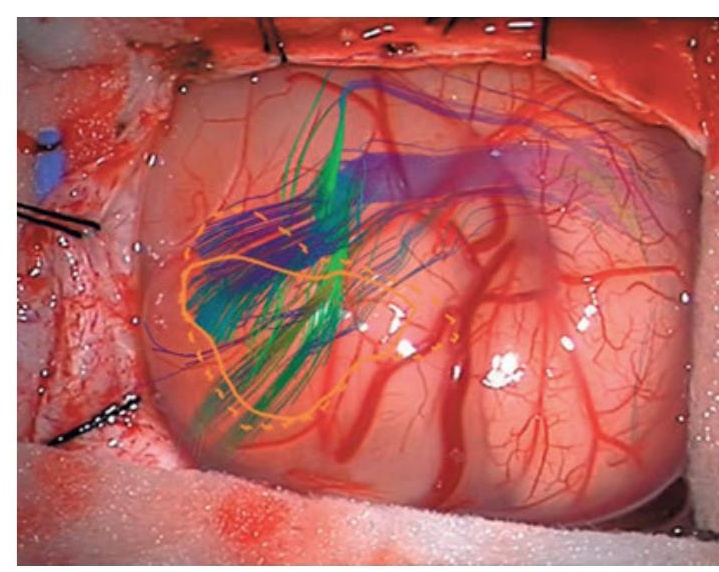

Abb. 7: Intraoperativer Screenshot des navigierten Mikroskops. 


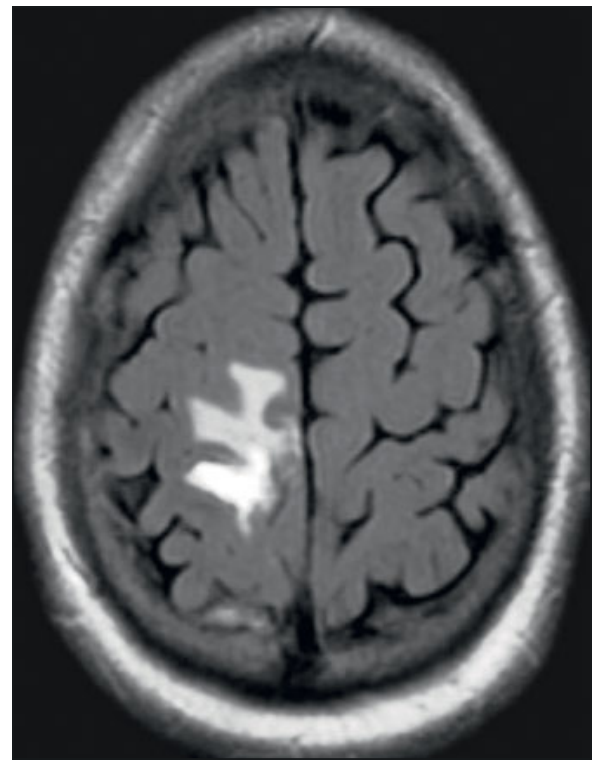

Abb. 8: Postoperatives MRT-FLAIR in axialer Schnittebene (gespiegelt). Das kortikale Tumorareal ist deutlich reduziert. In Weiß ist die flüssigkeitsgefüllte Resektionshöhle zu sehen, davor in Hellgrau der nicht entfernbare funktionstragende Tumoranteil.
Verlässlichen Aufschluss über die Qualität des Behandlungsergebnisses ermöglicht letztlich erst die postoperative Bildgebung, mit der die MRT-Bildkette zu einem vorläufigen Abschluss kommt (Abb. 8). Ziel muss folglich eine Echtzeit-Überlagerung sein, die kontinuierlich an die sich verändernden anatomischen Verhältnisse angepasst wird.

\section{Fazit}

In der aktuellen Medizin haben sich die Fragen an die Bilder vervielfacht. Während grundsätzliche Interessen am Bild die gleichen geblieben sind (Bestätigung oder Widerlegung einer Verdachtsdiagnose, genaue Lokalisation und Ausmaß der Erkrankung, Vorabplanung der therapeutischen Interventionen, Verlaufskontrolle), hat sich der Umfang der Bildarbeit aufgrund der neuen technischen Möglichkeiten in den letzten 20 Jahren stark erweitert.

Aus praktisch-chirurgischer Sicht bleibt festzustellen, dass der technologische Fortschritt im Bereich der Bilddatengenerierung und -darstellung der letzten Jahre bis dato nur unzureichend in die konkrete Behandlungssituation übersetzt wurde.

Die Synchronisierung zwischen Körper und Bild stellt nicht nur für die Medizintechnik eine der zentralen Herausforderungen dar, sondern erfordert ebenso die Überwindung von Sehgewohnheiten in der chirurgischen Praxis. Dort dominiert nach wie vor das diagnostische radiologische Sehmodell zweidimensionaler Schnittbilder, obwohl die Navigation anhand entsprechender 3D-Modelle eine zusätzliche Raumorientierung ermöglicht, die effektiver an die Blickrichtung und Handlungsposition des Chirurgen anpassbar wäre. Diese neuen Anschauungssituationen konfrontieren Neurochirurg_innen mit einer neuen epistemischen Herausforderung: Die Schwierigkeit besteht hier nicht darin, die Differenz zwischen Körper und Bild zu eliminieren bzw. kognitiv zu überbrücken, sondern gerade in der praktischen Kenntnis der Unterscheidung zwischen Körper und Bild. Daneben steht die konstant erforderliche Evaluierung technologischer Möglichkeiten und Limitierungen durch die Ärzt_innen in einem grundsätzlichen Konflikt mit zeiteffizienten Arbeitsroutinen: Im Spannungsfeld zwischen der Entwicklung in der Medizingeräte-Industrie und der Anwendung in der Klinik stellt die bild- und medienwissenschaftliche Kritik womöglich einen entscheidenden Ansatz dar, um technischen Fortschritt auch zum Wohle der Patient_innen optimal in die klinische Praxis zu übersetzen. 


\section{Literatur}

Alač, Morana (2011): Handling Digital Brains. A Laboratory Study of Multimodal Semiotic Interaction. Cambridge, MA: MIT Press.

Beaulieu, Anne (2002): Images Are Not the (Only) Truth: Brain Mapping, Visual Knowledge, and Iconoclasm. In: Science, Technology, and Human Values, vol. 271, no. 1 pp. $53-86$.

Bruzzone, Maria Grazia et al. (2012): CT and MRI of Brain Tumors. In: The Quarterly Journal of Nuclear Medicine and Molecular Imaging, vol. 56, no. 2, pp. 112-137.

Burri, Regula Valérie (2008): Doing Images. Zur Praxis medizinischer Bilder. Bielefeld: transcript.

Buschhaus, Markus (2011): Körperwelten in Graustufen. Zu den Anfängen der Computertomografie. In: Bredekamp, Horst/Bruhn, Matthias/Werner, Gabriele (Hg.): Bildwelten des Wissens. Kunsthistorisches Jahrbuch für Bildkritik, Bd. 8.2: Graustufen. Berlin: Akademie Verlag, S. 19-27.

Dommann, Monika (1999): »Das Röntgen-Sehen muss im Schweiße der Beobachtung gelernt werden.«Zur Semiotik von Schattenbildern. In: Traverse, Jg. 6, Nr.3, S. 114-130.

Dünkel, Vera (2008): Röntgenblick und Schattenbild. Zur Spezifik der frühen Röntgenbilder und ihren Deutungen um 1900. In: Bredekamp, Horst/Schneider, Birgit/Dünkel Vera (Hg.): Das Technische Bild. Kompendium zu einer Stilgeschichte wissenschaftlicher Bilder. Berlin: Akademie Verlag, S. 136-147.

Hanna, G./Shimi, S./Cuschieri, A. (1998) Task Performance in Endoscopic Surgery Is Influenced by Location of the Image Display. In: Annals of Surgery, vol. 227, no.4, pp. $481-484$.

Hennig, Jürgen (2006): Farbeinsatz in der medizinischen Visualisierung. In: Bredekamp, Horst/Bruhn, Matthias/ Werner, Gabriele (Hg.): Bildwelten des Wissens. Kunsthistorisches Jahrbuch für Bildkritik, Bd. 4.1: Farbstrategien, Berlin: Akademie Verlag, S. 9-16.

Hinterwaldner, Inge (2014): Tiefenschichtung der Gestaltung/Deep Layers of Design. In: Texte zur Kunst, Nr. 95: Bild vs. Kunst, S. 156-167 (dt./engl.).
Joyce, Kelly (2006): From Numbers to Pictures: The Development of Magnetic Resonance Imaging and the Visual Turn in Medicine. In: Science as Culture, vol. 15 no. 1, pp. 1-22

Joyce, Kelly (2008): Magnetic Appeal. MRI and the Myth of Transparency. Ithaca: Cornell University Press.

Lynch, Michael/Edgerton, Samuel Y. (1988): Aesthetics and Digital Image Processing. Representational Craft in Contemporary Astronomy. In: Fyfe, Gordon/Law, John (eds.): Picturing Power. Visual Depictions and Social Relations. London: Routledge, pp. 184-220.

Mentis, Helena/Chellali, Amine/Schwaitzberg, Steven (2014): Learning to See the Body: Supporting Instructional Practices in Laparoscopic Surgical Procedures. In: Proceedings of the ACM CHI Conference on Human Factors in Computing Systems. New York: ACM, pp. 2113-2122.

Picht, Thomas (2014): Current and Potential Utility of Transcranial Magnetic Stimulation in the Diagnostics before Brain Tumor Surgery. In: CNS Oncology, vol. 3, no. 4 , pp. $299-310$

Prasad, Amit (2005): Making Images/Making Bodies. Visibilizing and Disciplining through Magnetic Resonance Imaging (MRI). In: Science, Technology, and Human Values, vol. 30, no. 2, pp. 291-316.

Sielhorst, Tobias/Feuerstein, Marco/Navab, Nassir (2008): Advanced Medical Displays: A Literature Review of Augmented Reality. In: Journal of Display Technology, vol. 4, no. 4, pp. 451-467.

Wasen, Kristian/Brierley, Meaghan (2013): The Visual Touch Regime: Real-Time ${ }_{3} D$ Image-Guided Robotic Surgery and ${ }_{4} D$ and $\gg 5 D$ « Scientific Illustration at Work. In: Wasen, Kristian (ed.): Emerging Health Technology: Relocation of Innovative Visual Knowledge and Expertise. Heidelberg/New York: Springer, pp. 21-51. 


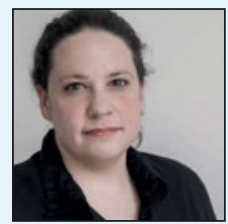

kathrin.friedrich@hu-berlin.de

Wissenschaftliche Mitarbeiterin

Basisprojekt: Image Guidance

Disziplin: Medienwissenschaft

Kathrin Friedrich ist wissenschaftliche Mitarbeiterin im Exzellenzcluster Bild Wissen Gestaltung. Zuvor hat sie am Lehrstuhl für Ästhetik der Kunsthochschule für Medien Köln und im BMBF-Verbundprojekt Verkörperte Information. >Lebendige< Algorithmen und zelluläre >Maschinen< gearbeitet. Dort 2015 Verteidigung der medienwissenschaftlichen Dissertationsschrift Medienbefunde. Digitale Bildgebung und diagnostische Radiologie. Sie studierte Medienwissenschaft, Rechtswissenschaften und Soziologie an der Philipps-Universität Marburg. Ihre Forschungsschwerpunkte sind bildgeführte Interventionen in der Medizin, Software Studies sowie Computer-Aided Design und Serious Gaming in der Biologie.

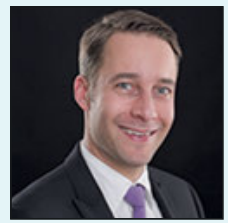

thomas.picht@charite.de

Principal Investigator

Basisprojekt: Image Guidance

Disziplin: Medizin

Thomas Picht ist Neurochirurg und Oberarzt in der Neurochirurgie an der Charité Berlin. Er leitet seit vielen Jahren die AG Perioperative Funktionsdiagnostik der Neurochirurgischen Klinik und war maßgeblich an der Einführung und Weiterentwicklung der navigierten transkraniellen Magnetstimulation (nTMS) zur präoperativen Diagnostik und Operationsplanung bei Hirntumoren beteiligt. Im Exzellenzcluster Bild Wissen Gestaltung bringt er vor allem seine umfassende klinische Erfahrung mit Problemen der Bildnavigation, Bildfusion und bildgestützter Operationsplanung ein. 


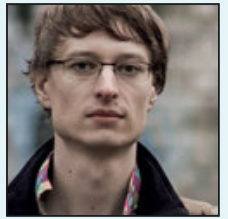

moritz.queisner@hu-berlin.de

Wissenschaftlicher Mitarbeiter

Basisprojekt: Image Guidance

Disziplin: Medienwissenschaft

Moritz Queisner ist Medienwissenschaftler und wissenschaftlicher Mitarbeiter im Exzellenzcluster Bild Wissen Gestaltung sowie Mitglied im Graduiertenkolleg Sichtbarkeit und Sichtbarmachung. Hybride Formen des Bildwissens an der Universität Potsdam. Er war zuvor wissenschaftlicher Mitarbeiter in der Kolleg-Forschergruppe Bildakt und Verkörperung an der Humboldt-Universität zu Berlin und im Post Media Lab am Center for Digital Cultures der Leuphana Universität Lüneburg. Seine aktuellen Forschungsschwerpunkte sind Augmented Reality, Drohnen und Geomedien.

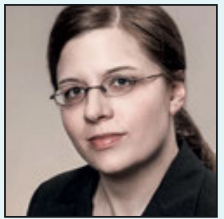

anna.roethe@hu-berlin.de

Wissenschaftliche Mitarbeiterin

Basisprojekte: Gesundheit \& Gestaltung, Image Guidance

Disziplin: Kulturwissenschaft, Medizin

Anna L. Roethe ist Ärztin und Kulturwissenschaftlerin. Sie verfolgt ihre interdisziplinären Forschungsinteressen - unter anderem visuelle Epistemik in medizinischer Bildgebung und chirurgischen Interventionen, medizinische Narrative in Klinik, Kunst und Populärkultur, Wissensgestaltung in der Beziehung zwischen Ärzt_innen und Patient_innen - als wissenschaftliche Mitarbeiterin in den Basisprojekten Gesundheit \& Gestaltung und Image Guidance im Exzellenzcluster Bild Wissen Gestaltung sowie im Operationssaal der Charité Berlin. 


\section{Wissenschaftliche Sachcomics}

\section{Multimodale Bildsprache, partizipative Wissensgenerierung und raumzeitliche Gestaltungsmöglichkeiten}

\section{Sachcomics als Slow Media}

Unter Slow Media ${ }^{7}$ sind nichtlineare Kommunikationsformate zu verstehen, welche zu ihrer Wissenserschließung einer umfassenden persönlichen Beschäftigung der Leser_innen oder Betrachter_innen bedürfen. Das Medium muss in Raum und Zeit verortet werden und gleichzeitig verschiedene Sinne direkt bzw. über synästhetische Ansätze ansprechen. ${ }^{2}$ Insbesondere können Abbildungen und Sammlungsobjekte, aber auch komplette nicht linear strukturierte Ausstellungen als Slow Media betrachtet werden. ${ }^{3}$ Im Sinne von Slow Media lassen sich auch Sachcomics zu komplexen Themen grundsätzlich mit wissenschaftsbasierten Ausstellungen vergleichen, da sie dominant visuell gestaltet sind, Informationen zu Mehrebenennarrativen verbinden, individuelle Geschwindigkeit beim Erfassen erlauben und damit gleichzeitig erhöhte > partizipative< Aktivität beim Zusammensetzen der Informationen und Themen im Kopf erfordern. ${ }^{4}$ Comics sind hierbei insbesondere durch ihre Bildsprache motivierend, visualisierend, permanent, intermediär und populär..$^{5}$ Die szenografische Gestaltung erlaubt die Kombination

1 Siehe David/Blumtritt/Köhler 2010.

2 Siehe McCloud 1993; ders. 2014; Plank 2013; Sousanis 2015.

3 Siehe Robin et al. 2014; Leinfelder 2015.

4 Vgl. Jacobs 2007; Leinfelder 2014; ders. 2014a; ders. 2015; Groensteen 2014; Sousanis 2015.

5 Vgl. Versaci 2001; Morrison/Bryan/Chilcoat 2002; Yang 2008. 
realitätsnaher (wahrnehmbarer), abstrahierter (zu erfassender) und symbolisierter (erlernter) Bilder in teils vielschichtig angelegten Panels, welche zeitlich, räumlich und perspektivisch insbesondere durch Weglassen (in den > Gutters<, Lücken zwischen Panels) inszeniert werden können. ${ }^{6}$ Je nach Komplexität der Themen können sich Text und Bildsequenzen komplementär oder auch einander stützend verhalten. ${ }^{7}$ Bei komplexen Sachcomics sollten emotionale Elemente, sowohl in Wort als auch im Bild, zwar verwendet, insgesamt jedoch eher zurückhaltend eingesetzt werden. Hingegen sind Personalisierung, Darstellung wissenschaftlicher Arbeitsweisen, Visualisierung von Szenarien und andere Authentifizierungsmöglichkeiten wissenschaftlichen Vorgehens genauso wie mögliche gesellschaftliche Relevanzen und Handlungsoptionen teilweise augenzwinkernd gestaltbar, womit sich die zu vermittelnden Themen von ihrer gewissen >Schwere< befreien lassen. Dies kann zum Beispiel durch Zuhilfenahme von >Sidekicks< bzw. durchlaufenden Nebengeschichten erreicht werden. Insbesondere sind auch gesellschaftlich herausfordernde Themen bzw. Handlungsoptionen, welche bei der Verwendung anderer Medien sehr schnell zu reflexartiger Ablehnung führen, bei Comics durch die vielfältigen Möglichkeiten der humoristischen Einfärbung besser transportierbar. ${ }^{8}$

\section{Aktivitäten}

Eigene Aktivitäten hierzu umfassen ein als Comic gestaltetes Interview zu Biodiversitätsthemen ${ }^{9}$ und vor allem die Umsetzung eines kompletten Gutachtens des Wissenschaftlichen Beirats der Bundesregierung Globale Umweltveränderungen (WBGU) zum Klimawandel ${ }^{10}$ in einen als sogenannte Graphic Interviews realisierten Sachcomic," welcher selbst umfassend theoretisch und empirisch beforscht wurde (Abb. 1)..$^{12}$

In einem weiteren Projekt wurden Comic Strips zu Sammlungsobjekten in eine Ausstellung integriert, mit dem Ziel, Objekte der Dauerausstellung mit einer aktuellen Sonderausstellung zu verknüpfen - auch hier ging es um ein außerordentlich komplexes Thema: das Anthropozän. ${ }^{13}$ Die Bildgeschichten erfordern nicht nur eine eigene comicspezifische Beteiligung und Kontextualisierung durch die Leserin oder den Leser, sondern wurden zu einem guten Teil auch partizipativ erstellt (Abb. 2). Die Illustrator_innenklasse von Hennig Wagenbreth an der Universität der Künste Berlin setzte sich intensiv mit den vorausgewählten Ausstellungsobjekten auseinander und erarbeitete deren Bezug zum Anthropozän. Um einen formalen Wiedererkennungswert in

6 Siehe McCloud 1993; ders. 2014.

7 Siehe Jüngst 2010.

8 Vgl. Brocka 1979; Hangartner/Keller/Oechslin 2013; Leinfelder 2014; ders. 2014 a.

$9 \mathrm{Vgl}$. Hamann/Feindt/Leinfelder 2011.

10 Siehe WBGU 2011.

11 Siehe Hamann/Zea-Schmidt/Leinfelder 2013; dies. 2014.

12 Siehe Leinfelder 2014; ders. 2014a.

13 Sonderausstellung im Deutschen Museum, München, vom 5. Dezember 2014 bis 31. Januar 2016. 


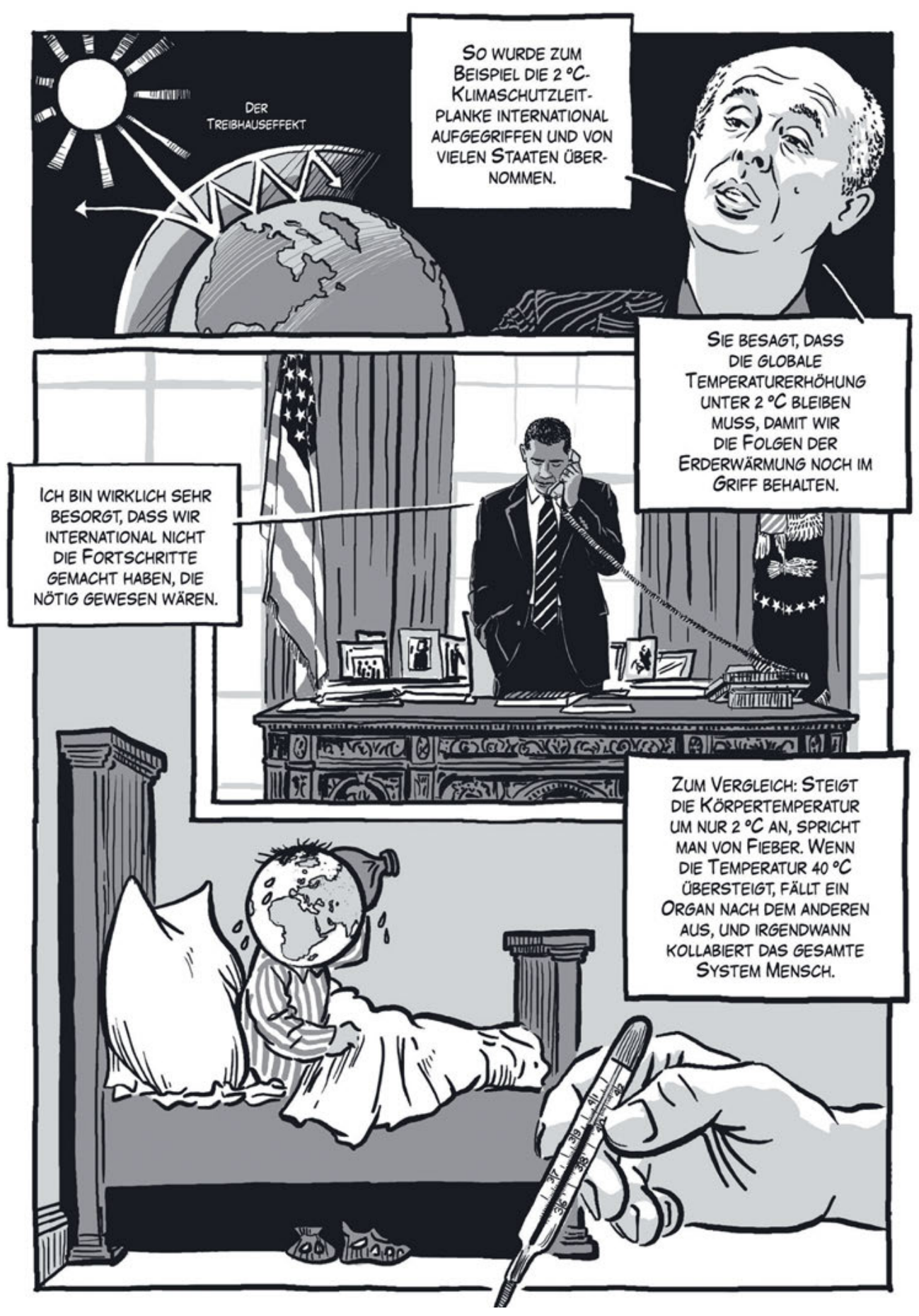

Abb. 1: Beispiel für einen wissenschaftlichen Sachcomic ist Die große Transformation. Klima - Kriegen wir die Kurve? Hier ein Ausschnitt aus dem Kapitel Warum wir uns transformieren müssen mit John Schellnhuber, Leiter des Potsdam-Instituts für Klimafolgenforschung, gezeichnet von Studio Nippoldt. 
der Ausstellung und ein einheitliches grafisches Design in der dazugehörigen Buchausgabe zu erreichen, wurde den Comic Strips ein striktes Format vorgegeben. Die gezeichneten Geschichten mussten in jeweils acht gleichgroßen Panels erzählt werden, ferner waren nur Schwarz und eine Sonderfarbe zugelassen. Das Ergebnis ist ein in dieser Form sehr unerwartetes Kaleidoskop von persönlichen Narrativen, welche die Bandbreite der Möglichkeiten von Comics gut abbilden und sowohl Wissenschaft als auch Technik in einen sehr persönlichen, teils gesellschaftskritischen Kontext stellen, der zur Reflexion animieren soll. ${ }^{14}$

Anhand dieser innerhalb wissenschaftlicher Projekte erstellten Sachcomics können Ableitungen destilliert werden: So ergaben empirische Untersuchungen, dass im deutschsprachigen Raum Comics vorurteilshaft oft noch als »flach « oder »unseriös « eingestuft werden. ${ }^{15}$ Diese Wahrnehmung verkennt die Geschichte sowie die aktuelle Vielfalt der Sachcomics und ist teilweise damit zu erklären, dass Deutschland, etwa im Unterschied zu den USA, Frankreich oder Japan, über eine nur mäßig ausgeprägte historische Comic-Kultur verfügt und hier meist nur wissenschaftsfiktionale Comics (Daniel Düsentrieb, Superheroes) bekannt sind..$^{16}$ Auch deshalb müssen die Ansprüche an Authentizität und Dokumentation für wissenschaftliche Sachcomics ähnlich hoch wie für wissenschaftliche Veröffentlichungen und damit höher als für sehr gut recherchierte klassische journalistische Formate sein. ${ }^{17}$ Unter Berücksichtigung dieser Aspekte haben Sachcomics ein enorm breites Potenzial für kreativen Wissenstransfer sowie für Gestaltungs- bzw. Handlungsmotivation. Darüber hinaus können sie auch als partizipativer, dialogischer und gestaltender Prozess für die Generierung wissenschaftlicher Ansätze und für die Identifizierung neuer Forschungsnotwendigkeiten konzipiert werden. Letzteres wird nachfolgend anhand des interkulturellen und partizipativen Comic-Vorhabens des Basisprojektes Die Anthropozän-Küche näher ausgeführt.

\section{Der Anthropozän-Küchencomic - ein global partizipativer und transdisziplinärer Ansatz zu Wissensgenerierung und Wissensgestaltung}

Theoretischer Ansatz: Wissenschaftliches Arbeiten zur Reflexion und Lösung von Zukunftsaufgaben muss wegen der Komplexität der Thematiken, der Verknüpfung von Analysen, Szenarien und Handlungsoptionen sowie deren gesellschaftlicher Relevanz nicht nur interdisziplinär, sondern insbesondere auch transdisziplinär angelegt sein. ${ }^{18}$ Transdisziplinäre Forschung umfasst eine gesellschaftliche Beteiligung am Forschungsprozess und an der Umsetzung von Lösungsvorschlägen. ${ }^{19}$ Eine transdisziplinäre Herangehensweise definiert gemeinsam Probleme und Untersuchungswege (Co-Design) und ermöglicht eine Wissensintegration mit dem Wissen der

14 Vgl. Hamann u. a. 2014.

15 Leinfelder 2014.

16 Vgl. Brocka 1979; Grünewald 2014.

$17 \mathrm{Vgl}$. Jüngst 2010; Plank 2013.

18 Siehe WBGU 2011; Leinfelder 2013.

19 Siehe WBGU 2011. 

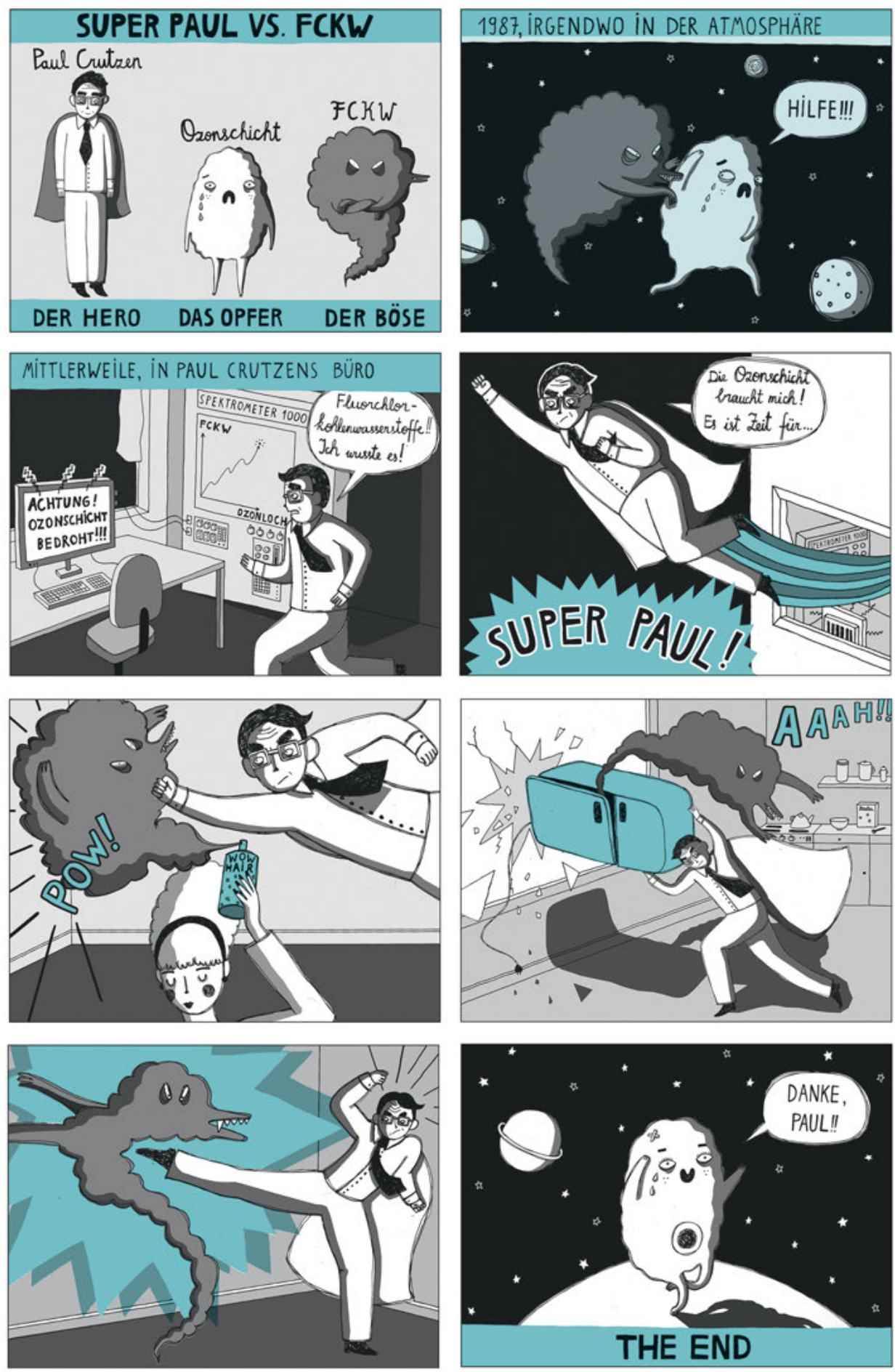

Abb. 2: Ausschnitt aus der Comic-Anthologie Anthropozän - 30 Meilensteine auf dem Weg in ein neues Erdzeitalter: M.I.P.A.S., Paul J. Crutzen als Super-Paul, gezeichnet von Martyna Zalalyte. 
Anwender_innen (Co-Production). Laut Schneidewind und Singer-Brodowski umfasst Transdisziplinarität neben den Prinzipien des Co-Designs und der Co-Production im Wissenschaftsprozess auch eine neue Dimension der Verantwortung für Wissenschaftler_innen. ${ }^{20}$ Gemeinsam gestaltete Realexperimente (Experimental Turn) stellen hierbei einen wichtigen Erkenntnismodus der transformativen Wissenschaften dar. Diese werden in sogenannten Reallaboren, also in der Regel im offenen Raum, durchgeführt, um in einem gesellschaftlichen Kontext soziale Dynamiken und Prozesse erfahrbar und erforschbar zu machen.

Hierzu ist es auch notwendig, die Trennung zwischen wissenschaftlicher Wissensproduktion und Wissenschaftskommunikation aufzuheben. ${ }^{21}$ Gerade beim Thema Essen und Ernährung sind diese Ebenen eng über das Erfahrungswissen miteinander verknüpft, welches seinerseits stark kulturell geprägt ist. Am Exzellenzcluster Bild Wissen Gestaltung wird dazu - unseres Wissens weltweit erstmalig - ein sachcomicbasiertes Co-Design sowie eine daraus resultierende Forschungs-Co-Production mit der Zivilgesellschaft in globalem Maßstab durchgeführt. Die gemeinsame Erarbeitung der Narrative mit gesellschaftlichen Protagonist_innen aus zehn Ländern zum Rahmenthema Essen und Ernährung hat dabei zum Ziel, aus einem dialoggesteuerten Co-Design den weiteren wissenschaftlichen Recherchebedarf zu generieren und infolgedessen zu unerwarteten Rekombinationen, Verknüpfungen und Neubewertungen auch in der Wissenschaft zu gelangen.

Das Sachcomic-Projekt erhofft sich, mithilfe dieses neuen Ansatzes sowohl gesellschaftliche Bedürfnisse als auch kulturell-normative Rahmenbedingungen dialogisch und interkulturell zu integrieren, zu reflektieren und zu hinterfragen. Daraus sollen mögliche Lösungsansätze für die Ernährung der Zukunft destilliert und insbesondere neue Wege der Wissensgenerierung sowie des Wissenstransfers erarbeitet werden. Als Ergebnis dieser partizipativen Forschung wird die Verbindung zwischen Ernährung und persönlichem Ressourcenverbrauch sowohl durch die stilistischen Mittel von Comics als auch durch umfassende Begleitmaterialien verdeutlicht. Dabei sollen nicht nur neue Erkenntnisse und ein Bewusstsein für das Thema Essen generiert, sondern auch ein Kompass für eine Überprüfung und Neubewertung von wissenschaftlichem und gesellschaftlichem Wissen geschaffen werden.

Co-Design: Um die notwendige Transdisziplinarität, insbesondere die Einbindung der Gesellschaft, und hierbei das wechselweise Verschmelzen von Wissensgenerierung und -transfer, zu erreichen, muss die Entwicklung des Storyboards relativ offen bleiben. Die Bildsprache erscheint uns hierbei als das geeignete Medium, wobei wir strukturell folgende Rahmenbedingungen vorgeben:

Das Thema Ernährung soll anhand der drei Eckpunkte 1) Stoffflüsse (lokal, regional, global), 2) Infrastrukturen (Transportwege, Märkte, Haus und insbesondere Küche) und 3) kultureller 
Kontext in möglichst hoher Diversität diskutiert und auf potenzielle, gegebenenfalls ausbaufähige Zukunftsoptionen getestet werden.

Dies wird anhand einer Reise durch zehn Länder in fünf Kontinenten dargestellt. Hierbei werden die drei Eckpunkte nicht standardmäßig nacheinander abgearbeitet, sondern dem gemeinsam mit den Protagonist_innen erarbeiteten Storyboard angepasst, denn viele Aspekte kristallisieren sich erst nach intensivem Austausch heraus.

Diese Offenheit bedingt, dass die einzelnen Geschichten nicht vorab durchgeplant werden können - dies würde eine zu einseitige (westlich dominierte) Sicht auf die Welt generieren. Wir lassen also bewusst möglichst große Freiheiten bei der Entstehung der Narrative zu. Dennoch gibt es Vorgaben, die bei den einzelnen Geschichten entweder zentral (das heißt mithilfe der Protagonist_innen) oder doch zumindest am Rande - in geeigneter Kontextualisierung - eingebaut werden. In diesem Sinne wurde das Element Phosphor als unverwechselbare Hauptfigur eingeführt. Wegen seiner zunehmend kritisch einzuschätzenden Verfügbarkeit ${ }^{22}$ sowie seiner essenziellen Bedeutung für das Leben dient uns dieses Element in personifizierter Form als roter Faden.

Rahmenhandlung: Die bislang geplanten Storyboard-Entwürfe umfassen unter anderem: die Herkunft des Phosphors im kosmischen, erdgeschichtlichen, biologischen und kulturellen Kontext (Prolog); Beispiele zu kompletter Suffizienzwirtschaft (Uganda); geopolitische und soziale Abhängigkeiten industriellen Phosphatabbaus sowie die Wiederentdeckung traditionellen Landbaus unter wissenschaftlichen Aspekten (Marokko); industrielle landwirtschaftliche Massenproduktion und düngemittelbasierte Nebeneffekte, aber auch historische Abrisse der Ernährungsproblematik und deren Lösungsansätze (China); Phosphorrecyling, Überfischung und Plastikverpackungen (Japan); kulturell geprägte vegetarische Ernährung versus gentechnische Verfahren (Indien); soziokulturelle Auswirkungen des Phosphatabbaus auf Banaba (Kiribati/ Mikronesien); Teller-Tank-Problematik und Zuckersucht (Brasilien); moderne Ernährungstrends und -rituale von Öko- bis Kunstnahrung (USA); Fleisch-versus Insektenkonsum (Deutschland). Des Weiteren wird auf die Nahrungsmittelverschwendung und den Umgang mit Müll eingegangen (Norwegen). Aus diesen Themenfeldern werden sich umfassende Reflexionen hinsichtlich der globalen Zukunftsfähigkeit der Ernährung in Form eines interkulturellen Fazits ergeben. Bei allen Kapiteln wird auch das Thema Küche ganz im Sinne des Untertitels des Basisprojektes Die Anthropozän-Küche. Labor der Verknüpfung von Haus und Welt eine besondere Rolle spielen. Die Küche wird hierbei als Trichter des Zusammenfließens der Ressourcen von nah und/oder fern gesehen, als Generator der Nachfrage nach diesen Ressourcen, als strukturelles Labor zur Handhabung, Rekombination und gegebenenfalls Optimierung der Ressourcen sowie als kulturell-soziales Zentrum bzw. Schlüsselfaktor. 
Interkulturelle Partizipation: Neben den Protagonist_innen in den verschiedenen Ländern, mit denen wir die Geschichten entwickeln und personalisieren, arbeiten wir bewusst mit Zeichner_innen aus den jeweiligen Regionen zusammen (Abb.3). Damit soll auch in der bildlichen Gestaltung des Gesamtwerkes den regionalen kulturellen Bedingtheiten Raum gegeben werden. $\mathrm{Ob}$ in der gestalterischen Vielfalt Trends einer globalisierten bildhaften Ausdrucksform sichtbar werden, warten wir mit großem Interesse ab.
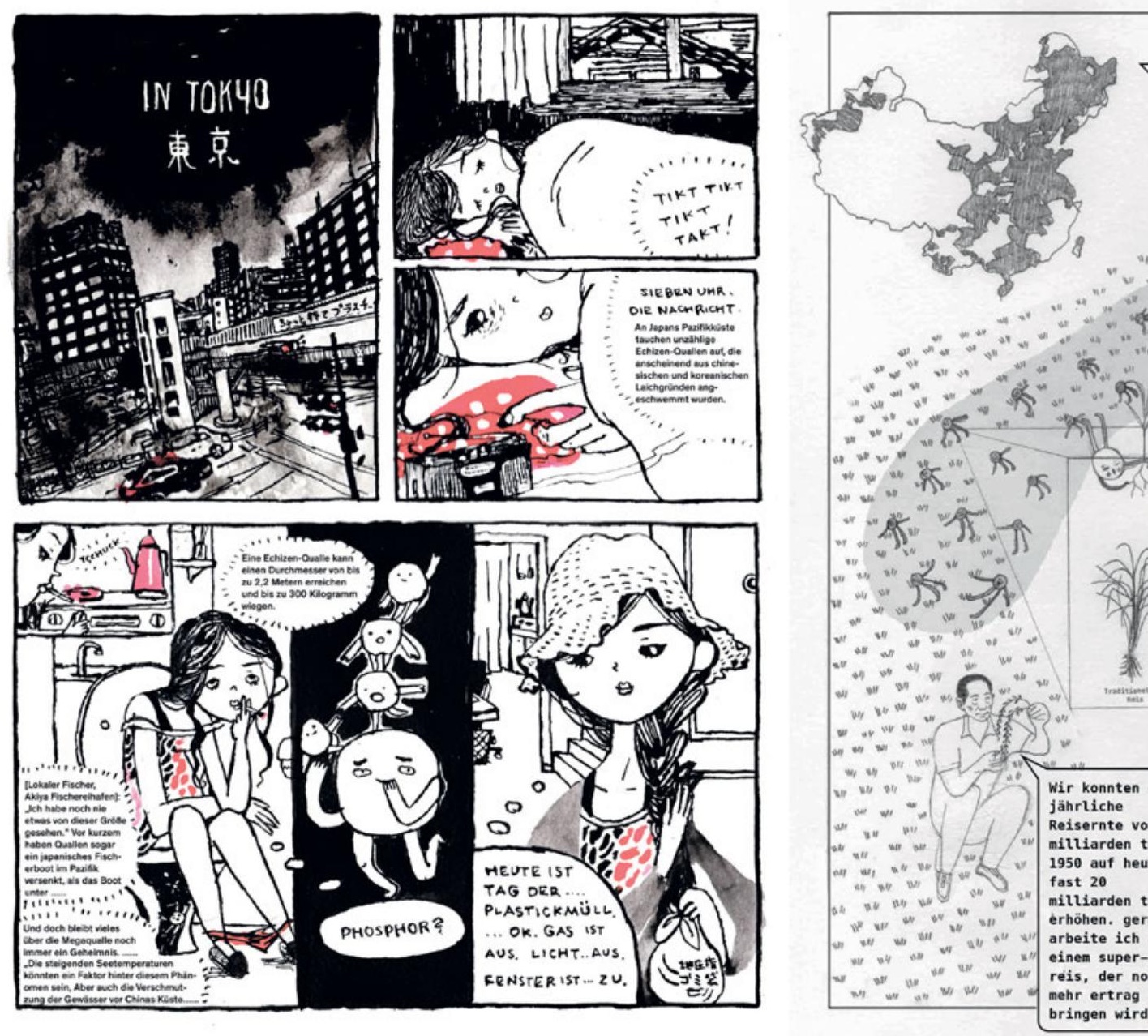

Abb. 3: Von links nach rechts: Skizzen für das Comic-Projekt Die Anthropozän-Küche zu den Kapiteln Japan von Maki Shimizu, China von Ruohan Wang und Brasilien von José Aguiar unterstreichen die künstlerische Vielfalt bei der Umsetzung des Storyboards. 
Kochrezepte als Klammer: Die Frage nach den Lieblings- oder Hauptspeisen unserer Protagonist_innen in den einzelnen Ländern ist jeweils das entscheidende Eingangstor zum Dialog über deren Lebensstil und Essverhalten. Daraus entwickeln wir die Recherchefragen rund um Stoffflüsse, Infrastrukturen und kulturelle Besonderheiten, in deren Zentrum die Betrachtung der jeweiligen Küche als gestaltender, strukturierender und vermittelnder Raum steht. Die Rezepte der Speisen werden bildhaft in ihrer kontextuellen Einbindung im Comic dargestellt, sind nachkochbar und bilden eine Klammer zwischen Protagonist_in und den wissenschaftlichen Themen (Abb.4).

\section{INSTRUCTIONS}
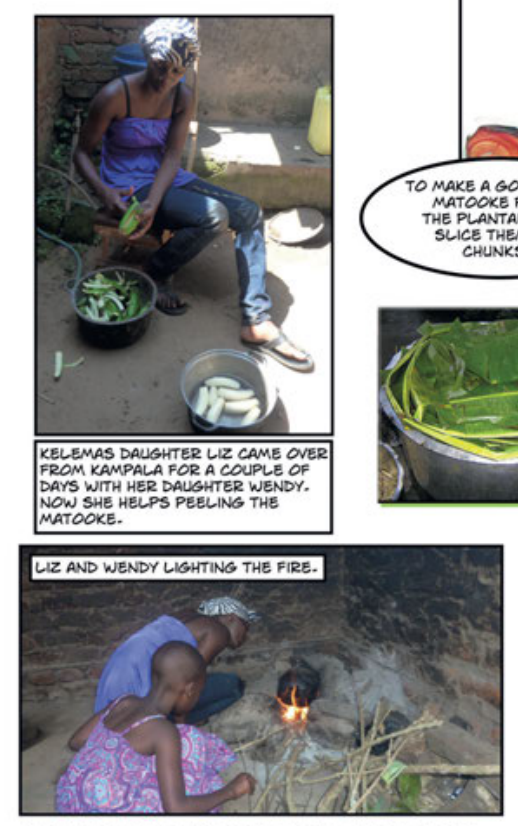

5) SQUEEZE THE MATOOKE WHILE STILL WRAPPED IN THE LEAVES TO MASH THE FRUIT. SERVE IT ON FRESH LEAVES.
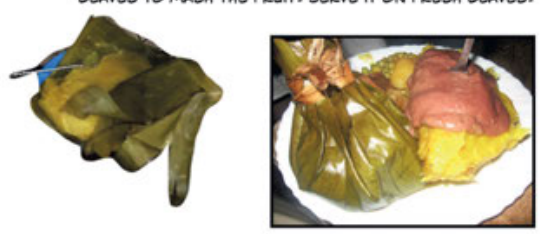
4) PLACE THE POT ON THE
FIRE AND LET THE MATOOKE
STEAM FOR A COUPLE OF STEAM FOR
HOURS.

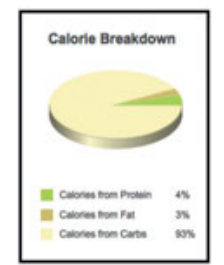

UGANDAN MATOOKE HAS A HIGHCALORIE, VERY HIGH-CARB, PROTEN CONTENT TT IS A GOOD SOURCE OF TPON VITAMIN B6, VITAMIN B12, VITAMIN $C$, NIACIN AND FOLATE.

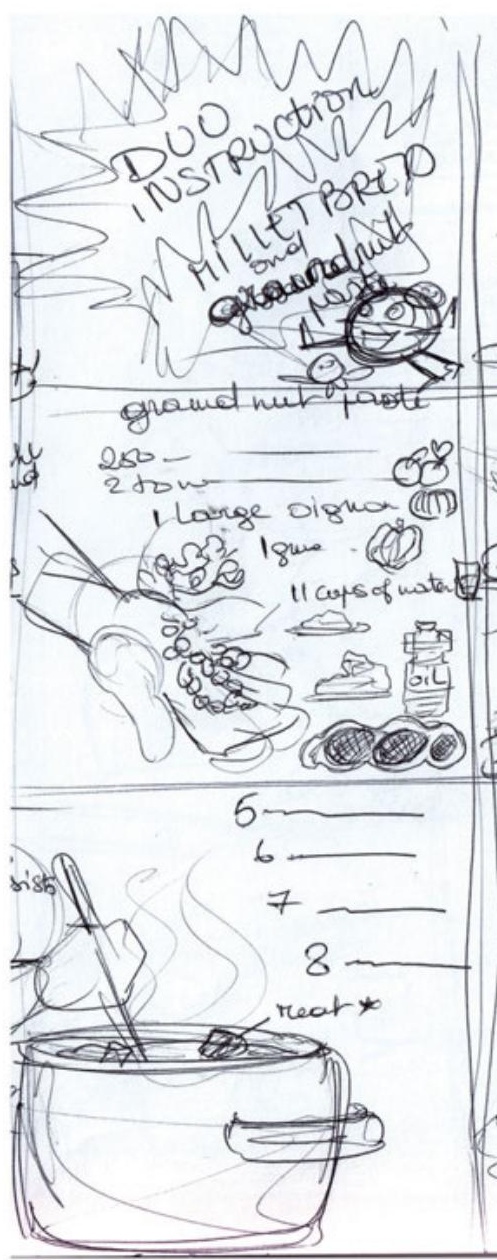

Abb.4: Entwicklungsschritte der Comic-Publikation Die Anthropozän-Küche am Beispiel eines Kochrezepts in Uganda. Von links nach rechts: Auszug aus dem Storyboard von Alexandra Hamann, Skizze und Reinzeichnung von Elyon's. 
Durch diese Herangehensweise können potenzielle Szenarien und Entwicklungspfade für die zukünftige globale Ernährung wissenschaftsbasiert und theoretisch formuliert sowie politisch-gesellschaftlich diskutiert werden. Denkbare Möglichkeiten wären neben einem Weg des business as usual ein Suffizienz-, ein Effizienz-, ein Konsistenz- oder ein Hightech-Pfad. ${ }^{23}$

the rwtet Blecengifos? II water

El carrowar thar $\exists$ anillet flow
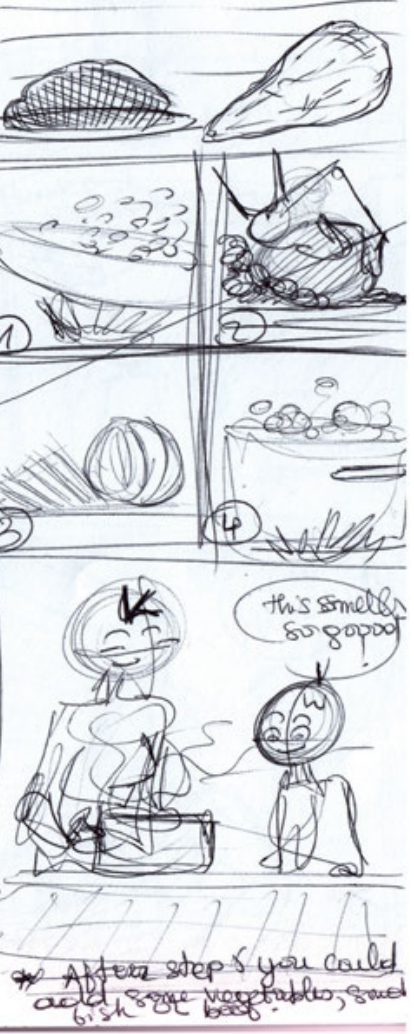
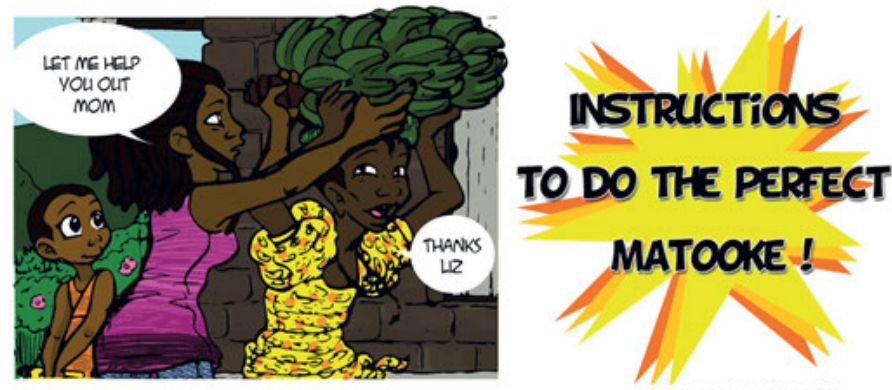

PUT A LITTE WATER IN A COOKNG POT. PLACE WRAPPED

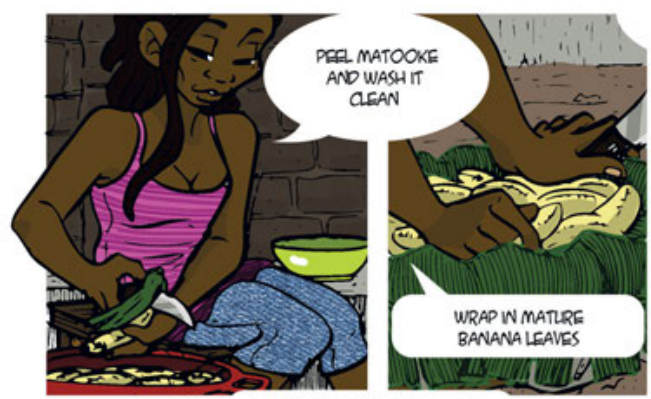

MATOOKE ON TOR

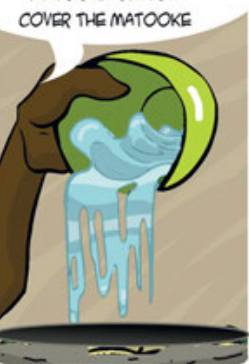

PLACE THE POT ON THE ARE AND LET THE MATOOKE STEAM

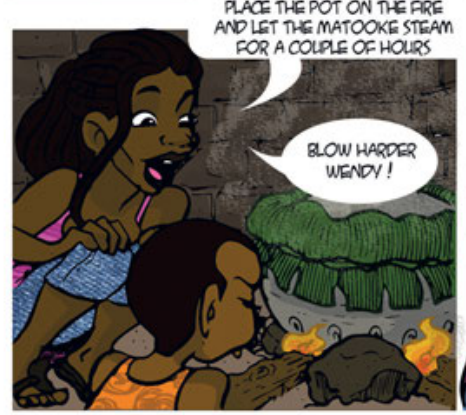

FWALY SQUEECXE THE MATOOKE, WHLE STLL. WRAPPED W THE LEAVES TO MASH THE PRLIT. SERVE IT ON PRESH LEAVES, BEATALL, ISNTIT?

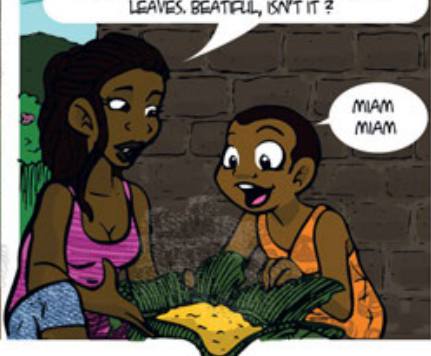




\section{Fazit}

Die kollaborative Entwicklung des Storyboards zwischen Protagonist_innen, Zeichner_innen, lokalen Ansprechpartner_innen und den wissenschaftlichen Mitarbeiter_innen aus dem Projekt stellt eine innovative Form von transformativer Wissenschaft im Sinne eines globalen Realexperiments dar. Wir erhoffen uns, dadurch vielfältige Lösungsansätze für die Zukunft der Ernährung zu identifizieren, die heute tatsächlich gelebt und erprobt werden, aber auch in gesellschaftliche, kulturelle und naturmaßstäbliche Gegebenheiten eingebettet sind. Diese Form der Wissensgenerierung und -reflexion erscheint uns maßgeblich, um auch wissenschaftliche Szenarien verhandelbar und gegebenenfalls umsetzbar zu machen. Zusätzlich wird mit einem Begleitbuch ein klassisches Publikationsformat aufgegriffen, um die aus dem Reallaborprojekt gewonnenen wissenschaftlichen Erkenntnisse zu sammeln und zu dokumentieren. Diese Vertiefungsliteratur soll sowohl für interessierte Leser_innen als auch für Wissenschaftler_innen verschiedenster Disziplinen geeignet sein. 


\section{Literatur}

Brocka, Bruce (1979): Comic Books: In Case You Haven't Noticed, They've Changed. In: Media and Methods, vol. 15, no. 9 , pp. $30-32$

David, Sabria/Blumtritt, Jörg/Köhler, Benedikt (2010): Das Slow Media Manifest. Online unter: http://www.slowmedia.net/manifest (zuletzt aufgerufen: 12. Januar 2015).

Groensteen, Terry (2014): Zwischen Literatur und Kunst. Erzählen im Comic. In: Aus Politik und Zeitgeschichte, Jg. 64, Nr. $33 / 34$, S. $35-42$.

Grünewald, Dietrich (2014): Zur Comicrezeption in Deutschland. In: Aus Politik und Zeitgeschichte, Jg. 64 , Nr. 33/34, S. $42-47$.

Hamann, Alexandra/Feindt, Jan/Leinfelder, Reinhold (2011): Diversity Inaction - Graphic Interview with R. Leinfelder, Cartoon Movement. Online: http://blog. cartoonmovement.com/2011/03/diversity-inaction.html (last access: 12 January 2014).

Hamann, Alexandra/Zea-Schmidt, Claudia/Leinfelder, Reinhold (Hg.) (2013): Die Große Transformation. Klima - Kriegen wir die Kurve? Berlin: Jacoby \& Stuart.

Hamann, Alexandra/Zea-Schmidt, Claudia/Leinfelder, Reinhold (Hg.) (2014): The Great Transformation. Climate - Can We Beat the Heat? Transl. by Culverhouse, Bob. Berlin: WBGU.

Hamann, Alexandra u.a. (Hg.) (2014): Anthropozän. 30 Meilensteine auf dem Weg in ein neues Erdzeitalter. Eine Comic-Anthologie. In Kooperation mit einer Seminarklasse der Universität der Künste Berlin. München: Verlag des Deutschen Museums.

Hangartner, Urs/Keller, Felix/Oechslin, Dorothea (Hg.) (2013): Wissen durch Bilder. Sachcomics als Medien von Bildung und Information. Bielefeld: transcript.

Jacobs, Dale (2007): More than Words: Comics as a Means of Teaching Multiple Literacies. In: The English Journal, vol. 96, no. 3, pp. 19-25.

Jüngst, Heike Elisabeth (2010): Information Comics (Leipziger Studien zur angewandten Linguistik und Translatologie, 7). Leipzig: Verlag Peter Lang.
Leinfelder, Reinhold (2013): Assuming Responsibility for the Anthropocene: Challenges and Opportunities in Education. In: Trischler, Helmuth (ed.): Anthropocene - Envisioning the Future of the Age of Humans (Rachel Carsen Center Perspectives, 2013, 3, pp. 9-18). Munich: Rachel Carsen Center. Online: http://www.carsoncenter.uni-muenchen.de/download/publications/perspec tives/2013_perspectives/1303_anthro_web.pdf (last access: 26 February 2015).

Leinfelder, Reinhold (2014): Das WBGU-Transformations-Gutachten als Wissenschaftscomic: Ein Kommunikationsprojekt zu alternativen Wissenstransferansätzen für komplexe Zukunftsthemen - Ergebnis übersicht. In: SciLogs - Der Anthropozäniker, Spektrum der Wissenschaft. Online unter: http://www.scilogs.de/ der-anthropozaeniker/trafocomicprojekt (zuletzt aufge rufen: 18. Februar 2015)

Leinfelder, Reinhold (2014a): Abschlussbericht zum BMBF-geförderten Projekt »Das WBGU-Transformations-Gutachten als Wissenschaftscomic: Ein Kommunikationsprojekt zu alternativen Wissenstransferansätzen für komplexe Zukunftsthemen«. Projekt-Nr. FKZ OIWJ1213. Unveröffentl. Projektbericht.

Leinfelder, Reinhold (2014b): »Früher war die Zukunft auch besser« - Teil 3: Zukunft? Zukünfte! Zur Notwendigkeit von Zukunftsvisionen. In: SciLogs - Der Anthropozäniker, Spektrum der Wissenschaft. Online unter: http://www.scilogs.de/der-anthropozaeniker/zukunftteil/-zukuenfte (zuletzt aufgerufen: 18. Februar 2015)

Leinfelder, Reinhold (2015): »Die Zukunft war früher auch besser«. Neue Herausforderungen für die Wissenschaft und ihre Kommunikation. In: Möllers, Nina/Schwägerl, Christian (Hg.): Willkommen im Anthropozän - Unsere Verantwortung für die Zukunft der Erde. Ausst.-Kat. Deutsches Museum, München. München: Verlag Deutsches Museum, S. 99-104

McCloud, Scott (1993): Understanding Comics. New York: HarperCollins Publishers.

McCloud, Scott (2014): Was sind Comics? In: Aus Politik und Zeitgeschichte, Jg. 64, Nr.33/34, S. 3-10.

Morrison, Timothy/Bryan, Gregory/Chilcoat, George (2002): Using Student-Generated Comic Books in the Classroom. In: Journal of Adolescent \& Adult Literacy, vol. 45 , no. 8 , pp. $758-767$. 
Plank, Lukas (2013): Gezeichnete Wirklichkeit: ComicJournalismus und journalistische Qualität. Masterarbeit, Fachhochschule Wien. Online unter: https://lukasplank. files.wordpress.com/2013/11/gezeichnete-wirklichkeit.pdf (zuletzt aufgerufen: 18. Februar 2015).

Robin, Libby et al. (2014): Three Galleries of the Anthropocene. The Anthropocene Review. Online: http://anr. sagepub.com/content/early/2014/og/16/2053019614550533 (last access: 18 January 2015).

Schneidewind, Uwe/Singer-Brodowski, Mandy (2013): Transformative Wissenschaft. Klimawandel im deutschen Wissenschafts- und Hochschulsystem. Marburg: Metropolis.

Siggener Kreis (2014): Siggener Aufruf 2014 - Wissenschaftskommunikation gestalten. Online unter: http:// www.wissenschaft-im-dialog.de/wissenschaftskommunikation/weiterentwicklung/siggener-aufruf.html (zuletzt aufgerufen: 18. Februar 2015)

Sousanis, Nick (2015): Unflattening. Cambridge, MA: Harvard University Press.
Vaccari, David A. (2014): Düngemittel: Droht ein Mangel an Phosphor? In: Spektrum der Wissenschaften, Spezial Biologie, Nr.4, S.44-49.

Versaci, Rocco (2001): How Comic Books Can Change the Way Our Students See Literature: One Teacher's Perspective. In: The English Journal, vol. 91, no. 2, pp. 61-67.

WBGU (Hg.) (2011): Welt im Wandel. Gesellschaftsvertrag für eine große Transformation - Hauptgutachten. Berlin: WBGU.

WBGU (Hg.) (2014): Zivilisatorischer Fortschritt innerhalb planetarischer Leitplanken. Ein Beitrag zur SDG-Debatte (Politikpapier, 8). Berlin: WBGU.

Yang, Gene (2008): Graphic Novels in the Classroom. In: Language Arts, vol. 85, no. 3, pp. 185-195. 


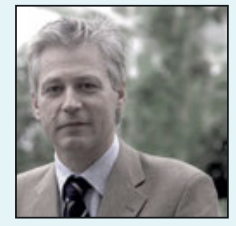

reinhold.leinfelder@fu-berlin.de

Principal Investigator

Basisprojekte: Die Anthropozän-Küche,

Disziplin: Geobiologie

Reinhold Leinfelder ist Geologe und Geobiologe. Neben seiner Forschungstätigkeit bei Bild Wissen Gestaltung ist er Leiter der AG Geobiologie und Anthropozänforschung an der Freien Universität Berlin sowie Mitglied der Anthropocene Working Group der International Stratigraphic Commission. Seit September 2014 ist er Gründungsdirektor des Hauses der Zukunft in Berlin.

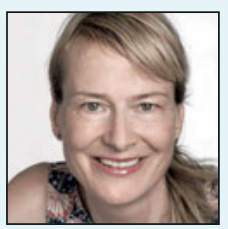

alexandra.hamann@mintsites.de

Freie Mitarbeiterin, Associated Member

Basisprojekt: Die Anthropozän-Küche

Disziplin: Mediendesign

Alexandra Hamann ist Mediendesignerin und leitet seit 2001 eine Agentur für Wissenskommunikation und Bildungsmedien. Seit einigen Jahren beschäftigt sie sich eingehend mit neuen Wegen der Wissensvermittlung. Im Exzellenzcluster verantwortet sie die Erstellung und Umsetzung der Storyboards für die Comic-Publikation Die Anthropozän-Küche, die das Fachwissen der wissenschaftlichen Mitarbeiter_innen des Basisprojekts und das Erfahrungswissen der Protagonist_innen zusammenführen.

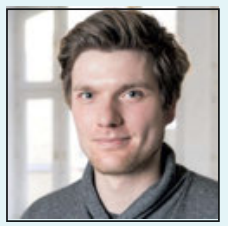

jens.kirstein@fu-berlin.de

Wissenschaftlicher Mitarbeiter

Basisprojekt: Die Anthropozän-Küche

Disziplin: Geologie

Jens Kirstein ist Geologe und wissenschaftlicher Mitarbeiter an der Freien Universität Berlin. Neben seiner Tätigkeit im Basisprojekt Die Anthropozän-Küche am Exzellenzcluster Bild Wissen Gestaltung ist er Mitglied der International Max Planck Research School for Global Biogeochemical Cycles und promoviert an der Friedrich-Schiller-Universität Jena über die quartäre Landschaftsentwicklung des Saaletals hinsichtlich Karbonatverwitterung und Stofftransport. 


\section{Morphologische Analysen vegetabiler Ornamente der Gotik}

\section{Eine interdisziplinäre Annäherung an kunsthistorische Reihen*}

Die vorliegende Untersuchung erläutert anhand gotischer Kriechblumen die Verknüpfung der vergleichenden kunsthistorischen Arbeitsweise mit der morphologisch-phylogenetischen Analyse. 'Das vegetabile Ornament wird dabei auf die innewohnenden strukturellen Eigenschaften hin untersucht und zu Gruppen zusammengefasst. Darüber hinaus zielt die Analyse daraufab, genealogische Momente herauszuarbeiten, die zur Genese einer neuen Reihe und zu einem veränderten Modus in der Gestaltung führen. Analog zu biologischen Verwandtschaftsbeziehungen können mithilfe des phylogenetischen Verfahrens diese Entwicklungslinien und Abhängigkeiten innerhalb einer untersuchten Objektgruppe anhand der Ausbildung ausgewählter Eigenschaften sichtbar gemacht werden.

* Kubler 1963, 54, definiert die Reihe als Abfolge von Ereignissen (= Schaffung eines neuen Objekts gleicher Formkategorie). Ihnen kann aus der historischen Perspektive kein weiteres Ereignis hinzugefügt werden. In dieser Reihe verringert jede Positionsbesetzung die Anzahl weiterer Positionen, zudem sind die weiteren Handlungsmöglichkeiten eingeschränkt: »Every new form limits the succeeding innovations in the same series.«

1 Die Arbeit versteht sich auch als eine Kritik an der älteren kunsthistorischen Forschung, deren Fokus auf der Ableitung vegetabilen Bauschmucks aus real existierenden Pflanzengattungen lag. Vgl. Roth 1976. Ansätze dazu bereits bei Riegl 1893, insbes. Kapitel III. 


\section{Die kunsthistorische Fragestellung}

Ausgangspunkt dieser Untersuchung ist die Frage nach den Entwurfsprozessen und Mechanismen der Formwerdung und des Formwandels vegetabiler Ornamente der mittelalterlichen Kunst. Als Untersuchungsgegenstände aus den Leitmotiven gotischer Kunstproduktion bieten sich die bekrönenden Architekturelemente mit Wimperg und Fiale an. Wesentliche Bestandteile dieses Giebelmotivs sind »Laubpossen «, bestehend aus den Krabben, auch Kriechblumen genannt, und der »recht plum auf dem Wimperg «, die als Kreuzblume den Giebelscheitel bekrönt (Abb. 1). Beide zeitgenössische Termini »Laubposse « und »plum « sind spätgotischen Lehrwerken zur architektonischen Entwurfstechnik von Matthäus Roriczer entnommen. ${ }^{2}$ Die eng miteinander verbundenen Ziermotive wurden über die Architektur hinaus in weiteren Medien der bildenden Kunst verwendet. Die geschnitzten Possen an den rückwärtigen Tafeln und an den Hochwangenabschlüssen der Chorgestühle stehen im Zentrum dieser Untersuchung.

Es lag nahe, Prinzipien der Formwerdung in den Lehrwerken zu suchen, da sie als Wissensspeicher existierender Praktiken verstanden werden können. Andererseits vermögen sie auch Hinweise über den Formenwandel zu geben, zumal im Veröffentlichungszeitraum der Roriczer-Schriften eine Veränderung im Ornamentsystem stattfand, im Zuge derer vegetabile Elemente, insbesondere das Astwerk, gegenüber Maßwerkverzierungen eine neue Gewichtung erlangten. ${ }^{3}$ Möglicherweise kam den Kriechblumen während dieses Ornamentwechsels, sicher aber in der Ausbildung der Steinmetzen, eine gewichtige Rolle zu, wie ihr zahlreiches Vorkommen in den Musterbüchern und der Druckgrafik belegt. ${ }^{4}$

Die Konstruktion der architektonischen Formen macht Roriczer in aller Ausführlichkeit mithilfe mathematisch-geometrischer Berechnungen und Anweisungen zur Nutzung des Zirkels nachvollziehbar, wobei jedoch ein Widerspruch zwischen der Aktualität der vermittelten mathematisch-geometrischen Praxis und den dargestellten künstlerischen Formen offenbar wird. ${ }^{5}$

2 Zit. aus Geldner 1965, 46, mit Textübertr. und Faksimile der Schriften puechlen der fialen gerechtikait von 1486 und Geometria Deutsch von 1486/87. Die adjektivische Erläuterung »recht plum « legt den Blattentwurf aus einer rechtwinkligen Grundform nahe. Zur Vereinheitlichung der Terminologie wird nachfolgend der Terminus Kriechblume anstatt Laubposse oder Krabbe und Kreuzblume anstatt »plum «verwendet. Posse (auch Bosse) bezeichnet im Mittelalter jedes gehauene Bildwerk, hier also das Laubwerk. Im 15. Jahrhundert setzt sich ausgehend vom italischen Raum die Bedeutung als Rohfassung eines Werkstückes durch, sodass nicht sicher gesagt werden kann, ob Roriczer auf die ausformulierte Form oder die Rohform abzielt. Vgl. Arens 1948.

3 Eine Darstellung der Genese des Astwerkstils kann hier nicht erfolgen, als Ausgangspunkt für dessen Entwicklung werden jedoch veränderte Darstellungen der Kriechblumen an Kapitellen sowie an Chorgestühlen herangezogen. Vgl. Lemper 1950, $18 \mathrm{f}$.

4 Insgesamt 31 Krabben enthält das als Lehrlingszeichnungen interpretierte Laubhauerbuch des Matthäus Böblinger von 1435, siehe Bucher 1979. Vgl. auch die Krabben im Wiener Musterbuch (Rathe 1926) und die um 1465 entstandenen Kupferstiche von Schongauer. Nach der Handwerksordnung der Maurer und Steinmetzen im Amt und Stadtgericht Querfurt vom Jahre 1574 (abgedruckt in Wissell 1986, 419, Art. XXXVII) mussten die Steinmetzgesellen noch im 16. Jahrhundert eine zwei Jahre dauernde Zusatzausbildung absolvieren zur künstlerischen Spezialisierung auf Laubwerk, Bildnisse und dergleichen.

5 Vgl. Geldner 1965, 73, zur mathematischen Praxis. Roriczers künstlerische Formen folgen nicht den jüngsten Gestaltungsprinzipien, zu denen das Übereinanderlegen unterschiedlich rhythmisierter Gliederungen in verschiedenen Raumund Wandschichten sowie Torsionen der Architekturglieder zählen. 


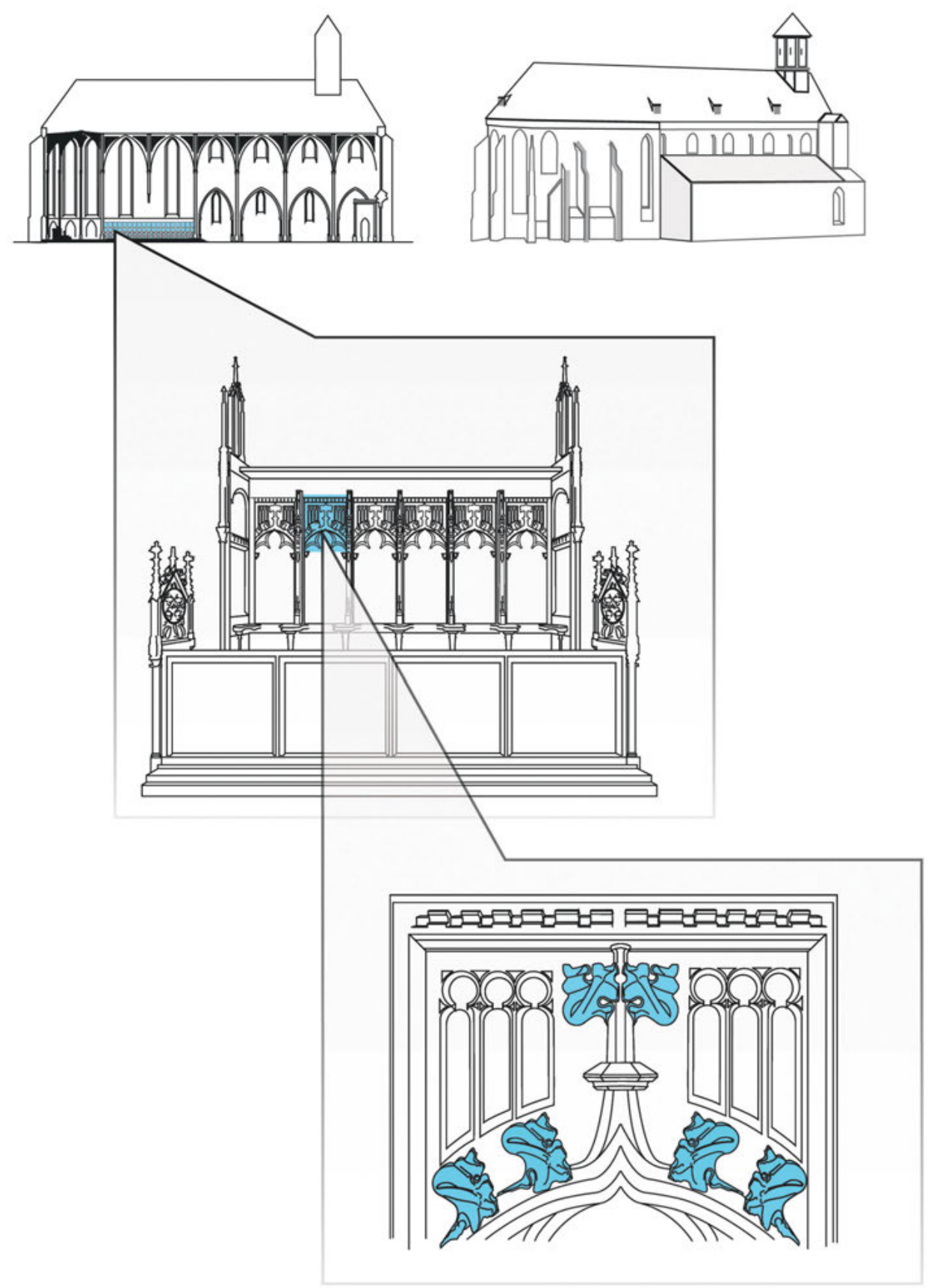

Abb. 1: Verortung der Chorgestühle im Kirchenraum und der Kriechblumen am Chorgestühl. 


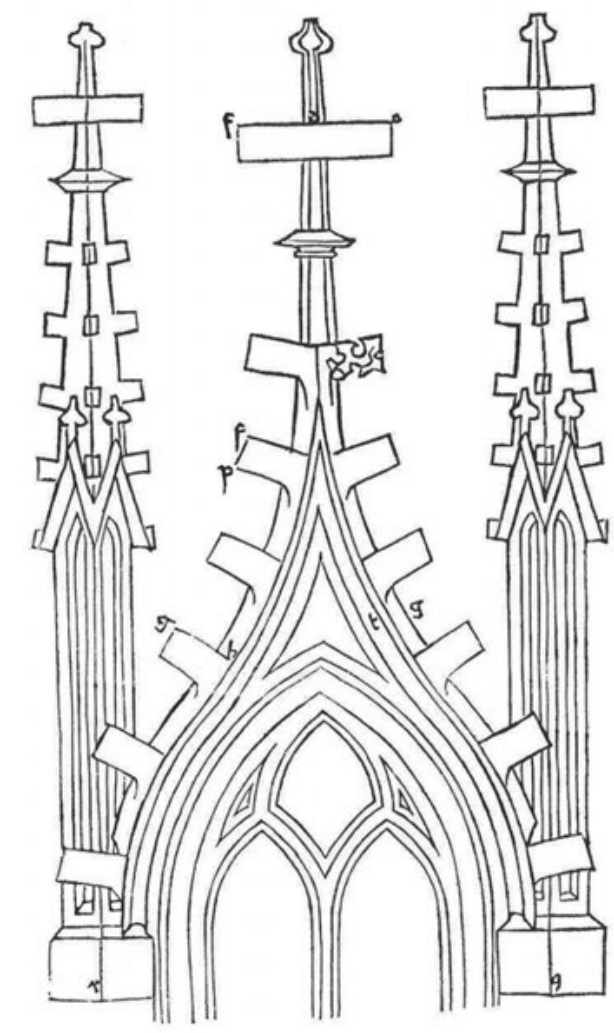

Abb. 2: Wimpergkonstruktion mit Kriechblumenschemata nach Matthäus Roriczer (1487/88), Geometria Deutsch, Regensburg, Bl. 6v.

Zudem beschränken sich die Erläuterungen zum Entwurf pflanzlicher Komponenten im Ornamentsystem auf die Positionierung und Proportionierung der Blattwerkgrundfiguren, die zumeist durch quadratische oder rechteckige Platzhalter angedeutet werden (Abb. 2). Nur vereinzelt gibt er in den beigegebenen Zeichnungen das vegetabile Aussehen der Kriechblumen mittels Umrisslinien wieder. Angesichts einer fehlenden Dokumentation des künstlerischen Prozesses und der Entscheidungen, anhand derer nachvollziehbar wäre, wie aus den geometrischen Grundformen vegetabile Formen entstehen, kann einerseits davon ausgegangen werden, dass Roriczer mit seinen Schriften ausschließlich die Vermittlung der geometrischen Konstruktion intendierte. Darüber hinaus ist es aber möglich, dass Kriechblumen zum routinierten Wissen und Können der Baumeister und Schnitzer gehörten, sodass die Notwendigkeit, ihre Formwerdung explizit zu machen, nicht bestand. 
An dieser Stelle setzt das morphologische Experiment an, das Methoden entwickeln möchte, um die Entstehung artifizieller Formen und künstlerische Prozesse abzubilden. Als Untersuchungsgruppe wurde eine in sich geschlossene Werkgruppe ausgewählt, deren Entstehungszeitraum deutlich vor dem angedeuteten Ornamentwechsel liegt, sodass die Voraussetzung für eine objektive Vergleichbarkeit und eine sichere Basis für die spätere Ausweitung der Untersuchungszeiträume gegeben sind. Die Werkgruppe altmärkischer Chorgestühle bietet mit sehr markanten, an allen Gestühlen wiederkehrenden Detailformen eine breite Basis an Analyseobjekten. Die beiden umfangreichen Chorgestühle der Nikolaikirche und der Jacobikirche in Stendal wurden zwischen 1430 und 1440 geschaffen. Die Formensprache der Stendaler Stücke ist bereits in einem Gestühl aus der Zeit um 1390 in der Salzwedeler Marienkirche angelegt, das in unserem Experiment als Außengruppe dient. ${ }^{6}$

Unser Experiment stellte unter anderem folgende Fragen an die Kriechblumen:

> Wie lassen sich Formen derart beschreiben, dass sie anhand verschiedener Beispiele verglichen werden können?

> Welche markanten Einzelteile enthalten sie?

> Welche Formaspekte sind konstant, welche variabel?

> Unterliegen beobachtete Variationen einem Zufallsprinzip?

> Resultieren Differenzen aus einer handwerklich geprägten Gestaltungspraxis, und markieren diese zugleich eine Hierarchisierung im Ornamentsystem?

Darüber hinaus kamen weitergehende, vor allem kunsthistorisch orientierte Fragen auf:

> Haben das Fehlen oder Vorhandensein einzelner Merkmale bzw. eine veränderte >Wuchsrichtung< Einfluss auf die Wirkung des Ornamentsystems?

> Welche Hinweise auf handwerkliche Prozesse lassen sich an der Form ablesen und wie können diese in einen genetischen Ablauf eingegliedert werden (Bauphasenplan)?

> Decken sich unsere Beobachtungen mit den Beschreibungen zur Konstruktion von Kriechblumen (Roriczer), und welche Erkenntnisse gewinnen wir darüber hinaus?

6 Zur Einordnung der Gestühle siehe Sachs 1960. 


\section{Die interdisziplinäre Methodik}

Nachdem die Objekte ausgewählt waren, dokumentierten Mitglieder des Basisprojektes Genese und Genealogie der Form die Kriechblumen vor Ort. Anschließend wurden die Merkmale konzeptualisiert, in einer Tabelle gelistet und durch mehrmaligen Abgleich mit den Objekten konkretisiert. Dies erfolgte in enger Zusammenarbeit zwischen Kunstgeschichte und Biologie. Dabei wurden zunächst alle Merkmale gleichberechtigt behandelt und erst bei der Auswertung neu kategorisiert. Für jedes Merkmal wurden die möglichen Ausprägungsformen (Merkmalszustände) klar definiert und zur besseren Nachvollziehbarkeit in schematischen Grafiken fixiert (Abb.3). Stellvertretend sei der Rücken genannt, der als Merkmalszustand entweder vorhanden ist oder nicht; ist er erkennbar, ist er gerundet bis stark bucklig aus der Kriechblumenfigur herausgearbeitet.

Aus dieser Tabelle wurden eine Datenmatrix erstellt und ein Stammbaum mit einer phylogenetischen Software berechnet (Abb. 4).? Zur Bestimmung der Leserichtung, sprich der zeitlichen Dimension, wird der Stammbaum an einem in seinen Merkmalen als ursprünglich angenommenen Merkmalsträger (Außengruppe) gewurzelt. In unserem Experiment setzten wir die Wurzel an die früher gefertigten Kriechblumen der Marienkirche in Salzwedel. Aufgrund der Merkmalsvarianten werden die historischen Formveränderungen in abgestuften Verwandtschaftsverhältnissen in Form eines Kladogramms dargestellt. ${ }^{8}$ Grundlegend für die Interpretation der Stammbäume ist, dass es sich nicht um einen einseitig gerichteten Graphen handelt, das heißt, dass alle Verzweigungspunkte drehbar sind. An den Verwandtschaftsverhältnissen ändert sich indes nichts. Der verwendeten Methode der Stammbaumberechnung liegt das Sparsamkeitsprinzip zugrunde: Der Stammbaum mit der geringstmöglichen Anzahl von Transformationsschritten wird favorisiert. In der Kunstgeschichte wie auch der Biologie ist diese Sparsamkeit natürlich nicht immer gegeben; vielmehr müssen wir die Möglichkeit einer gelegentlich forcierten Formänderung in Erwägung ziehen.

Das Basiskladogramm zeigt den grundlegenden Verlauf der Formänderung anhand aller eingespeisten Daten (Abb.4). Die erste Aufspaltung bildet die Veränderung zwischen der Salzwedeler Kriechblume und den Stendaler Formen ab. Zur besseren Sichtbarkeit der Merkmalszustände werden die Knoten in den Kladogrammen bei der Ansicht der Einzelmerkmale farbkodiert wiedergegeben. Der zweite Knoten zeigt, dass die Stendaler Formen der Nikolaikirche und der Jacobikirche jeweils eine distinkte Gruppe bilden, obwohl einzelne Kriechblumen beider Kirchen auf den ersten Blick Gemeinsamkeiten aufweisen. Die Zusammengehörigkeit der Kriechblumen der Jacobikirche beispielsweise beruht unter anderem auf dem neuen Merkmalszustand eines kurzen Stiels, der unmittelbar an bzw. selten etwas hinter der Umrisslinie der Blattkrabben endet. Ursprünglich ragt der Stiel weit über die quadratische Grundfigur der Kriechblume hinaus - ein Merkmalszustand, wie er in der Nikolaikirche und in der Salzwedeler Marienkirche auftritt. St. Nikolai ist durch die Merkmalszustände eines gemuldeten und des unregelmäßig

7 Goloboff/Ferris/Nixon 2000; Maddison/Maddison 2014.

8 Siehe Scholtz 2013, 38 . 


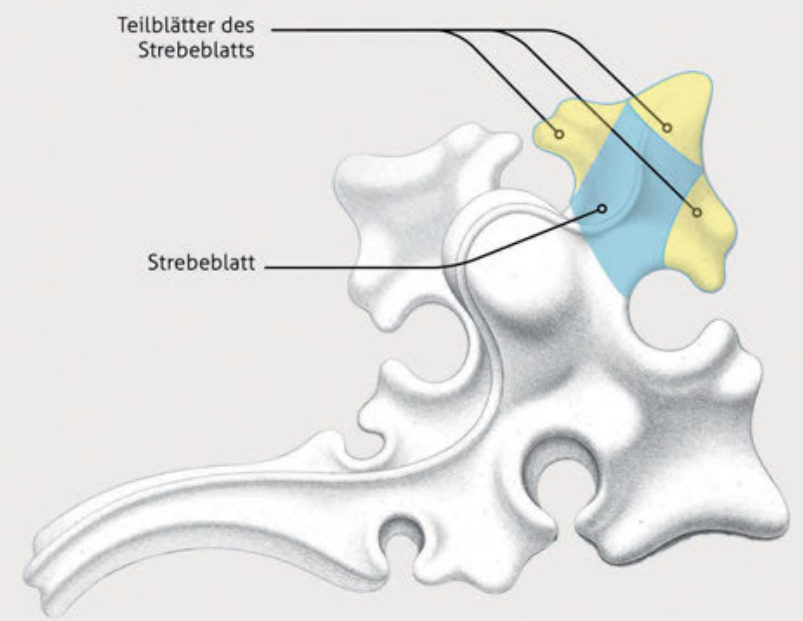

1 spitz

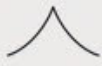

2 spitz gestutzt

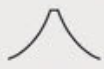

3 gerundet

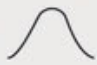

4 unregelmäßig

gestutzt

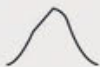

6 gedellt
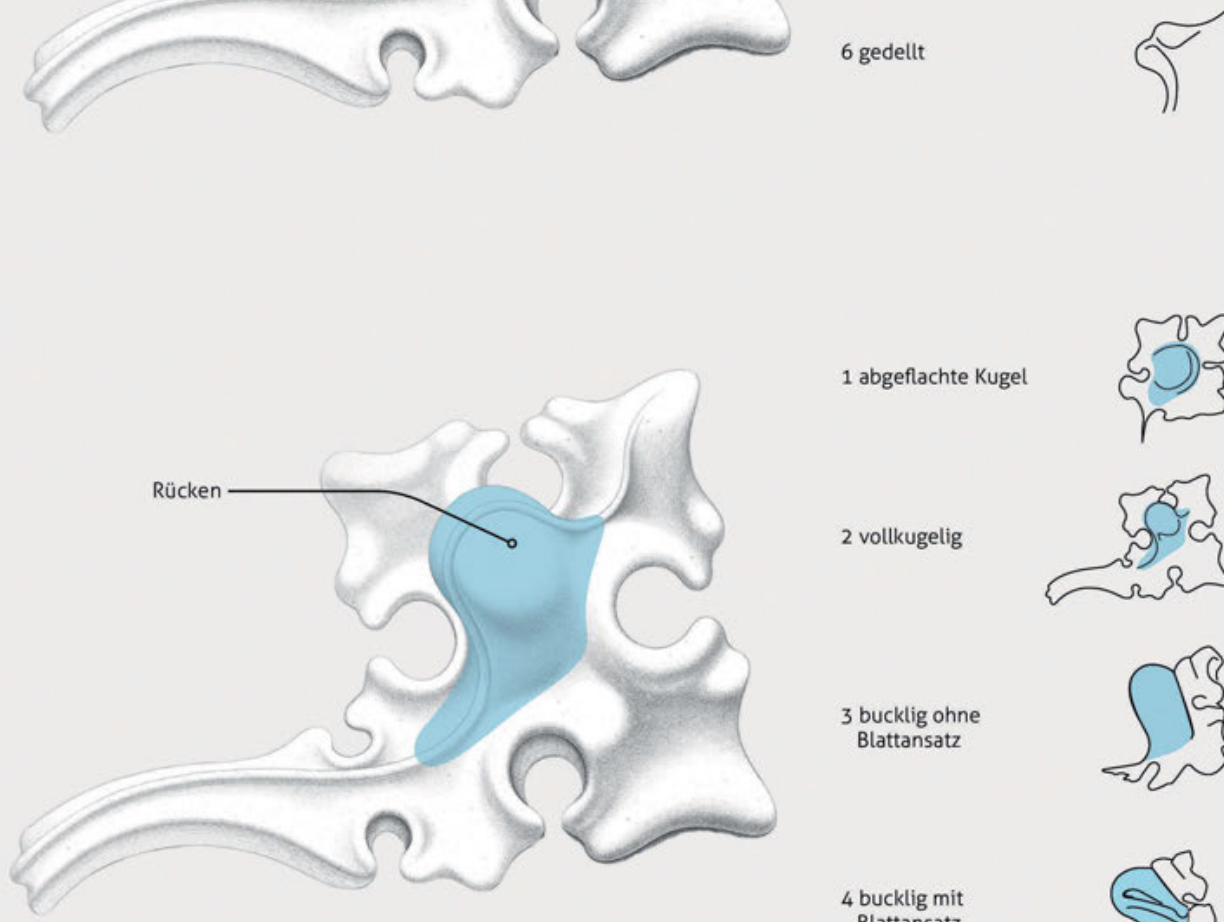

1 abgeflachte Kugel

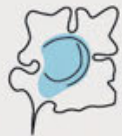

2 vollkugelig

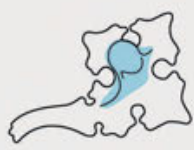

3 bucklig ohne

Blattansatz

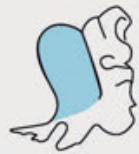

4 bucklig mit

Blattansatz

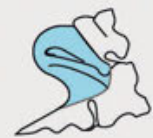

Abb. 3: Exemplarisches Schema der Kriechblumen mit Merkmal und Merkmalszustand, Beispiel Idealtyp

19. Jahrhundert, Merkmale: Form der Teilblätter (oben) und Rücken (unten). 


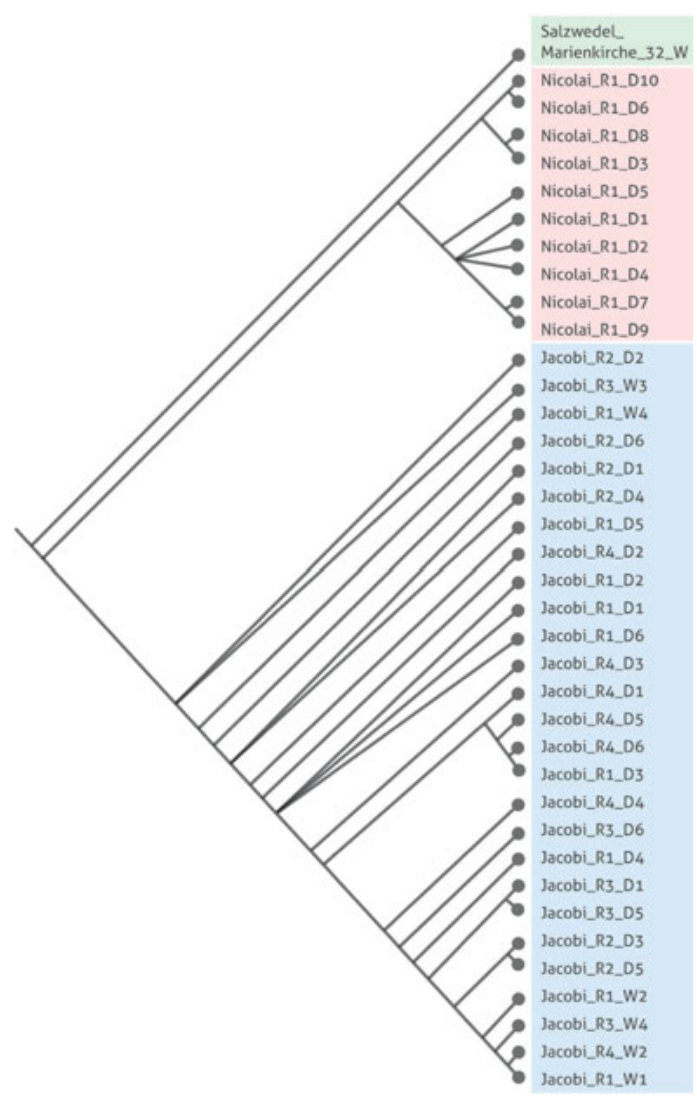

Abb. 4: Basiskladogramm. Erstellt mittels Tree Analysis Using New Technology (TNT).

runden Halteblattes charakterisiert (Abb. 5). Diese beiden Merkmale können in St. Jacobi und St. Marien nur vereinzelt beobachtet werden.

Bezogen auf das Rückenmerkmal zeigt sich eine Bevorzugung jeweils zweier Rückenvarianten an einem Ort: > bucklig ohne Blattansatz< in Nikolai und Jacobi, > bucklig mit Blattansatz< nur in Jacobi bzw. >spitz mit Blattansatz< nur in Nikolai. Zudem zeichnet sich bei den Kreuzblumen eine deutliche Tendenz zur buckligen Rückenform mit Blattansatz ab, besonders gehäuft an den Gestühlen der Jacobikirche und bei etwa jeder zweiten Kreuzblume jener in St. Nikolai. Die Nikolaigruppe spaltet sich in zwei Untergruppen auf, deren Vertreter bis auf wenige Ausreißer alternierend angeordnet sind. Die allgemeine Formvarianz in diesem wie auch in anderen Merkmalen ist in Nikolai sehr gering; die Gestühle dort zeichnen sich also durch eine sehr homogene Gestaltung aus. 


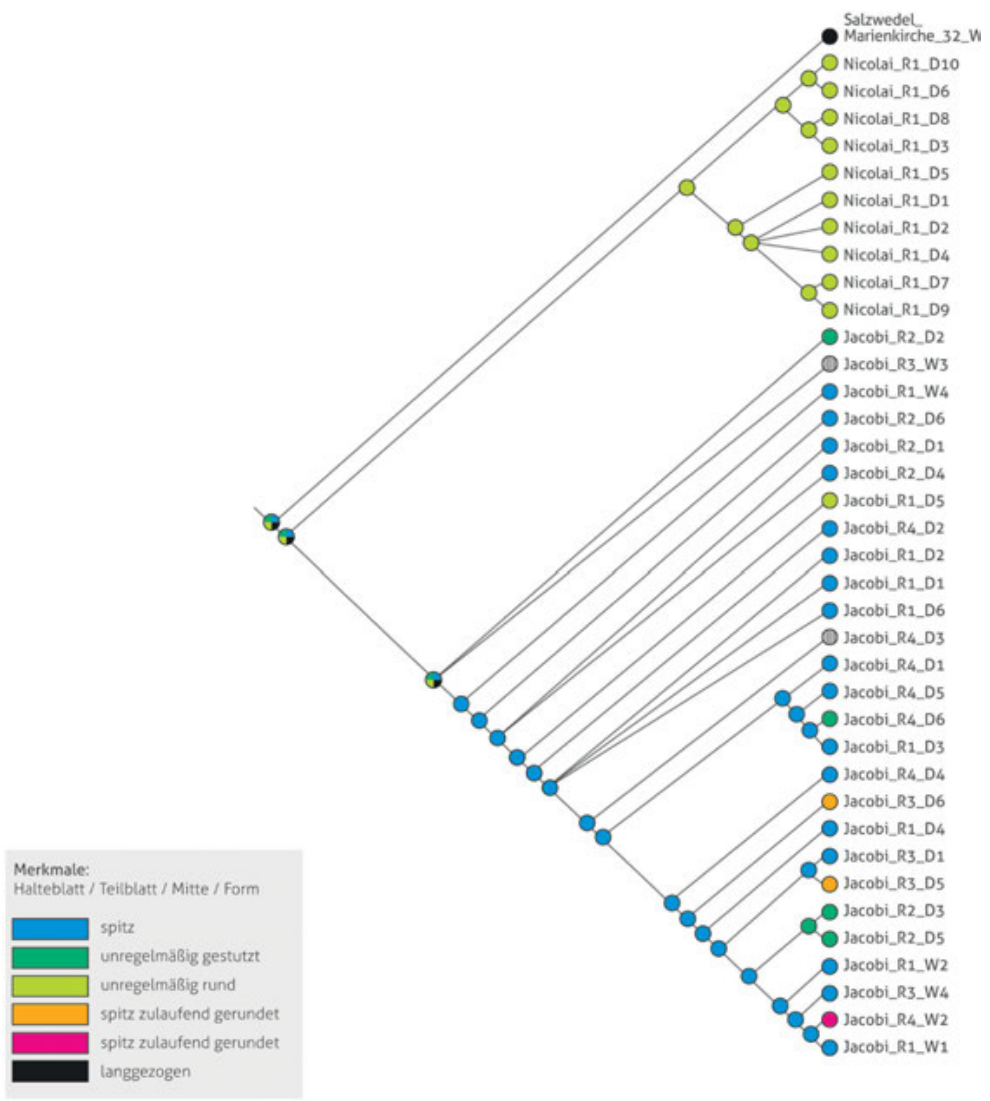

Abb. 5: Kladogramm mit der Verteilung der Merkmalszustände in den Teilblättern.

Im Vergleich dazu zeigen die Jacobigestühle eine sehr breite Varianz, wie das heterogene Farbenspektrum der Jacobiknoten recht gut veranschaulicht (Abb. 5). Das hier herausgezogene Merkmal bezieht sich auf die Gestaltung der Blätter, die wir in mehrere Teilblätter unterteilt haben. Die Form der Teilblätter wird durch die Zustände >spitz<, > gestutzt<, > unregelmäßig gestutzt< bzw. >gerundet< etc. beschrieben. In Nikolai ist ausschließlich der Merkmalszustand unregelmäßig gerundet nachzuweisen. In Jacobi hingegen findet sich nicht nur an nahezu jeder Kreuzblume ein anderer Merkmalszustand, sondern auch innerhalb eines Blattes differiert die Form stark. Es würde den Rahmen dieses Beitrages sprengen, die Differenzen und Abhängigkeiten aller Merkmale zu erläutern. Vielmehr soll abschließend auf unsere kunsthistorischen Kernfragen nach der handwerklichen und/oder künstlerischen Formgenese eingegangen werden. 


\section{Nutzbarmachung der Ergebnisse für die kunsthistorische Forschung}

Die Interpretation der vorgestellten Daten steht in einem engen Zusammenhang mit Varianz und Entwurfspraxis sowie insbesondere mit der handwerklichen Umsetzung. Dieser Gedankengang sei exemplarisch erläutert anhand zweier Kriechblumen, je einer aus St. Jacobi und St. Nikolai (Abb. 6, Abb. 7 rechts). Beide Beispiele gehören zu den Dreiblatttypen. Der Rücken ist bei dieser Variante stark verschliffen, die Blattansätze sind bis weit an den Stiel heruntergezogen. Die Version von St. Nikolai weist eine aufwendige Detailgestaltung auf, mit Blattadern und bewegt gewellten Teilblättern sowie individuell mehr oder weniger tief gerundeten Buchten zwischen denselben. In Jacobi dagegen weist der gleiche Grundtyp eine glatte Oberfläche auf, Adern fehlen, und die Buchtungen der Teilblätter sind durch flache, aber scharf einschneidende Kerben markiert. Für die Blattformen mussten wir besonders häufig den Merkmalszustand unregelmäßig gestutzt vergeben.

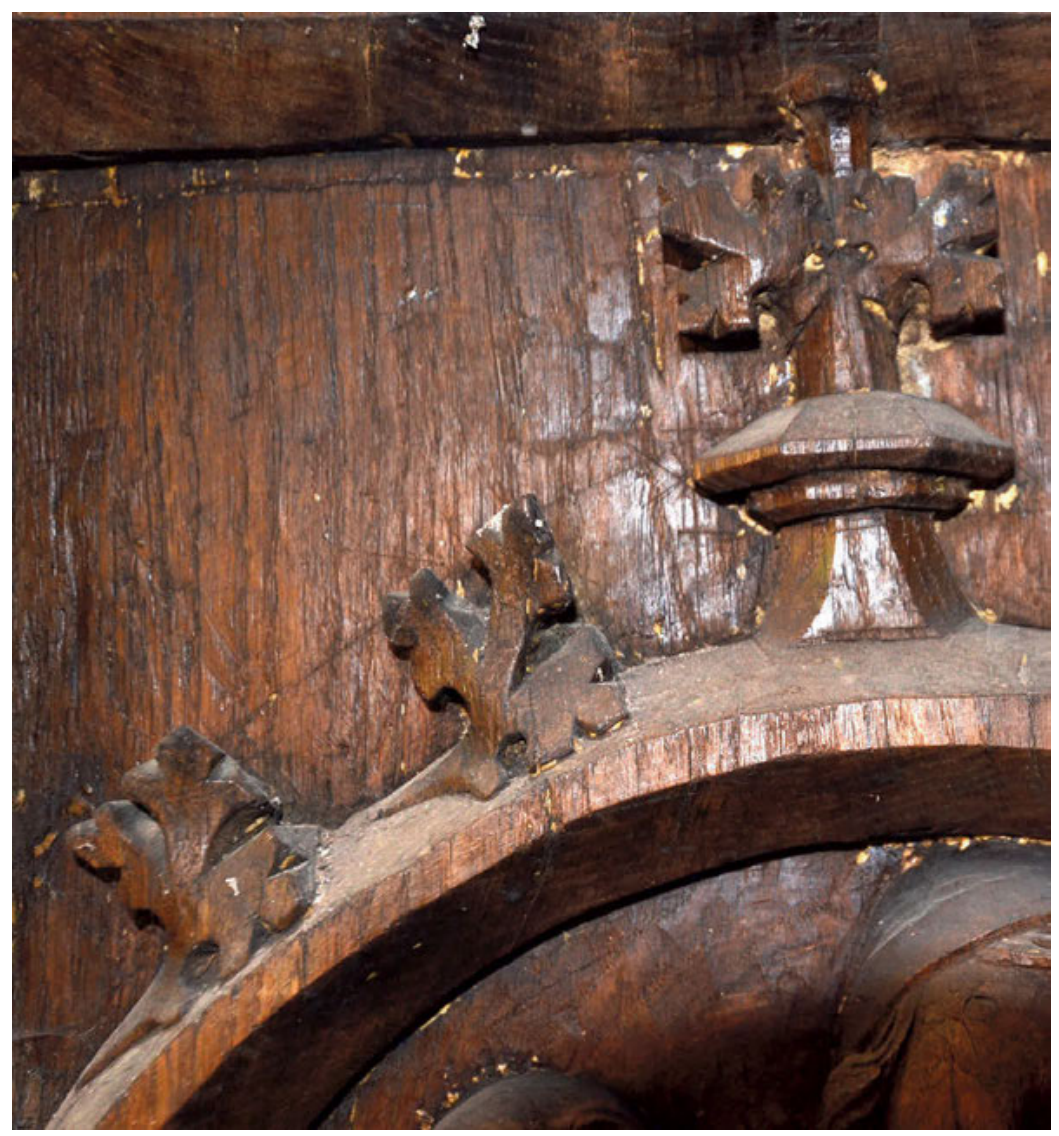

Abb. 6: Schablonenhaft bearbeitete Kriechblumen an den Wangen der Jacobikirche in Stendal, Detail, um 1440 . 


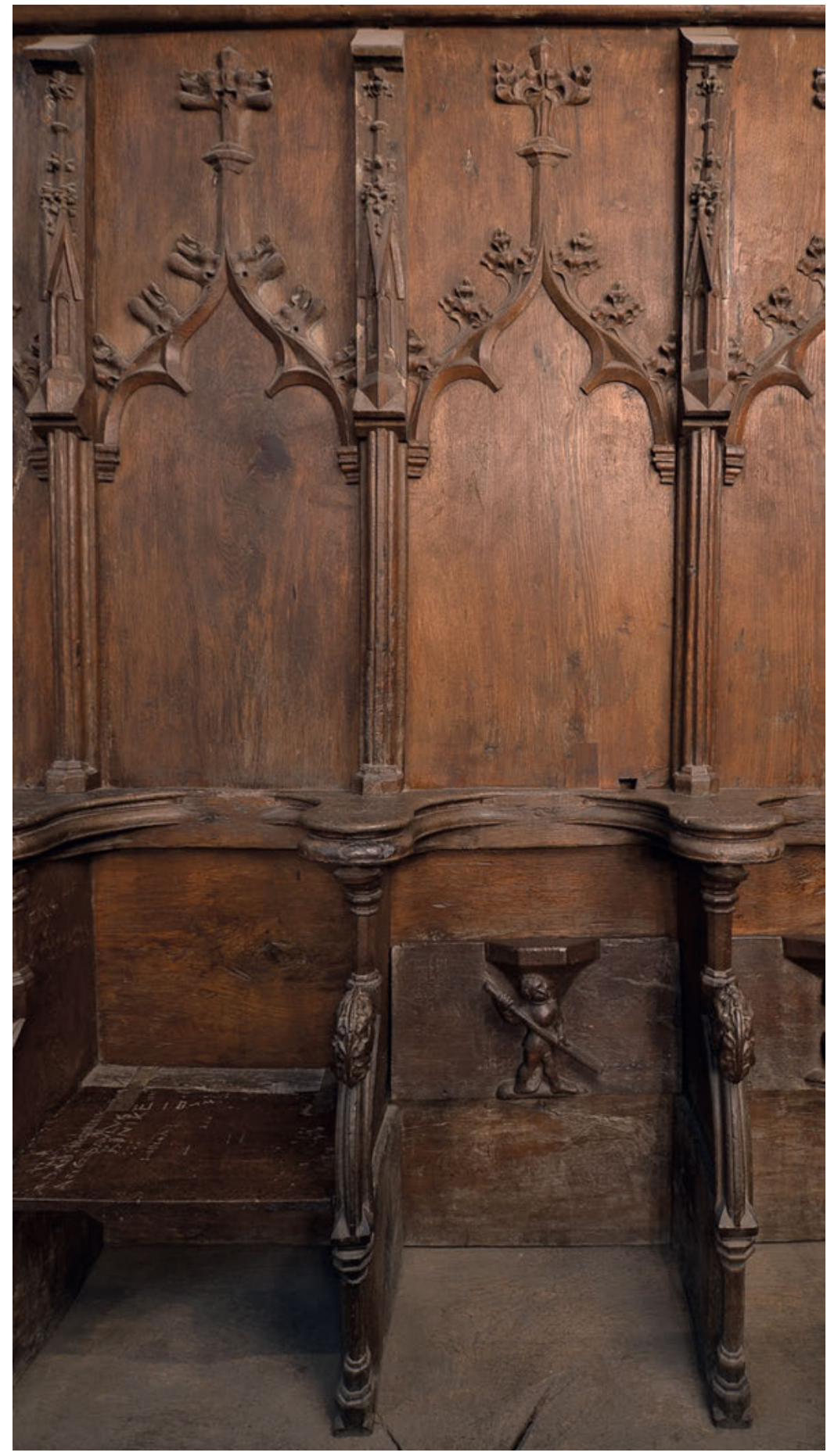

Abb. 7: Rechts Wimperg mit Kriechblumen vom Dreiblatttyp am Bogen und Zweiblatttyp als Kreuzblume, links Dorsalfeld mit ausschließlicher Verwendung des schneckenförmigen Zweiblatttyps, um 1430, Nikolaikirche in Stendal. 
Die Gründe hierfür sind im handwerklichen Prozess zu suchen, da in einem sehr frühen Stadium zu entscheiden war, welche Gestalt die Blattkrabben erhalten sollten. Die quadratische Umrisslinie, wie man sie aus den Werkmeisterbüchern kennt, ist an beiden Grundfiguren ablesbar, auch wenn es sich in diesem speziellen Fall um Abdrücke der Stechbeitel beim Abstechen entlang der Kontur handelt. In Nikolai wurden diese Bearbeitungsspuren gründlich entfernt. Unter großem Aufwand ausgeführt sind in beiden Varianten die tiefen geschwungenen Buchten zwischen den Hauptblättern. Hingegen liegen den unregelmäßigen Formen der Teilblätter die Prinzipien der Einfachheit und Schnelligkeit zugrunde: Die Herstellung eines Teilblattes aus einer eckigen Grundform lässt sich rasch durch das Abstechen der scharfen Ecken und das winklig angesetzte Einschneiden der Kerben erreichen. Ebenso konnte die glatte Oberfläche durch gekonnte Hobelstöße angelegt werden. Dieser schlichte Typus ist lokal beschränkt nur an Bögen der Wangenreliefs in Jacobi zu finden. Stark verschattet, fallen die Teilblätter dort kaum ins Auge - dies erklärt auch, warum sie im Vergleich mit den Dreiblatttypen an den Dorsalen in Nikolai gröber ausgearbeitet sind. Trotzdem sind ihnen systemtypische Formen eigen, durch die sie sich in die Transformationsreihe einfügen. Zugleich sind sie ein Beleg für die frühen Rationalisierungsmöglichkeiten und intendierten Formvarianzen innerhalb einer Reihe.

\section{Genealogische Reihe}

Jedes der untersuchten Chorgestühle markiert mit seinen Kriechblumen eine Reihe, die durch die Betrachtung von außen, also vom heutigen Standpunkt aus, geschlossen und endlich ist. In das objektübergreifende Ornamentsystem eingebunden, verringert sich laut Kubler mit jeder ausgeführten Kriechblume die Anzahl sowie die mögliche Position der nachfolgenden Kriechblumen. ${ }^{9}$ Somit legt die zuerst ausgeführte Kriechblume am Wimpergschenkel die Form der folgenden am Bauteil und gegebenenfalls auch im gesamten Gestühlsgefüge fest und ist Ausgangspunkt für eine genealogische Reihe. Wenn wir Genealogie als repetitive Materialisierung von Informationen verstehen, so steckt in jeder Kriechblume sowohl die Information über ihre Form und deren Abhängigkeit von anderen Formen wie auch über ihre Entstehung durch die handwerkliche Technik. Eine Genealogie kann in Nikolai für die einzelnen Wimpergmotive festgelegt werden: Seitlich angeschnittene und mit dem Rahmenprofil kollidierende Kriechblumen belegen, dass bei jedem Dorsalfeld die bekrönende Blume zuerst und dann den Wimperg abwärts gearbeitet wurde. ${ }^{10}$

9 Siehe Kublers unter Anm. * zitierte Definition einer Reihe. Mit mindestens sechs Kriechblumen und zwei Kreuzblumen besetzt, weist jeder Kielbogen die gleiche Struktur auf.

10 Siehe exemplarisch die Kriechblumen der Südreihe (Nikolai_R1_D1 bis R1_D16 sowie R1_a_W1; R1_i_W1 in Abb.4; $\mathrm{R}=$ Reihe, $\mathrm{D}=$ Dorsale, $\mathrm{W}=$ Wange, $\mathrm{a}=$ außen, $\mathrm{i}=$ innen $)$. 
Bei artifiziellen Gegenständen wie dem hier untersuchten Chorgestühl beruhen Ähnlichkeiten im Ornamentsystem nicht auf Zufällen. Vielmehr sind die bewusste Wiederkehr und Abwandlung des Formenspektrums vorauszusetzen; Nikolai mit seinem regelmäßigem System einer alternierenden Reihe belegt diese Annahme.

Da mittelalterliche Kunstobjekte und deren wiederkehrende Ornamente Einzelanfertigungen sind, ist es kaum verwunderlich, dass auch auf den ersten Blick gleichförmige, an einem Wimperg angebrachte Kriechblumen desselben Typs in den Merkmalszuständen stark divergieren. In dieser Differenz lässt sich ein schleichender Formwandel greifen, den Kubler als »tiny unwanted variations «" beschreibt, die bei der wiederholten Ausführung einer Replik - und nichts anderes ist das Schnitzen einer Kriechblume nach dem Modell der ersten Kriechblume am Bogen - unvermeidbar und zufällig auftreten. Denn jeder wiederholte Schnitzakt unterscheidet sich leicht vom vorhergehenden, ${ }^{12}$ sei es durch unregelmäßige Strukturen des bearbeiteten Materials oder durch den Einfluss des ausführenden Schnitzers und dessen persönlichen Befindens bzw. seines latenten Wunsches nach Abwechslung. Dies betrifft vor allem einzelne Kriechblumen in Jacobi, die den gleichen Merkmalszustand wie entsprechende Objekte in Nikolai zeigen, aber deutlich mehr Abweichungen innerhalb einer Sitzreihe aufweisen. Besonders auffällig ist das unvermittelte Auftreten dreier verschiedener Kriechblumentypen am Bogen des Wangenreliefs Jacobi_R3_W2. Das bewusste Kopieren bei gleichzeitiger Abwandlung spiegelt vermutlich die Absicht des Schnitzers, die auf die Kirche bezogene genealogische Reihe zu durchbrechen.

\section{Methodologische Probleme}

Während sich kleine Unterschiede möglicherweise aus dem Zufall ergeben können, müssen wir größere, besonders aber systematische Unterschiede wie jene alternierende Verwendung zweier Typen in Nikolai der Intention zurechnen. Sie spiegeln das bewusste Spiel mit dem Formwechsel innerhalb einer Reihe. Die geringe Differenz der Merkmalszustände belegt darüber hinaus die beabsichtigte homogene Ausführung. Das reiche Formenspektrum in Jacobi hingegen mag zumindest bei der Wahl der Kriechblumenformen an den Wangen intendiert sein. Die heterogene Verteilung der Merkmalszustände wie auch der Kriechblumentypen an den Dorsalen sprechen indes für einen wahllosen oder zumindest weniger intentionalen Rückgriff auf die bereits in Nikolai angelegten Formen. Beides, Intention und Zufall, lässt sich mit dem phylogenetischen Werkzeug wie auch in der konventionellen Kunstgeschichte nach Stilen bislang nicht ausreichend beschreiben und adäquat abbilden.

11 Kubler 1963, 71.

12 Kubler 1963, 73: »Each act varies slightly from the preceding.« 
Die Untersuchung ist noch nicht abgeschlossen, denn es wurden bisher nur die Kreuzblumen vergleichend untersucht. Dadurch aber ist der Wesenszug der Kriechblumen von St. Nikolai als alternierende Reihe nur zum Teil erfasst. Gerade die Dorsalfelder mit den blattartigen Kreuzblumen auf den Wimpergen zeigen eine interne Varianz, die auf eine Verkomplizierung der Formensprache hinweist. Die an Schenkeln derselben Wimperge sitzenden Kriechblumen vom Dreiblatttyp, deren einzelne Teilblätter in alle Richtungen greifen, wirken dadurch, als seien sie weitestgehend richtungslos gewachsen. Bei den bekrönenden Kreuzblumen hingegen wird durch das Umlegen des Rückenblattes nach oben nicht nur der Dreiblatt- in einen Zweiblatttyp gewandelt, sondern auch eine absolute Richtung festgelegt (Abb. 7 rechts). Die Blätter greifen ausschließlich nach oben und erzeugen somit eine das Architekturmotiv abschließende Bekrönung. Eine derartige Differenzierung und zugleich Hierarchisierung der verwendeten Typen ist an den Dorsalen mit durchgängiger Verwendung des schneckenförmigen Zweiblatttypus nicht erfolgt (Abb. 7 links), kann aber vereinzelt auch an Dorsalen und Wangen des Jacobigestühls ausgemacht werden. ${ }^{13}$

\section{Fazit}

Grundsätzlich lässt sich auf Basis der bisherigen Auswertung festhalten, dass sich die aus den Kladogrammen ablesbaren Ergebnisse überraschend gut mit den Resultaten kunsthistorischer Beobachtungen decken.

Gegenüber dieser Arbeitsweise, bei der oftmals nur einige wenige, als wesentlich erachtete Merkmale für den Vergleich herangezogen werden, bietet die phylogenetische Methode den Vorteil der reproduzierbaren Begutachtung aller systemtypischen Eigenschaften. Die diagrammatische Darstellung der Abhängigkeiten in Form von Kladogrammen kann die Kunsthistoriker_innen bei der Auswertung von Untersuchungen und der Entwicklung von Fragestellungen unterstützen. Angewendet auf eine klar abgegrenzte Werkgruppe, erstellt die phylogenetische Methode Serien und Transformationsreihen, mittels derer interne Genealogien und somit die anhand von Stilveränderungen vorgeschlagene Datierung abgesichert werden können. Zugleich kann sie neue Impulse für die Interpretation von Artefakten liefern. ${ }^{14}$

Während die vorgenannten Objekte einer Werkstatt zugerechnet werden, ist in einem nächsten Schritt zu prüfen, ob die Methode auch bei Werken verschiedener Urheberschaft unter Berücksichtigung größerer Zeiträume und Formenphänomene tragfähig ist.

13 Vgl. Jacobi_R2_W2, W3, D2 und D6 sowie R3_W1 und D6.

$14 \mathrm{Vgl}$. Scholtz/Scholtz 2013, 5-8. 


\section{Literatur}

Arens, Fritz Viktor (1948): Bosse, Bossenkapitell. In: Schmitt, Otto (Hg.): Reallexikon zur deutschen Kunstgeschichte, Bd.II. Stuttgart: Alfred Druckenmüller, Sp. $1062-1066$.

Bucher, François (1979): Architector. The Lodge Books and Sketchbooks of Medieval Architects, vol. 1. New York: Abaris Books.

Geldner, Ferdinand (Hg.) (1965): Matthäus Roriczer: puechlen der fialen gerechtikait, Regensburg 1486, und Die Geometria Deutsch, um 1487/88. Faksimileausg. und Textübertr. Wiesbaden: Pressler.

Goloboff, Pablo/Farris, Steve/Nixon, Kevin (2000): TNT (Tree Analysis Using New Technology)(BETA) ver. xxx Published by the authors, Tucumán, Argentina. Online: http://www.cladistics.com (last access: 26 February 2015)

Kubler, George (1963): Shape of Time. Remarks on the History of Things. 2. ed. New Haven/London: Yale University Press.

Lemper, Ernst-Heinz (1950): Das Astwerk. Seine Formen, sein Wesen und seine Entwicklung. Diss., Universität Leipzig.

Maddison, Wayne/Maddison, David (2014): Mesquite: A Modular System for Evolutionary Analysis. Version 3.01. Online: mesquiteproject.org (last access: 26 February 2015).
Rathe, Kurt (1926): Ein Architekturmusterbuch der Spätgotik mit graphischen Einklebungen. In: Festschrift der Nationalbibliothek Wien. Wien: Nationalbibliothek Wien, S. $667-692$

Riegl, Alois (1893): Stilfragen, Grundlegungen zu einer Geschichte der Ornamentik. Berlin: von Georg Siemens. Roth, Hermann Josef (1976): Die Pflanzen in der Bauplas tik des Altenberger Domes. Ein Beitrag zur mittelalterlichen Botanik. Bergisch Gladbach: Heider.

Sachs, Hannelore (1960): Die mittelalterlichen Chorgestühle in der Altmark und in Havelberg. Diss., Hum boldt-Universität zu Berlin.

Scholtz, Gerhard (2013): Versuch einer analytischen Morphologie. In: Bredekamp, Horst/Bruhn, Matthias/ Werner, Gabriele (Hg.): Bildwelten des Wissens. Kunsthistorisches Jahrbuch für Bildkritik, Bd. 9.2: Morphologien. Berlin: Akademie Verlag, S.30-44.

Scholtz, Gerhard/Scholtz, Fabian (2013): Phylogenetische Analyse von Kunst. Bericht über den LunchTalk am 24. Oktober 2013 In: Cluster-Zeitung CZ, \#45, S. 5-8.

Strohmayer, Wolfgang (2004): Matthäus Roriczer-Baukunst Lehrbuch. Hürtgenwald: Pressler.

Wissell, Rudolf (1986): Des alten Handwerks Recht und Gewohnheit (Einzelveröffentlichungen der Historischen Kommission zu Berlin, 7). Hg. v. Schraepler, Ernst. 2. Aufl. Berlin: Colloquium Verlag. 


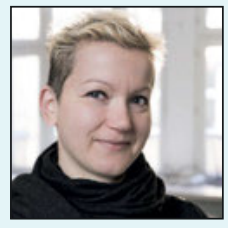

seligera@cms.hu-berlin.de

Wissenschaftliche Mitarbeiterin

Basisprojekt: Genese \& Genealogie von Form

Disziplin: Kunst- und Bildgeschichte

Anja Seliger studierte nach Lehr- und Arbeitsjahren im Tischlerhandwerk Kunstgeschichte sowie Ur- und Frühgeschichtliche Archäologie an der Humboldt-Universität zu Berlin. In ihrer laufenden Dissertation untersucht sie die Gestaltung mittelalterlicher Chorgestühle in der Mark Brandenburg im Kontext liturgischer Anforderungen und der Disposition im Sakralraum.

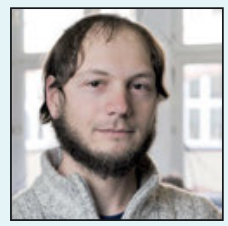

guenther.jirikowski@hu-berlin.de

Wissenschaftlicher Mitarbeiter

Basisprojekt: Genese \& Genealogie von Form

Disziplin: Biologie

Günther Jirikowski hat an der Universität Jena Biologie studiert und an der Universität Rostock zur Evolution der Mesoderm- und Muskelentwicklung der höheren Krebse (Malacostraca) promoviert. Als wissenschaftlicher Mitarbeiter am Exzellenzcluster Bild Wissen Gestaltung forscht er über die Entwicklung und Evolution der Krebstiere sowie die Anwendung biologischer Konzepte von Formbildung und -wandel in der Kunstgeschichte und den Gestaltungswissenschaften.

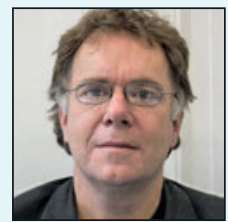

gerhard.scholtz@rz.hu-berlin.de

Principal Investigator

Basisprojekte: Attention \& Form, Genese \& Genealogie von Form, Historische Strukturen, Selbstbewegende Materialien

Disziplin: Biologie

Gerhard Scholtz ist Professor für Vergleichende Zoologie und Leiter der Zoologischen Lehrsammlung an der Humboldt-Universität zu Berlin. Er ist Mitglied des Exzellenzclusters Bild Wissen Gestaltung. Ontogenese und Phylogenese der Gliederfüßer sowie theoretische Fragen zur Evolution der Form bilden die zentralen Themen seiner Forschung. 



\section{Wahrnehmung von Datengrafiken}

\section{Ein verzerrter Eindruck?}

Datengrafiken sind wissenschaftliches Alltagsgeschäft. Bei der Vermittlung von wissenschaftlichen Ergebnissen an die Öffentlichkeit und Prozessen der politischen Meinungsbildung spielen sie eine zunehmend große Rolle. Dass Datengrafiken verständlich sind und die Aussagen gut transportieren, ist daher ein zentrales Ziel. Ihre Gestaltung hat an Bedeutung gewonnen, denn sie beeinflusst die Erfassung der dargestellten Ergebnisse.' Selbst teils arbiträre perzeptuelle Eigenschaften bestimmen Aufmerksamkeit, Verständnis und Gedächtnis für Grafik und Inhalt mit. Daher bietet die psychologische Forschung zu den Stärken und Schwächen der visuellen Wahrnehmung hilfreiche Informationen zur Gestaltung. Der vorliegende Beitrag argumentiert, dass sich die Eigenschaften menschlicher (visueller) Wahrnehmung auf Erfolge, Wege und Umwege des wissenschaftlichen Arbeitens auswirken. Wir rufen dazu auf, Datengrafiken zum zentralen Gegenstand wahrnehmungspsychologischer Arbeiten in der empirischen Wissenschaftsforschung zu machen. Wir gehen aufdrei Aspekte ein: 1) Die Bedeutung und Entwicklung von Datengrafiken in der Wissenschaft, 2) die Relevanz von Psychologie für ihre Gestaltung sowie 3) Beispiele für Wahrnehmungsverzerrungen, die der Konkretisierung der oben genannten Punkte dienen.

1 Vgl. Fischer/Dewulf/Hill 2005; Huestegge/Philipp 2011. 


\section{Bedeutung und Entwicklung von Datengrafiken in der Wissenschaft}

Die zweite Hälfte des 19. Jahrhunderts wird auch das Goldene Zeitalter der statistischen Datengrafiken genannt. ${ }^{2}$ Die systematischen Datensammlungen durch den Staat und die Entwicklung statistischer Theorien zur Verarbeitung von Daten bildeten in dieser Zeit einen optimalen Nährboden zur Entwicklung innovativer Formate von Datengrafiken. ${ }^{3}$ Nicht nur im gesellschaftlichen Leben ist die Bedeutung von Datengrafiken gestiegen, auch in der Wissenschaft haben sie Spuren hinterlassen. Ihr Gebrauch in wissenschaftlichen Veröffentlichungen ist während des 20. Jahrhunderts gestiegen. ${ }^{4}$ Wissenschaftler_innen kommunizieren vermehrt über Datengrafiken, sowohl in Veröffentlichungen als auch bei Konferenzen. ${ }^{5}$ Die Verbindung zwischen Wissenschaft und Datengrafiken ist heute so stark, dass selbst Menschen mit hoher Expertise wissenschaftliche Ergebnisse in Artikeln mit Datengrafiken plausibler empfinden als ohne dieselben. ${ }^{6}$ Die Disziplinen unterscheiden sich jedoch stark im Ausmaß der Nutzung von Datengrafiken. Bei einer von Laurence D. Smith und Kolleg_innen durchgeführten Untersuchung von einschlägigen wissenschaftlichen Veröffentlichungen unterschiedlicher Disziplinen konnte gezeigt werden, dass der Anteil der mit Datengrafiken bedruckten Fläche im Verhältnis zum Text über Disziplinen und Subdisziplinen hinweg stark variiert. ${ }^{7}$ Das Ergebnis zeigt, dass in den Naturwissenschaften mehr Grafiken verwendet werden als in den Geistes- und Sozialwissenschaften. Die Verwendung von Datengrafiken spiegelt sich auch in einer hohen Variabilität innerhalb der Brückendisziplin Psychologie wider, die je nach Spezialisierung eher den Geistes- bzw. Sozialoder Naturwissenschaften zugeschrieben werden kann und dementsprechend wenige oder viele Grafiken verwendet. Werden wenige Datengrafiken genutzt, muss das nicht heißen, dass keine quantitativen Daten vorhanden sind. Am Beispiel der verschiedenen Spezialisierungen innerhalb der Psychologie zeigten Smith und Kolleg_innen in einer weiteren Untersuchung, dass nur eine andere Form der Darstellung gewählt wurde. ${ }^{8}$ Wurden weniger Datengrafiken verwendet, dann kamen entsprechend mehr Tabellen vor, in denen man Werte konkret ablesen kann. Statistische Kennwerte in Tabellen bieten die Möglichkeit einer auf expliziten, mathematisch formalisierten Annahmen beruhenden Gewichtung der Abweichung zwischen Modellvorhersagen und Messwerten. Bei Datengrafiken verlassen wir uns auf unseren visuellen Eindruck vom Ausmaß der Abweichung der Daten von den quantitativen Modellvorhersagen. Die Eigenschaften der mathematisch formalisierten Abweichungsmaße sind aus der Formalisierung heraus bekannt, die Eigenschaften davon, was bei visuellem Eindruck als (starke) Abweichung wahrgenommen wird, müssen wir empirisch herausarbeiten. Durch die Präsentation von Daten in Form von Grafiken sind somit andere Aspekte von Daten ausschlaggebend dafür, ob wir eine Annahme

2 Siehe Friendly 2008.

3 Siehe ebd.

4 Siehe Gross/Harmon/Reidy 2002.

5 Siehe Cleveland 1984.

6 Siehe Isberner et al. 2013.

7 Siehe Arsenault/Smith/Beauchamp 2006; Kubina/Kostewicz/Datchuk 2010; Smith et al. 2000

8 Siehe Smith et al. 2002. 
für bestätigt oder widerlegt halten. Darum ist es wichtig zu erforschen, wovon dieser Eindruck abhängt bzw. wie sich die Gewichtung von Abweichungen a) in Verfahren der Inferenzstatistik und b) beim Betrachten von Daten in Streudiagrammen etc. unterscheiden.

\section{Wahrnehmungspsychologische Grundlagen der Gestaltung von Datengrafiken}

Der Psychologe William F. Brewer illustriert die Eigenschaften der menschlichen visuellen Wahrnehmung anhand von Beispielen der Wissenschaftsgeschichte ${ }^{9}$. Diese zeigen, dass Erwartungen bzw. Erfahrungen von Forscher_innen stark ins Gewicht fallen. Beispielsweise fällten Astronom_innen auch nach der Einführung des Fernrohres ihre Urteile auf Basis schwacher visueller Eindrücke an der Grenze des visuell Wahrnehmbaren; ein vermeintlicher Komet wurde im Beobachtungsprotokoll über die Nächte größer; ein Mond wurde nicht als solcher wahrgenommen, weil er unerwartet dicht den Planeten umkreiste. Schwache bzw. verschwommene Wahrnehmungsinhalte in der Astronomie haben die Entwicklung der Methodik und Fragestellungen der Psychophysik maßgeblich vorangetrieben - eines Feldes, in dem sich Forscher_innen mit Abhängigkeit zwischen der physikalischen Intensität und der Stärke der Empfindung eines Reizes bzw. seiner Detektierbarkeit beschäftigen.

Die durch Datengrafiken vermittelte Beobachtung nimmt gegenüber direkter Beobachtung eine zunehmend große Rolle ein. Von Erwartungen und Erfahrungen bestimmte Urteile am Rande der Wahrnehmungsschwelle sollten also nicht davon ablenken, dass das Tagesgeschäft heutiger Wissenschaftler_innen aus vermittelter Beobachtung besteht. Daher bedarf es einer genaueren Erforschung der Wahrnehmungsprozesse, die bei der Beobachtung mittels Datengrafiken relevant sind. Einerseits wirken hier die in der Gestaltpsychologie beschriebenen universellen Gruppierungsprinzipien. Beispielsweise werden Objekte als Gruppe bzw. zusammengehörig wahrgenommen, die nah beieinanderliegen oder einander ähnlich sind. Balken, die in Balkendiagrammen nebeneinanderstehen, können selbst dann als zusammengehörig wahrgenommen werden, wenn die Sortierung der Kategorien bzw. Balken weitgehend arbiträr ist. Zudem wirken Erfahrung bzw. Erwartung auf die Wahrnehmung ein. Die Wissenschaftspsychologie sollte also das Augenmerk darauf legen, wie Datengrafiken wahrgenommen werden. Gestaltung und Auswahl derselben zählen zur alltäglichen Praxis und Herausforderung vieler Wissenschaftler_innen.

Es existieren umfangreiche Untersuchungen zu einzelnen Eigenschaften unterschiedlicher Arten von Datengrafiken. Zum Beispiel zeigen Balken-Vorteile gegenüber Tortendiagrammen, da die Ergebnisse aus ihnen schneller und korrekter herausgelesen werden können. Einfache Balkendiagramme sind besser als geteilte ${ }^{10}$ und vertikale sind benutzerfreundlicher als horizontale."

9 Siehe Brewer 2012.

10 Siehe Simkin/Hastie 1987.

11 Siehe Fischer/Dewulf/Hill 2005. 
Während Balkendiagramme besser für diskrete Werte geeignet sind, kann man Trends sehr gut an Liniendiagrammen ablesen - dies bestätigen auch die Ergebnisse von Zacks und Tversky. ${ }^{12}$ Sie fragen ferner, inwieweit es sich dabei um etwas kognitiv Natürliches handelt oder um das Ergebnis kommunikativer Konventionen.

\section{Erste eigene Ergebnisse aus Experimenten zur Wahrnehmung von Datengrafiken}

Balkendiagramme können leider nicht als best practice für alle Aussagen verwendet werden, zeigte sich doch eine Unterschätzung der Werte in dieser Darstellungsform. ${ }^{13}$ David Peebles verglich Linien-, Balken- und Netzdiagramme und ließ seine Versuchspersonen beurteilen, wie viel besser oder schlechter der Wert einer Dimension im Vergleich zum Mittelwert war. In Balkendiagrammen wurden die Werte systematisch unterschätzt. Eine Ursache könnte darin liegen, dass Menschen die Länge von vertikalen Balken überschätzen. ${ }^{14}$

Ziel unserer Studie ist zu prüfen, ob Balkendiagramme einen verzerrten Eindruck der zentralen Tendenz (Mittelwert) vermitteln. Hierzu entwickelten wir im Besonderen ein Experiment, bei dem wir die Länge der Balken variierten und die Wahrnehmung von Balken und Punkten verglichen. 53 Versuchspersonen nahmen an diesem Experiment teil. Ihnen wurden in vier Blöcken jeweils 80 Datengrafiken nacheinander sehr kurz präsentiert. Zum Beispiel wurden ihnen Balkendiagramme mit acht grauen Balken und einer rot eingezeichneten Linie vorgelegt, die den Mittelwert angibt (Abb. 1). Die Aufgabe bestand darin, den Mittelwert zu bestimmen und so schnell wie möglich zu entscheiden, ob die rote Linie eigentlich höher oder tiefer liegen müsse. Die gegebene Antwort und die Reaktionszeit wurden erfasst. In den vier Versuchsabschnitten wurden verschiedene Merkmale variiert. Zum Beispiel wurde die Wirkung hoher und niedriger Balken verglichen.

Die Ergebnisse zeigten, dass bei Balkendiagrammen im Vergleich zu Punktdiagrammen der Mittelwert systematisch tiefer gesehen wird, als er eigentlich ist. ${ }^{15}$ Das heißt, Balkendiagramme können einen verzerrten Eindruck der zentralen Tendenz vermitteln. Die Länge der Balken hatte keinen Einfluss auf die Unterschätzung und kann somit diese Verzerrung nicht erklären. Nach der Unterschätzung von Einzelwerten im Vergleich zum Mittelwert ${ }^{16}$ konnten wir nun eine Unterschätzung des Mittelwertes in Balkendiagrammen nachweisen. Trotz dieser Wahrnehmungsverzerrung, die sich speziell bei Balkendiagrammen zeigt, sollten diese weiter genutzt werden. Denn in anderen Settings, zum Beispiel um Beziehungen zwischen drei Variablen zu

\footnotetext{
12 Siehe Zacks/Tversky 1999.

13 Siehe Peebles 2008.

14 Siehe Jarvenpaa/Dickson 1988; Kosslyn 2006.

15 Vgl. Gaschler/Vogelgesang/Godau 2014.

$16 \mathrm{Vgl}$. Peebles 2008.
} 


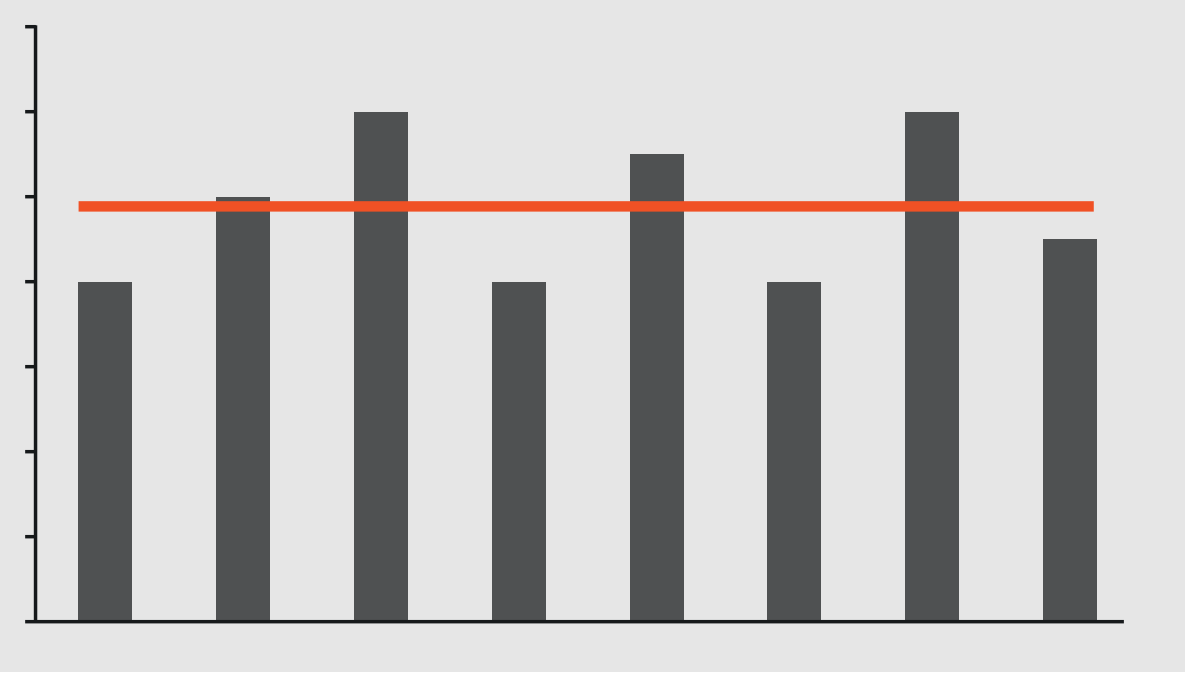

Abb. 1: Experiment zur Wahrnehmung von Balkendiagrammen, Beispiel aus dem Aufgabenmaterialset.

zeigen, sind Balkendiagramme zu bevorzugen, da es im Vergleich zu Liniendiagrammen zu weniger Fehlern bei der Interpretation kommt. ${ }^{17}$

\section{Fazit}

Zusammenfassend zeigt sich, dass 1) Datengrafiken eine Form der Visualisierungen von wissenschaftlichen Ergebnissen sind, die stetig zugenommen hat. Datengrafiken helfen, komplexe Zusammenhänge schneller und besser zu kommunizieren; 2) die Psychologie sich mit dem Thema Wahrnehmung von Datengrafiken beschäftigen sollte, da es 3) zu Wahrnehmungsverzerrungen beim Lesen von Datengrafiken kommen kann, wie am Beispiel von der Unterschätzung des Mittelwertes gezeigt werden konnte.

Untersuchungen zur Gestaltung von Datengrafiken sollten - so auch William Cleveland und Robert McGill - interdisziplinär umgesetzt werden. ${ }^{18}$ Durch die Kombination von Geistes-, Sozial-, Naturwissenschaften und Gestaltungsdisziplinen bietet der Exzellenzcluster Bild Wissen Gestaltung hierfür die optimale Grundlage. Die Ergebnisse unserer Experimente können in die Gestaltung von Datengrafiken einfließen. Datengrafiken ersetzen zunehmend Tabellen mit statistischen Kennwerten. Über deren statistische Grundlagen gibt es elaborierte Abhandlungen in Fachveröffentlichungen und Lehrbüchern. Wir müssen unseren Wissensstand zu Datengrafiken also dringend auf ein ähnliches Niveau bringen, damit unsere Schlüsse wieder auf bekannten Grundlagen basieren. 


\section{Literatur}

Arsenault, Darin J./Smith, Laurence D./Beauchamp Edith A. (2006): Visual Inscriptions in the Scientific Hierarchy Mapping the »Treasures of Science«. In: Science Communication, vol.27, no. 3 , pp. $376-428$. Online: http://scx.sagepub.com/content/27/3/376.full.pdf+html (last access: 30 March 2015).

Brewer, William F. (2012): The Theory Ladenness of the Mental Processes Used in the Scientific Enterprise. In: Proctor, Robert W./ Capaldi, E. John (eds.): Psychology of Science: Implicit and Explicit Processes. New York: Oxford University Press, pp. 290-233.

Cleveland, William S. (1984): Graphs in Scientific Publications. In: The American Statistician, vol. 38, no.4, pp. $261-$ 269. Online: http://amstat.tandfonline.com/doi/pdf/10.10 80/00031305.1984.10483223\# (last access: 30 March 2015).

Cleveland, William S. / McGill, Robert (1987): Graphical Perception: The Visual Decoding of Quantitative Information on Graphical Displays of Data. In: Journal of the Royal Statistical Society. Series A (General), vol. 150, no. 3, pp.192-229. Online: http://www.jstor.org/stable/ 2981473?seq=1\#page_scan_tab_contents (last access: 30 March 2015).

Fischer, Martin H./ Dewulf, Nele / Hill, Robin L. (2005): Designing Bar Graphs: Orientation Matters. In: Applied Cognitive Psychology, vol. 19, no. 7, pp. 953-962. Online: http://onlinelibrary.wiley.com/doi/10.1002/acp.1105/epdf (last access: 30 March 2015).

Friendly, Michael (2008): The Golden Age of Statistical Graphics. In: Statistical Science, vol. 23, no.4, pp.502-535. Online: http://arxiv.org/pdf/ogo6.3979.pdf (last access: 30 March 2015).

Gaschler, Robert/Vogelgesang, Tom/Godau, Claudia (2014): Der Durchschnitt sieht unterdurchschnittlich aus Balkendiagramme können einen verzerrten Eindruck der zentrale Tendenz vermitteln. In: Onur Güntürkün ( $\mathrm{Hg}$.): 49. Kongress der Deutschen Gesellschaft für Psychologie. Abstracts. Bochum: Pabst Science Publisher, S.64.

Gross, Alan G./Harmon, Joseph E./ Reidy, Michael S. (2002): Communicating Science: The Scientific Article From the $17^{\text {th }}$ Century to the Present. Oxford: Oxford University Press.
Huestegge, Lynn / Philipp, Andrea M. (2011): Effects of Spatial Compatibility on Integration Processes in Graph Comprehension. In: Attention, Perception, \& Psychophysics, vol.73, no. 6, pp.1903-1915. Online: http://link. springer.com/article/10.3758/s13414-011-0155-1/fulltext. html (last access: 30 March 2015).

Isberner, Maj-Britt et al. (2013): Comprehending Conflicting Science-Related Texts: Graphs as Plausibility Cues. In: Instructional Science, vol.41, no. 5, pp. 849-872. Online: http://link.springer.com/article/10.1007/s11251-012-9261-2 (last access: 30 March 2015).

Jarvenpaa, Sirkka L. / Dickson, Gary W. (1988): Graphics and Managerial Decision Making: Research-Based Guidelines. In: Communications of the ACM, vol.31, no. 6 , pp. 764-774. Online: http://dl.acm.org/citation.cfm?id =62971 (last access: 30 March 2015).

Kosslyn, Stephen M. (2006): Graph Design for Eye and Mind. New York: Oxford University Press.

Kubina, Richard M. / Kostewicz, Douglas E./ Datchuk, Shawn M. (2010): Graph and Table Use in Special Education: A Review and Analysis of the Communication of Data. In: Evaluation \& Research in Education, vol. 23, no. 2, pp. 105-119. Online: http://www.tandfonline.com/doi/ full/10.1080/09500791003734688\#abstract (last access: 30 March 2015).

Peebles, David (2008): The Effect of Emergent Features on Judgments of Quantity in Configural and Separable Displays. In: Journal of Experimental Psychology: Applied, vol. 14, no. 2, pp. 85-100. Online: http://psycnet.apa.org/ journals/xap/14/2/85/ (last access: 30 March 2015).

Peebles, David (2011): The Effect of Graphical Format and Instruction on the Interpretation of Three-Variable Bar and Line Graphs. In: Carlson, L. / Hoelscher, C. / Shipley, T. F. (eds.): Proceedings of the $33^{\text {rd }}$ Annual Conference of the Cognitive Science Society. Austin, TX: Cognitive Science Society, pp.3143-3154. Online: http://cognitivesciencesociety.org/conference2011/index.html (last access: 2 March 2015).

Peebles, David / Ali, Nadia (2009): Differences in Comprehensibility Between Three-Variable Bar and Line Graphs. In: Taatgen, Niels A./ Rijn, Hedderik van (eds.): Proceedings of the $31^{\text {st }}$ Annual Conference of the Cognitive Science Society. Amsterdam: Cognitive Science Society, pp. 2938-2943. Online: http://csjarchive.cogsci.rpi.edu/ Proceedings/2009/index.html (last access: 2 March 2015). 
Simkin, David / Hastie, Reid (1987): An Information-Processing Analysis of Graph Perception. In: Journal of the American Statistical Association, vol. 82, no. 398, pp.454465. Online: http://www.jstor.org/stable/2289447?seq= 2\#page_scan_tab_contents (last access: 30 March 2015).

Smith, Laurence D. et al. (2000): Scientific Graphs and the Hierarchy of the Sciences: A Latourian Survey of Inscription Practices. In: Social Studies of Science, no. 30, pp. 73-94. Online: http://www.jstor.org/stablepdf/ 285770.pdf?acceptTC=true (last access: 30 March 2015).
Smith, Laurence D. et al. (2002): Constructing Knowledge. The Role of Graphs and Tables in Hard and Soft Psychology. In: American Psychologist, no. 57, pp. 749-761.

Zacks, J./Tversky, B. (1999): Bars and Lines: A Study of Graphic Communication. In: Memory \& Cognition, vol. 27, no. 6, pp. 1073-1079. Online: http://aaaipress.org/Papers/ Symposia/Fall/1997/FS-97-03/FS97-03-018.pdf (last access: 30 March 2015).

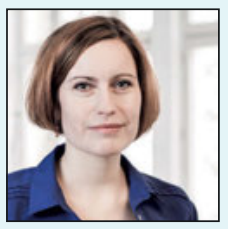

claudia.godau@hu-berlin.de

Wissenschaftliche Mitarbeiterin

Basisprojekt: Experiment \& Beobachtung

Disziplin: Psychologie

Claudia Godau hat ihren Abschluss als Diplom-Psychologin an der Universität Leipzig gemacht. Seit Mai 2012 ist sie wissenschaftliche Mitarbeiterin an der Humboldt-Universität zu Berlin. Ihre Promotion untersucht die Entwicklung mathematischer Konzepte bei Grundschulkindern. Im Cluster Bild Wissen Gestaltung erforscht sie, wie sich Wissensstrukturen zu wissenschaftstheoretischen Sachverhalten durch den Kontakt mit verschiedenen Wissenschaftsdisziplinen im Rahmen der interdisziplinären Zusammenarbeit über die Zeit verändern. Ihr zweiter Forschungsschwerpunkt liegt auf der Wahrnehmung von Datengrafiken.

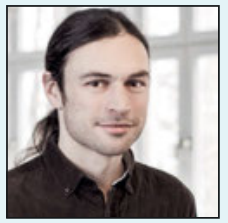

gaschler@uni-landau.de

\section{Associated Member}

Basisprojekte: Attention \& Form, Experiment \& Beobachtung, Modelle in der Gestaltung

Disziplin: Psychologie

Robert Gaschler, seit April 2015 Professor für Allgemeine Psychologie (Lernen, Motivation, Emotion) an der FernUniversität in Hagen, widmet sich als assoziiertes Mitglied des Exzellenzclusters Bild Wissen Gestaltung empirischer Wissenschaftsforschung zur Gestaltung interdisziplinärer Forschungsverbünde und zu wahrnehmungspsychologischen Fragen. Vor seiner Zeit als Juniorprofessor der Universität Koblenz-Landau (2012-2015) hat er an der Humboldt-Universität Berlin Psychologie studiert (Promotion 2009), als PostDoc gelehrt und Forschungen zu willkürlichen und unwillkürlichen Lernprozessen betrieben. 


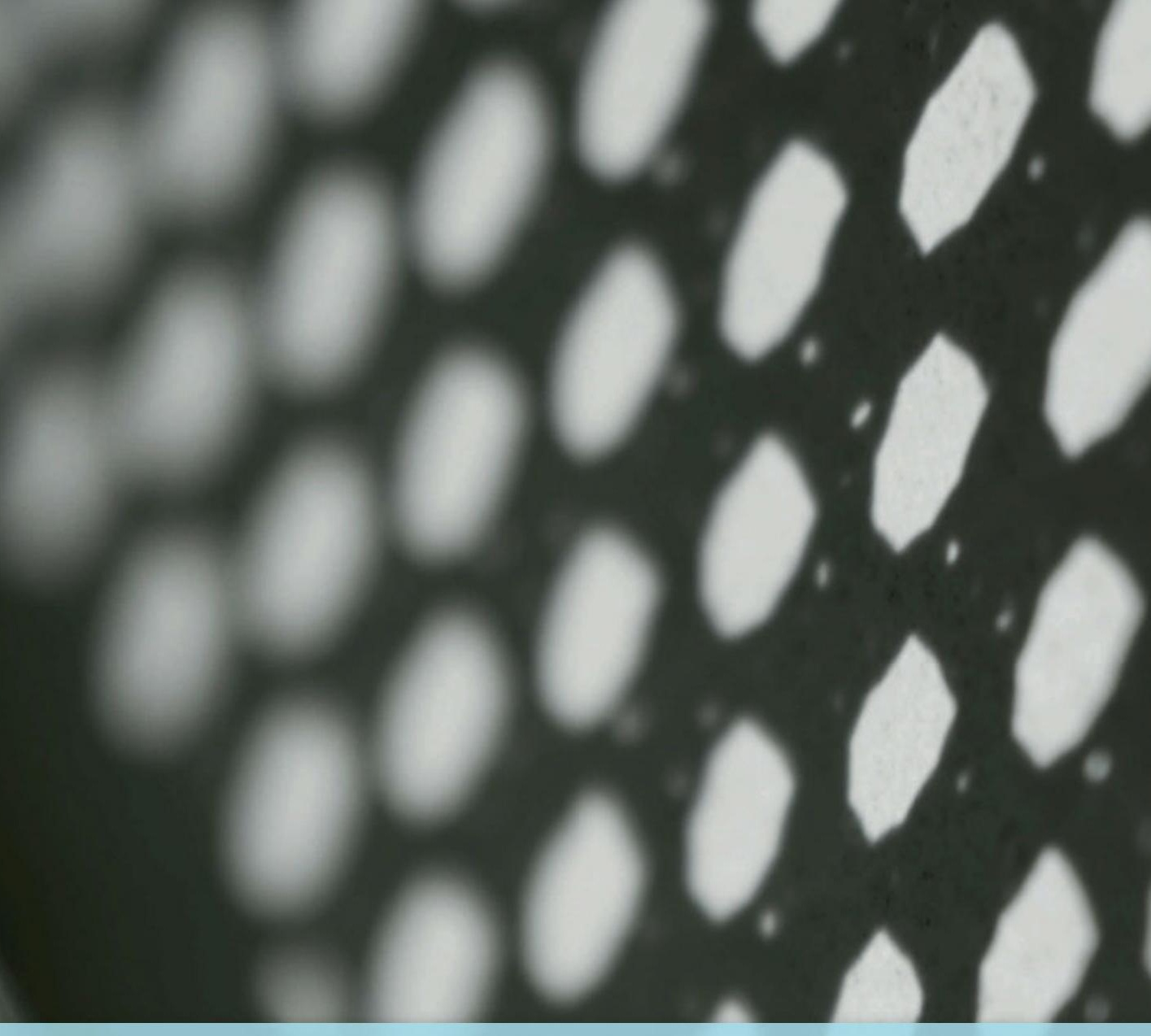

4

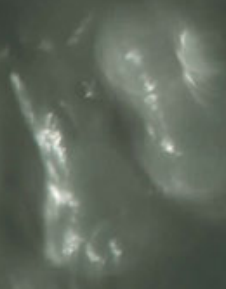

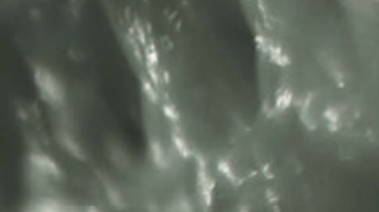
$x^{2}+x^{2} x^{2}$

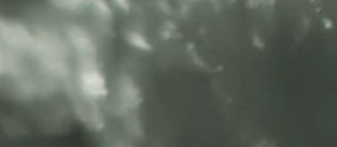
a 8

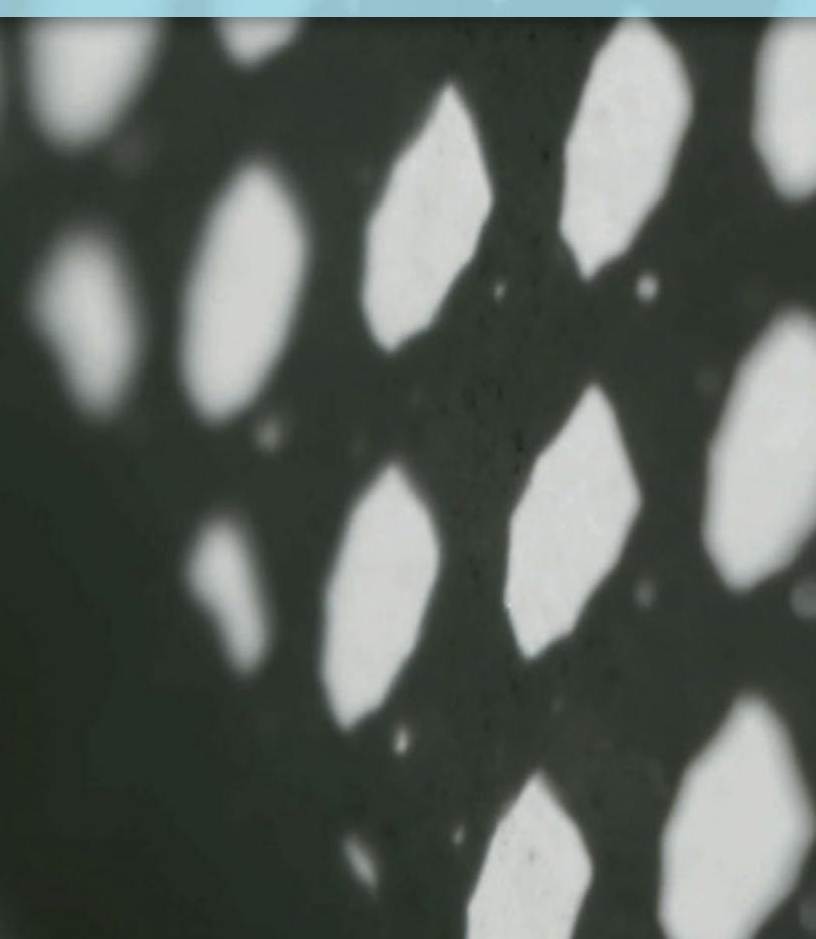




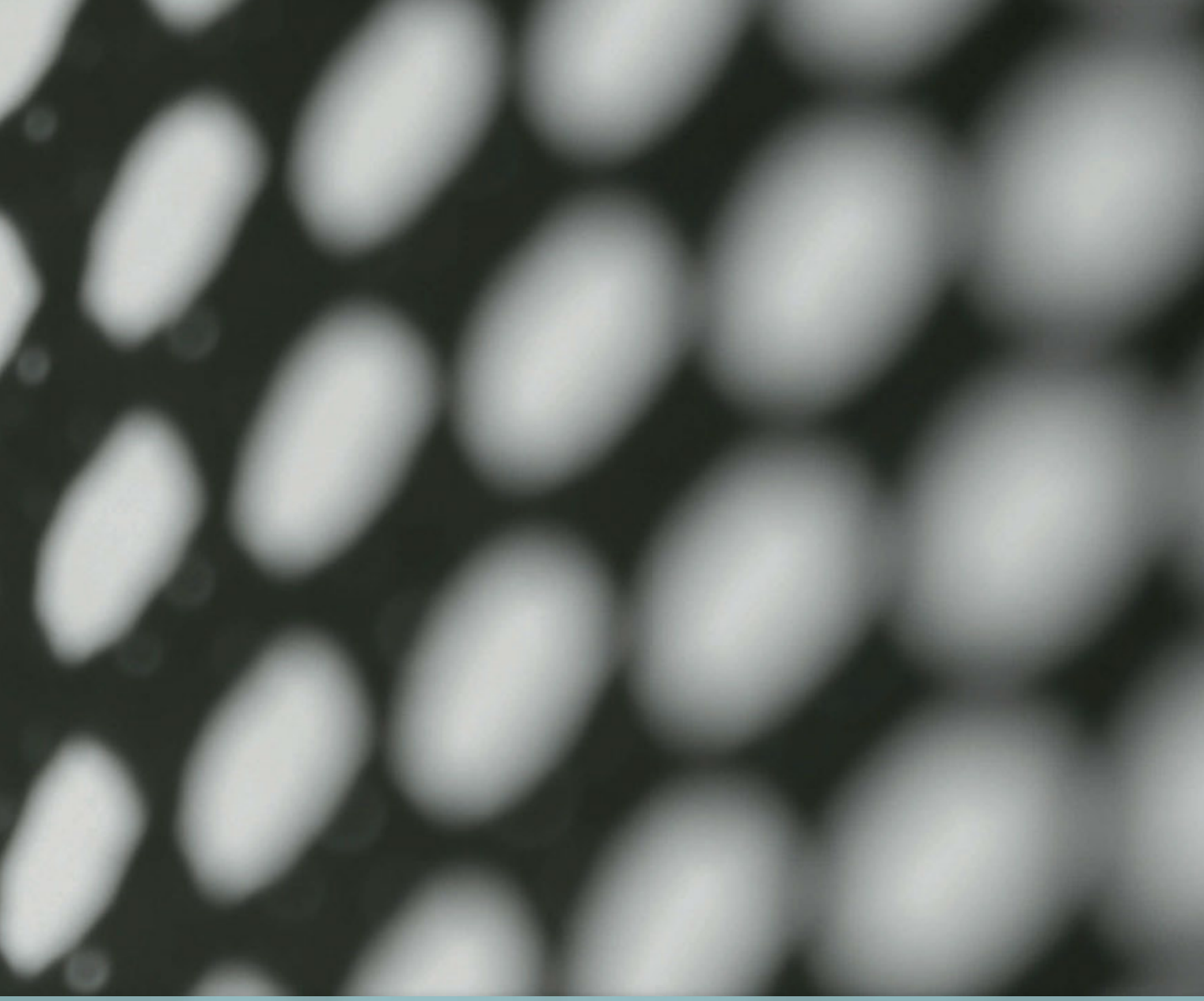

II. Wissensstrukturen

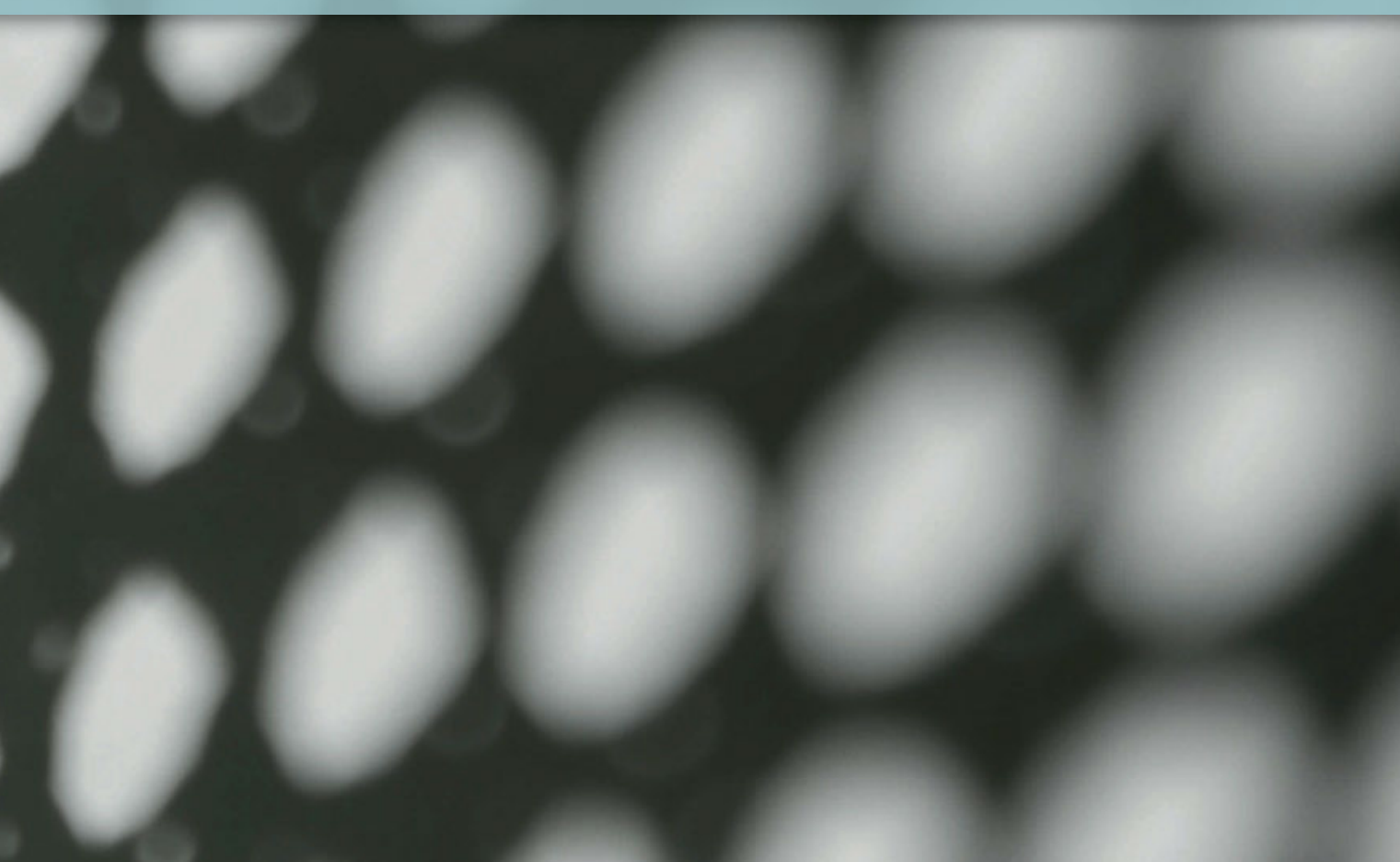




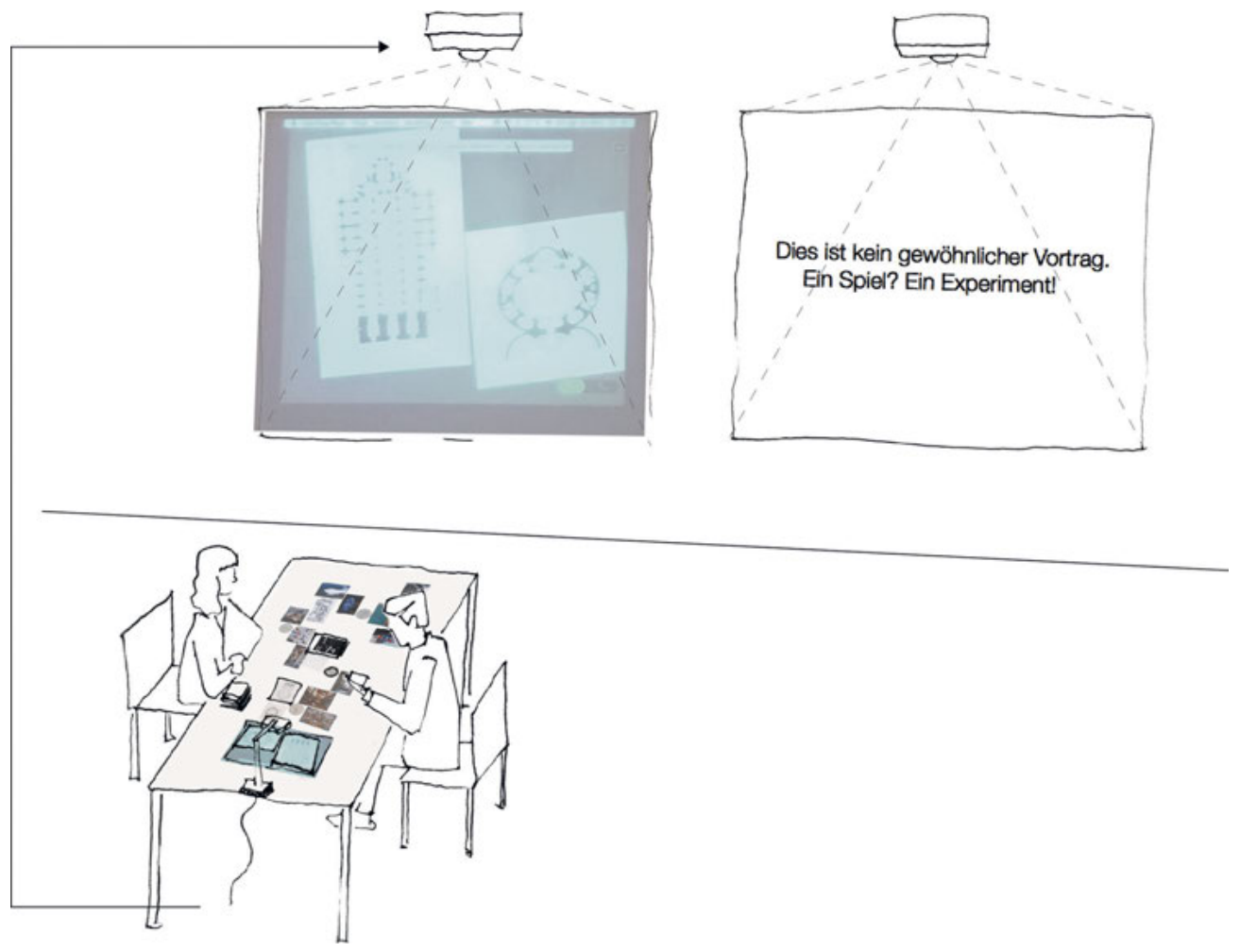

Abb. 1: Vortragssetting der Lecture Performance. 


\section{Die Entdeckung einer wissensarchitektonischen Karte}

Beim vorliegenden Text handelt es sich um die verschriftlichte Version eines Gesprächs, das Sabine Hansmann und Peter Koval im Rahmen der Cluster-Jahrestagung vor dem Publikum führten. Während dieser Lecture Performance saßen sie sich an einem Tisch gegenüber und legten abwechselnd spielkartengroße Bilder auf die Tischplatte (Abb.1). Damit auch das Publikum diese sehen konnte, wurde jedes Bild, bevor es zu den anderen gelegt wurde, kurz unter eine auf dem Tisch aufgestellte Kamera gehalten und auf eine große Leinwand projiziert. Neben dieser Leinwand lief auf einer zweiten eine automatische Präsentation. Diese zeigte im Minutentakt 20 Sätze, die einige Kontextinformationen zu der Performance lieferten. Ihrem eigenen Tempo folgend, wurden diese in die vorliegende schriftliche Version eingearbeitet.

$\mathrm{SH}$ : In den kommenden 20 Minuten möchten wir über vier Kolaboranten ${ }^{1}$ reden; über vier Akteure, welche die Beziehung von Raum und Bewegung jeweils auf ihre Art hinterfragen. Wir haben uns im Vorfeld über die Haupteigenschaften vier solcher Akteure - den Dirigenten, Mitspieler, Unterhändler und Verrückten - verständigt. Was sie aber im Konkreten bedeuten sollen, das wollen wir jetzt anhand von mitgebrachten Bildern oder auch Modellen aushandeln.

1 Wir wollen hier den Raum prosopopoetisch als einen Kolaboranten auffassen, als einen Akteur, dessen Aktionsradius sich irgendwo zwischen einem Kollaborateur auf Augenhöhe und einem lediglich dienstleistend-bereitstellenden Laboranten erstreckt. Dass wir nicht von Grenzobjekten reden, sondern Prosopopoeia bemühen, hat den Zweck, dass wir den Raum nicht nur als Ermöglicher oder Unterstützer der interdisziplinären Zusammenarbeit sehen, sondern ihm zur Kooperation und Mitautorschaft verhelfen wollen. Dirigent, Mitspieler, Unterhändler und Verrückter stellen dabei vier mögliche Kolaboranten dar. Vgl. dazu auch Joerges 1996. Obwohl > der Raum als Kolaborant< die Verwendung von Maskulin implizit einfordert, möchten wir an dieser Stelle betonen, dass wir mit jeder Erwähnung des Raums als Dirigent, als Mitspieler, Unterhändler oder Verrückter immer alle Geschlechter meinen. Grundsätzlich zielt unsere prosopopoietische Übertragung funktional auf ausgewählte Eigenschaften von allen Personen, die eine bestimmte Tätigkeit ausüben bzw. ein bestimmtes Verhalten aufweisen. 

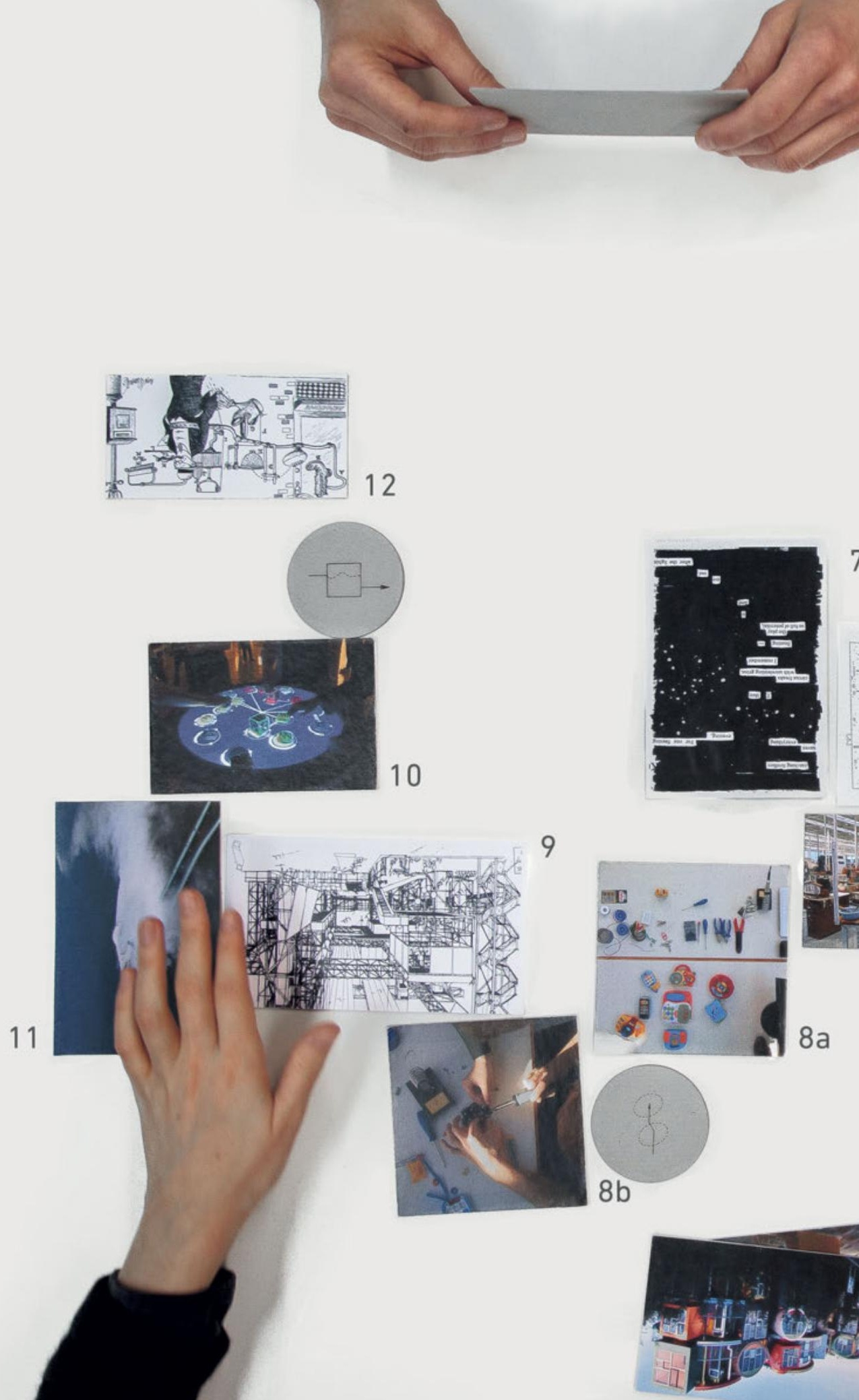
SH: Ich würde, wenn ich beginnen darf, vorschlagen, dass wir mit dem Dirigenten starten, weil er mir von all unseren Kolaboranten am klarsten ist. Ich habe zwei Dirigenten mitgebracht, und zwar zwei Kirchengrundrisse - links die St. Marienkirche in Lübeck (B2) und rechts die Sant'Andrea al Quirinale von Bernini in Rom (B 1; alle nummerierten B-Verweise beziehen sich auf die Bilder in Abb.2). Hier wird, wie ich finde, ziemlich offensichtlich, wie die Grundrisse bzw. wie die

\section{1:00 min.}

Dies ist kein gewöhnlicher Vortrag. Ein Spiel? Ein Experiment!

Gebäude die Bewegung leiten. Sie dirigieren oder choreografieren und lassen mit klaren Setzungen ganz unterschiedliche Bewegungsmuster entstehen.

Das Kirchengestühl in der St. Marienkirche leitet dabei ebenso wie gebaute Architektur die Bewegung auf den Chor zu, während bei Bernini vermutlich meist eine zirkulierend-mäandernde Bewegung entsteht. Man läuft zunächst nach vorne und dann zu den einzelnen Kapellen. Wenn diese Gebäude also Bewegungen erzeugen, dirigieren, tun sie das immer unterschiedlich, und sie haben eine ganz klare Vorstellung davon, wie sie leiten möchten.

PK: Ich musste gleich an zwei Dirigenten denken, die auf sehr unterschiedliche Art die Beziehung zwischen Raum und Bewegung in der Wissenschaft bestimmen. Wir wollen uns zwar auf den

\section{2:00 min.}

Das Ziel unseres Forschungsprojekts ist es, die wechselseitige Beziehung von Raum und Bewegung zu untersuchen.

real gebauten Raum fokussieren, dieser ist aber, wie ich finde, nicht nur von anderen >realen< Räumen in drei Dimensionen umgrenzt, sondern auch - das behaupte ich jetzt einfach so - von >symbolischen< Räumen. Und für diese Grenz- oder Übergangsräume, die genauso trennen, wie sie verbinden, sollten wir uns auch öffnen.

Eine äußerst präzise gerichtete zirkulierende Bewegung ${ }^{2}$ findet man auch in einem Teilchenbeschleuniger (B3). Das dünne Rohr, das mehrere Meter dick von Technik ummantelt ist, wäre hier sozusagen der unterste Raum der Erkenntnis. Viel passt da nicht rein, und sehen kann man da auch nicht viel. Das Interessanteste daran ist, dass es auf einen Clash, auf eine Katastrophe hin programmiert ist. Die Teilchen sollen aufeinander oder auf irgendetwas

2 Dass wir beide eine zirkulierende Bewegung in Bezug auf den Dirigenten ins Spiel brachten, war nicht Teil der vorangegangenen Absprache - es hat auch uns überrascht und in dem, was wir machen, bestärkt. Von solchen >Zufällen< war unsere Zusammenarbeit von Anfang an geprägt. 
anderes prallen und gerade durch dieses Aufeinanderprallen soll etwas entstehen. Nennen wir es Wissen.

Von der anderen Seite oder auch von >oben< sieht es ganz anders aus. Da gibt es dieses kleine Büro (B 4) mit einem

\section{3:00 min.}

Raum konstituiert Bewegung erzeugt Raum konstituiert Bewegung bestimmt Raum ...

allzu großen Tisch, auf dem sich zu viele Papiere stapeln, als dass sie von dem älteren Forscher, der auf dem Bild zu sehen ist, je bewältigt werden könnten. In diesem Raum staut sich das Wissen, welches ein ausgeklügeltes Regelwerk eigentlich in Zirkulation bringen und halten soll. Und dieses kleine Büro könnte zusammen mit dem Regelwerk den Raum der Wissenschaft von >oben< eingrenzen. Zumindest in der Europäischen Organisation für Kernforschung (CERN).

SH: War das nicht Gerd Graßhoff, der in seinem Vortrag im Frühjahr bei den Interferenzen ${ }^{3}$ die ausgeklügelten Publikationsregeln des CERN beschrieb? Ein Raum der Regeln, ein Dirigent, wie Du sagst, in dem ganze klare Anweisungen bestehen, wie man sich bewegen darf. Eigentlich handelt es sich dabei um ein Netzwerk...

PK: Genau. Dennoch gibt es da diesen Papierhaufen, oder wie soll ich es nennen, diese >Interferenz< auf dem Tisch,

\section{4:00 min.}

Aber wie?

welche die Regeln so nicht vorsehen. Woher kommt diese >Interferenz $<$ und was bringt sie uns?

Ich habe hier noch etwas, das genau zwischen diesen zwei Dirigentenpolen zu liegen scheint - zwischen dem langen >Röhrchen< und dem übervollen Tisch. Dazu eine kurze Geschichte: Das Bild zeigt das Foyer der Pixar-Studios (B5). Das Gebäude wurde damals von Steve Jobs maßgeblich mitgeplant. Jobs ursprüngliche Idee war es, die physische Bewegung der Menschen, die in dem Gebäude arbeiten, auf eine Kollision hin zu dirigieren oder auch zu orchestrieren. Mehrere 100 Mitarbeiter_innen sollten nämlich immer durch eben dieses Foyer laufen, um zu den Toiletten auf der anderen Seite zu gelangen - so wird es zumindest kolportiert.

SH: Das wurde so aber nicht gebaut, oder?

3 Sabine Hansmann bezieht sich hier auf einen Vortrag von Gerd Graßhoff, den er im Rahmen der von et al ${ }^{\circ}$ - Vereinigung Wissenschaft, Kultur und Medizin veranstalteten Ringvorlesung Interferenzen am 15. Mai 2014 im Tieranatomischen Theater Berlin hielt und bei dem es um Kollaborative Autorenschaft in den Wissenschaften ging. 


\section{5:00 min.}

An einem Pavillon als Ort der Begegnung und des Austausches für den Exzellenzcluster entwerfen wir unsere Forschungsfragen.

Idee ist für unser Projekt aber nach wie vor interessant. Denn allein durch die geschickte Raumgestaltung würden hier die Mitarbeiter_innen durch zwei asynchrone Rhythmen getaktet und so in unvorhergesehene Interaktionen aufeinander losgeschickt: Auf der einen Seite die biologischen Bedürfnisse und auf der anderen digitale Kalender, Ablaufpläne, Zeitfenster von Meetingräumen und sonstige bürokratischen Synchronisierungstechnologien.

SH: Der Dirigent wird mir immer klarer im Kontrast zu unserem weiteren Kolaboranten, dem Unterhändler. Der Unterhändler ist einer, den ich als ungerichtet verstehe - er breitet eine Art Verhandlungsraum aus und unterscheidet sich hierin vom Dirigenten. Das kann man vielleicht noch einmal an der St. Marienkirche deutlich sehen. Der Dirigent gibt Bewegungsrichtungen, -formen und -tempi klar vor (siehe B 2). Der Unterhändler hingegen bleibt offener. Ich habe ein Projekt mitgebracht, das ich

\section{6:00 min.}

Wir arbeiten interdisziplinär und ergebnisoffen.

immer wieder gerne zeige, und zwar den KAIT-Workshop (B 6a/b) von Junya Ishigami, einem jungen japanischen Architekten. Es ist ein Werkstatt- und Kommunikationsgebäude des Kanagawa Institute of Technology und besteht aus nur einem einzigen Raum. Dieser ist nahezu quadratisch und wird durch unzählige Stützen in verschiedene Zonen strukturiert. Im Grundriss sind das diese ganz kleinen Punkte. Es sieht aus wie ein Sternenhimmel oder ein Wald.

PK: Wenn also in einem Teil des Raums eine >Lichtung< ist, dann weiß der Architekt womöglich nicht, wofür sie vorgesehen ist. Ein >Dickicht< hilft hier der Verstetigung von vorher unbestimmten Prozessen ...

SH: Der Raum ist offen, aber nicht leer. Es ist etwas angedacht oder angeregt. Der Raum hat eine Struktur, aber er ist nicht vorbestimmt; er dirigiert nicht im Sinne eines Dirigenten,

07:00 $\mathrm{min}$.

Wie lassen sich Gebiete zwischen unterschiedlichen Wissenskulturen erschließen?

sondern er möchte einen Raum öffnen, er möchte gewisse Potenziale freisetzen oder entdecken. 
PK: Ich glaube, dazu habe ich hier ein Extrembeispiel.

Nehmen wir eine beliebige Zeitungsseite (B 7). Ob man es glaubt oder nicht, in jeder Spalte ist ein Gedicht verborgen. Man muss nur die richtigen Wörter schwärzen. Es ist eine ganz besondere Art der Nutzung des Buchstabenraums, die das Gedicht erscheinen lässt. Dabei ist es so einfach: Alles >Unwesentliche < wird weggelassen. Und das passiert, ohne dass die grundlegende Buchstabenstruktur, also das Darunterliegende, zerstört oder an sich verändert wird. Es kann natürlich sein, dass man den Text nachher anders betrachtet oder liest - und so ist es vermutlich auch -, nämlich als einen erweiterten Möglichkeitsraum. Die schwarze Schablone rückt den Text in die Nähe eines Unterhändlers.

SH: Aber wäre diese Form des Möglichkeitsraums dann nicht überall vorhanden?

\section{8:00 min.}

Können wir die Offenheit zu unserem Komplizen machen? (Wie lange?)

PK: Ja.

SH: ?!

PK: Das ist eine Frage der Methode, oder? Man kann vorher nicht wissen, wie der Raum nachher tatsächlich aussieht. Die Möglichkeiten sind hier aber auch nicht beliebig. Circuit Bending wäre ein gutes Modell dafür (B 8a/b): Ein Geräusche machendes Kinderspielzeug stellt in der Regel einen sehr begrenzten Raum dar. Für Kinder ist es vielleicht optimal: Fünf Knöpfe, die beim Drücken jeweils andere Geräusche machen. Außer Eltern, die so etwas enorm nerven kann, gibt es Leute, die eine Herausforderung darin sehen, die Grenzen dieses allzu kleinen Raums zu überwinden oder eher zu unterwandern. Und das funktioniert so, dass man rudimentäres Werkzeug in die Hand nimmt, die Geräte auseinanderschraubt und versucht, ihnen mit einfachen Kabeln

\section{o9:00 min.}

Wir suchten einen Raumtyp, der sich nicht durch seine Nutzung oder Form definiert, sondern wie ein aktiver Mitarbeiter und Mitautor in Erscheinung tritt.

oder auch mit den Händen, mit den Fingern auf der Platine neue Klänge zu entlocken. Und zwar durchaus unwissend, wie die Struktur des digitalen, wohlberechneten Spielzeugs eigentlich beschaffen ist. Und das funktioniert. Zerstörung bringt hier neue Strukturen hervor.

SH: Aber liegt hier nicht ein Unterschied zwischen einem Unterhändler, der einen Verhandlungsraum öffnet und ein Angebot macht, und dem Missbrauch dieses Angebots? Dann verliere ich doch den Unterhändler! 
PK: Ich weiß nicht, ob dieser Unterschied für uns so wichtig ist. Sagen wir, wir sollen jetzt einen Raum gestalten, einen Raum für Wissenschaft. Es steht uns eine Etage mit vielen kleineren Räumen zur Verfügung, und wir können im Grunde machen, was wir wollen. ${ }^{4}$ Wir können alle Wände auf der Etage abreißen und so einen einzigen großen Raum schaffen, aber auch zusätzliche Türen einbauen und so ein Labyrinth bauen. Ich will damit nur sagen, dass ein offener, unbestimmter Raum

\section{0:00 $\mathrm{min}$.}

Wir erfanden den Kolaboranten - einen Akteur, der den Raum zwischen Kollaborateur und Laborant für sich beansprucht.

tatsächlich viele Möglichkeiten verhindern kann und, umgekehrt, gerade ein Hindernis sehr produktiv sein kann. Man kann die Säulen im KAIT-Workshop, wenn man sich an ihnen stößt, nicht einfach verschieben oder absägen.

SH: Ich finde, das überschneidet sich schon mit dem nächsten Kolaboranten; und zwar mit dem Mitspieler, den ich genau als diesen >Interaktiven< verstehe: Einer, der Bewegung übersetzt, transformiert und vielleicht auch zum Tanzpartner wird. Ich habe einen Mitspieler mitgebracht, den Fun Palace (B 9), ein Projekt, das von dem Architekten Cedric Price und der Theatermacherin Joan Littlewood in den 1960er-Jahren entwickelt, aber nie realisiert wurde. Es ist kein Gebäude im konventionellen Sinne, sondern eigentlich eine interaktive Maschine. Du siehst hier dieses Stahlgerüst

\section{1:00 min.}

Folglich haben wir mehrere Kolaboranten identifiziert, welche die Beziehung von Raum und Bewegung jeweils spezifisch auffassen und als solche den weiteren Entwurfsprozess bestimmen.

mit Bodenplatten, -decken, Wandelementen und Rolltreppen etc. Es gibt auf dem Dach zwei Kräne, die ermöglichen sollen, dass diese Struktur die ganze Zeit im Wandel ist. Price und Littlewood entwickelten dieses Projekt im Großbritannien der Nachkriegszeit und reagierten auf die instabilen sozio-ökonomischen Bedingungen. Diese Architektur der Mehrdeutigkeit oder Unbestimmtheit sollte eine Alternative zum konventionellen Freizeitangebot oder zu üblichen Bildungsansätzen bieten.

PK: Gab es eine Bedienungsanleitung dazu? Eine Bedienungsanleitung für die Unbestimmtheit?

4 Peter Koval bezieht sich hier explizit auf den Umbau einer Etage im Gebäude des Exzellenzclusters Bild Wissen Gestaltung in der Sophienstraße 22a, Berlin, zur sogenannten Experimentalzone. 
SH: Das weiß ich nicht. Es wurde vieles angedacht, aber inwieweit den Nutzer_innen tatsächlich Bedienungsanleitungen in die Hand gegeben werden sollten, ist mir nicht bekannt.

PK: Ich habe hier ein

12:00 min.

Wir verständigten uns auf ein Format, um die Charaktere der Kolaboranten interdisziplinär zu schärfen.

inzwischen älteres Projekt aus dem Musikinstrumentenbau oder eher Interface Design. Reactable (B 10) ist eine Installation, ein Musikinstrument, welches kollaborativ gespielt werden kann. Die Menschen betreten einen Raum und - ähnlich wie bei Fun Palace - fangen sie an, mit realen Objekten im Raum auf dem Tisch zu spielen. Und sie müssen spielen, sonst passiert ja gar nichts und sonst kann man auch nicht wissen, was in dem Raum alles passieren kann ...

$\mathrm{SH}$ : Ja, ich glaube, es ist sehr ähnlich. Beides muss man sich aneignen.

PK: Ja. Obwohl die Aneignungsschwelle bei Reactable recht niedrig ist, denn man hat das unmittelbare Feedback. Das Besondere daran ist für mich aber, dass man hier nicht im herkömmlichen Sinne auf einem Instrument spielt. Wenn ich den Raum als Instrument begreife, dann will

\section{3:00 min.}

Sie verfolgen gerade eine Verhandlung von vier unterschiedlichen Kolaboranten. Sie sollen uns als Modelle für die Wechselbeziehung von Raum und Bewegung dienen.

ich mit ihm bestimmte Sachen machen; bei einem Musikinstrument ist es eben die Musik. Doch das Interface von Reactable - die Gegenstände auf dem Tisch - ermöglicht nicht nur das Spielen auf dem Instrument, sondern zugleich auch das Spielen mit dem Instrument, die Manipulation seiner Struktur. Das Instrument selbst kann während des Spiels radikal verändert werden.

SH: Ich habe noch ein zweites Projekt, einen zweiten Mitspieler, mitgebracht, eigentlich nur, weil ich darüber gestolpert bin und mir dachte, ich muss es dir zeigen. Ein Projekt, das mich immer wieder fasziniert, und zwar das Blur Building von Diller \& Scofidio, welches sie für die Expo in der Schweiz 2002 entwickelten (B 11). Es ist ein Stahlgerüst, das sie auf dem See... nicht dem Bielersee, sondern...

PK: Du meinst den Neuenburgersee? 
SH: Ja, genau.

14:00 min.

Die runden Kärtchen stellen jeweils einen Kolaboranten dar, um den sich im Gesprächsverlauf vielfältige Wissensbezüge auf eckigen Bildkärtchen (an)sammeln.

Es besteht also aus einem begehbaren Stahlgerüst, das über unzählige kleine Düsen Seewasser verdampft oder eher versprüht und hierdurch eine Wolke erzeugt. Je nach Wetterlage breitet sich diese Wolke über dem Neuenburgersee aus.

Weshalb ich dieses Projekt erwähne? Ich habe ein zutreffendes Zitat mitgebracht. Philip Ursprung, der sich ja gleichermaßen in der Kunstgeschichte wie in der Architektur auskennt, schreibt in den Kritischen Berichten zum Blur Building Folgendes: »In der Wolke ist der Raum keine leere Bühne für ein Geschehen, kein neutrales Medium, in dem die Handlung abläuft, sondern vielmehr ein undurchsichtiger Akteur und Mitspieler. $\ll^{5}$

PK: Jetzt, wo ich es sehe, fällt mir wieder eine Analogie auf. Ich habe leider einen anderen wasserdampfenden Kolaboranten zu Hause gelassen.

\section{5:00 $\mathrm{min}$.}

Mit jedem Durchlauf gewinnen unsere Kolaboranten an Kontur, bleiben aber grundsätzlich in Bewegung.

Eine übliche Kaffeemaschine - das wissen vermutlich die wenigsten - kann man auch gut zum Kochen verwenden, und zwar als Dampfgarer. Die Temperatur des Wasserdampfes in einer Kaffeemaschine ist zwar nicht allzu hoch, reicht aber zum Garen von Lachs oder Brokkoli aus. Man muss es versucht haben, damit man es glaubt.

Aber lass uns jetzt zum letzten Kolaboranten kommen, nämlich zum Verrückten. Wir hatten lange überlegt, ob der Verrückte das richtige Wort ist, ob es um das Verrücken geht; oder ist es eher der Idiot, den wir meinen. Ich habe hier eine ganze Serie an Beispielen, die eine gewisse Kontinuität, vielleicht auch historische Kontinuität, aufweisen.

Zunächst eine Zeichnung von Rube Goldberg (B 12). Das war ein Zeichner, der in der ersten Hälfte des 20. Jahrhunderts regelmäßig Comics in US-amerikanischen Zeitungen

\section{6:00 min.}

Auf unserem Tisch liegen jetzt: allwissend schaltender Dirigent, offen vermittelnder Händler, interaktiv datenverarbeitender Mitspieler und störend entautomatisierender Verrückter. 
publiziert hat. Man sieht darauf immer eine über- oder zumindest verkomplizierte Apparatur, die als solche, wenn man sie experimentell im realen Raum aufstellen würde, vermutlich nicht funktionieren würde. Einige Künstler haben es übrigens trotzdem versucht und waren damit teilweise erfolgreich. Ob sie real funktionieren oder nicht, ist aber nicht von Belang - wir können sie uns im Kopf als Modelle vorstellen. Wir sehen hier eine äußerst komplizierte Apparatur, nur um den Brief des Herren aus seiner Brieftasche in den Briefkasten zu befördern. Eine durchaus analytisch-ironische Haltung zu dieser Art der Verrücktheit.

In den 1990er-Jahren wurde ein Computerspiel namens Incredible Machines auf den Markt gebracht. Dabei ging es nicht mehr, wie bei Goldberg, um kritische Analyse oder Ironie, sondern der Bau genau solcher >verrückten< Maschinen wurde zum pädagogischen Ziel erklärt. Das ist ein Spiel, welches

\section{7:00 min.}

Sie bilden eine sonderbare Struktur, die eben kein Abbild eines bestimmten Wissensgebietes ist, sondern eine mit jedem Durchlauf neu gezeichnete wissensarchitektonische Karte.

zukünftige Physiker spielen sollten, um sich rechengestützte Modellierungen von physikalischen Experimenten auf spielerische Art und Weise anzueignen oder zumindest vorzustellen.

Doch den größten Idioten konnte ich in der Uhrenindustrie ausfindig machen. Im Uhrenbau gibt es die sogenannten Komplikationen. Eine Uhr zeigt die Uhrzeit an. Alles, was darüber hinaus geht, ist im Grunde eine gewollte mechanische Komplikation. Ob ewiger Kalender, Minutenrepetition oder 3D-Turbillon: Eigentlich braucht man so etwas in Zeiten von Smartwatches nicht. Für Uhrenmechaniker_innen sind aber solche >Umständlichkeiten< die größten Herausforderungen, und gerade sie - ihre Verrücktheit - bringen die Höchstleistungen der Feinmechanik hervor.

SH: Aber gibt es hier wirklich für die Nutzenden noch Denkanstöße?

PK: Dazu müsste man sie tatsächlich befragen.

18:00 $\min$.

Diese Karte gibt der interdisziplinären Aushandlung eine konkrete Form und dokumentiert zugleich ihren Entstehungsprozess.

SH: Ich habe lange über den Verrückten oder Idioten nachgedacht. Er ist derjenige, der entrückt, verlangsamt, Denkanstöße bietet - und da gibt es natürlich viele Alltagssituationen in der Architektur, denen man solche >Verrücktheiten< nachsagen kann. Teilweise auch solche, wo vielleicht die Architekt_innen versagt haben. 
Ein Projekt, oder ein Verrückter, ist das Reversible Destiny Loft von Arakawa und Madeline Gins (B 13), einem japanisch-amerikanischen Paar. Ihre Theorie des Reversible Destiny entwickelten sie über Jahrzehnte in Abhandlungen und Büchern, in Kunstwerken und schließlich auch in der Architektur. Ihr erklärtes Ziel war, dem Tod zu trotzen - beide sind leider inzwischen verstorben. Dieses Apartmenthaus befindet sich im Randbereich von Tokio. Nicht nur von außen, auch im Inneren des Hauses ist nichts so, wie man es eigentlich von einem Wohngebäude erwartet: Die Lichtschalter

\section{9:00 min.}

Die Kolaboranten verkörpern eine bestimmte Beziehung zwischen Raum und Bewegung. Sie bestimmen aktiv den weiteren Entwurf und leiten das architektonische Experimental-Setting ein.

liegen nicht auf Griffhöhe neben den Türen, die Durchgänge zu den Terrassen sind kleine Öffnungen, durch die man hindurchkriechen muss, die Böden sind uneben ... Überall befinden sich Hindernisse. Man könnte nun sagen, dass diese Stolpersteine und Denkanstöße eben im alltäglichen Gebrauch schnell wieder verloren gehen. Doch haben Arakawa und Madeline Gins für die Benutzung des Gebäudes Anleitungen entwickelt, wie man jeden Tag seine Wohnung neu erlernen kann.

PK: Und wohnt auch jemand freiwillig in diesem Haus? Es sind auf dem Bild nur leere Räume zu sehen.

$\mathrm{SH}$ : Ja, es ist bewohnt, und ich konnte eine Wohnung besuchen.

PK: Und lernen die Bewohner_innen die Regeln tatsächlich? Oder hängt die Anleitung an der Wand wie eine Kalligrafie in einer Tokonoma? ${ }^{6}$

SH: Es hängt wohl von den Ambitionen der jeweiligen Bewohner_innen ab - davon, wie ernst sie es mit dem Leben nehmen.

\section{0:00 min.}

Vielen Dank für Ihre Aufmerksamkeit!

6 Tokonoma ist eine Zimmernische in der traditionellen japanischen Architektur, in der meist Kalligrafien oder Bildrollen sowie Blumengestecke zu dekorativen bzw. kontemplativen Zwecken präsentiert werden. 


\section{Literatur}

Joerges, Bernward (1996): Technik - Körper der Gesellschaft: Arbeiten zur Techniksoziologie. Frankfurt a. M.:

Suhrkamp.

Ursprung, Philip (2001): Weisses Rauschen: Elisabeth Diller und Richard Scofidios Blur Building und die räumliche Logik der jüngsten Architektur. In: Kritische Berichte - Zeitschrift für Kunst- und Kulturwissenschaften, Jg. 29, Nr. 3, S. 5-15.

Graßhoff, Gerd (2014): Kollaborative Autorschaft in den Wissenschaften. Vortrag im Rahmen der Ringvorlesung Interferenzen der et al ${ }^{\circ}$ - Vereinigung Wissenschaft, Kultur und Medizin. Tieranatomisches Theater der HumboldtUniversität zu Berlin, 15. Mai.

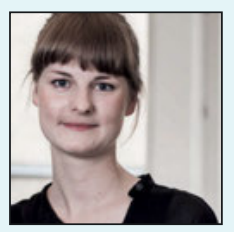

sabine.hansmann@hu-berlin.de

Wissenschaftliche Mitarbeiterin

Basisprojekte: Gestaltung von Laboren, Mobilität

Disziplin: Architektur

Sabine Hansmann hat Architektur und Design studiert. Als wissenschaftliche Mitarbeiterin im Exzellenzcluster Bild Wissen Gestaltung untersucht sie offene und dynamische Raumstrukturen und Prozesse, die über das architektonische Objekt hinausweisen.

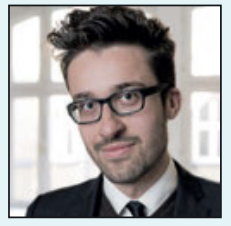

peter.koval@hu-berlin.de

Wissenschaftlicher Mitarbeiter

Basisprojekt: Experiment \& Beobachtung

Disziplin: Kulturwissenschaft

Peter Koval hat in Kulturwissenschaften promoviert. Als PostDoc am Exzellencluster Bild Wissen Gestaltung beschäftigt er sich mit der Wirksamkeit von bewusst gestalteten (Erkenntnis-)Hindernissen. 


\section{Christian Stein}

\section{Im Umkreisen begriffen}

\section{Über die Produktivität kommunikativer Ambiguität}

»Die Sprache ist aber durchaus kein bloßes Verständigungsmittel, sondern der Abdruck des Geistes und der Weltsicht des Redenden.«

Wilhelm von Humboldt, Über den Dualis, 1827

Immer, wenn wir über Wissen reden - wie im Namen des Interdisziplinären Labors Bild Wissen Gestaltung -, immer, wenn wir etwas sagen oder hören, sehen oder zeigen, ausprobieren oder beherrschen, immer dann geht es um das Verstehen. In Kommunikations- und Kollaborationssituationen wie dem Interdisziplinären Labor kommt die Frage nach dem Verstehen in unterschiedlicher Gestalt immer wieder auf. Sie ist essenziell in dem Sinne, als dass in der Vereinigung sehr heterogener Qualifikationen und Perspektiven spürbar wird, was sonst leicht in Vergessenheit geraten kann: Verstehen ist keine Selbstverständlichkeit. Kommunikation kann andauern, ohne dass Verständnis erzeugt wird. Der Glaube und die Beteuerung zu Verstehen erzeugen es noch nicht. So werden gerade in der interdisziplinären Kooperation das Missverstehen, das Aneinander-Vorbeireden, das Unverständnis, der Rückzug auf das eigene Fachgebiet und die heterogene Prioritätensetzung sehr deutlich sichtbar und als Schwierigkeit empfunden. Diese Problematisierung, die sich immer wieder in Diskussionen niederschlägt, ist aber auch eine große Chance, sie produktiv zu wenden. 


\section{Aneignung einer Sprache}

Um der Frage nach der Funktionsweise einer spezifischen wissenschaftlichen Fachsprache nachzugehen, ist es sinnvoll, sich deren Adaption vor Augen zu führen. Ähnlich wie beim Erlernen einer Fremdsprache geht auch dem Fachsprachenerwerb eine bereits bestehende Ausgangssprache voraus. Wissenschaftler_innen waren nicht immer Wissenschaftler_innen. Vor dem sprachlichen und intellektuellen Eintritt in das System Wissenschaft waren sie einfach Menschen, die gelernt haben, sich für verschiedene Bereiche zu interessieren. Typischerweise fokussiert sich dieses Interesse ab einem bestimmten Entwicklungspunkt auf einige Themenkreise, um mehr Zeit in diese investieren zu können. Die Auseinandersetzung mit diesen Themen erfolgt zunächst von einem neutralen Punkt aus, der weder vom wissenschaftlichen Diskurs noch von dessen Sprache geprägt ist. Dementsprechend erfolgt der sprachliche und konzeptuelle Zugang auch über die Gemeinsprache, die zwar nicht die terminologische Präzision einer Fachsprache aufweist, dafür aber auf alle Betrachtungsgegenstände anwendbar ist.

Disziplin: Teil einer historisch gewachsenen Einteilung von Wissenschaftsgebieten, der institutionell verankert ist und eigene Diskurse entwickelt, zum Beispiel Mathematik.

Signifikant: materielle, sinnlich wahrnehmbare Ausdrucksseite eines Zeichens im Sinne von dem, was bezeichnet, zum Beispiel Buchstaben- oder Lautfolge $<$ Baum $>$ bzw. [bAUm].

Signifikat: Inhaltsseite eines Zeichens im Sinne von Vorstellungsbild, Begriff oder Bedeutung, auf die ein Signifikant verweisen kann, zum Beispiel >Vorstellung eines Baumes<.

Terminus: vollständiges sprachliches Zeichen innerhalb einer Fachsprache, bestehend aus Signifikant und Signifikat, zum Beispiel >Transterminologisierung<(siehe unten). ${ }^{1}$

Mit dem Eintritt in eine wissenschaftliche Disziplin ändert sich dieser neutrale Zugang. Der interessierte Mensch wird zum Wissenschaftler oder zur Wissenschaftlerin und lernt im Zuge dessen, die zuvor ins Auge gefassten Gegenstände professionell zu betrachten, zu konzeptualisieren und zu benennen. Dieser disziplinäre Zugang ist mit einem Umdenken verbunden, das in der Folge die gemeinsprachlichen Zugänge als naiv erscheinen lässt und nach und nach durch die entsprechende Fachsprache ersetzt (Abb. 1). Dies betrifft nicht nur die Signifikanten, sondern vor allem auch die Signifikate, also die Bedeutungsebene. Man kann sagen, dass die Sprache und die Perspektive gleichermaßen > diszipliniert< werden. Diese Disziplinierung ist anstrengend, da das Interesse allein nun nicht mehr ausreicht, um sich mit einem Gebiet auseinanderzusetzen - auch die richtige Sprache ist jetzt wichtig. Die so entstehende Hürde,

1 Die hier gegebenen Definitionen sind selbst formuliert, spiegeln aber gängige Definitionen der Linguistik wieder. $<$ Terminus> ist dahingehend ein Sonderfall, als dass die Terminologienorm DIN 2342 in der neuen revidierten Version von $2011<$ Terminus> umdefiniert hat als Synonym zu Benennung. Dieser Auffassung wird hier nicht gefolgt. 
Taxonomie: Nach einem definierten Klassifikationsschema hierarchisch geordnete Sammlung von Termini, die ein formales Sprachsystem bilden, zum Beispiel Tierarten.

Ontologie: Formale Modellierung einer Menge von Termini mit verschiedenen semantischen Relationen zwischen diesen, die ein Bedeutungsnetzwerk bilden, zum Beispiel SKOS (Simple Knowledge Organization System).

\section{Verfestigung der Struktur}

Nach einiger Zeit hat der Wissenschaftler einen signifikanten Teil der Termini seiner Disziplin und deren Verbindungen erlernt und verfügt über ein stabiles, aktives Netzwerk. Wichtig ist dabei, dass Sprache nicht nur der Kommunikation dient - sie dient auch dem Verstehen: Welche Elemente als Einheiten aufgefasst werden, wo Differenzen gezogen werden, was etwas bedeutet, womit etwas zusammenhängt - all das liegt zu einem großen Teil nicht in den Betrachtungsgegenständen selbst, sondern im Wissen der Disziplinen. Dementsprechend verwundert es nicht, dass sich dieses Wissen kontinuierlich ändert und dass alle großen Paradigmenwechsel sich immer auch in einer veränderten Terminologie niederschlagen.

\section{— Die eigentliche Bedeutung liegt im Netzwerk, nicht in seinen Elementen.}

Das sich stabilisierende terminologische Netzwerk der angehenden Wissenschaftlerin charakterisiert demnach nicht nur ihre Art zu sprechen und zu schreiben, sondern auch ihre Art zu denken: Es ist ein Wissensnetzwerk. Da alles mit allem vermascht ist, lassen sich Aspekte dieses Wissensnetzwerks nicht mehr ohne Weiteres austauschen. Ludwik Fleck nennt diesen Effekt »Beharrungstendenz ${ }^{4}$ und verweist damit darauf, dass das Wissen einer Disziplin aus der Innenperspektive der Denkkollektive als so selbstverständlich erscheint, dass eine denkstrukturelle Veränderung nur sehr langsam möglich ist:

\footnotetext{
»Nicht um bloße Trägheit handelt es sich oder Vorsicht vor Neuerungen, sondern um eine aktive Vorgehensweise, die in einige Grade zerfällt: 1. Ein Widerspruch gegen das System erscheint undenkbar 2. Was in das System nicht hineinpaßt, bleibt ungesehen, oder 3. es wird verschwiegen, auch wenn es bekannt ist, oder 4. es wird mittels großer Kraftanstrengung dem Systeme nicht widersprechend erklärt. 5. Man sieht, beschreibt und bildet sogar Sachverhalte ab, die den herrschenden Anschauungen entsprechen, d.h. die sozusagen ihre Realisierung sind - trotz aller Rechte widersprechender Anschauungen. $\ll^{5}$
}

4 Fleck 1980, 141.

5 Ebd., 40. 


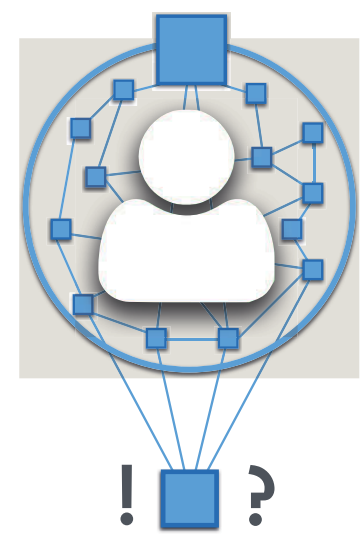

Abb.3: Integration neuer Gegenstände in ein etabliertes disziplinäres Wissensnetzwerk.

Dieser strukturellen Widerständigkeit steht eine inhaltliche Offenheit gegenüber. Neue Themen und Forschungsgegenstände tauchen nicht nur auf, sie werden auch aktiv gesucht.

Das Wissensnetzwerk des Forschers ist darauf ausgerichtet, neue Inhalte zu erfassen und mit dem bestehenden Wissen zu verknüpfen (Abb.3). Diese Verknüpfungen transformieren den unbestimmten Status des Neuen - was auch immer es sein mag - zügig in den bestimmten Status des Anwendungsfalls. Je umfangreicher und stabiler ein Wissensnetzwerk ist, umso schneller kann der Wissenschaftler Verknüpfungen aufbauen und umso schwerer fällt es ihm, eine vorläufig unbestimmte Betrachtungshaltung beizubehalten. Das Neue ist schnell nicht mehr neu, sondern stabilisierender Teil der Struktur geworden.

\section{— Wissensnetzwerke weisen strukturelle Widerständigkeit bei inhaltlicher Offenheit auf.}

Gelegentlich nun kommt es vor, dass ein Gegenstand im Blick der Wissenschaftlerin auftaucht, der sich der Integration in das Wissensnetzwerk hartnäckig widersetzt. In einem solchen Fall sind verschiedene Reaktionen möglich: Der Wissenschaftler kann negieren, dass das Objekt als Forschungsobjekt überhaupt existiert bzw. relevant ist, und das Problem somit lösen. Er kann das Objekt jedoch auch umdefinieren, einer spezifischen Klasse zuordnen oder eine Analogie herstellen und so über einen Zwischenschritt eine Anschlussfähigkeit generieren. Oder er ordnet das Objekt einem weit entfernten Bereich zu, der keine Bezugspunkte zu seinem disziplinären Wissen aufweist und schiebt es damit einer anderen Disziplin zu. In jedem Fall muss er mit dem Objekt umgehen, da eine bleibende Inkonsistenz mit seinem bestehenden Wissensnetzwerk eine strukturelle Veränderung dessen erforderlich machen würde. Ab einem gewissen Stabilitätsgrad weist das Wissensnetzwerk jedoch eine eigene Resilienz auf, eine Widerständigkeit und Fähigkeit zur Selbsterhaltung, die eine Veränderung nur dann zulässt, wenn sich das problematische Objekt nicht auflösen lässt. 


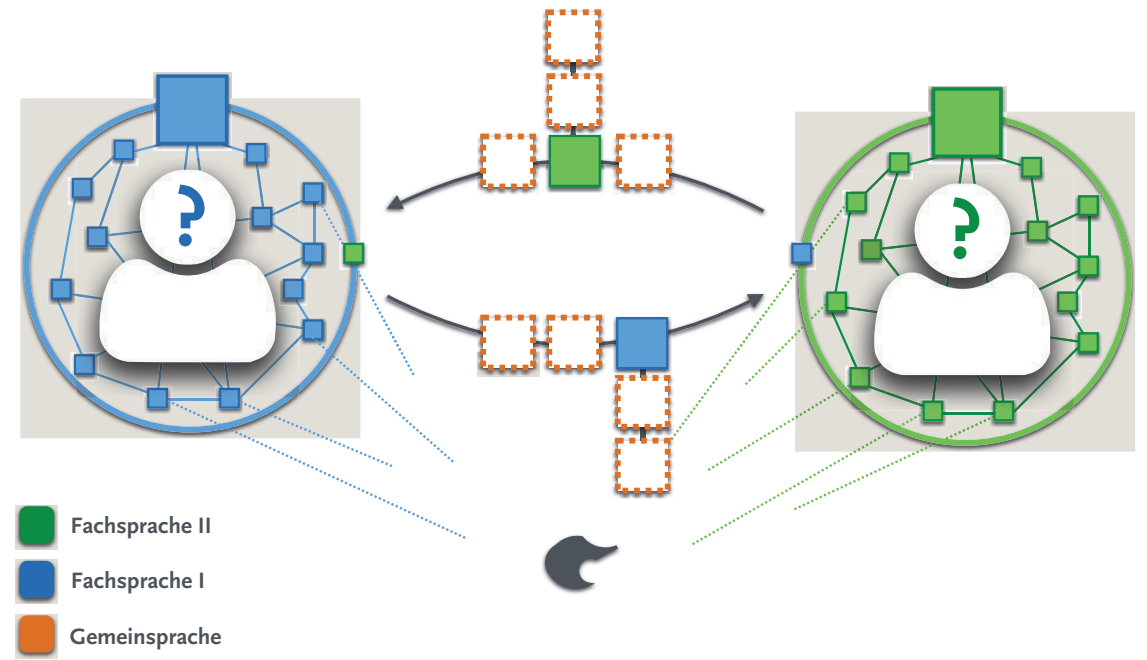

Abb.4: Interdisziplinäre Kommunikation unter Zuhilfename der Gemeinsprache.

\section{Versuch einer Kommunikation}

Dadurch, dass die Wissenschaftlerin ihr Wissensnetzwerk in ihrer Disziplin erlernt und ausgebaut hat, fällt ihr die Kommunikation innerhalb dieser und mit nahegelagerten verhältnismäßig leicht. Anders wird das, wenn es um die Kommunikation mit einem Wissenschaftler aus einer denkstrukturell weiter entfernten Disziplin geht, der sein ganz eigenes und fundamental anderes Wissensnetzwerk besitzt. In interdisziplinären Großprojekten, wie dem Exzellenzcluster Bild Wissen Gestaltung, kommt es ständig zu einem solchen Aufeinandertreffen (Abb.4). Wenn die beiden Wissenschaftler_innen nun auch noch am gleichen Forschungsgegenstand und zusammen arbeiten sollen oder wollen, müssen sie eine geeignete Kommunikationsform finden. Ihre Wissensnetzwerke sind dabei genauso inkompatibel wie ihre jeweilige Terminologie, sodass sie nicht so miteinander sprechen können wie mit einer Kollegin oder einem Kollegen ihrer Disziplin: »Echte Fachsprache ist immer an den Fachmann gebunden, weil sie volle Klarheit über Begriffe und Aussagen verlangt. Vom Nichtfachmann gebraucht, verliert die Fachsprache ihre unmittelbare Bindung an das fachliche Denken. ${ }^{6}$

Gleichzeitig sind beide Wissenschaftler_innen in ihrer fachlichen Kompetenz gefragt und wollen wissenschaftlich arbeiten, was die gemeinsame Rückfallebene der Gemeinsprache mit ihrer Ambiguität zunächst ungeeignet erscheinen lässt. Typischerweise werden sie daher versuchen, die Gemeinsprache überall dort einzusetzen, wo die Ambiguitäten in Bezug auf den spezifischen gemeinsamen Forschungsgegenstand verschmerzbar sind, und fachspezifische Termini dort

6 Hoffmann 1976, 31. 
einzuführen, wo sie einen bedeutenden Unterschied und eine wichtige Perspektive markieren (Abb. 5). Sie werden versuchen, dem jeweils anderen Wissenschaftler diese Termini gemeinsprachlich zu definieren, sodass dieser sie fortan verstehen und gegebenenfalls sogar selbst verwenden kann. Dieses Bemühen um ein gemeinsames Verständnis ändert jedoch nichts daran, dass die jeweils neu gelernten Termini den Wissenschaftler_innen äußerlich bleiben. Sie verfügen zwar über ein rudimentäres Verständnis, nicht jedoch über die Integration in ein stabiles Wissensnetzwerk. Es ist eine dekontextualisierte Terminologie, die eher auf der Ebene von Vokabellernen denn von Anwendungswissen rangiert.

Interdisziplinarität: Zusammenführen verschiedener Perspektiven, Methoden und Werkzeuge aus unterschiedlichen Disziplinen zu etwas Neuem.

Multidisziplinarität: Nebenläufige Bearbeitung einer Fragestellung durch verschiedene Disziplinen ohne nennenswerten Austausch zwischen diesen.

Transdisziplinarität: Problemzentrierte Bearbeitung einer Frage durch verschiedene Disziplinen, bei der Wissen von einer Disziplin in einer anderen übernommen wird.

Bereits auf diesem Weg müssen die Wissenschaftler_innen die Bereitschaft erworben haben, die perspektivischen und terminologischen Konflikte ihrer Wissensnetzwerke nicht nur auszuhalten, sondern an einer echten Kommunikation zu arbeiten - es versteht sich dabei von selbst, dass Interdisziplinarität bereits hier leicht scheitern kann und dies auch häufig tut. Was als Interdisziplinarität initiiert war, erweist sich dann als Multidisziplinarität, in der die Wissenschaftler_innen ihre Berührungspunkte auf ein konflikt-, aber auch kommunikationsarmes Minimum reduzieren und im Wesentlichen nebeneinanderher arbeiten. Die Produktionsgeschwindigkeit wird dadurch signifikant erhöht - das oftmals erhoffte Innovationspotenzial interdisziplinärer Projekte bleibt so jedoch notwendigerweise aus. ${ }^{7}$

\section{Produktive Ambiguität}

Ist die anfänglich stockende und immer noch nicht einfache Kommunikation zwischen den beiden Wissenschaftler_innen erfolgreich angelaufen und konnte sich rudimentär stabilisieren, kommt es in vielen Fällen zu einem interessanten kommunikativen Phänomen. Die Wissenschaftler_innen stolpern über einen Signifikanten, der in ihren beiden, heterogenen Fachsprachen vorkommt. Sie bemerken diese Überschneidung und realisieren schnell, dass sich die Signifikate bzw. Bedeutungen innerhalb ihrer jeweiligen Disziplin unterscheiden. Interessant wird es dann, wenn die entsprechenden Termini in beiden Wissensnetzwerken eine wichtige Position einnehmen - mit jeweils unterschiedlicher Bedeutung und Vernetzung. Häufig handelt es sich

7 Vgl. z. B. Klein 2012, 15. 


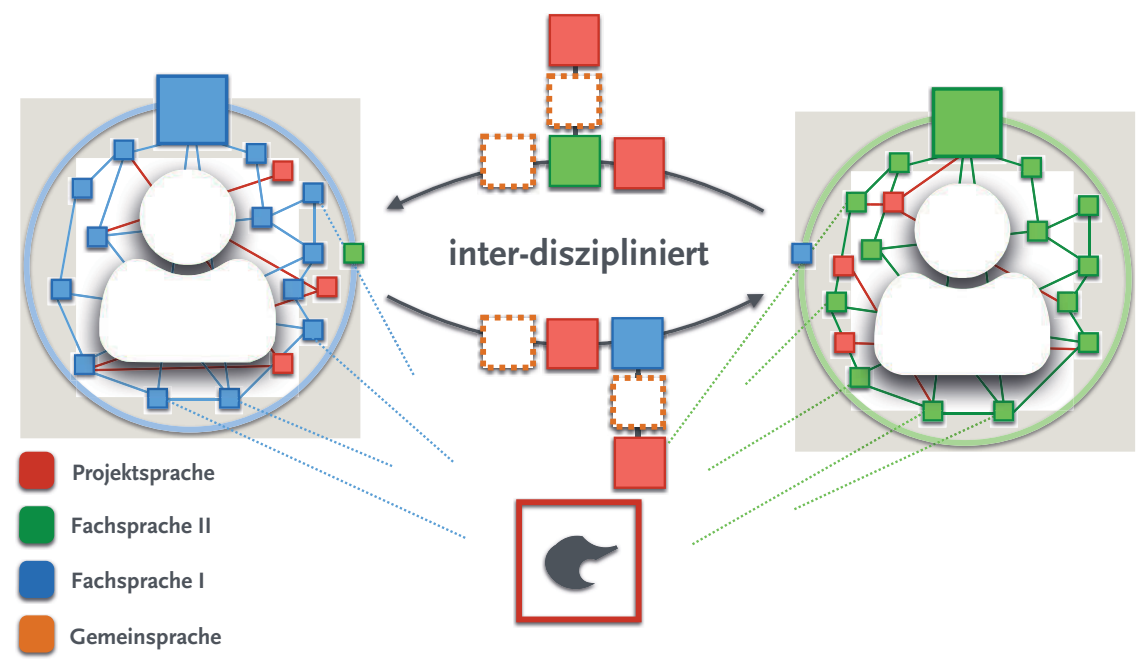

Abb. 5: Interdisziplinäre Kommunikation unter Verwendung einer Projektsprache.

dabei dann um Terminologisierungen bzw. Transterminologisierungen, die in vielen Disziplinen vorgekommen sind. Durch den gemeinsamen etymologischen Ursprung stellen diese synchron häufig Polyseme dar, die einen gemeinsamen Bedeutungskern aufweisen.

Terminologisierung: Übertragung eines gemeinsprachlichen Zeichens in eine Fachsprache unter Angabe einer präzisen fachspezifischen Definition.

Transterminologisierung: Übertragung eines fachsprachlichen Zeichens in eine andere Fachsprache unter Angabe einer veränderten Definition.

Etymologie: Wortherkunft bzw. Veränderung eines sprachlichen Zeichens über die Zeit, sowoh auf Signifikat- wie auch auf Signifikantebene.

Synchron: Eigenschaften einer Sprache zu einem Zeitpunkt mit Fokus auf die Beziehungen der Sprachzeichen zueinander und nicht ihrer etymologischen Entstehung.

Einen solchen Signifikanten, der in vielen Fachsprachen mit unterschiedlichen, aber verwandten Bedeutungen vorkommt, nenne ich Interterminus. Intertermini haben einige besondere Eigenschaften, die sie von herkömmlichen Termini unterscheiden:

> Sie sind polysem.

> Sie kommen in verschiedenen Disziplinen vor.

> Sie sind etymologisch verwandt.

> Sie treten in den Terminologiegebäuden der jeweiligen Disziplinen relativ abstrakt und stark vernetzt auf. 
> Sie lassen sich nicht einfach ersetzen oder umbenennen.

> Sie kommen häufig auch in der Gemeinsprache vor, wo sie in breiten Verwendungszusammenhängen benutzt werden.

> In gemeinsprachlicher Verwendung findet sich häufig auch ein metaphorischer Gebrauch.

In der Kommunikation der zwei Wissenschaftler_innen haben all diese Eigenschaften von Intertermini meist zur Folge, dass die beiden sich nicht schnell auf eine Definition einigen können. Sie sind aber auch nicht bereit, einfach eine solche zu akzeptieren, da der Signifikant in ihren beiden Systemen eine wichtige Position einnimmt. Aus diesen Situationen ergeben sich die spannendsten Diskussionen. Häufig wird dann versucht, eine Definition zu finden, die beide fachspezifische Signifikate inkludiert. Das Ziel ist es somit, ein neues, übergeordnetes Signifikat zu konstruieren, das die beiden disziplinären Signifikate einschließt und das ebenfalls den gleichen Signifikanten aufweist (Abb.6).

\section{- Beobachtete Beispiele für Intertermini aus dem Interdisziplinären Labor: Architektur, Bild, Wissen, System, Struktur, Modell, Akteur_in.}

Das Besondere daran ist, dass die um Intertermini entstehenden Diskussionen eigentlich auf einem Irrtum beruhen: Die Wissenschaftler_innen gehen davon aus, dass die beiden Signifikate ihrer jeweiligen Fachsprache eine gemeinsame, übergeordnete Definition aufweisen - weil sie den gleichen Signifikanten aufweisen und beide ihre Gültigkeit behaupten. In Wirklichkeit ist das nicht der Fall. Die Etymologie ist für eine synchrone Betrachtung nicht notwendigerweise relevant; den Ursprung eines Wortes zu kennen mag zwar interessant sein, spielt bei der Bestimmung seiner Verwendung in der aktuellen Sprache aber keine wesentliche Rolle. Analog dazu gibt es nicht notwendigerweise einen abstrakten gemeinsamen Oberbegriff, der die beiden Signifikate vereinen würde - häufig ergibt sich ein solcher erst aus der gefühlten Notwendigkeit, welche die Diskussion um einen Interterminus hervorruft. Der logos in >Terminologie< verweist in diesem Zusammenhang auf die Annahme eines geordneten und auch vollständigen Begriffsnetzwerkes.

Die Definitionen, die bei der diskursiven Arbeit um einen Interterminus implizit oder explizit entstehen, müssen notwendigerweise so abstrakt sein, dass sie in den einzelnen Disziplinen oder der Praxis kaum noch eine terminologische Funktion ausüben, da sie keine relevanten Differenzen erzeugen. Ihre Funktion ist eine ganz andere. Sie sind nicht dazu geeignet, in Diskussionen über bestimmte Aspekte des Forschungsgegenstands selbst verwendet zu werden, sondern dazu, einen gemeinsamen sprachlichen Boden für gelingende interdisziplinäre Forschung zu bereiten. 


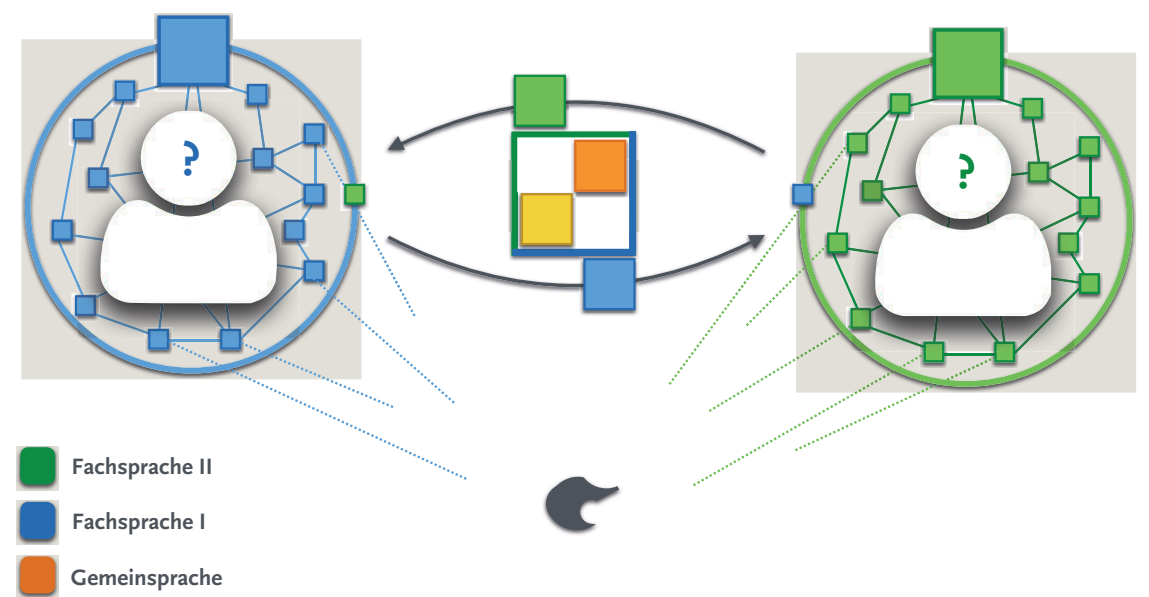

Abb. 6: Entdeckung eines Interterminus in der Diskussion.

\section{Gemeinsamer Boden}

Intertermini wirken als kommunikative Brücke. Wissenschaftler_innen erhalten so die Möglichkeit, weitere Signifikanten und Signifikate mit den Intertermini zu verknüpfen und so eine eigene Projektsprache aufzubauen. Eine solche Projektsprache besteht jedoch nur zu einem kleinen Teil aus Intertermini. Der umfangreichere Teil ergibt sich direkt aus dem Forschungsgegenstand und muss daher deutlich konkreter sein. Die Kernanforderung an eine Terminologie besteht im Wesentlichen darin, über den Beschreibungsgegenstand differenziert und eindeutig kommunizieren zu können. Es geht nicht darum, ihn in ein bestehendes Klassifikationssystem einzuordnen, sondern vielmehr, die notwendigen Differenzen am Gegenstand selbst zu entwickeln und sprachlich eindeutig zu codieren. ${ }^{8}$

Eine gut funktionierende Projektsprache geht damit den umgekehrten Weg: Nicht primär die Wissensnetzwerke der Wissenschaftler_innen sind es, zu denen Relationen aufgebaut werden; vielmehr gehen die Terminologiebedarfe vom Gegenstand selbst aus. Wo sich in den Wissensnetzwerken dazu geeignete Termini finden, werden diese genutzt - wo nicht, neue definiert.

Die Projektsprache hat damit zwei wesentliche Orientierungspunkte, die beide nicht die bestehenden Wissensnetzwerke der Wissenschaftler_innen sind: Den oder die zentralen, abstrakten und in der Diskursion konstruierten Interterminus bzw. Intertermini und die sich aus dem Forschungsgegenstand selbst ergebenden konkreten Projekttermini. Eine solche Projektsprache ist dabei notwendigerweise projektspezifisch und kann nicht einfach auf andere Projekte übertragen werden. Es ist gerade der Prozess des Aufbaus einer gemeinsamen Sprache, die weder im Wissensnetzwerk des einen noch des anderen Wissenschaftlers angesiedelt ist, der

8 Vgl. z. B. Frey 2010, 77-88. 
Kommunikation gelingen lässt. Ist die wechselseitig erlernte Terminologie des jeweils anderen vor dem Aufbau einer Projektsprache noch äußerlich und schlecht integriert, wird diese gerade durch den Akt ihrer aktiven Konstruktion zum gut eingebundenen und vernetzen Teil der jeweiligen Wissensnetzwerke. Eine solche Projektsprache erweitert nicht nur das Verstehen der Wissenschaftler_innen, sie ändert ihr Denken nachhaltig.

\section{— Terminologie ist territoriales Verhalten im Sprachraum.}

Das eigene Wissensnetzwerk mit der eigenen Terminologie ist gewissermaßen das Zuhause des Einzelnen oder der Disziplin im Sprachraum. Hier kennt man sich aus, fühlt sich wohl, findet sich blind zurecht, findet Zeichen für sich selbst überall wieder. Es ist aber auch ein Ort, an dem jeder Andere Gast ist. Ein zu aufdringlicher Gast ist genauso unwillkommen, wie er sich selbst unwohl fühlen wird. Dieses Verhältnis macht die interdisziplinäre Zusammenarbeit häufig so schwierig. Wird etwa ein typischerweise einer bestimmten Disziplin zugeordneter Forschungsgegenstand mit einer disziplinfremden Terminologie beschrieben, ist die Reaktion häufig totale Ablehnung - unabhängig von den ausgesagten Inhalten. Terminologie bildet damit eine sprachliche Selbstbehauptung, signalisiert eine Deutungshoheit und legt die Regeln dieses Bereichs fest: Terminologie ist territoriales Verhalten im Sprachraum. Ludwik Fleck schreibt:

»Worte, früher schlichte Benennungen, werden Schlagworte; Sätze, früher schlichte Feststellungen, werden Kampfrufe. Dies ändert vollständig ihren denksozialen Wert; sie erwerben magische Kraft, denn sie wirken geistig nicht mehr durch ihren logischen Sinn - ja, oft gegen ihn - sondern durch bloße Gegenwart. «9

Mit dem Aufbau einer gemeinsamen Projektsprache jedoch wird ein neuer Ort geschaffen. Es ist eine gemeinsame Raumnahme eines dann gemeinschaftlich bewohnten neutralen Orts. Dieser neue Ort vermeidet die territorialen Konflikte und bietet das Potenzial, sich voll und ganz auf den Forschungsgegenstand zu konzentrieren. Die Kommunikation im Projekt sieht danach anders aus: Sie besteht aus einem Mix von Gemeinsprache, Projektsprache und mittels dieser beiden definierter Fachsprache. Nach Beendigung des Projekts wird die Projektsprache in den Wissensnetzwerken der Wissenschaftler_innen verbleiben und künftige Projekte beeinflussen (Abb. 7).

9 Fleck 1980, 59. 


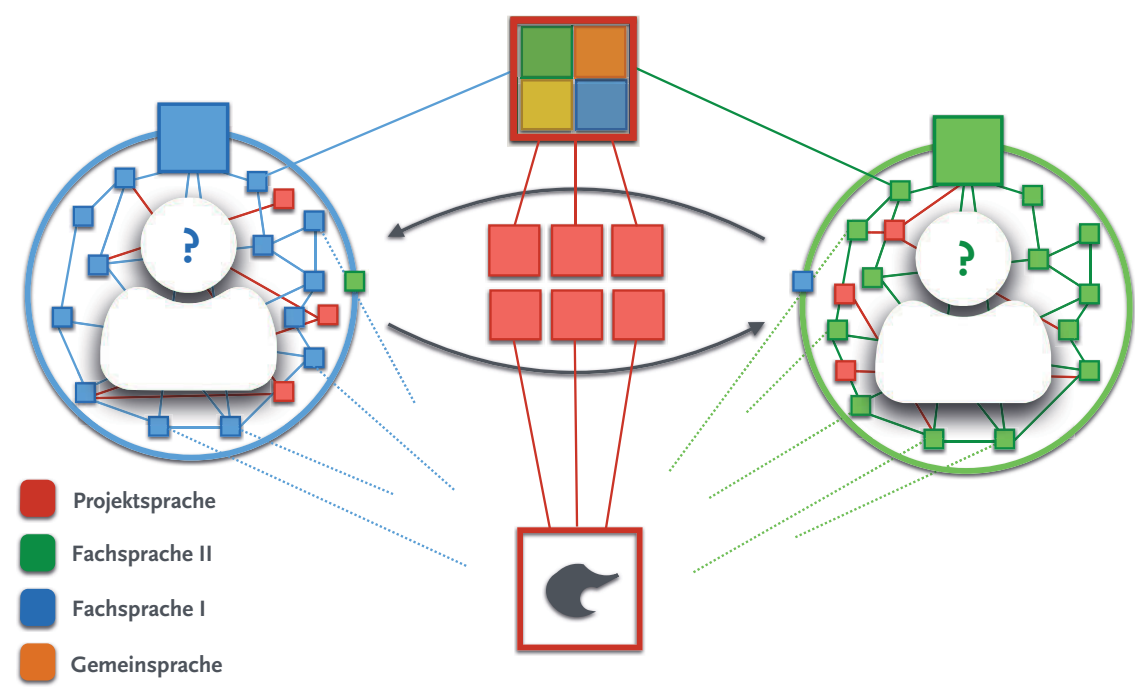

Abb. 7: Entstehung einer Projektsprache mit Intertermini und konkreten Gegenstandsbezügen.

\section{Interdisziplinarität als Umkreisen}

Die Wissenschaftlerin hat damit einen Prozess durchlaufen, an dessen Anfang die sprachliche Disziplinierung stand und an dessen Ende eine > Un-Disziplinierung< erreicht wurde. Dort, wo die Stabilität disziplinierter Fachsprache nicht mehr nur Halt und Sicherheit gibt, sondern in Unflexibilität bis hin zum Selbstzweck umschlägt, können die undisziplinierte Projektsprache und der überdisziplinäre Interterminus einen Ausweg bieten, der nicht in Opposition zu den bestehenden Wissensnetzwerken steht, sondern diese öffnet und erweitert. Interdisziplinarität heißt dort, wo sie gelingt, also auch, die eigene Disziplin mit einem Bein zu verlassen und sich darauf zu besinnen, wozu Terminologie eigentlich gebraucht wird: um verstanden zu werden und um zu verstehen.

Friedrich Nietzsche hat in seiner frühen sprachphilosophischen Schrift Über Wahrheit und Lüge im außermoralischen Sinne das Verhältnis des Wissenschaftlers zur Sprache als Kolumbarium beschrieben:

»An dem Bau der Begriffe arbeitet ursprünglich, wie wir sahen, die Sprache, in späteren Zeiten die Wissenschaft. Wie die Biene zugleich an den Zellen baut und die Zellen mit Honig füllt, so arbeitet die Wissenschaft unaufhaltsam an jenem großen Kolumbarium der Begriffe, der Begräbnisstätte der Anschauungen, baut immer neue und höhere Stockwerke, stützt, reinigt, erneut die alten Zellen und ist vor allem bemüht, jenes ins Ungeheure aufgetürmte Fachwerk zu füllen und die ganze empirische Welt, das heißt die anthropomorphische Welt, hineinzuordnen. $\ll^{10}$

10 Nietzsche 1873,886 . 
Ein Kolumbarium ist jedoch nicht nur eine Begräbnisstätte - ursprünglich ist damit der Taubenschlag gemeint. In diesem Sinne lässt sich auch wissenschaftliche Sprache im gelingenden interdisziplinären Diskurs umdeuten: Nicht in der Starrheit und Statik der Begiffssysteme liegt dann ihre Stärke, sondern im lebendigen, vielleicht chaotischen, sich umflatternden und umkreisenden Hinein-und-wieder-Hinaus einer Kommunikation, die sich ihre ganz eigenen dynamischen Verbindungen im Sprachraum geschaffen hat. 


\section{Literatur}

Budin, Gerhard (1996): Wissensorganisation und Terminologie: die Komplexität und Dynamik wissenschaftlicher Informations- und Kommunikationsprozesse. Tübingen: Gunter Narr Verlag.

Fleck, Ludwik (1980): Entstehung und Entwicklung einer wissenschaftlichen Tatsache. Einführung in die Lehre vom Denkstil und Denkkollektiv. Hg.v. Schäfer, Lothar/ Schnelle, Thomas. Frankfurt a. M.: Suhrkamp.

Frey, Ulrich (2010): Im Prinzip geht alles, ohne Empirie geht nichts - Interdisziplinarität in der Wissenschaftstheorie. In: Jungert, Michael u. a. (Hg.): Interdisziplinarität: Theorie, Praxis, Probleme. Darmstadt: Wissenschaftliche Buchgesellschaft, S. 77-88.

Hoffmann, Lothar (1976): Kommunikationsmittel Fachsprache. Berlin: Akademie-Verlag.
Humboldt, Wilhelm von (1827): Werke in fünf Bänden, Bd.4. Hg.v. Flitner, Andreas / Giel, Klaus. Darmstadt: Wissenschaftliche Buchgesellschaft 1981.

Klein, Julie Thompson (2012): A Taxonomy of Interdisciplinarity. In: Frodeman, Robert (ed.): The Oxford Handbook of Interdisciplinarity. Oxford: Oxford University Press, S. 15 f.

Nietzsche, Friedrich (1873): Über Wahrheit und Lüge im außermoralischen Sinne. In: Colli, Giorgio/Montinari, Mazzino (Hg.): Kritische Studienausgabe, Bd. 1.2. Durchges. Aufl. München: Deutscher Taschenbuch Verlag, S. $873-890$.

Schnieder, Lars / Stein, Christian/Schielke, Arno (2011): Terminologiemanagementsysteme der nächstens Generation - Schlüssel für den Fachwortschatz. In: eDITionFachzeitschrift für Terminologie, Nr. 1, S. 26-31.

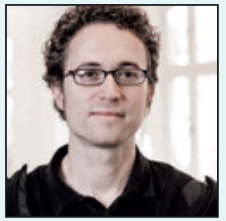

christian.stein@hu-berlin.de

Wissenschaftlicher Mitarbeiter

Basisprojekt: Architekturen des Wissens

Disziplin: Germanistik, Informatik

Christian Stein forscht im Interdisziplinären Labor zu den Funktionsweisen von Interdisziplinarität selbst. Dazu analysiert er verschiedenste Messdaten zu Arbeitsverhalten, Raumnutzung und Kommunikation, die in einer gesonderten Experimentalzone des Exzellenzclusters erhoben werden. Das Ziel ist es, Rahmenbedingungen und Teamzusammensetzungen für gelingende Interdisziplinarität herauszuarbeiten, um Projekte innovativer und erfolgsversprechender aufstellen zu können. Themenfelder seiner Forschungen als PostDoc sind zudem Datenschutz, Modelltheorie, Raumtheorie, Semantic Web, Semiotik und Terminologie sowie die AG Big Data der Jungen Akademie. 


\section{mauern}

\section{Wissensstruktur und Strukturwissen der Architektur}

Wissensstrukturen in ihrer Gesamtheit in einem Interdisziplinären Labor neu zu denken bedeutet zunächst, sich darüber verständigen zu müssen, wie einerseits Wissen und andererseits Struktur in den jeweiligen Disziplinen gedacht wird. Die Vielzahl der Disziplinen, die am Interdisziplinären Labor beteiligt sind, führt zu einer Vielzahl unterschiedlicher Konzepte von Wissen und Struktur, unterschiedlicher Semantiken zur Beschreibung dieser Konzepte und unterschiedlicher Methoden, mit Wissen und Strukturen zu operieren. In dieser disziplinären Verschiedenheit finden sich aber auch Überschneidungen und Ähnlichkeiten. Die Herausforderung besteht nun darin, aus diesem differenten, additiven, multidisziplinären Wissenswissen und Strukturwissen ein integratives, interdisziplinäres Wissenswissen und Strukturwissen zu entwickeln, welches die Grundlage für ein neues Denken von Wissensstrukturen bildet. Dabei kann es nicht darum gehen, den kleinstmöglichen Nenner zu finden, sondern das größtmögliche Potenzial sichtbar und operativ wirksam zu machen. Das wiederum geht nur, wenn die einzelnen Disziplinen explizit ihren fächerspezifischen Blick darlegen und kommunizieren. Jede der disziplinspezifischen Perspektiven fordert dazu auf, gemeinsam in jeweils unterschiedlichen Arten und Ausmaßen über Wissensstrukturen nachzudenken.

Was kann nun die Architektur dazu beitragen? Worin liegt das Potenzial einer Perspektivierung, die von der Architektur aus in ein interdisziplinäres Denken von Wissensstrukturen eingebracht werden kann? 
Im Folgenden soll die These entwickelt werden, dass der disziplinäre Blick der Architektur auf ihr Strukturwissen entscheidende Impulse bereitstellt, ein interdisziplinäres Strukturwissen neu zu denken. Eine solche architekturspezifische Perspektivierung hätte dann im Weiteren einen entsprechenden Einfluss darauf, Wissensstrukturen in ihrer Gesamtheit neu zu denken.

\section{Die architektonische Basis des Strukturbegriffs}

Das Strukturwissen, wie es in den Natur- und Geisteswissenschaften heute verwendet wird, ist ein vergleichsweise junges Wissen, das erst mit Beginn des 20. Jahrhunderts die wissenschaftlichen Disziplinen grundlegend geprägt hat. Der Strukturbegriff an sich ist jedoch viel älter und hat seinen Ursprung auch nicht in einem wissenschaftlichen, sondern in einem gestalterischen Kontext - dem der Architektur. Etymologisch lässt sich der Strukturbegriff auf das lateinische Wort structura zurückführen, welches die Bezeichnung für den Mauerwerksverband ist. So kann man in dem ältesten überlieferten, umfassenden Architekturtraktat De architectura libri decem von Vitruv um 30 v. Chr. nachlesen:

»Structurarum genera sunt haec: reticulatum, quo nunc omnes utuntur, et antiquum, quod incertum dicitur.«

»Die Arten des Mauerwerks sind folgende: reticulatum (netzförmiges Mauerwerk), das jetzt alle verwenden, und ein altertümliches, das opus incertum (unregelmäßiges Bruchsteinmauerwerk) genannt wird.«'

Mit dem lateinischen Wort struere wird entsprechend die dazu notwendige Tätigkeit des Mauerns und mit structor der römische Maurer bezeichnet. Der Begriff Struktur geht also aus der architektonischen Praxis des Mauerns hervor (Abb. 1-3).

Das ist alles nicht neu, aber verwendet wurde dieser etymologische Verweis auf die architektonische Basis des Strukturbegriffs meist nur als kleines, folgenloses Aperçu - ein konkreter wissenstheoretischer Blick auf die Tätigkeit des Mauerns wurde jedoch nicht daran angeschlossen. Zu traditionell, zu innovationsarm, zu banal erschienen wohl diese Tätigkeit und deren Ergebnisse. Damit wurde jedoch die Möglichkeit außer Acht gelassen, ein vormodernes Strukturwissen herauszuarbeiten, welches ausschließlich gestalterisch und noch nicht von einem wissenschaftlich formatierten, modernen Strukturbegriff geprägt ist.

Zu diesem Argument tritt ein weiteres: Die digitale Revolution und Materialinnovationen haben die heutige Architektur in Entwurf und Produktion grundlegend verändert, und mit Computational-Design-Strategien und Robotertechnologie wird die klassische Praxis des Mauerns neu in den Blick genommen. So hat zum Beispiel das Architekturbüro Gramazio \& Kohler seit Anfang der 20ooer-Jahre begonnen, an der ETH Zürich zu den Möglichkeiten von robotergestützter

1 Zitiert aus der von Carl Fensterbusch übers. und komm. Ausg.: Fensterbusch 1996, 102-103. 


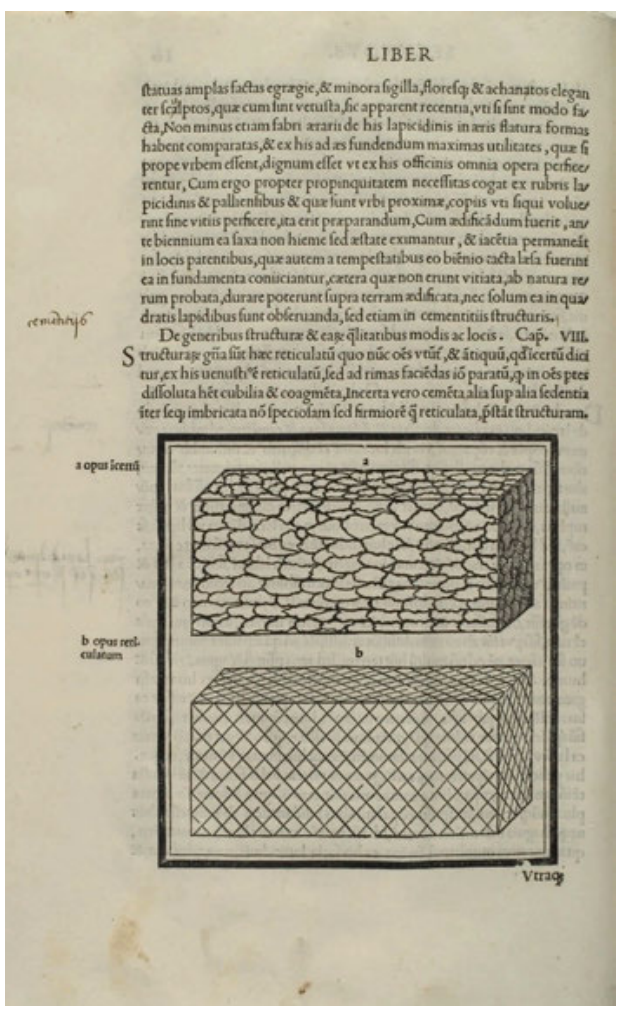

SECVNDVS.

IS

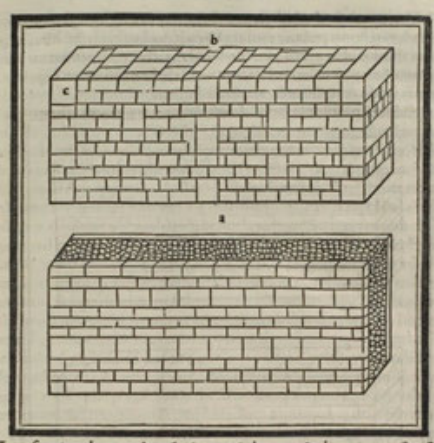

Inq fi quis uoluentex his cómétaris aliaduertere \& eligere genus ftructu rx,ppetuitatis poteritrónem hére, Non.n. gux funte molli centéto fubeili

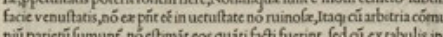

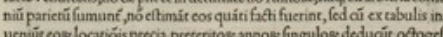

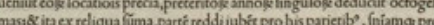

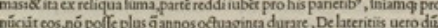

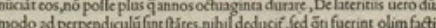

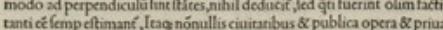

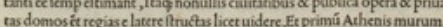
as domos et regiase latere ftruetts licet uidere, Ex primú Athenis murum,

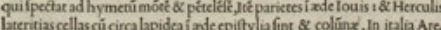

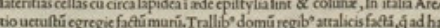

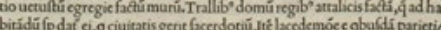

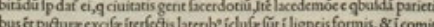

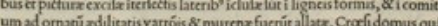
um ad ornañ xdilitatisvarrós \& murenx fucruit allacx, Croctid domus qu

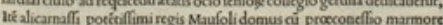

begrzagru c Dimomest

2. empleas.

\section{LIBER}

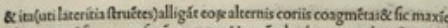
ad arernitaré himas perticiút virtures, Hax aúr duobus generibus ftruune,

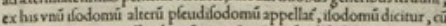
omniacoraxqua craffitudine fuerint itructe, pleudifodomum cim im pares $\&$ inxquales ordines coriorum diriguntur.

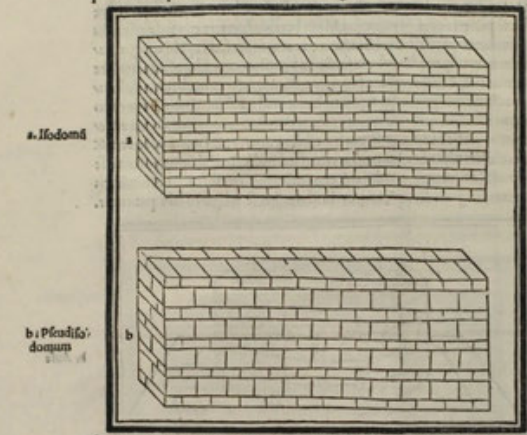

Eavtraq fír ideo firma, primú $q$ ipfaceméca fír fouffa \& folida pprietate

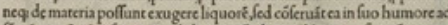
limá uetuftaté, pfaq copecubila primú plana \& librata pofita nó patiun tur nuere matenia, fed ppetua parieni craftitudine religata cótinḱr ad fum.

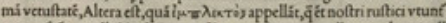
Quols frótes pollứt, sclio ita uti fut nat2,cumateria collocata alternis al.

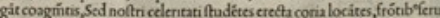
unt, Ki medio farcuút fratis fepati cú materia cemétis, ita tres fulcitant in

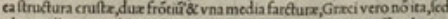
plana collocítes \& Lógitudines coriogs alternis cosemétisi craff mudiné in

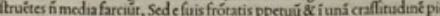

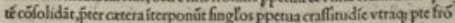

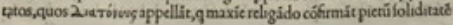

Abb. 1-3: Diese Seiten entstammen der Vitruv-Ausgabe von 1511, die von Giovanni Giocondo in Venedig herausgegeben wurde, und zeigen unterschiedliche Mauerwerksverbände - structurae. 
Fabrikation in der Architektur zu forschen und dabei die Praxis des Mauerns reaktiviert. Prominent angewendet wurde dieses robotergestützte Mauern unter anderem in dem Bau der Wände des Weinguts Gantenbein in Fläsch (Schweiz) 2006 (Abb. 4), der Installation Structural Oscillations auf der 11. Architektur Biennale Venedig 2008 (Abb. 5) und schließlich in dem Projekt Flight Assembled Architecture von 2011 - der wohl zur Zeit innovativsten Version eines Mauerns (Abb. 6, 7). ${ }^{2}$ In Zusammenarbeit mit dem System- und Roboterspezialisten Raffaello D'Andrea ließ das Architekturbüro das Architekturmodell einer Turmstadt bauen, in der die einzelnen Modell-Bauelemente mithilfe von Flugrobotern aufeinandergesetzt werden.

Ein genauerer Blick auf das Mauern scheint also mehr als angebracht und zieht folgende zwei Fragen nach sich: 1) Welches Wissen - welche Wissensbereiche, -formen und -formate lassen sich beim Mauern unterscheiden, charakterisieren und benennen? 2) Wie ist dieses Wissen strukturiert, wie lässt sich die Struktur des architektonischen Wissens beschreiben?

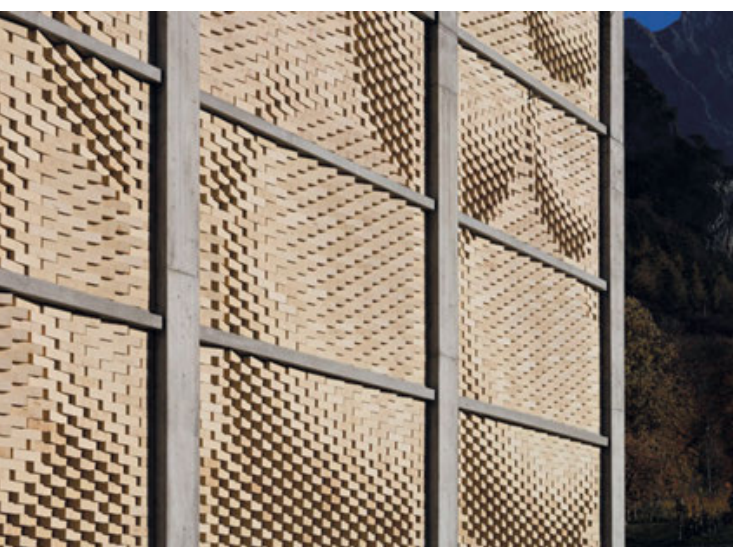

Abb. 4: Mit Robotertechnologie hergestellte Außenwand des Weinguts Gantenbein, 2006.

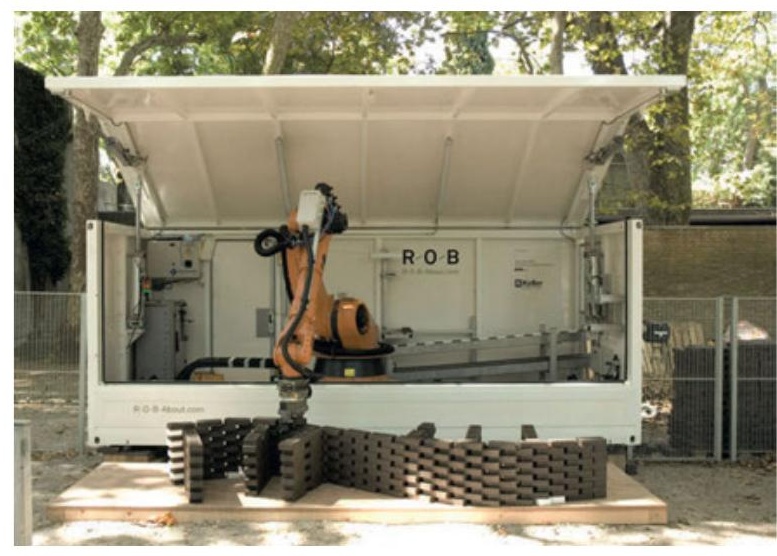

Abb. 5: Wandaufbau durch einen Roboter für die Installation Structural Oscillations in Venedig, 2008. 
Was dieses Modell interessant macht, ist, dass es nicht darauf abzielt, das Endprodukt - die Turmstadt - zu simulieren, sondern den Bauprozess. Die architektonische Praxis und Gestaltungstechnik, die diesem Prozessmodell unterliegt, ist die des Mauerns: Einzelne Elemente werden nach einem bestimmten System neben- und übereinander so angeordnet, dass sie eine raumdefinierende Mauer bilden. In Flight Assembled Architecture wird somit simuliert, wie die basale architektonische Praxis des Mauerns bei heutigem Wissensstand in einem städtebaulichen Maßstab durchgeführt werden könnte.

Die Frage ist nun, welches Wissen benötigt wird, um einen solchen Mauerprozess durchzuführen. Welche Entscheidungen müssen gefällt werden, welches Wissen wird dafür benötigt und woher kommt es? - Kurz: Welches Wissen steckt hinter den Entscheidungen des Architekturteams und wie ist es strukturiert?

\section{Wissensbereiche des architektonischen Strukturwissens}

Mit einem konkreten Blick auf die Installation werden im Folgenden vier Wissensbereiche unterschieden und benannt, welche die Grundlage für die einzelnen Entwurfs- und Realisierungsentscheidungen des Architekturteams bilden. Innerhalb dieser Wissensbereiche muss das Architekturteam Entscheidungen fällen mit dem Ziel, den beabsichtigten Zweck der architektonischen Installation zu realisieren. Diese vier Wissensbereiche benenne ich mit den Begriffen Material, Technik, Raum und Kultur.

1) Das Architekturteam muss sich für ein Material entscheiden, mit dem gemauert werden soll. Dieses Material muss vielen - widerstreitenden - Kriterien genügen: Es muss einerseits leicht formbar sein, denn es soll in eine immer gleiche Blockform gebracht werden und andererseits als Blockform formstabil sein. Außerdem muss es leicht sein, damit es von den Flugrobotern transportiert werden kann. Das Material sollte zudem kostengünstig sein, aber auch umweltverträglich. Es sollte schnell zu produzieren, aber auch nachhaltig sein. Diese Liste ließe sich beliebig weiterführen. Alle diese Entscheidungen benötigen als Grundlage ein umfassendes Materialwissen.

2) Das Architekturteam muss sich für eine Technik entscheiden, mit der gemauert werden soll. Dazu gehört nicht nur die Entscheidung, das Mauern mithilfe von Flugrobotern durchzuführen, sondern auch die Entscheidung, in welcher Reihenfolge und in welchem Winkel zueinander die einzelnen Blöcke aufeinandergestapelt werden, wie die einzelnen Elemente untereinander verbunden werden, wie die Blöcke durch die Flugroboter festgehalten und mit welcher Software sie gesteuert werden. Für diese Entscheidungen benötigt das Architekturteam ein Technikwissen.

3) Das Architekturteam muss sich für einen Raum entscheiden, in dem gemauert werden soll. Wie muss der Raum beschaffen sein, dass er die Bereitstellung der Mauerelemente, die Flugbewegungen der Flugroboter und das Produkt - die Mauer - ermöglicht? Das Architekturteam 
benötigt ein Wissen darüber, wie sich Material- und Technikentscheidungen im Medium des Raumes konkretisieren lassen und welche Auswirkungen sie wiederum auf den Raum haben, wie sie ihn gestalten. Dabei geht es nicht allein um physische Eingriffe und deren physische Auswirkungen, sondern auch um die damit verbundene veränderte Raumwahrnehmung. Dafür ist ein umfassendes Raumwissen notwendig.

4) Das Architekturteam muss sich für eine Kultur entscheiden, in der gemauert werden soll. Die von ihnen getroffenen Material-, Technik- und Raumentscheidungen müssen jenseits ihrer Zweckerfüllung Sinn machen. Die Technikentscheidung, mithilfe von Flugrobotern zu mauern, macht zum Beispiel in einem hochtechnologisch industrialisierten Umfeld Sinn, aber nicht in einem Kontext ohne diese technischen Möglichkeiten. Dieser kulturspezifische Blick richtet sich außerdem auf den Zweck selbst und hinterfragt ihn kritisch. Der Zweck - hier der Bau einer Turmstadt - macht nur in einem kulturellen Kontext Sinn, in dem die Bodenpreise hoch sind, eine starke Bevölkerungsdichte herrscht und genügend Menschen bereit sind, in einer solchen Turmstadt zu wohnen oder zu arbeiten. Die Entscheidungen benötigen ein breites Vergleichswissen, welches als die Funktion des modernen Kulturwissen zu verstehen ist. ${ }^{3}$

In der Unterscheidung der beiden ersten Wissensbereiche - dem Materialwissen und dem Technikwissen - scheint die Unterscheidung zwischen knowing-that und knowing-how von Gilbert Ryle auf. Der britische Philosoph hatte 1949 in seinem Buch The Concept of Mind diese beiden Wissenskategorien eingeführt, um zwischen dem klassischen Tatsachenwissen - dem knowing-that - und einem Technik- und Handlungswissen - dem knowing-how - zu unterscheiden. ${ }^{4}$ In einer Weiterführung dieser Unterscheidungs- und Bezeichnungslogik soll hier das mediale Raumwissen entsprechend als ein knowing-where und das vergleichende Kulturwissen als ein knowing-different bezeichnet werden:

\begin{tabular}{|c|c|c|}
\hline MATERIAL & MATERIALWISSEN & KNOWING THAT \\
\hline TECHNIK & TECHNIKWISSEN & KNOWING How \\
\hline RAUM & RAUMWISSEN & KNOWING WHERE \\
\hline KuLtUR & KULTURWISSEN & KNOWING DifFERENT \\
\hline
\end{tabular}

3 Siehe Baecker 2003.

4 Siehe Ryle 1949. 


\section{Wissensformen und -formate des architektonischen Strukturwissens}

Architektonisches Strukturieren lässt sich als ein komplexer Entscheidungsprozess beschreiben, der auf vier unterschiedliche Wissensbereiche zurückgreifen muss, damit er Sinn macht. Dabei sind Material, Technik, Raum und Kultur keine originär architektonischen Wissensbereiche in dem Sinne, dass ihr Wissen von der Architektur generiert wird. Vielmehr werden Material-, Technik-, Raum- und Kulturwissen von anderen Akteuren hervorgebracht: den Wissenschaften, dem Handwerk und der Kunst. Entsprechend findet sich eine Vielzahl von unterschiedlichen Wissensformen: wissenschaftliche Wissensformen der Natur- und Geisteswissenschaften, handwerklich-technische Wissensformen und künstlerische Wissensformen. Eine eindeutige Zuordnung einer Wissensform zu einem Wissensbereich gibt es dabei nicht. Vielmehr ist jeder Wissensbereich selbst inhomogen und beinhaltet unterschiedliche Wissensformen: Das Materialwissen wird zu einem beträchtlichen Teil von naturwissenschaftlichen Disziplinen wie der Physik, Chemie, aber auch der Biologie gespeist. ${ }^{5}$ Aber auch das handwerkliche Wissen über Materialien findet sich in diesem Wissensbereich. Ebenso lassen sich im Technikwissen sowohl wissenschaftliches Wissen aus den Ingenieur- und Planungswissenschaften und der Informatik sowie das Wissen aus der handwerklichen Praxis ausmachen. ${ }^{6}$ Ein Raumwissen findet sich vor allem bei den Geowissenschaften, der Raumsoziologie aber auch bei raumbezogenen künstlerischen Praxen. Das Kulturwissen wird wiederum hauptsächlich von geisteswissenschaftlichen Disziplinen wie der Kulturwissenschaft und den Geschichtswissenschaften, aber auch durch die Sozialwissenschaften gespeist.

Zu diesen unterschiedlichen Wissensformen treten zusätzlich zwei verschiedene Wissensformate: explizite und implizite Wissensformate. Diese stehen quer zu den oben genannten Wissensformen, das heißt, in jeder Wissensform findet sich sowohl explizites als auch implizites Wissen. Das Verhältnis differiert nach Wissensform, sodass zum Beispiel in den wissenschaftlichen Wissensformen eher explizites Wissen anzutreffen ist, während handwerkliches Materialwissen zu einem großen Teil aus einem impliziten Wissen besteht.

Fasst man die obigen Überlegungen zu den Wissensbereichen, -formen und -formaten zusammen, so kann das architektonische Strukturwissen als ein synthetisches Wissen aus unterschiedlichen Wissensbereichen (Material, Technik, Raum, Kultur), Wissensformen (wissenschaftlich, handwerklich, künstlerisch) und Wissensformaten (explizit, implizit) definiert werden. ${ }^{7}$

5 Die Fächer kulminieren zusammen in der interdisziplinären Materialwissenschaft.

6 Bezeichnenderweise firmieren Ingenieur- und Planungswissenschaften mit der Informatik in den letzten Jahren unter dem Label der Technikwissenschaften.

7 Architektonisches Strukturwissen lässt sich damit nicht nur als komplex, sondern geradezu als enzyklopädisch bezeichnen. Genau darin liegen auch seine besondere Eigenart und Unterschiedlichkeit zum Strukturwissen anderer Disziplinen. 


\section{Wissensstruktur des architektonischen Strukturwissens}

Nachdem nun vier Wissensbereiche, drei Wissensformen und zwei Wissensformate analytisch unterschieden, inhaltlich charakterisiert und terminologisch benannt worden sind, welche die Wissensgrundlage für die architektonische Praxis des Mauerns und damit die des architektonischen Strukturierens bilden, stellt sich die Frage nach der spezifischen Struktur dieses Strukturwissens. Die Herausforderung besteht im Umfang und der Heterogenität des Wissens, auf das zurückgegriffen werden muss. Eine erste Operationalisierung dieser großen Wissensbereiche für die Architektur geschieht durch die sogenannten Fachdisziplinen. Sie sind es, die die großen Wissensbereiche nach architekturrelevantem Wissen durchsuchen, es sammeln, weiterentwickeln und den Architektinnen und Architekten zur Verfügung stellen. Diese Fachdisziplinen - zum Beispiel die Bauphysik im Bereich des Materialwissens, die Baukonstruktion im Bereich des Technikwissens, die Stadtsoziologie im Bereich des Raumwissens und die Baugeschichte im Bereich des Kulturwissens - sind jedoch heute schon wieder so groß und komplex geworden, dass sie hier weder die Entscheidungen signifikant erleichtern würden, noch können sie das Problem der Heterogenität lösen. Wenn das Strukturwissen ein synthetisches Wissen ist, dann stellt sich die Frage nach der Wissensstruktur des architektonischen Strukturwissens als diejenige, wie diese Synthetisierung genau abläuft. Konkret ergibt sich die Frage, in welchem Verhältnis bezüglich 1) Wertigkeit, 2) Zeitlichkeit und 3) Präsenz die einzelnen Wissensbereiche zueinander stehen: 1) Gibt es wichtige und weniger wichtige Wissensbereiche? 2) Müssen die Wissensbereiche in einer besonderen Reihenfolge in den Entwurfs- oder Realisierungsprozess eingebracht werden? 3) Kann auf einen Wissensbereich verzichtet werden?

Dazu soll noch einmal der Blick auf die konkreten Entscheidungen des Architektenteams gelenkt und nach den zugrundeliegenden Auswahlkriterien gefragt werden. Wonach muss sich beispielsweise die Materialentscheidung richten? Der Hinweis auf den Zweck ist an dieser Stelle natürlich einleuchtend und durchaus zutreffend, aber nicht ausreichend. Denn in der Architektur gibt es meist mehrere Möglichkeiten, um einen Zweck zu ermöglichen. Nach welchen Kriterien wird beispielsweise zwischen zwei gleichwertig erscheinenden Materialien entschieden? Hier ist der Kontext entscheidend, der aus allen schon gefällten und noch zu fällenden möglichen Entscheidungen aus den anderen Wissensbereichen besteht. Da jede Entscheidung auch andere ausschließt, muss das Architekturteam ins Kalkül ziehen, was damit in Zukunft ausgeschlossen wird. Eine Materialwahl muss nicht allein vor dem Hintergrund anderer möglicher Materialien getroffen werden, sondern auch immer zugleich vor dem Hintergrund vergangener und zukünftiger Technik-, Raum- und Kulturentscheidungen. Jede Änderung erfordert ein Überprüfen aller mit dieser Umentscheidung zusammenhängenden Entscheidungen. Denn Material-, Technik-, Raum- und Kulturentscheidungen hängen alle miteinander zusammen, sind aber nicht beliebig koppelbar. Nicht alle Materialien lassen sich für eine bestimmte Technik verwenden. Die Technikentscheidung, mit Flugrobotern zu bauen, bedeutet deshalb eine Einschränkung in der Materialwahl, denn es darf nur so schwer sein, dass die Flugroboter das Material noch transportieren können. 
Dieser rekursive Verknüpfungsprozess von Entscheidungen aus unterschiedlichen Wissensbereichen kennzeichnet den Synthetisierungsprozess des architektonischen Strukturwissens. Es wird gebildet durch das Netz in seiner dynamischen und offenen Gesamtheit. Dadurch wird auch deutlich, dass die einzelnen Wissensbereiche keine Hierarchie ausbilden. Vielmehr lässt sich in Anlehnung an aktuelle Modellierungstechniken in der Architektur die spezifische Struktur des architektonischen Strukturwissens als parametrisch bezeichnen. Beim parametrischen Modellieren werden Systeme durch die sie generierenden Parameter beschrieben, die als Variablen gespeichert und denen dann Werte zugewiesen werden können. Eine geometrische Form - zum Beispiel ein Kubus - wird nicht mehr durch seine exakte Geometrie, sondern über dessen generierende Parameter (Höhe, Breite, Länge) und die damit verknüpften Zahlenvariablen beschrieben. Bei Änderung eines Parameters werden aufgrund der bei der Modellerstellung formulierten Bezüge die übrigen Maße vom Rechner angepasst und nachgeführt. Hierbei würden die vier Wissensbereiche Material, Technik, Raum und Kultur den vier Parametern eines architektonischen Strukturwissens entsprechen, und jede Entscheidung wäre als ein Wert zu betrachten, der vom Architekturteam diesem Parameter zugewiesen wird. ${ }^{8}$ Zusammen ergibt sich damit für das architektonische Strukturwissen eine parametrisch vernetzte Wissensstruktur aus zweckgerichteten, raumbasierenden und differenzsensiblen Entscheidungen über Materialien und Techniken.

Die Wissensmodellierung des architektonischen Strukturwissens, wie sie hier entwickelt worden ist, basiert einerseits auf einer begriffsgeschichtlichen Beobachtung (dass der Strukturbegriff auf das lateinische structura zurückgeht, welches die Bezeichnung für den Mauerwerksverband war) und andererseits der Beobachtung eines aktuellen Beispiel aus der Architekturpraxis (Flight Assembled Architecture). ${ }^{9}$ Ausgehend von diesen Beobachtungen werden im Folgenden drei architekturwissenschaftliche Forschungsfelder skizziert, die vom Prozess des Mauerns und dessen Interpretation als eines Strukturierungsprozesses aus jeweils einen besonderen Fokus auf 1) die Architektur, 2) das Material und 3) den Strukturbegriff legen.

8 Der Parameter Raumwissen stellt dabei sicher, dass die Material- und Technikentscheidungen im Medium des Raumes eine Strukturierung bewirken. Damit unterscheidet sich Architektur beispielsweise von zeitlichen Strukturierungen der Musik, die gleichwohl ebenfalls durch Material- und Technikentscheidungen konstruiert werden. Der Parameter Kulturwissen verhindert, dass sich eine stabile Konstellation festsetzt, denn es könnte ja immer auch anders sein, anders gedacht werden oder anders entworfen werden: Er hält damit das System Strukturwissen am Laufen und stellt sicher, dass es nicht stehen bleibt.

9 An dieser Stelle sei angemerkt, dass dieses Wissensmodell des architektonischen Strukturierens - welches anhand einer hochtechnologischen Variante des Mauerns gewonnen wurde - auch für handwerkliche Verfahren des Mauerns wie sie beispielsweise Vitruv über 2.000 Jahre früher beschrieben hat - zutrifft. 


\section{Die architekturwissenschaftliche Perspektive: mauern als Grundoperation der architektonischen Gestaltung}

Eine architekturwissenschaftliche Perspektive auf das Mauern wird eröffnet, indem nach dessen grundlegender Funktion gefragt wird. Welche Funktion hat das Mauern in der Architektur jenseits eines konkreten Zweckes? In einem abstrahierenden Sinne lässt sich das Mauern als eine Operation der Grenzziehung und der Abschirmung im Medium der Räumlichkeit verstehen. Dieses Abschirmen lässt sich als eine Operation des Schließens und Öffnens beschreiben, mit der ein Innen von einem Außen abgegrenzt wird, wobei Innen und Außen immer noch wechselseitig erreichbar bleiben. Damit wird die Operation des Schließens und Öffnens im Medium der Räumlichkeit zur grundlegenden architektonischen Operation. Die Abschirmung als Produkt dieser grenzziehenden Operation ist dann das basale Element der Architektur. ${ }^{10}$ Mit dem Prozess des Mauerns - also dem architektonischen Strukturieren - wird also nicht ein beliebiges, sondern das basale Element der Architektur generiert, welches dann selbst als Element dient, um Architektur zu formen - denn aus dem spezifischen Anordnen von Abschirmungen wird das architektonische Objekt konkretisiert. Es lässt sich hier also ein hierarchisches Stufenverhältnis erkennen: Wie Steine und Ziegel die Elemente der Mauer bilden, so bilden Mauern die Elemente der Architektur. Und so wie die Mauer über einen Strukturierungsprozess konkretisiert wird, so lässt sich die Konkretisierung der Architektur über einen analogen Strukturierungsprozess beschreiben. Denn für die Anordnung der einzelnen Mauern zu einem Bauwerk werden ebenso Material-, Technik-, Raum- und Kulturwissen benötigt. In der Technik des Mauerns lässt sich damit nicht nur das architektonische Strukturwissen beobachten, sondern vielmehr auch das architektonische Gestaltungswissen.

\section{Die materialwissenschaftliche Perspektive: mauern im Material}

Für eine materialwissenschaftliche Perspektivierung muss die Blickrichtung von der Mauer auf das Material der Mauerelemente selbst gerichtet werden - es muss in das Material gezoomt werden. Die aktuelle Biomaterial-Forschung mit ihren Möglichkeiten, bis in die Nanoebene von Materialien vorzudringen, hat gezeigt, dass diese nicht homogen aus einer einzigen Materialstruktur bestehen, sondern aus unterschiedlichen hierarchischen Strukturebenen. Je nach Größenmaßstab, je nachdem, wie tief in das Material gezoomt wird, zeigen sich unterschiedliche Strukturen. Diese hierarchischen Strukturebenen sind es, welche die Materialien so besonders potent machen und ermöglichen, dass sie unterschiedlichste Eigenschaften ausbilden. Dies wird in der Materialwissenschaft produktiv - gestaltend - genutzt: Für die Produktion von

10 Siehe dazu Dirk Baecker: »Sobald es um eine Abschirmung geht, die Innen und Außen trennt, geht es um Architektur, was auch immer diese Architektur bezwecken, welches Material auch immer verwendet sein und wie auch immer sie aussehen mag.« Baecker 1990, 90. 
Werkstoffen mit neuen Eigenschaften werden nicht mehr unterschiedliche Materialien kombiniert. Stattdessen werden die Strukturen in einem Material verändert. Die Materialwissenschaft beginnt auf Mikro- und Nanoebene Doppel-T-Träger, Röhren und Sandwichelemente zu produzieren, wie es im Makrobereich der Architektur getan wird. Der Begriff der »Architectured Materials «," der in die materialwissenschaftliche Diskussion eingeführt wurde, bringt sehr genau den Paradigmenwechsel zum Ausdruck. Damit wird das architektonische Strukturwissen zu einem notwendigen Wissen der Materialwissenschaft und die Architektur zu einem neuen Mitspieler im interdisziplinären Team der Materialwissenschaft.

\section{Die strukturwissenschaftliche Perspektive:}

\section{mauern als Ausgangspunkt einer transdisziplinären Strukturengeschichte}

Mit einem solchermaßen architektonisch charakterisierten Strukturbegriff als Ausgangspunkt könnte auf die weitere Geschichte des Strukturbegriffs zurückgeblickt und genauer gekennzeichnet werden, welche Transformationen wann stattfanden und welche disziplinären Aneignungen dafür verantwortlich sind. Grundlage hierfür wäre eine vertiefende historische Analyse des architektonischen Strukturierungsprozesses: Wie wurde er historisch beschrieben, wie lässt er sich heute beschreiben, wie wurde er damals und heute abgebildet? Daran anschließend ließen sich diese Beschreibungen und Darstellungen des architektonischen Strukturierens in ein Verhältnis setzten zu Beschreibungen und Abbildungen von Strukturierungsprozessen anderer Disziplinen - zum Beispiel in der Ethnologie, Linguistik, Soziologie, Physik und Biologie. Im Zusammenspiel eines geisteswissenschaftlich-hermeneutischen Strukturalismus und einer naturwissenschaftlich-analytischen Strukturforschung mit dem gestalterisch-synthetischen Strukturwissen der Architektur ließe sich so eine transdisziplinäre Strukturengeschichte entwerfen.

11 Bouaziz/Bréchet/Embury 2008. 


\section{Literatur}

Baecker, Dirk (1990): Die Dekonstruktion der Schachtel - Innen und Außen in der Architektur. In: Luhmann, Niklas / Bunsen, Frederick D. / Baecker, Dirk: Unbeobachtbare Welt. Über Kunst und Architektur. Bielefeld: Haux, S. $67-104$

Baecker, Dirk (2003): Wozu Kultur? 3.Aufl. Berlin: Kadmos.

Bouaziz, O. / Bréchet, Y. / Embury, J. D. (2008): Heterogeneous and Architectured Materials: A Possible Strategy for Design of Structural Materials. In: Advanced Engineering Materials, vol. 10, no. 1/2, pp. 24-36. Online: http:// onlinelibrary.wiley.com/doi/10.1002/adem.200700289/ epdf (last access: 5 May 2015).
Fensterbusch, Curt (Hg.) (1996): Vitruvii De architectura libri decem - Zehn Bücher über Architektur. 5. Aufl. Darmstadt: Primus.

Gramazio, Fabio/Kohler, Matthias / D'Andrea, Raffaello (2013): Flight Assembled Architecture. Orléans: Editions Hyx. Video der Installation online unter: http://vimeo. com/32962555 (zuletzt aufgerufen: 24. Mai 2015).

Ryle, Gilbert (1949): The Concept of Mind. London/ New York: Hutchinson's University Library.

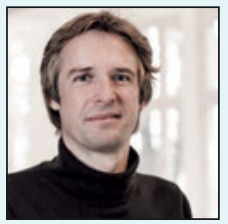

michael.duerfeld@hu-berlin.de

Wissenschaftlicher Mitarbeiter

Basisprojekt: Historische Strukturuntersuchungen im Labor

Disziplin: Architektur

Michael Dürfeld hat Architektur und Stadtplanung in Hamburg und Berlin studiert und am Fachbereich Architekturtheorie der Technischen Universität Berlin mit der Arbeit Das Ornamentale und die architektonische Form. Systemtheoretische Irritationen promoviert. Seit 2012 ist er wissenschaftlicher Mitarbeiter am Exzellenzcluster Bild Wissen Gestaltung. Seine Forschungsschwerpunkte liegen in der Interferenz von Architekturtheorie, Kunsttheorie und Systemtheorie. 


\section{Zu einer Unschärferelation der Modelle}

\section{Präzision und Produktivität mehrdeutiger Modelle in der Gestaltung}

Mehrdeutigkeiten, Unschärfen und Unbestimmtheiten von Modellen werden spätestens seit dem 16. Jahrhundert in Entwurfsprozessen gezielt produktiv gemacht. Vergleichsweise jung ist das Interesse an der theoretischen Durchdringung der dabei ablaufenden Vorgänge. Ihre Beschreibung ist mit charakteristischen Schwierigkeiten konfrontiert: Erstens erweist sich das Mehrdeutige, Unscharfe und Unbestimmte gegenüber dem konzeptuellen Zugriff als überaus fragil. Zweitens herrscht vielfach die Überzeugung, diese Form des Entwerfens sei eine Art natürlicher Prozess, dessen > Gesetze< von kulturellen Faktoren nicht beeinflusst seien. Drittens werden die Bemühungen um die theoretische Durchdringung des Entwurfs von einer Ex-post-Perspektive dominiert. Diese aber kann die Situationen nicht beschreiben, in denen die Entwerfenden sich unterwegs befanden - und daher auch nicht die Rolle der Modelle in diesen Situationen. Und viertens schließlich ist eine stabile Abgrenzung der Unbestimmtheit gegenüber der Bestimmtheit der Modelle in konkreten Entwurfsprozessen nicht zu beobachten. Stattdessen produzieren mehrdeutige, unscharfe und unbestimmte Modelle eine eigene Form der Präzision, während eindeutige, gute und zuverlässige Modelle umgekehrt eine spezifische Form der Mehrdeutigkeit, Unschärfe oder Unbestimmtheit produzieren. Diese beiden Phänomene werden im Folgenden exemplarisch dargestellt und nach ihren Zusammenhängen befragt. 


\section{Die Präzision der Unschärfe}

Der Kunsthistoriker Gottfried Boehm hat geschrieben, vor Bildern könne »der Mangel an Bestimmtheit in einen Überschuss an Sinn « umschlagen.' Leonardos Skizze für ein Gemälde von Anna, Maria und Kind von um 1506 bietet hierfür ein reiches Anschauungsmaterial (Abb. 1). ${ }^{2}$ Diese Studie wird hier als Beispiel gewählt, nicht obwohl, sondern weil sie bereits intensiv behandelt wurde. Als Reaktion auf einen Aufsatz des Kunsthistorikers Ernst Gombrich von 1952 wurde sie wiederholt auf ihren entwurfsmethodischen Gehalt hin untersucht. Im Zentrum dieser Untersuchungen stand, dass Leonardo mit Kohle und Tinte immer wieder dieselben Stellen auf dem Papier überzeichnete. Gombrich schreibt: »He works like a sculptor modelling in clay who never accepts any form as final but goes on creating, even at the risk of obscuring his original intentions. $\ll^{3}$ Gombrich verweist implizit auf Leonardos Prinzip, dass man keine gelungenen Kompositionen erschaffen könne, wenn man nicht bereit sei, die eigenen Entwürfe immer wieder zu verwerfen. Durch diese zunächst negative, auf die Verunklärung von Bildsinn gerichtete Strategie entsteht ein unerhörtes Potenzial, eine »explosive energy«, wie der Kunsthistoriker Martin Kemp schreibt. ${ }^{4}$ Sie besteht darin, dass in einer und derselben Skizze mehrere verschiedene Kompositionen gefunden werden können, auch solche, die Leonardo zuvor gar nicht in sie hineingezeichnet hatte. Dieser visuelle Sachverhalt kann nur bedingt mit Wolkenbildern oder verwitterten Mauern verglichen werden - auch dort lassen sich bekanntlich sinnhafte Formen finden. Bei der Studie für Anna und Maria mit dem Kind aber erzeugt Leonardo eine sozusagen optimierte Unbestimmtheit. Sie zielt bereits deutlich auf ein Gemälde ab und stellt damit einen gerichteten Möglichkeitsraum dar. Die Spielräume sind gleichsam maßgeschneidert, sowohl in Bezug auf das Entwurfsziel als auch auf die erforderlichen Freiheiten für das suchende Auge. In der Malerei des frühen 16. Jahrhunderts war die Loslösung von den antiken Vorbildern in vollem Gange. Zugleich wurden die Regeln der Mimesis immer weiter gedehnt und übertreten. In diesem Kontext kann man Leonardos Skizze eine methodologische Bedeutung attestieren: Sie folgt nicht den Regeln und dem Setting eines mimetischen Nachvollzugs, sondern etabliert einen neuartigen Werkprozess. Das Sujet ist dabei nicht so genau wie möglich abzubilden, sondern entsteht erst während des Entwerfens. Die historische Bedeutung der Zeichnung liegt darin, dass sie die Anfänge einer Entwurfsmethode lokalisierbar macht, die bis heute das Entwerfen und die Entwurfstheorien beherrscht. ${ }^{5}$ Dass Leonardos Methode die Zeiten überdauert hat, lässt sich mit einer Bemerkung des Architekten Günter Behnisch aus dem Jahre 2004 exemplifizieren. Er schreibt:

\footnotetext{
1 Boehm 2008, 204.

2 British Museum, London, Inv.-Nr. 1875-6-12-17.

3 Gombrich 1996, 211.

4 Kemp 1996, 16.

5 Siehe hierzu auch den Beitrag von Anouk Hoffmeister im vorliegenden Band.
} 
»Etwas wehmütig denken wir an unsere alten 6B-Skizzen, scheinbare Schmuddel-Skizzen, die unscharf waren, in denen man, wenn sie durch fortwährendes Überzeichnen zu kleinen Papierhügeln angewachsen waren, plötzlich Dinge durchscheinen sah, durch mehrere Lagen transparenten Papiers; Dinge, die man nicht kannte bis dahin; Dinge, die man nicht gezeichnet hatte, die einfach so entstanden sind, scheinbar aus sich selbst heraus. $\ll^{6}$

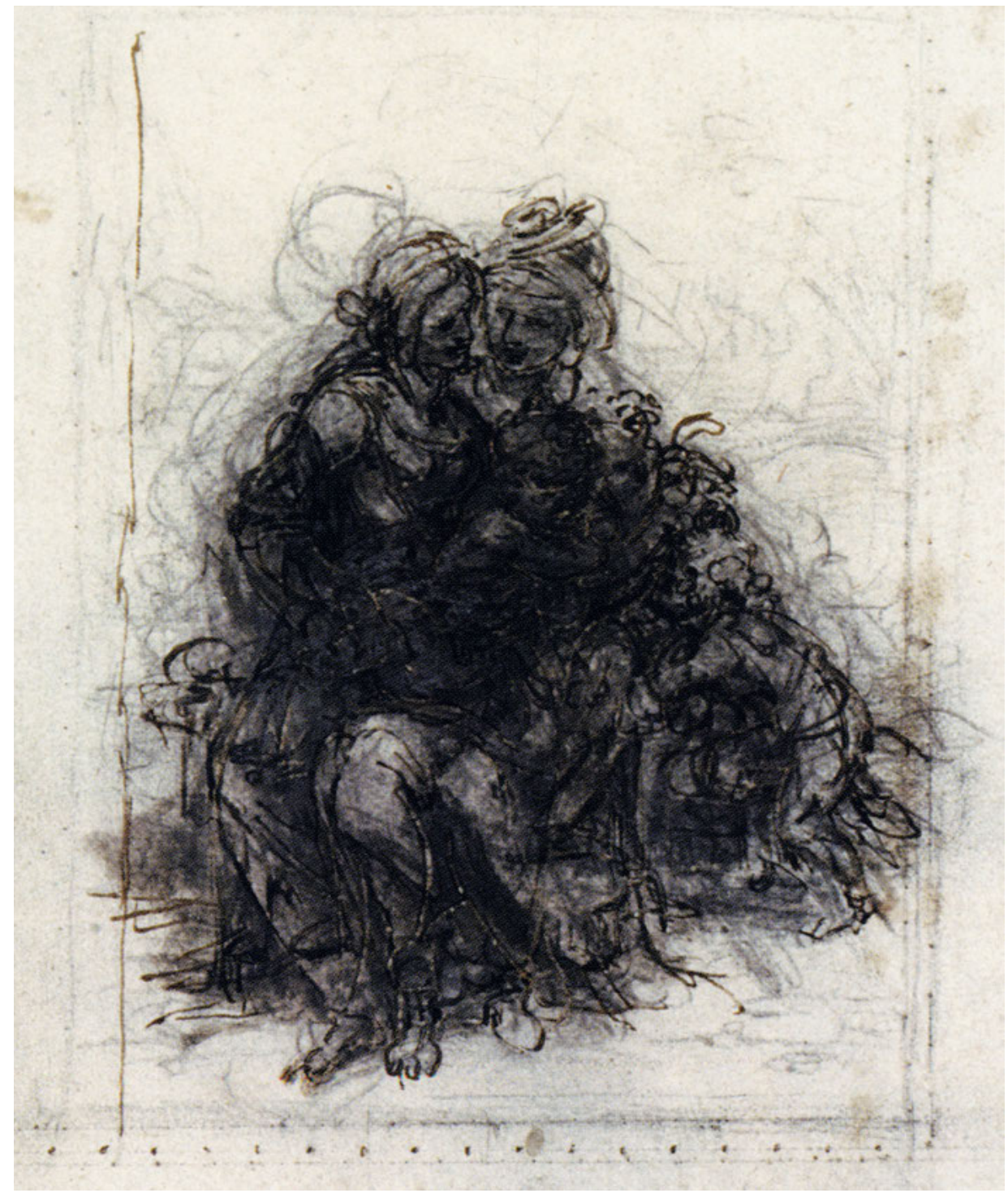

Abb. 1: Leonardo da Vinci, Maria und Anna mit Jesus und Johannes dem Täufer, Detail eines Studienblattes, 1506, Kohle, Feder, Aquarell, 26 x 19,5 cm, British Museum, London, Inv.-Nr. 1875-6-12-17.

6 Behnisch 2003, 34 . 
Die nebenstehende Abbildung zeigt einen Versuch, den beschriebenen Effekt zu instanziieren (Abb. 2). Behnisch liefert hier eine paradigmatische Beschreibung der Nutzbarmachung von Unschärfe, Unbestimmtheit und Mehrdeutigkeit in einem Gestaltungsprozess. Die visuelle Erscheinung der Überlagerung verschiedener Skizzen ist verwandt mit Moiré-Effekten und zugleich mit Rorschach-Klecksen. Sie erzeugt Muster und Verdichtungen, in deren Angesicht man sich neue Ideen zu entlocken vermag. Bei Behnisch schlägt ebenso wie bei Leonardo ein »Mangel an Bestimmtheit in einen Überschuss an Sinn « um. ${ }^{7}$ Leonardos Skizze ist dabei einer der historischen Vorläufer von Behnischs Vorgehen und vergleichbaren Entwurfspraktiken.

Die Produktivität von Leonardos Skizze lässt sich unter anderem daran bemessen, dass jede Darstellung andere Lesarten eröffnet. So konkretisieren sich auf dem Bildschirm eines Rechners andere Sichtungen als in der Projektion eines Videobeamers, den verschiedenen Drucken der verschiedenen Ausgaben von Leonardos Zeichnungen und freilich der Originalzeichung im British Museum. Jedes Material, jedes Medium und jeder Grad der Vergrößerung mischt seine Eigenheiten unter und treibt andere Imaginationen aus dem Liniengeflecht hervor. Auch Veränderungen des Blickwinkels und der Sichtweise wirken sich auf den Verlauf des Dialogs mit dem Bild aus. Es reagiert, mit dem Kunsthistoriker Michael Baxandall gesprochen, »sehr empfindlich [...] auf die verschiedenen Arten der Interpretationskunst - Muster, Kategorien, Schlussfolgerungen und Analogien -[,] die man an es heranträgt. $\ll^{8}$ Baxandall spricht hier von den multiplen Bedeutungen von Gemälden der Renaissance, aber seine Diagnose charakterisiert auch die visuelle Erscheinung von Leonardos Zeichnung.

Die einzelnen Imaginationen oder Sichtungen weisen eine eigentümliche Präzision auf. Diese lässt sich etwa an der besonders undeutlichen Region knapp rechts der Mitte der Skizze beobachten (Abb. 1). Hier kann das Auge die linke Hand der Maria und den Oberkörper des Christuskindes in verschiedenen Anordnungen imaginieren. Viele dieser Hybriden aus Projektion und Wahrnehmung sind präziser, als es allein die Farbspuren auf dem Papier hergeben. Der formsuchende Sehsinn überprägt dabei den grafischen Sachverhalt, indem er aus der Flut der visuellen Daten jeweils solche herausgreift und amalgamiert, die ein präzises Bild zu formen erlauben. Entscheidend für die frappante Präzision dieser Sichtungen ist hier, dass ihre Fragilität sehr genau der Feinheit der imaginierten Berührung der Hand entspricht. Die instabile visuelle Performanz dieser Region des Bildes vermittelt nachgerade synästhetisch das haptische Ereignis einer betont zärtlichen Berührung. Auch an anderen Stellen der Zeichnung blitzen aus dem Unbestimmten unerwartet präzise Figuren und Formen hervor. Inmitten des Unscharfen, Unbestimmten, Mehrdeutigen nistet also diese eigentümliche Form der Präzision.

7 Boehm 2008, 204.

8 Baxandall 1999, 46. 
<smiles>CCC(C)(C)C</smiles> 


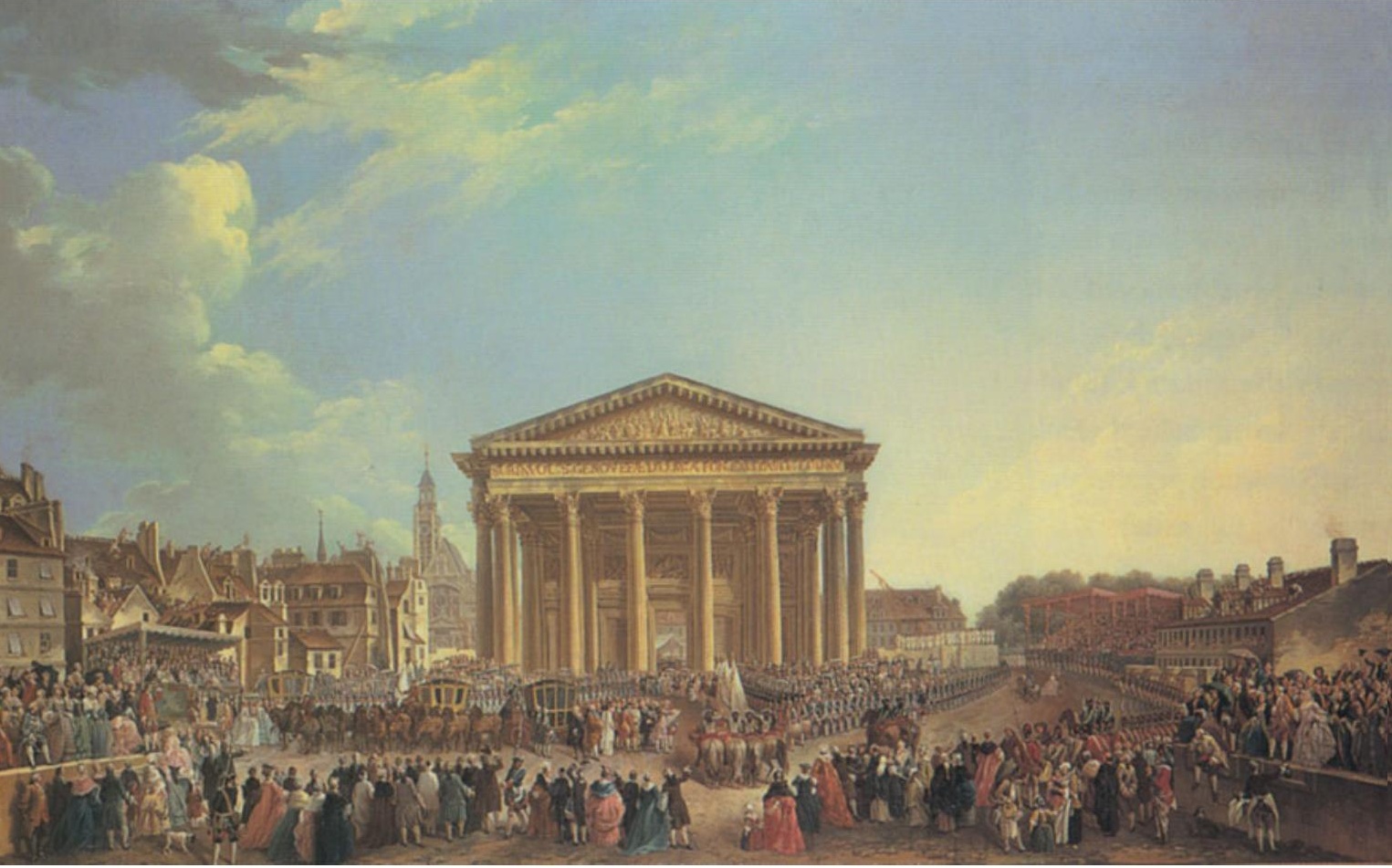

Abb. 3: Pierre-Antoine Demachy, Cérémonie de la pose de la première pierre de la nouvelle église Sainte-Geneviève, le 6 septembre 1765, Öl auf Leinwand, $81 \times 129 \mathrm{~cm}$, Musée Carnevalet - Histoire de Paris, Paris.

\section{Die Unschärfe der Präzision}

Ein scheinbar umgekehrtes Verhältnis lässt sich ebenfalls beobachten. So stellt sich bei exakten oder getreuen Modellen eine eigene Form der Unschärfe, Unbestimmtheit und Mehrdeutigkeit ein. Dies lässt sich an einem Gemälde beobachten, das 1765 von Pierre-Antoine Demachy geschaffen wurde (Abb. 3). Angeblich zeigt es die Grundsteinlegung für die Kirche von Sainte-Geneviève in Paris, also das Panthéon. Zwischen dem Bild und dem angeblichen Sujet herrscht ein offenkundiger Widerspruch: Warum steht bei der Grundsteinlegung bereits die gesamte Front des Gebäudes? Die Antwort lautet: Hier stand kein aufgemauertes Gebäude, sondern ein Eins-zu-eins-Modell. Bemalte Leinwände wurden für die Festlichkeiten an einem Gerüst aufgehängt, um die körperliche Wirkung des zu errichtenden Gebäudes erfahrbar zu machen. Auf diesen Umstand weist das Bild aber nicht wirklich hin. Es zeigt keine bemalten Leinwände, sondern ein Gebäude, also nicht das Modell, sondern das, worauf es verweist.

Das Bild vollendet, was das Modell begonnen hat: das Aufgehen in seinem Bezugsgegenstand. Auf diese Weise macht das Gemälde die allgemeinere Tendenz der Modelle zur Verunklärung konstitutiver Grenzen exemplarisch dingfest. Bei diesem in den Künsten und Wissenschaften vertrauten und gefürchteten Vorgang gilt die folgende Regel: Für je exakter und zuverlässiger 
das Modell gehalten wird, desto stärker verschwimmt es mit seinem Bezugsgegenstand. Dieser Vorgang generiert eine eigene Form der Unbestimmtheit und Mehrdeutigkeit, da nicht mehr eindeutig bestimmt ist, wovon die Rede ist. Die Unschärfen sind hier sogar noch größer als bei Leonardos Studie oder bei Behnischs 6B-Skizzen. Denn nun verschwimmen nicht lediglich Linien vor Augen, sondern weitaus größere Zusammenhänge wie Anfang und Ende, Idee und Umsetzung, hier und dort, jetzt und einst. Fundamentale Orientierungspunkte also geraten in Bewegung, und der gesamte Einzugsbereich des Modells verwandelt sich in eine dynamische Epistemologie. Man könnte dem präzisen Modell daher, mit Kemp gesprochen, eine weitaus größere explosive Energie attestieren als der Zeichnung Leonardos. Im Exakten, Getreuen, Isomorphen nistet also diese eigentümliche Form des Mehrdeutigen, Unscharfen, Un- oder Unterbestimmten.

Demachys Gemälde kann als ikonischer Kommentar zu den zahlreichen Warnungen angeschaut werden, das Modell nicht mit dem zu verwechseln, auf das es verweist. Historisch gesehen sind diese Warnungen zuerst in der Renaissance im Bereich der Architektur und der Skulptur nachweisbar und greifen nachher auf die Wissenschaften, die Wissenschaftstheorie und die Pädagogik über. Der Wissenschaftshistoriker Georges Canguilhem hat auf dieses Problem im Zusammenhang mit frühen Computermodellen in der Biochemie hingewiesen. Die untenstehende Abbildung zeigt ein 1966 entwickeltes Rendering eines Modells des Moleküls Zytochrom c, eines Zellfarbstoffes und Elektronentransporters (Abb.4).

Abb. 4: Rendering eines Modells vom Zytochrom c.

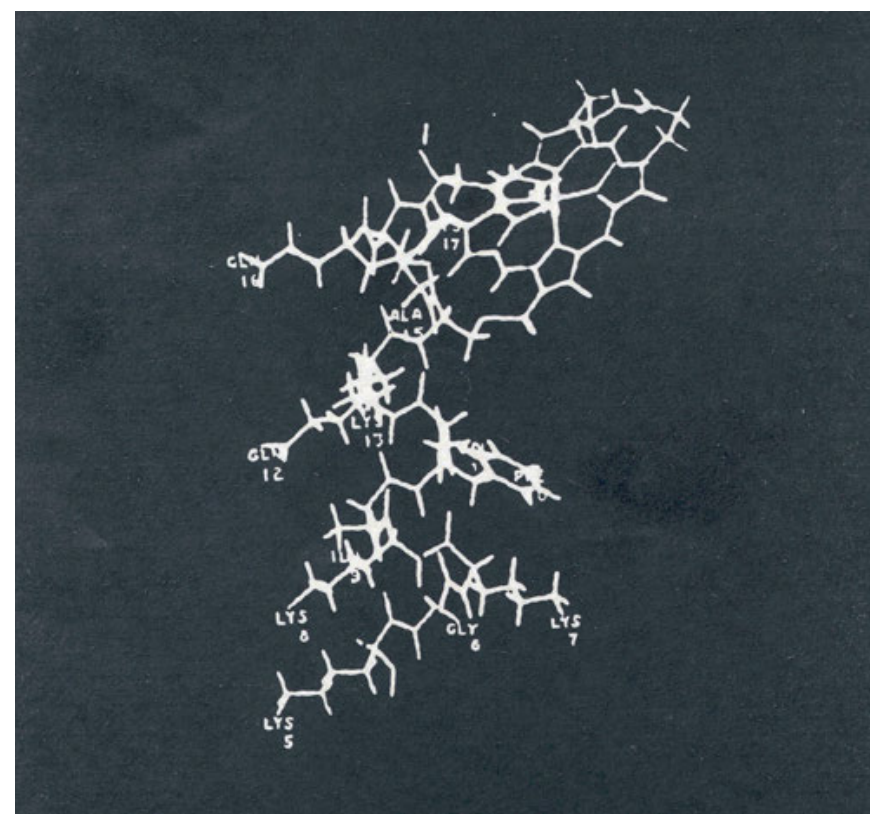


Das Bild ist später entstanden als Canguilhems Aufsatz, dürfte aber denjenigen ähneln, die er vor dem geistigen Auge hatte. Er schreibt: »A bad model, in the history of science, is that which the imagination evaluates as a good one. ${ }^{9}$ Das für gut gehaltene Modell ist schlecht und dies, gerade weil es für gut gehalten wird. Canguilhem fragt daher: »Could not one say [...], that in biology the models which have the chance of being the best are those which halt our latent tendency to identify the organic with its model? $\ll^{10}$ Die besten Modelle nach Canguilhem verhindern genau das, was in Demachys Bild studierbar ist: die Verwechslung des Modells mit seinem Bezugsgegenstand. Dabei liegt die größte Präzision in Demachys Gemälde gerade im Fehlen von Hinweisen auf die bemalten Leinwände und in der weitgehenden Unsichtbarkeit des eigentlichen Sujets. Die Verwechselbarkeit bietet ein Anschauungsmodell dafür, dass auch wissenschaftliche Modelle vielfach der latenten Tendenz keinen Einhalt gebieten, Modell und Bezugsgegenstand miteinander zu identifizieren.

Man könnte diese Tendenz in Anlehnung an eine Beobachtung Horst Bredekamps das Brillanzproblem der Modelle nennen." Es besteht in einer Unschärfe oder Mehrdeutigkeit, die als solche nicht kritisch distanziert respektive methodisch eingeholt ist. Das Fehlen geeigneter Gegenmaßnahmen geht in erster Linie darauf zurück, dass das partielle Aufgehen des Modells in seinen Bezugsgegenständen kein Unfall ist, sondern Teil der Modellmethode. Die sozusagen mutwillige Verunklärung der Unterschiede ist in mehrerlei Hinsicht produktiv: Sie fungiert als eine Art Klebstoff, mit dem man Ungleiches miteinander verbinden und auf diese Weise Ordnungen schaffen sowie Hypothesen formulieren kann. Sie löst die Gestaltenden und Forschenden aus den Zwängen der Abläufe, Größenordnungen, Orte und Zeitpunkte und damit unter anderem aus den Zwängen anderer Modelle. Sie macht das Ungreifbare greifbar, leistet bei der Akquise von Geld und Unterstützung entscheidende Überzeugungsarbeit und ermutigt die Beteiligten zum Handeln. Der Preis für diese Qualitäten liegt in der Gefahr, das Angefangene, Canguilhems »latent tendency «, ${ }^{12}$ unbeabsichtigt zu vollenden und das Modell mit seinem Bezugsgegenstand zu identifizieren. Das für gut oder zuverlässig gehaltene Modell kann die Gestaltenden und Forschenden sich bereits am Ziel angelangt wähnen lassen und auf diese Weise das Denken und Handeln bis zur völligen Untätigkeit einschränken. Es kann entscheidende Fragen unterdrücken, den Status quo petrifizieren und damit ganze Disziplinen in die Bedeutungslosigkeit führen. Der Wissenschaftstheoretiker Richard Braithwaite hatte daher 1952 notiert: »The price of the employment of models is eternal vigilance. $\ll^{13}$ Diese ewige Wachsamkeit wird umso stärker geschwächt, je besser oder zuverlässiger das Modell zu sein scheint. Deshalb geht die Steigerung der Exaktheit oder Zuverlässigkeit eines Modells in einer Hinsicht mit einer Steigerung seiner Unbestimmtheit in einer anderen einher.

9 Canguilhem 1963, 517.

10 Ebd.

11 Vgl. Bredekamp 2010, 290.

12 Canguilhem 1963, 517.

13 Braithwaite 1953, 93. 


\section{Unschärferelation der Modelle}

Man könnte die hier dargestellten Wechselwirkungen als eine Art kulturtechnische Unschärferelation verstehen. Sie unterscheidet sich in nahezu jeder Hinsicht von dem durch Werner Heisenberg formulierten Prinzip, kann von diesem aber doch ein Verständnis dafür gewinnen, dass die Präzision eines Modells nicht beliebig steigerbar ist. An den Grenzen der Präzision muss sich der Blick vom Verhältnis zwischen Modell und Bezugsgegenstand lösen und auf die weitere Modellsituation ausdehnen. An die Stelle von Entsprechungsbeziehungen treten dann gänzlich kontraintuitive Verwechslungen und Verwandlungen, durch die unter anderem der Wille zur Präzision zu einem Agenten der Unschärfe wird. Die Bewusstmachung solcher Vorgänge kann für den Ausgang einer Modellierung entscheidend sein.

Insbesondere weisen die beiden hier dargestellten Formen von Unbestimmtheit Gemeinsamkeiten und Unterschiede auf. Ein Unterschied besteht darin, dass die Unbestimmtheit von Leonardos Skizze sichtbar ist, während sie bei dem für zuverlässig gehaltenen Modell verborgen bleibt. Der partielle Kontrollverlust kann in der Skizze produktiv werden, weil er unübersehbar ist und daher ins Kalkül gezogen wird. Bei dem scheinbar guten Modell kann der Kontrollverlust als ein Hemmnis auftreten, weil er sich dem Blick entzieht und daher unbemerkt sein Unwesen treibt. Bringt man ihn durch kritische Distanzierung wieder ins Spiel, so kann auch die Unbestimmtheit der zuverlässig gehaltenen Modelle produktiv gemacht werden.

Darüber hinaus gilt es, sich bewusst zu machen, dass das Modell selbst zur Hälfte an solchen und anderen Wirkungen beteiligt ist. Seine materielle, räumliche und sinnliche Präsenz zieht Gedanken auf sich und gibt sie, um seine aktuellen wie vermeintlichen Charakteristika bereichert, wieder zurück. Dies gilt auch für sogenannte theoretische und abstrakte Modelle, denen performative Effekte vielfach abgesprochen werden. Auch sie überprägen das durch sie Gedachte mit Aspekten ihrer aktuellen und vermeintlichen Verfasstheit. Darüber hinaus begünstigen oder vereiteln sie auch ihre Auffassung als exakte oder unscharfe Modelle. So können sie etwa durch ihre konkrete Machart der Verwechslung mit ihren Bezugsgegenständen teilweise entgegenwirken, etwa indem sie ihre Vorläufigkeit erkennbar ausweisen. Die meisten Modelle in den Wissenschaften werden hingegen mit Kennzeichen ihrer Exaktheit und Zuverlässigkeit ausgestattet, etwa um ihnen die Autorität einer gesicherten Erkenntnis oder das Auftreten eines Nachweises gewissenhafter Arbeit zu verleihen. Aber gerade in diesen Kennzeichen nisten, wie hier ausgeführt wurde, Unschärfen besonderer Art, die für das wissenschaftliche Weltbild eine nicht zu unterschätzende Rolle spielen. Letztlich sind bestimmte Fähigkeiten für eine erfolgreiche Modellanwendung ausschlaggebend, unter anderem: Das scheinbar klare und exakte Modell so anschauen zu können wie eine Zufallsskizze von Leonardo und in vorläufigen Konkretisierungen Interpretationen finden zu können, die so präzise sind wie eine physikalische Messung. ${ }^{14}$

14 Dieser Aufsatz ist Bernd Mahr gewidmet. Seine Einladung zum Nachdenken über Modelle möge durch das hier Gesagte bekräftigt werden. Die meisterhaft verfügten Schärfen und Unschärfen von Mahrs Modell des Modellseins weisen einen der vielen Wege, ihr zu folgen. 


\section{Literatur}

Baxandall, Michael (1999): Die Wirklichkeit der Bilder. Malerei und Erfahrung im Italien der Renaissance. Berlin: Wagenbach.

Behnisch, Günter (2003): Lebendige Gefühle. In: Lorenz, Peter (Hg.): Entwerfen. 25 Architekten - 25 Standpunkte. München: Deutsche Verlagsanst., S. 34-35.

Boehm, Gottfried (2008): Wie Bilder Sinn erzeugen. Die Macht des Zeigens. Berlin: Berlin University Press.

Braithwaite, Richard Bevan (1953): Scientific Explanation. A Study of the Function of Theory, Probability and Law in Science. Cambridge: Cambridge University Press.

Bredekamp, Horst (2010): Theorie des Bildakts. Frankfurt a. M.: Suhrkamp.

Canguilhem, Georges (1963): The Role of Analogies and Models in Biological Discovery. In: Crombie, Alistair (ed.): Scientific Change. Historical Studies in the Intellectual, Social and Technical Conditions for Scientific Discovery and Technical Invention, from Antiquity to the Present. New York: Basic Books, pp. 507-520.
Gombrich, Ernst (1996): Leonard's Method for Working out Compositions. In: Woodfield, Richard (ed.): The Essential Gombrich. Selected Writings on Art and Culture. London: Phaidon, pp. 211-221.

Kemp, Martin (1996): Leonardo da Vinci. Experience, Experiment and Design. London: V\&A Publications.

Levinthal, Cyrus (1966): Molecular Model-Building by Computer. In: Scientific American, vol.214, no. 6, pp. $42-52$. 


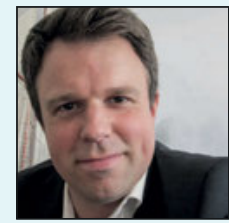

reinhard.wendler@khi.fi.it

Associated Member

Basisprojekt: Modelle in der Gestaltung

Disziplin: Kunst- und Bildgeschichte

Reinhard Wendler ist am Kunsthistorischen Institut Florenz als PostDoc des Forschungsverbunds Bilderfahrzeuge vom Warburg Institute in London beschäftigt und war PostDoc am Projekt Modelle als Akteure im Fachgebiet Formale Modelle, Logik und Programmierung sowie am Innovationszentrum Wissensforschung der Technischen Universität Berlin. Seinen Forschungsschwerpunkten geht er darüber hinaus in diversen Projekten am Exzellenzcluster Bild Wissen Gestaltung nach. Er studierte Kunstgeschichte, Musikwissenschaft und Philosophie an der Technischen Universität Berlin und der Humboldt-Universität zu Berlin. 



\section{Gestaltung als Synthese}




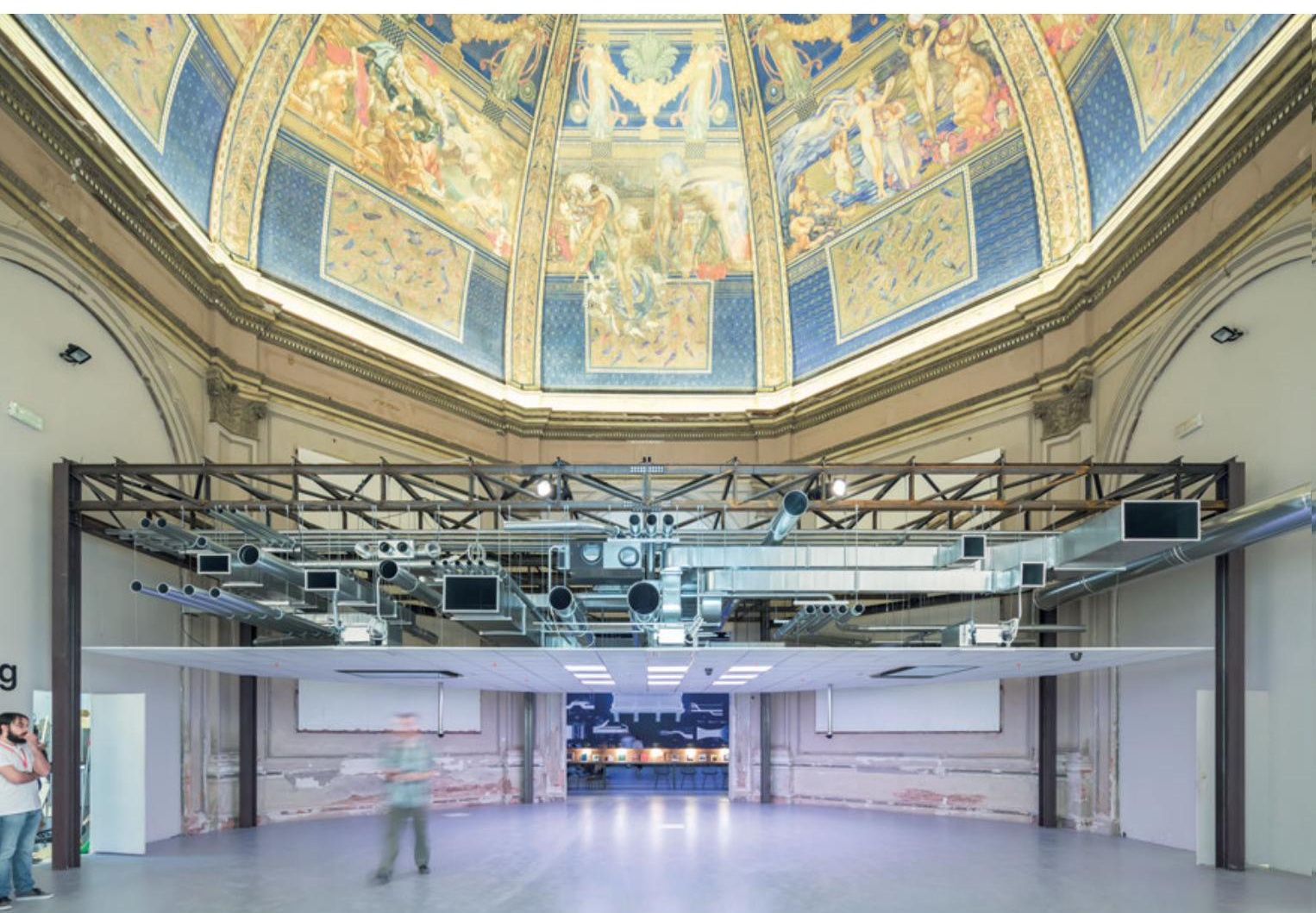

Abb. 1: Eingangshalle des Zentralpavillons, 14. Architekturbiennale Venedig, 2014. 


\section{Philipp Oswalt}

\section{Wissen - Nichtwissen - Entwerfen}

Der Architekt Rem Koolhaas montiert als Entrée zu der von ihm kuratierten 14. Architekturbiennale in Venedig 2014 unter die 1909 in klassischer Manier von Galileo Chini ausgemalte und vor Kurzem restaurierte Kuppel des Zentralpavillons eine moderne Klimadecke (Abb. 1). Mit dieser polemischen Raumcollage inszeniert er einen Konflikt und verweist damit auf die als krisenhaft empfundene Wirkung einer entfesselten und als kulturlos verstandenen Expansion der technisch-wissenschaftlichen Kultur. Zugleich schafft er eine dramatisierende Gegenüberstellung zweier Wissenskulturen, welche sich mit der Ausdifferenzierung der Architektur seit dem 17. Jahrhundert ausgebildet haben: Die Kuppel steht für die Tradition der École des Beaux-Arts, die Klimadecke für die Tradition der polytechnischen Lehranstalt.

Offenkundig betreten wir eine Konfliktzone, wenn wir im Rahmen des Interdisziplinären Labors Bild Wissen Gestaltung von Gestaltung als Synthese sprechen. Denn anders als der Begriff im ersten Moment glauben lassen könnte, ist Synthese hier nicht einfach eine harmonisch-friedliche Zusammenführung verschiedener Dinge, sondern setzt ein Verhandeln von widersprüchlichen, teilweise entgegengesetzten Kräften voraus.

Ich möchte hier die These aufstellen, dass diese Synthese gerade dann produktiv ist, wenn wir den Eigensinn der unterschiedlichen Wissenskulturen und Arbeitsweisen - nämlich von Naturwissenschaften, Geisteswissenschaften und Entwurfsdisziplinen - stärken und in ein dialogisches Spannungsfeld setzen, anstatt auf Basis durchaus bestehender Ähnlichkeiten eine große Gemeinsamkeit zu konstruieren versuchen. Doch genau darauf zielt ein guter Teil der über 50-jährigen Debatte des Design Research, die es zu hinterfragen gilt. $^{2}$

1 14. Architekturbiennale Venedig, vom 7. Juni bis 23. November 2014.

2 Einen guten Überblick über die Debatte gibt Mareis 2011. Zur Syntheseidee exemplarisch Schäffner 2010. 
Die Suche nach Verwandtschaften, gar Gemeinsamkeiten zwischen Wissenschaft und Gestaltung ist durchaus erhellend. So ist die Destruktion der Fiktion einer reinen, strengen Wissenschaft durch Wissenschaftstheoretiker wie Paul Feyerabend ${ }^{3}$ oder Hans-Jörg Rheinberger ${ }^{4}$ ebenso gewinnbringend wie die Demontage der Idee des Künstlergenies und das Öffnen der Blackbox der Entwerfenden. ${ }^{5}$

Doch die Betrachtung anderer Kontaktzonen dieser verschiedenen Wissenskulturen offenbart auch grundsätzliche Differenzen. Ein wichtiger Teil baulich-architektonischer Erneuerung, wenn nicht gar ihr wesentlicher Motor, ist die technische Entwicklung. Hier wird das Wissen der Naturwissenschaften produktiv gemacht. Und in Patentanmeldungen und Patenten findet die Erfindungsgabe eine den Wissenschaftskulturen entsprechende Formalisierung: Sie zeichnen sich durch Explikation, Dekontextualisierung, Verallgemeinerung, Reproduzierbarkeit, Überprüfbarkeit und Innovationshöhe aus.

Seit Einführung des Patentwesens in Deutschland 1871 wurden allein im Bereich des Bauwesens etwa eine Million Patentanträge gestellt. ${ }^{6}$ Die Patente behandeln spezifische Detailfragen, indem sie sich mit einzelnen isolierten Bauelementen, wie etwa dem Fassadenabstandshalter, befassen (Abb. 2, 3). Es ist die Explosion eines Wissens, welches hochgradig fragmentiert ist. Interessanterweise gibt es auch Gebäudepatente. So haben gerade einige Exponenten der klassischen Avantgarde Entwürfe patentieren lassen (Abb. 4, 5). ${ }^{7}$ Doch diese waren folgenlos, weil sich die Logik des architektonischen Entwerfens - durchaus in Differenz etwa zum Möbeldesign ${ }^{8}$ - nicht in die Wissenskultur des Patentwesens überführen lässt und diese Architekturpatente niemals zur Anwendung kamen.

3 Feyerabend 1976; ders. 1984.

4 Exemplarisch Rheinberger 2001.

5 Exemplarisch Schön 1983; Cross 2007.

6 Für viele technische Ideen erfolgt nicht zuletzt aus Kostengründen lediglich eine Patentanmeldung, ohne diese bis zur Erlangung eines Patentes weiterzuverfolgen. Zugrunde liegt der genannten Anzahl eine eigene Auswertung der Statistiken des Deutschen Patent- und Markenamtes, veröffentl. im Blatt für Patent-, Muster- und Zeichenwesen, bearb. durch Jochen Jürgensen, im Zeitraum von 1914 bis 2012 in Fünfjahresschritten. Die Zeit davor wurde abgeschätzt.

7 Hierzu zählen etwa die Patentierung ganzer Gebäude oder Baustrukturen von Peter Behrens (Aus mehr- und vielgeschossigen Einzelhäusern bestehender Baublock, Patent vom 25. Juni 1930), Walter Gropius (Theaterbau, Patent vom 27. Dezember 1928), Ernst Neufert (Gebäude mit von außen nach innen abgestuften Geschossen, Patent vom 18. August 1934), Heinz Rasch (Gebäude mit einer oder mehreren vertikalen Röhren und an den oberen Enden aufgehängten Geschoßdecken, Patentauslegungsschrift vom 14. Dezember 1961) und Bruno Taut (Bildvorführungsraum für liegende Zuschauer, Patent vom 16. Juli 1921). Alle genannten Patente wurden im Deutschen Reich bzw. in der Bundesrepublik Deutschland angemeldet.

8 Wirksam sind hingegen die Möbelpatente von Architekten, etwa von Mies van der Rohe (Sitzmöbel mit federndem Gestell, Patent vom 21. Dezember 1929, Deutsches Reich). 


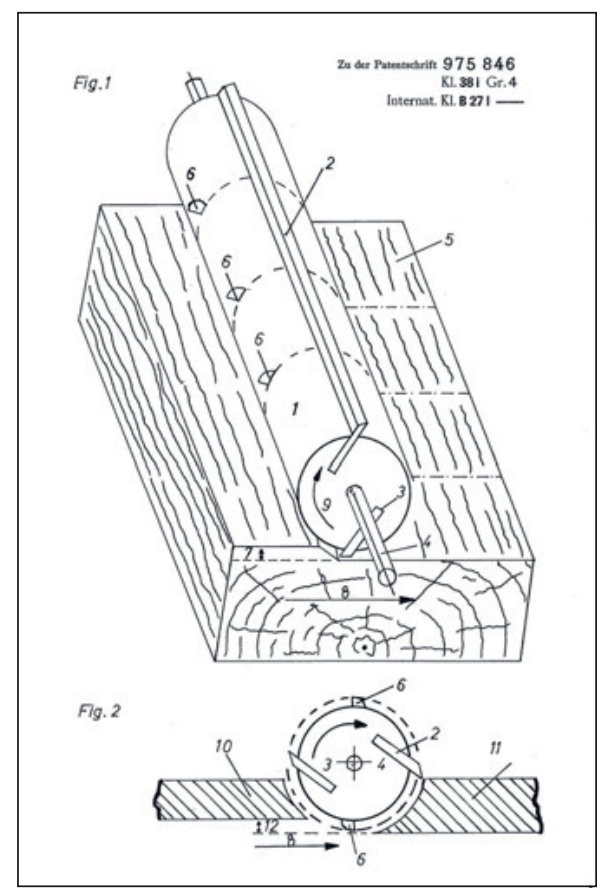

Abb. 2: Max Himmelheber, Holzspanbauteil und Vorrichtung zur Erzeugung seiner Späne, Patent vom 8. Juli 1949.

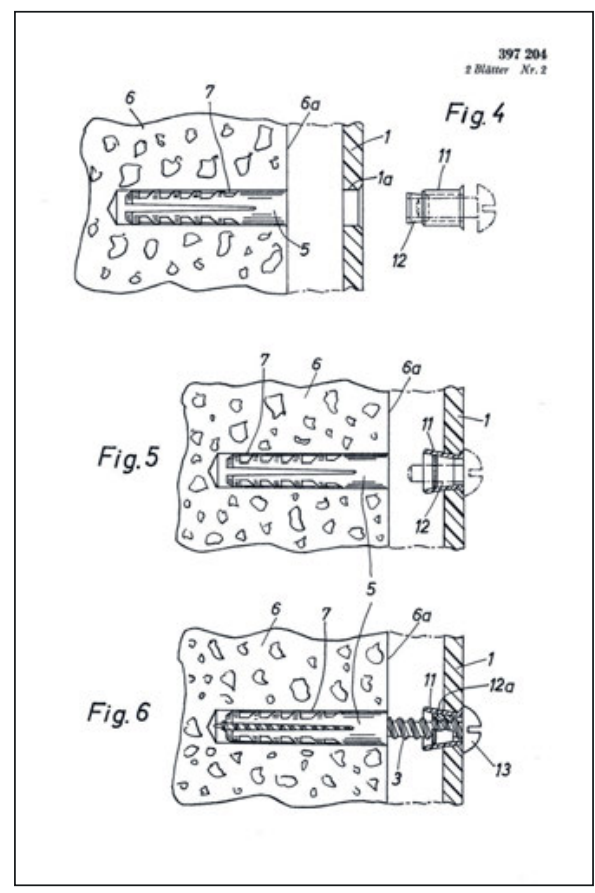

Abb. 3: Artur Fischer, Vorrichtung zur Abstandsbefestigung von Bauteilen, Schweizer Patent vom 15. August 1965.

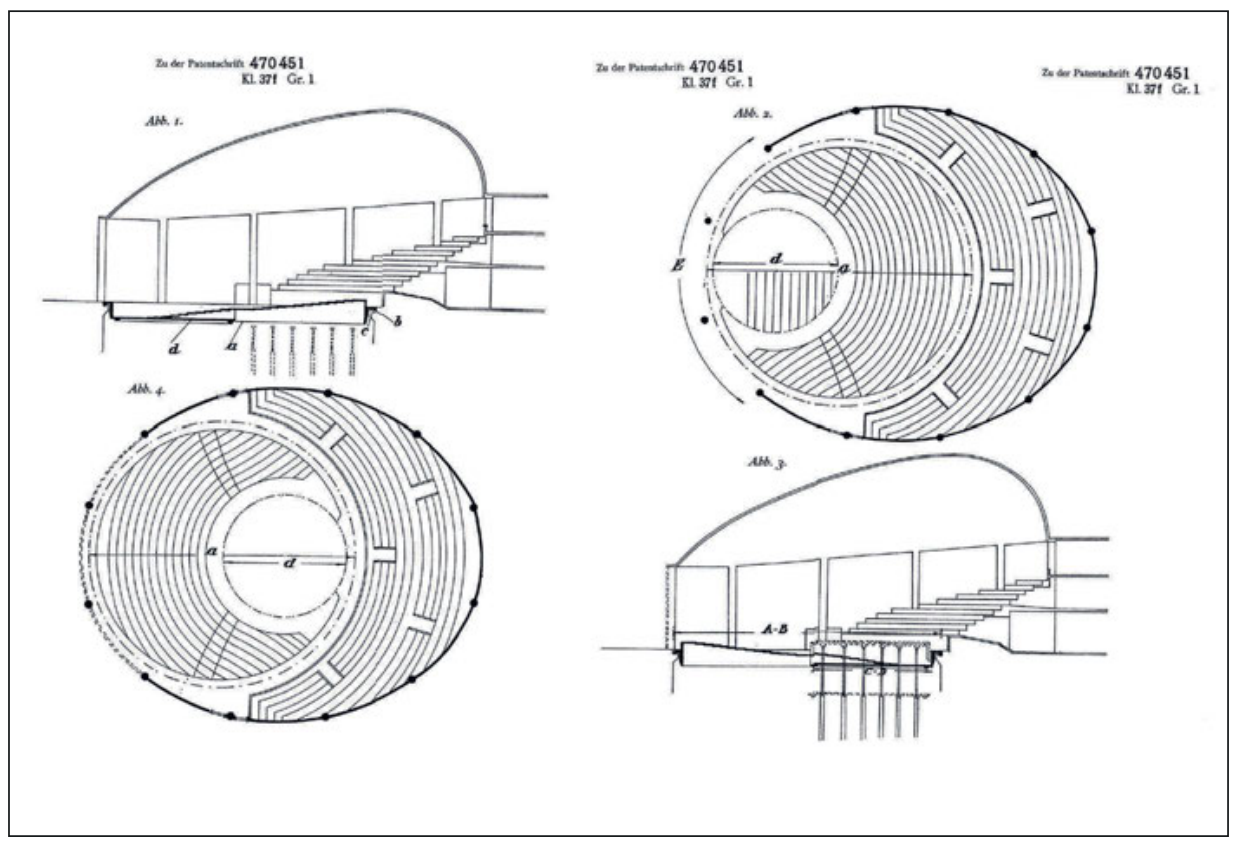

Abb.4: Walter Gropius, Theaterbau (Totaltheater), Patent vom 27. Dezember 1928. 


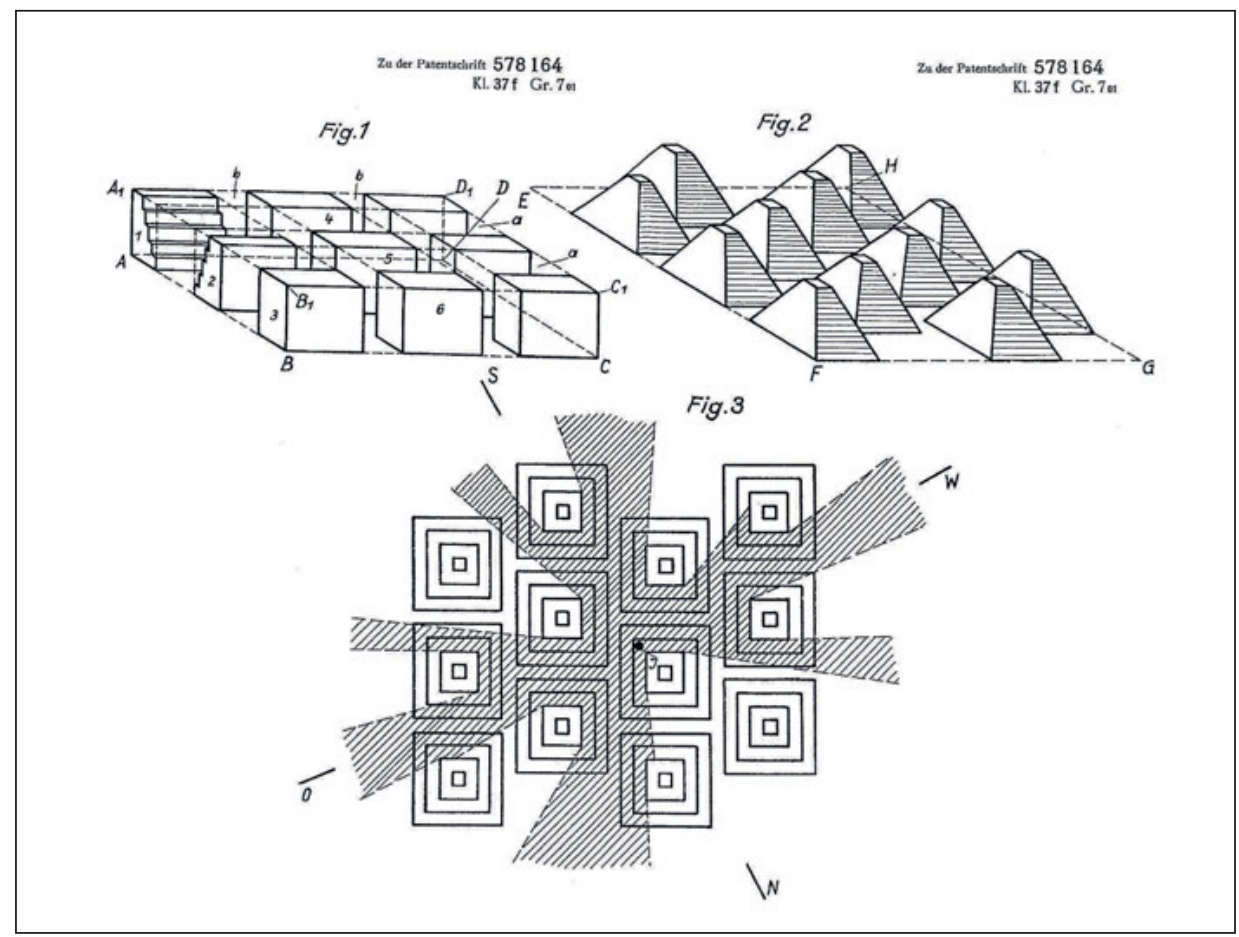

Abb. 5: Peter Behrens, Aus mehr- und vielgeschossigen Einzelhäusern bestehender Baublock, Patent vom 25. Juni 1930.

\section{Nichtwissen}

Das Entwerfen folgt einer anderen Logik: ${ }^{9}$ Es basiert auf Einbildungskraft. ${ }^{10}$ Und hierfür spielt Nichtwissen eine produktive Rolle." In der Vorbereitung zu diesem Vortrag fragte ich einen befreundeten Architekten zu seinem Verständnis, wie das Wissen in das Entwerfen komme, wie Wissenssynthese beim Entwerfen erfolge. Er antwortete: »Die größere Herausforderung für das Entwerfen ist, das angehäufte Wissen wieder loszuwerden. « ${ }^{12}$

Wissen ist nicht neutral, sondern es strukturiert und gestaltet Wahrnehmung und Denken. Erst ein Abstandnehmen von vorhandenem Wissen ermöglicht es, neue Einsichten zu gewinnen.

9 Ohnehin prägt Nichtwissen zunächst die Ausgangslage: Da jeder Entwurf eine Projektion in die Zukunft ist, sind die Kenntnisse der Rahmenbedingungen unsicher und unvollständig. Zugleich sind die Erfolgskriterien eines Entwurfes nicht vorab vollständig und eindeutig bestimmbar, sondern flexibel. Siehe Stephan 2010, 88.

10 Siehe hierzu auch Albert Einstein: »Imagination is more important than knowledge. For knowledge is limited, whereas imagination embraces the entire world, stimulating progress, giving birth to evolution. It is, strictly speaking, a real factor in scientific research.« Einstein 1931, 97.

11 Dabei begrenzt sich das produktive Nichtwissen nicht auf das Entwerfen. Auch die technische Erfindungsgabe folgt nicht notwendigerweise der Logik des Patentwesens. Die Dokumentation des Wissens und damit dessen Überprüfbarkeit, Übertragbarkeit und Archivierung unterscheidet sich eklatant von der Art, wie dieses Wissens entsteht, wie es erzeugt wird.

12 Armand Grüntuch, zit. aus einer E-Mail an den Verf., 19. November 2014. 
Die strategische Bedeutung von Nichtwissen für Erkenntnisprozesse und Handlungen wurde in den letzten zwei Jahrzehnten zunehmend thematisiert und diskutiert, ${ }^{13}$ doch ist diese Denkfigur keineswegs neu.

Zuerst ist in dieser Hinsicht der platonische Sokrates zu nennen, für den echtes Philosophieren nur aus dem Bewusstsein des Nichtwissens möglich ist. ${ }^{14}$ Er unterscheidet zwischen technischem und sittlichem Wissen und sieht die Gefahr in dem Irrglauben von Handwerkern, Politikern und anderen, aus ihrem jeweiligen instrumentellen Fachwissen ließen sich ethische Schlussfolgerungen ableiten. Denn laut Sokrates führt nur bewusstes Nichtwissen zum Erlangen sittlichen Wissens.

Als eine andere Quelle dieses Denkens kann die jüdische Kabbala gesehen werden. Im 16. Jahrhundert formuliert sie die Idee des Zimzum als Schöpfungsmythos: Anfangs ist Gott allgegenwärtig. Durch Selbstkontraktion schafft er einen Hohlraum, ein Nichts, in dem die Welt entsteht. Erst die Abwesenheit schafft Raum und Möglichkeit für die Entstehung des Neuen. ${ }^{15}$ Dass Nichtwissen als künstlerische Strategie wertgeschätzt wird, überrascht wenig. ${ }^{16}$ Die künstlerischen Avantgarden des 20. Jahrhunderts haben immer wieder die Figur der bzw. des Dilettierenden zelebriert. Bemerkenswert aber ist, dass ein Designer wie Otl Aicher, der jegliche Künstlerattitüde ablehnte und eine konsequent gebrauchsorientierte Entwurfsmethode verfolgte, ebenfalls eine wissenskritische Position einnahm. 1991, kurz vor seinem Tod, schrieb er: »Wir sitzen im Gefängnis der eigenen Vernunft. Je mehr wir wissen, um so weniger können wir tun. ${ }^{17}$ Und: »Wir entwerfen, weil wir suchen, nicht weil wir wissen. $\ll^{18}$ Dieses Statement hat umso mehr Gewicht, als Aicher als Mitbegründer der Hochschule für Gestaltung in Ulm einer der zentralen Initiatorinnen und Initiatoren des bis heutige wichtigsten Labors für eine Synthese von Wissenschaft und Gestaltung war.

Aicher ging es nicht darum, Unwissenheit und Dilettantismus zu propagieren. Entwerfen benötigt vielfältiges Wissen. Hierzu gehören insbesondere das langjährige Erfahrungswissen der Entwerferin bzw. des Entwerfers, eine gewissenhafte Analyse der Aufgabenstellung wie auch Wissen über die Möglichkeiten der materiellen Herstellung. Aber der Entwurf ergibt sich nicht aus einer Wissenssynthese, im Gegenteil: Er erfordert einen epistemischen Bruch. ${ }^{19}$ Entwerfende verwerfen, negieren und vernachlässigen vorhandenes Wissen, um zu neuen Einsichten zu kommen. Entwerfen entzieht sich einer deterministischen Arbeitsweise, es ist zugleich unter- und überbestimmt. Für jede Entwurfsaufgabe gibt es eine (wohl unendliche) Vielzahl von möglichen Entwürfen. Dabei würde die Verabsolutierung der verschiedenen, oft sich widersprechenden Entwurfsparameter den Lösungsraum auf ein Nichts zusammenschrumpfen.

Entwürfe sind keine Lösungen, Entwürfe sind Positionierungen.

13 Siehe etwa Düchting/Plüm 2006; Wehling 2006.

14 Siehe Müller 1984; Platon um 380 v. Chr.

15 Siehe Schulte 2014.

16 Siehe Fisher/Fortnum 2013.

17 Aicher 1991a, 189.

18 Aicher 1991, 60.

19 Siehe hierzu auch Petruschat 2011, 14, zum Abbruch von Komplexitätskaskaden mit Bezug auf Herbert Simon. 
Entwürfe beginnen zwar durchaus vergleichbar zur analytischen Forschung mit einer Untersuchung der Gegebenheiten - bei einem Gebäude etwa mit dem vorgesehenen Ort, der gewünschten Nutzung und den vorhandenen Ressourcen, oft auch mit der Analyse vergleichbarer Bauten. Dies erfolgt teils systematisch, teils informell (etwa Besichtigung des Ortes, Gespräche etc.) und alles in allem heuristisch. Doch jeder Entwurf schafft etwas Neues, das sich nicht aus einer gegebenen Situation logisch ergibt, sondern aus einer Bewertung von Alternativen in einem großen Möglichkeitsraum. Im Unterschied zu den Wissenschaften ist das Neue damit keineswegs notwendigerweise das absolut, sondern das für die spezifische Situation Neuartige.

\section{Wissenswachstum versus Wissensverlust}

In Wissenschaften und Technik gehen wir vom Fortschritt aus: Durch Forschung wird immer mehr Wissen produziert. Dieses Wissen vermehrt sich. Laut einschlägigen Schätzungen verdoppelt sich das Wissen der Welt alle hundert oder gar alle zwölf Jahre. ${ }^{20}$ Man spricht von $>$ Wissensexplosion<.

Beim Entwerfen hingegen - so meine ich - kann man nicht von Fortschritt im Sinne von Wachstum sprechen. Der - durchaus erforderliche und vorhandene - > Fortschritt< im Entwerfen ist eher ein Wandel, eine Transformation. Die Innovation, das Entstehen von neuem Wissen, im Entwerfen geht einher mit dem Verlust von altem Wissen. Der Entwurf etwa der Philharmonie von Hans Scharoun oder der Neuen Nationalgalerie von Mies van der Rohe in Berlin steht nicht auf einer höheren Stufe als jener einer gotischen Kathedrale oder eines griechischen Tempels. Zugrunde liegen vielmehr veränderte Entwurfspraktiken. In ihrer Syntheseleistung müssen sie sich mit einem sich wandelnden Kontext transformieren, sie unterliegen einer Koevolution. Dabei werden sie mit zunehmender Komplexität konfrontiert. Mehr und mehr Wissenssorten sind in den Entwurf zu integrieren, mehr und mehr Akteurinnen und Akteure am Entwurfsprozess beteiligt. Gab es in der Renaissance noch eine Einheit von Architekt und Ingenieur, so sind heute an der Planung eines Gebäudes Dutzende von Fachleuten beteiligt - ob aus den Bereichen Haustechnik und Brandschutz, Aufzugs- und Klimaingenieurswesen, Kosten- und Fassadenplanung, um nur einige zu nennen. Der Entwurfsvorgang wird damit selbst weitaus komplexer.

Dabei ist die Rolle der Entwerfenden gefährdet. Es gibt Tendenzen, dass Gestaltende nicht mehr den Entwurf als Ganzes verantworten, sondern zu Spezialistinnen und Spezialisten für ästhetische Fragen (letztendlich Styling) reduziert werden. Die Syntheseleistung wird dann auf andere Personen, Strukturen und Apparate übertragen, welche keine entwurflichen Arbeitsweisen verfolgen, sondern Synthese als methodisch organisiertes Management, als geregelte Prozessierung von Wissen verstehen. Hierbei werden dann keine Entwurfsalternativen mehr verhandelt, sondern die in Software wie dem Building Information Modeling eingeschriebenen

20 Siehe Stuhlhofer 1983, 169-193, sowie Price 1974, bes. 17. 
Lösungen unhinterfragt perpetuiert. Hier erlebt die Sachzwanglogik der technokratischen Nachkriegsplanungen ihre digitale Wiedergeburt.

\section{Verhandlungszone}

Beim Entwerfen ${ }^{21}$ aber erlaubt die Konkretion der Ideen in Zeichnungen und Modellen das Verhandeln heterogener Einflüsse. Sie macht den Entwurf heterogenen Praktiken und Wissensformen zugänglich, erlaubt eine intuitive und logisch-systematische Herangehensweise, assoziatives und kalkulierendes Arbeiten. ${ }^{22}$

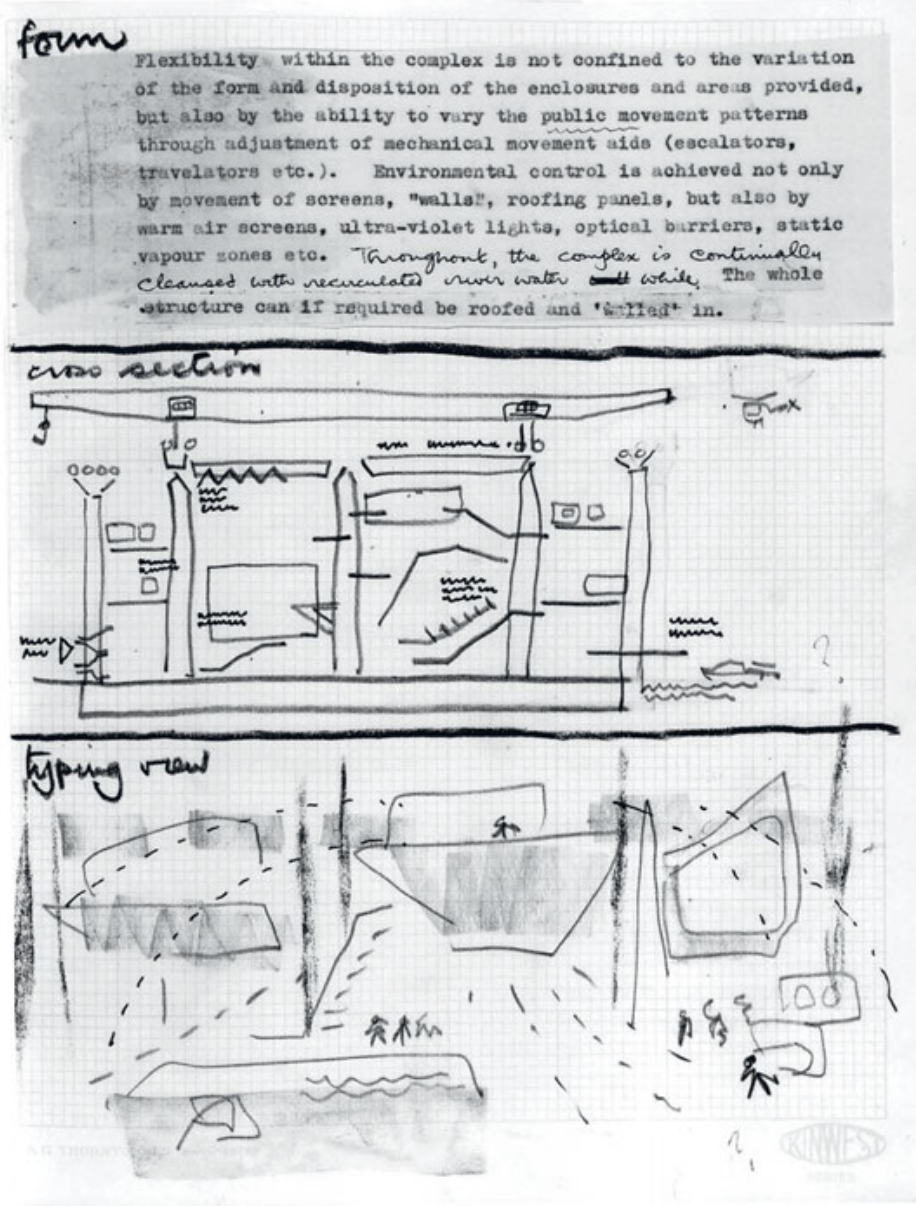

Abb. 6: Cedric Price, Seite aus einem Entwurf von Werbematerial für den Fun Palace, 1963, Tinte, Grafit, Bleistift und Collage auf Papier, $27,8 \times 23 \mathrm{~cm}$.

21 Sicherlich ist es problematisch, von >dem< Entwerfen schlechthin zu sprechen. Es gibt eine große Vielzahl an Arten des Entwerfens, die sich in Vorgehen und Methode unterscheiden. Wenn hier versucht wird, allgemeine Charakteristika des Entwerfens herauszuarbeiten, so werden diese von Fall zu Fall in unterschiedlichem Maße vorzufinden und gelegentlich auch unzutreffend sein.

22 Siehe hierzu auch Stephan 2001, 114; Aicher 1989; ders. 1991, 195. 
Das Modell dient hier nicht der Abbildung einer vorhandenen Realität. Vielmehr stellt es eine zukünftige Möglichkeit dar und erlaubt es, diese zu entwickeln. Das Modell - und damit meine ich hier nicht nur das physische dreidimensionale Modell, sondern auch die Skizze (Abb. 6), die Zeichnung, das digitale Modell - ist das Zentrum des Entwurfsprozesses. Es ist der Verhandlungsort der verschiedenen Wissenssorten, der Abwägung verschiedener Entscheidungskriterien und Entwicklungsmöglichkeiten, der Sichtweisen verschiedener beteiligter Akteurinnen und Akteure, seien es Fachleute aus der Gestaltung, dem Ingenieurswesen und der Herstellung, die Auftraggebenden oder auch die Öffentlichkeit.

Gestaltung findet in den meisten Fällen im Team statt. Entwerfende von Gestaltansätzen stehen im Dialog mit anderen Gestalterinnen und Gestaltern des Studios, die ihren Entwurf rezipieren, kommentieren, verwerfen, verändern, weiterentwickeln etc. (Abb. 7). In diesem Zusammenspiel gibt es einen steten Wechsel des Blicks, einen ständigen Rollenwechsel zwischen Autor_in und Rezipient_in. Abstraktion und Unschärfe von Modell und Zeichnung ermöglichen assoziative Umdeutungen und befördern produktive Missverständnisse. Gerade hierdurch werden sie zum Medium des kreativen Dialogs. Die Externalisierung der Entwurfsidee erlaubt die Distanznahme und Neubewertung der Gestalt und ermöglicht neue Sichtweisen.

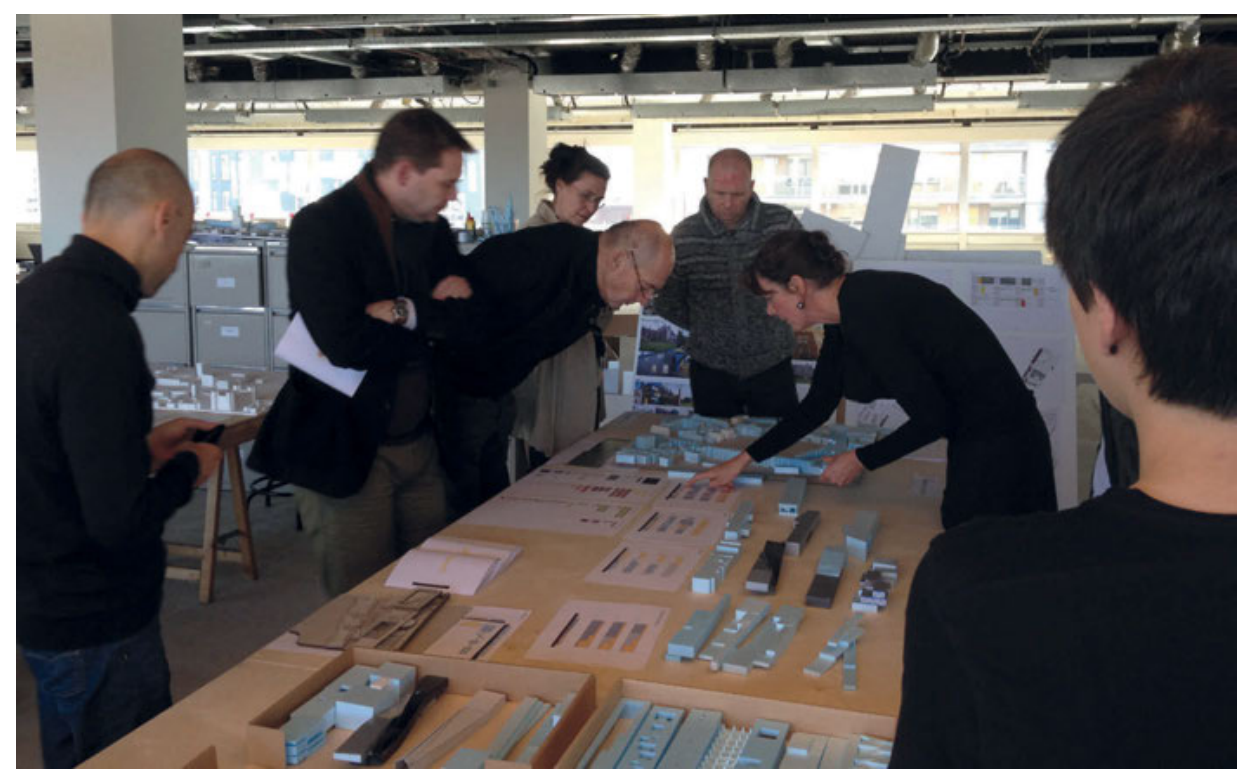

Abb.7: Entwurfsbesprechung im Büro OMA, unter anderen mit Rem Koolhaas (Mitte links).

Dabei ist der Dialog nicht auf das Entwurfsteam begrenzt. Gestaltung ist koproduziert. Auch das Wissen von Fachkolleginnen und -kollegen (etwa bei Wettbewerbjuries), Öffentlichkeit, Bauherr- und Käuferschaft, Nutzenden und anderen fließt in den Prozess mit ein. Zwar entsteht ein Entwurf zuerst einmal im Büro, aber dies ist quasi ein Rohprodukt, welches erst durch diverse Dialoge real wird. Hierbei werden dem Entwurf Bedeutungen und Eigenschaften ein- 
geschrieben, die seitens der Gestaltung nicht bewusst intendiert waren, die aber als Latenz im Objekt enthalten sind. Und diese Zuschreibungen und Projektionen können auch wieder auf den Entwurf selbst rückwirken, seine Gestalt verändern.

Im Entwurf kondensiert sich also das vielfältige Wissen verschiedener Akteurinnen und Akteure. Aber Entwerfen ist keine Synthese vorhandener Wissensbestände. Ein Entwurf ist ein Wurf, er ist gerichtet. Er ist nicht neutral, und er ist nicht alternativlos. Er bewertet und nimmt eine Position ein. Er akkumuliert nicht nur Wissen, er verwirft es auch. Er ist Ausdruck einer Haltung, eines Selbstverständnisses.

\section{In-der-Welt-Sein}

Im Entwerfen setzt sich der Mensch mit der Welt in Beziehung. Er entwirft sein In-der-Welt-Sein. Der Philosoph Ludwig Wittgenstein entwarf und baute mit dem Architekten Paul Engelmann in den Jahren 1926 bis 1928 für seine Schwester Margarethe Stonborough-Wittgenstein ein Wohnhaus in Wien. Quasi als Resümee dieser Erfahrung schrieb er 1931: »Die Arbeit an der Philosophie ist - wie vielleicht die Arbeit in der Architektur - eigentlich mehr die Arbeit an einem selbst. An der eigenen Auffassung. Daran, wie man die Dinge sieht (und was man von ihnen verlangt). «23 Mit der Entwicklung von Artefakten - ob Architekturen oder andere Gebrauchsobjekte - entwerfen Gestaltende menschliche Verhaltensmodelle, Sichtweisen, Haltungen und Repräsentationen. Die Artefakte sind Werkzeuge, mit denen der Mensch seine Beziehung zur Umwelt gestaltet. Sie sind Manifestationen von Wertvorstellungen und Weltsichten. Entwerfende antizipieren die Positionierung derjenigen Personen zur (Um-)Welt, welche die Artefakte künftig besitzen und benutzen werden. Aus der Fülle an Möglichkeiten realisieren die Artefakte eine Option und verwerfen alle anderen.

Entwerfen basiert - anders als das angeblich wertfreie Wissen - explizit auf Werturteilen. Die Gestalter und Theoretiker der Ulmer Hochschule für Gestaltung Tomás Maldonado und Gui Bonsiepe plädieren für einen offensiven Umgang mit diesen, wenn sie fordern, dass Gestalterinnen und Gestalter auch die Wünsche (den >Bedarf $\iota$ ) nach Produkten mit beeinflussen sollen. Daher sollte »die Funktion des Produktgestalters [...] nicht darin liegen, Ruhe zu bewahren, sondern Unruhe zu stiften « ${ }^{24}$ Ihr ehemaliger Kollege, der Designtheoretiker Horst Rittel, nennt es später »design of desirable state «. ${ }^{25}$ Selbstverständlich setzt er voraus, dass Design nicht objektiv und neutral ist, sondern subjektiv auf den Wertvorstellungen, Überzeugungen, Wünschen und Vorlieben von Gestaltenden basiert. ${ }^{26}$ In der Wertfrage liegt wohl der wichtigste Unterschied zwischen dem Entwerfen und den Wissenschaften, und sie verweist auf die von Sokrates betonte Differenz zwischen instrumentellem und ethischem Wissen.

\footnotetext{
23 Wittgenstein 1984, 472.

24 Madonado/Bonsiepe 1964, 29.

25 Rittel 1988, 1.

26 Siehe ebd., 6 .
} 
Wissenschaftliches und technisches Wissen nimmt kontinuierlich zu. Es ist der Motor einer kontinuierlichen Modernisierung, die vorhandene Zusammenhänge auflöst, zentrifugal expandiert und sich ungerichtet entfaltet. Bisherige Kohärenzen lösen sich auf. Das Wissen und die mit seiner Hilfe produzierten Artefakte sind - wie es etwa am Beispiel der Patente deutlich wurde - zunehmend fragmentiert.

Gestaltung zielt hingegen auf Ganzheiten. Sie ist die kompensatorische Gegenkraft der Modernisierung, ihre kulturelle Reparaturtruppe. Sie bindet die sich entfaltende Komplexität und Fragmentierung wieder in neue Zusammenhänge und Kohärenzen ein. Sie richtet die neuen Möglichkeiten auf wünschenswerte Ziele aus und versucht der Modernisierung einen übergeordneten Sinn einzuschreiben. In Abwandlung eines Zitats des britischen Architekten Cedric Price möchte ich daher enden mit der Frage: »Knowledge Is the Answer, But What Was the Question? « ${ }^{27}$ Je mehr wir allenthalben von der Wissensgesellschaft sprechen, desto drängender wird es sein, eine Antwort auf diese Frage zu finden.

27 Das Originalzitat lautet »Technology Is the Answer, But What Was the Question?«, Titel eines Vortrages von Cedric Price, Price 1979.

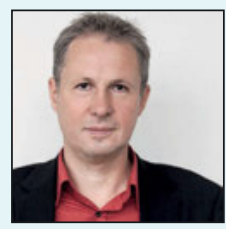

mail@oswalt.de

Associated Investigator

Basisprojekte: Die Anthropozän-Küche, Piktogramme

Disziplin: Architektur

Philipp Oswalt ist Associated Investigator im Exzellenzcluster Bild Wissen Gestaltung. Er lehrt seit 2006 als Professor für Architekturtheorie und Entwerfen an der Universität Kassel und war von 2009 bis 2014 Direktor der Stiftung Bauhaus Dessau. Von 2002 bis 2006 war er Leiter des internationalen Forschungs- und Ausstellungsprojektes Schrumpfende Städte, einem Initiativprojekt der Kulturstiftung des Bundes. Außerdem leitete er von 2001 bis 2003 gemeinsam mit Klaus Overmeyer das europäische Forschungsprojekt Urban Catalyst und war Mitinitiator und künstlerischer Koleiter der Initiative Zwischenpalastnutzung im Volkspalast Berlin. 


\section{Literatur}

Aicher, Otl (1989): Architektur und Erkenntnistheorie. In: Lambot, Ian (ed.): Building and Projects of Foster Associates, vol. 2. Berlin: Ernst \& Sohn, S. 154-159.

Aicher, Otl (1991): Die Welt als Entwurf. Berlin: Ernst \& Sohn.

Aicher, Otl (1991a): kulturen des denkens. In: ders.: analog und digital. Hg. v. Vossenkuhl, Wilhelm. Berlin: Ernst \& Sohn, S. $180-189$.

Düchting, Susanne/Plüm, Kerstin (Hg.) (2006): Nichtwissen. I.K.U.D. Zeitschrift für Kunst- und Designwissenschaften, Bd. 2. Essen: Seltmann + Söhne.

Einstein, Albert (1931): Cosmic Religion: With Other Opinions and Aphorisms. New York: Covici-Friede.

Feyerabend, Paul (1976): Wider den Methodenzwang. Frankfurt a. M.: Suhrkamp.

Feyerabend, Paul (1984): Wissenschaft als Kunst. Frankfurt a. M.: Suhrkamp.

Fisher, Elizabeth/Fortnum, Rebecca (2013): On Not Knowing. How Artists Think. London: Black Dog Publishing.

Flusser, Vilém (1994): Vom Subjekt zum Projekt. Menschwerdung. Bensheim/Düsseldorf: Bollmann.

Maldonado, Tomás/Bonsiepe, Gui (1964): Wissenschaft und Gestaltung. In: ulm. Zeitschrift der Hochschule für Gestaltung, Jg.10, Nr.11, S. 10-29.

Mareis, Claudia (2011): Design als Wissenskultur. Interferenzen zwischen Design- und Wissensdiskursen seit 1960 Bielefeld: transcript.

Müller, Gerhard (1984): Nichtwissen, sokratisches. In Ritter, Joachim/Gründer, Karlfried (Hg.): Philosophisches Wörterbuch, Bd.6. Basel/Stuttgart: Schwabe \& Co., S. $836-838$.

Petruschat, Jörg (2011): Wickes Problems. Vortrag im Rahmen der Konferenz Practice Based Research an der Bauhaus Universität Weimar, 2. Dezember.

Platon (um 380 v. Chr.): Apologie des Sokrates - Griechisch/Deutsch. Übers. und hg. v. Fuhrmann, Manfred. Stuttgart: Reclam 1986.
Price, Cedric (1979): Technology Is the Answer, But What Was the Question? Audiobook. London: Pidgeon Audio Visual.

Price, Derek de Solla (1974): Little Science Big Science. Frankfurt a. M.: Suhrkamp.

Rheinberger, Hans-Jörg (2001): Experimentalsysteme und epistemische Dinge. Eine Geschichte der Proteinsynthese im Reagenzglas. Frankfurt a. M.: Suhrkamp.

Rittel, Horst (1988): Reasoning of Designers. Arbeits papier zum International Congress on Planning and Design Theory, Boston, August 1987 (erschienen in Schriftenreihe des Instituts für Grundlagen der Planung [IGP]). Stuttgart: IGP/Universität Stuttgart.

Schäffner, Wolfgang (2010): The Design Turn. Eine wis senschaftliche Revolution im Geiste der Gestaltung. In: Mareis, Claudia/Joost, Gesche/Kimpel, Kora (Hg.): Entwerfen - Wissen - Produzieren. Designforschung im Anwendungskontext. Bielefeld: transcript, S. 33-45.

Schön, Donald (1983). The Reflective Practitioner: How Professionals Think in Action. New York: Basic Books.

Schulte, Christoph (2014): Zimzum: Gott und Weltursprung. Berlin: Jüdischer Verlag.

Stephan, Peter F. (2001): Denken am Modell. Gestaltung im Kontext bildender Wissenschaft. In: Bürdek, Bernhard E. (Hg.): Der digitale Wahn. Frankfurt a. M.: Suhrkamp. S. $109-129$.

Stephan, Peter F. (2010): Wissen und Nicht-Wissen im Entwurf. In: Mareis, Claudia/Joost, Gesche/Kimpel, Kora (Hg.): Entwerfen - Wissen - Produzieren. Bielefeld: tran script, S. 81-99.

Stuhlhofer, Franz (1983): Unser Wissen verdoppelt sich alle 100 Jahre. Grundlegung einer »Wissensmessung «. In: Berichte zur Wissenschaftsgeschichte, Bd. 6, S. 169-193.

Wehling, Peter (2006): Im Schatten des Wissens: Pers pektiven der Soziologie des Nichtwissens. Konstanz: UVK Verlagsgesellschaft.

Wittgenstein, Ludwig (1984): Bemerkungen über die Farben. Über Gewissheit. Zettel. Vermischte Bemerkungen. Werkausgabe, Bd. 8. Hg.v. Anscombe, Gertrude Elisabeth Margaret/Wright, Georg Henrik von. Frankfurt a. M.: Suhrkamp. 


\section{Entwerfen - Verwerfen}

\section{Ein reflektierender Werkstattbericht aus dem Interaction Design}

Entwerfen - Verwerfen, so lautet der Titel dieses Beitrags, der sich aus einer sehr persönlichen Perspektive heraus mit den Besonderheiten und Eigenarten eines gestalterischen Entwurfsprozesses im Kontext des Interaction Designs beschäftigt. Vorab ist es mir ein Anliegen, einige Worte darüber zu verlieren, aus welchem Zusammenhang heraus die Idee zu meinem Vortrag entstanden ist.

Die Idee, den Entwurfsprozess selbst zum Thema zu machen, kam wie der Gedanke an diesen Titel sehr spontan. Man könnte auch fast sagen: aus einem Reflex heraus. Denn was uns Gestalter_innen hier am Exzellenzcluster Bild Wissen Gestaltung in der Vergangenheit häufiger beschäftigte und womit wir lernen mussten umzugehen, war der Umstand, dass unsere Vorgehensweisen und die daraus resultierenden Arbeitsprozesse für Außenstehende zum Teil nur schwer nachzuvollziehen waren, was nicht immer, aber doch mitunter zu einer gewissen Irritation und Verwunderung auf beiden Seiten führte.

Daher habe ich mir mit diesem Beitrag das Ziel gesetzt, durch die Offenlegung meines eigenen Arbeitsprozesses eine Form der Transparenz herzustellen, die den Zugang zu kreativen Arbeitsweisen erleichtern soll. Daneben möchte ich ganz allgemein zu einem besseren Verständnis für Gestaltung im Kontext von Interaction Design beitragen. Denn wie die bisherige Erfahrung zeigt, verbirgt sich hinter dieser Disziplin für den Einen oder Anderen bislang noch ein Fragezeichen. 
Dabei erhebe ich mit meinen Ausführungen selbstverständlich keinen Anspruch auf Allgemeingültigkeit. Es ist davon auszugehen, dass jede_r Gestalter_in im Laufe der Zeit aufgrund ihrer bzw. seiner persönlichen Biografie und individuellen Arbeitserfahrung ganz eigenständige Abläufe und Vorlieben für bestimmte Entwurfsmethoden und Arbeitsrhythmen entwickelt, die für sie oder ihn charakteristisch sein mögen und sich letztendlich in der Qualität und Ausformung des Entwurfs selbst ausdrücken. Jedoch, so meine Vermutung, verbergen sich hinter all diesen Variationen zumindest auf struktureller Ebene Gemeinsamkeiten, die es im Folgenden herauszuarbeiten gilt.

Ein solcher strukturgebender Mechanismus, der sich höchstwahrscheinlich in allen kreativen Gestaltungsprozessen identifizieren lässt, besteht im Akt des Verwerfens. Die prinzipielle Möglichkeit, eine Idee jederzeit wieder verwerfen zu dürfen und dies auch zu können, scheint für den Prozess selbst überlebenswichtig zu sein. Denn diese Möglichkeit dient einerseits dazu, den Entwurf gedanklich offen und flexibel zu halten, ihn dadurch aber gleichzeitig in seine Konkretion zu treiben. Das Verwerfen wird damit zu einem ständigen Begleiter innerhalb des Prozesses, wobei es dazu dient, Ordnung und Klarheit in den diffusen Nebel aus unüberschaubaren Unsicherheiten zu bringen, die mit jedem Schritt gleichzeitig mehr wie auch weniger werden. Anhand eines Beispiels aus meiner eigenen Entwurfspraxis möchte ich nun diesen Vorgang und dessen Bedeutung verdeutlichen.

Bevor ich damit beginne, erscheint es allerdings sinnvoll, meinen Erläuterungen eine kurze Einführung in das Interaction Design voranzustellen.

Als Interaction Designerin beschäftige ich mich mit dem Verhältnis und der Beziehung zwischen der physischen Welt der materiellen Dinge und unmittelbaren körperlichen Erfahrungen auf der einen und der Welt der digital verarbeiteten und gespeicherten Daten auf der anderen Seite. Dieses Verhältnis gilt es zuerst einmal in seiner Qualität zu verstehen; dabei spielt insbesondere die Perspektive, die der Mensch in diesem Gefüge einnimmt, eine entscheidende Rolle. Folglich rückt auch das menschliche Individuum, das im Folgenden als Nutzerin oder Nutzer bezeichnet wird, mit seinen Eigenarten und seiner für den jeweiligen Fall relevanten Lebenswelt in den Mittelpunkt der Gestaltung. Eine besondere Herausforderung besteht nun darin, die spezifischen Qualitäten beider Welten, also der digitalen und der physischen, herauszuarbeiten und derart aufeinander abzustimmen, dass sie sich entsprechend ihrer Funktion sinnvoll und vor allem angemessen ergänzen.

Das bringt für diese Art des Entwurfsprozesses einige Besonderheiten mit sich, die sich zumindest in ihrer Beschaffenheit von anderen Entwurfskontexten unterscheiden.

Um Interaktionen gestalten zu können, müssen innerhalb eines Entwurfsprozesses testweise Zustände hergestellt werden, anhand derer diese Interaktionen tatsächlich durchgespielt und erfahrbar gemacht werden können. Es werden also im Grunde Erfahrungen bzw. das Erleben einer Interaktion prototypisiert. Dies hat jedoch zur Konsequenz, dass der entscheidende Teil des Prototyps, also die Ausformulierung der Interaktion, materiell nicht greifbar, sondern nur im Moment des Durchspielens und Erlebens ebendieser Interaktion existiert. Hinzu kommt, 
dass im Hintergrund ein digitaler Prozess in Form eines Programmcodes abläuft, der ebenfalls nur durch seine Durchführung erfahrbar wird. Um also auf die > Form< einer Interaktion Einfluss nehmen zu können, müssen, vereinfacht gesprochen, eine oder mehrere Zeilen im Code verändert werden. Im Gegensatz dazu könnte auf ein Modell aus Pappe oder Schaum direkt eingewirkt werden und dessen Form durch Falten, Schneiden oder Feilen verändert werden.

Dieser besondere Umstand macht den Entwurfsprozess für Außenstehende noch abstrakter, als er ohnehin schon ist; daher sollen nun entlang eines konkreten Entwurfskonzepts, das sich mit dem Problem der Integration divergenter Arbeitsmedien befasst, die einzelnen Entwurfsschritte sichtbar gemacht werden. Dieses Konzept ist eingebettet in eine übergeordnete Fragestellung, die sich grundsätzlich mit dem kollaborativen Arbeiten an der Schnittstelle physisch - digital auseinandersetzt. Hierzu habe ich in der Vergangenheit bereits vier Gestaltungskonzepte ausgearbeitet, die sich mit der Integration von und der Interaktion mit physischen Arbeitsmaterialien an der Schnittstelle von digitaler und analoger Arbeitsumgebung beschäftigen.

In diesem Fall handelt es sich um eine typische Schreibtischsituation - sie dürfte allen bekannt sein, die sich digitaler Arbeitsgeräte wie stationärer Computer, mobiler Notebooks oder Tablets bedienen (Abb. 1). Zu sehen ist hier ein aufgeklapptes Notebook, an dem gearbeitet wird und um das sich diverse physische (analoge) Arbeitsmaterialien wie Bücher, Skizzen und Notizen gruppieren, die alle auf verschiedene Art und Weise mit den auf dem Bildschirm dargestellten digitalen Inhalten zusammenhängen und zueinander in Beziehung stehen. Ziel dieses Konzepts ist es nun, eine möglichst schnelle und einfache Methode zu gestalten, um all diese physischen Dinge zu erfassen und sie in einen sinnvollen Zusammenhang mit den digital vorliegenden Daten zu bringen, ohne dabei aber den konzentrierten Arbeitsfluss unnötig zu irritieren. Die

Abb. 1: Arbeitsplatz einer wissenschaftlichen Mitarbeiterin in der Grimm-Bibliothek, Berlin.

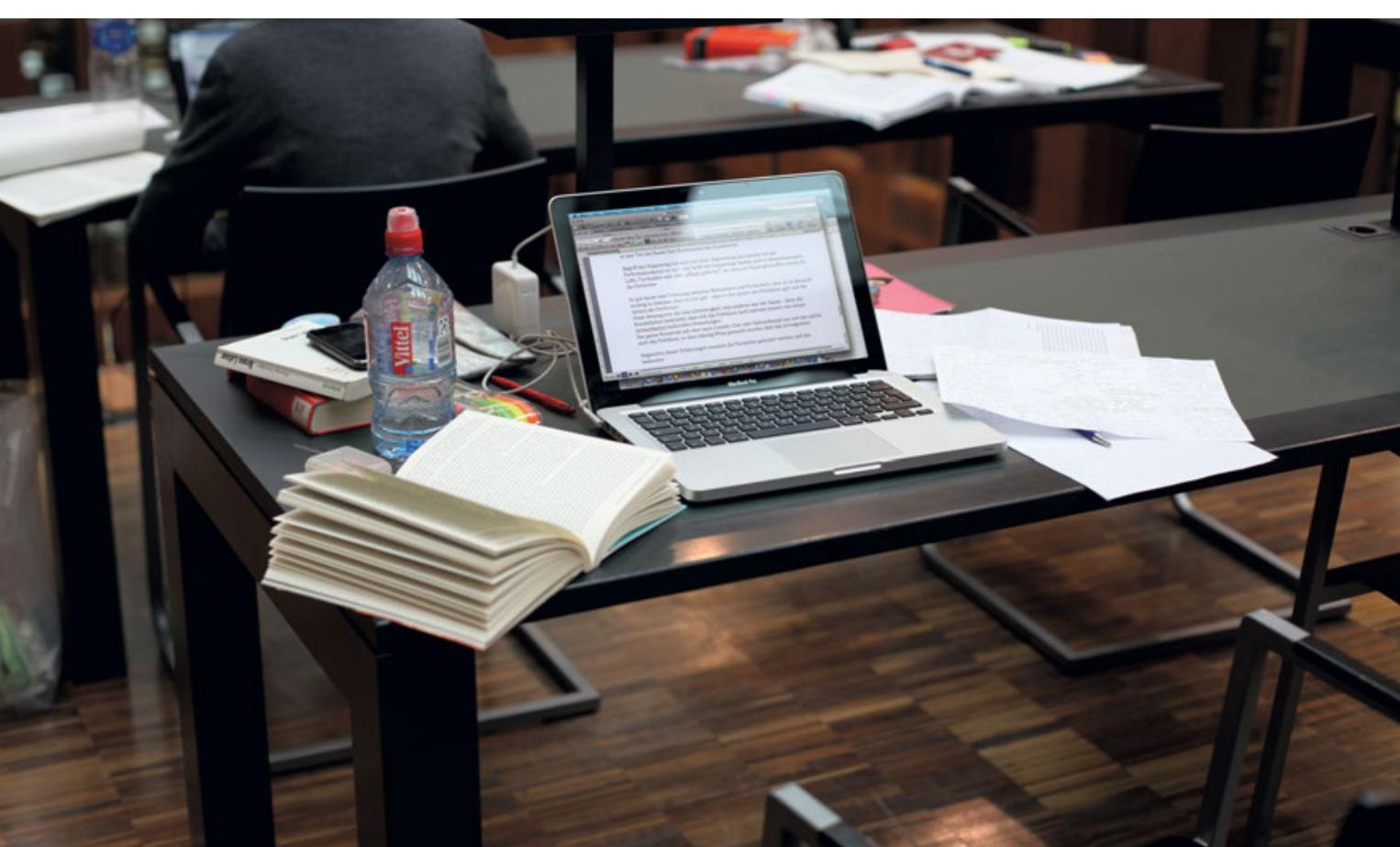


Erfassung der physischen Artefakte soll sich, wenn möglich, unauffällig in die physische Arbeitsumgebung integrieren. Die Interaktion im physischen Raum soll über ein projiziertes Interface stattfinden, welches diese Station unabhängig von seinem Ort machen würde.

Zu Beginn des Projekts steht natürlich eine Recherche, denn wir - das sind im übrigen Tom Brewe, der als studentische Hilfskraft bei uns arbeitet, und ich - sind nicht die Ersten, welche die Möglichkeiten von projizierten Interfaces mithilfe der Beamer-Technologie ausloten. An dieser Stelle sei auf die Forschungsarbeiten von Mark Weiser, die im Rahmen des Konzepts zu Ubiquitous Computing ${ }^{1}$ entstanden sind, verwiesen sowie auf das Project LuminAR ${ }^{2}$ der Forschergruppe Fluid Interfaces am MIT Media Lab in Cambridge, Massachusetts. Im Falle des Ubiquitous Computing steht vor allem die nahtlose und > unsichtbare< Integration rechnergestützter Informationsverarbeitung in intelligente alltägliche Gegenstände im Vordergrund, die als solche nicht mehr als einzelne Computer identifiziert werden. Darauf aufbauend entstand das Konzept des Internet of Things.

Das Project LuminAR knüpft an diese Grundidee an und experimentiert mit Interfaces, die unmittelbar in die physische Arbeitsumgebung eines Nutzers projiziert werden. Die Projektionen sind hier sogar schon in der Lage, den Bewegungen der Nutzer_innen zu folgen. Dies wird durch den Einsatz von steuerbaren gelenkigen Armen ermöglicht. Interessant an projizierten Interfaces ist vor allem deren universelle Einsatzmöglichkeit, denn im Prinzip lassen sich so auf jede beliebige vertikale oder horizontale Fläche in der direkten Umgebung der Nutzer_innen Informationen und Steuerungselemente verorten. Sie sind damit deutlich flexibler als beispielsweise Multitouchtables, die in den meisten Fällen fest an ihren Standort gebunden sind. Ein weiterer Grund, der diesen konzeptuellen Weg bestärkt, liegt in der Miniaturisierung der Beamer-Technologie bei gleichzeitiger Steigerung der Leistung, die einen Einsatz bei normalen Lichtsituationen im Innenraum möglich macht.

Auch aktuelle kommerzielle Projekte im Kontext des erwähnten Internet of Things bieten für unsere Recherche erkenntnisreiche Anknüpfungspunkte. Dabei interessieren wir uns im Speziellen für Anwendungen und Geräte, die ein Lifecapturing ermöglichen, also eine automatisierte Aufzeichnung diverser Daten, die im direkten Umfeld der Nutzerin aufkommen bzw. die sie durch ihr Verhalten selbst produziert.

Geräte dieser Art tragen Nutzer_innen entweder als Wearables in Form von Uhren, Armbändern, Ansteckern oder Kleidungsstücken direkt am Körper oder verwenden hierzu Applikationen auf ihrem Smartphone. In diesem Fall übernehmen Sensoren des Smartphones die Aufzeichnung der Bewegungen und Aktivitäten des Nutzers.

Erwähnenswert sind hier vor allem die beiden Geräte Autographer und Memoto. In beiden Fällen handelt es sich um kleine unauffällige Kameras, die nach unterschiedlichen Parametern automatisch Bilder aus der Perspektive und der Umgebung der Nutzer_innen aufnehmen. Beide Geräte werden direkt am Körper getragen. Die entstandenen Aufnahmen werden sowohl mit

1 Siehe Weiser 1991.

2 Siehe Linder/Maes 2010. 
einem Zeitcode als auch mit den jeweiligen Geodaten im Moment der Erfassung versehen und können später als Stop-Motion-Film auf dem Computer angesehen werden. Das Bildmaterial, das in diesem Fall entsteht, ist allerdings für unsere Zwecke unbrauchbar.

Sowohl die geringe Auflösung als auch die starke Verzerrung insbesondere der Objekte, die in der Horizontalen vor den Nutzer_innen liegen, entsprechen nicht den Erfordernissen unseres Projekts. Zudem basieren beide Geräte nicht auf einem Open-Source-Framework und lassen sich dadurch nur sehr schwer mit anderen offenen Frameworks verbinden.

Nach unserer Recherche ist klar, dass wir nicht auf bereits vorhandene Systeme zurückgreifen und sie für unsere Zwecke modifizieren können. Wir müssen eine eigene offene Umgebung konzipieren, in der alle Freiheitsgrade gegeben sind. Somit besteht der nächste Schritt in der Suche nach einer hochauflösenden Webcam, die sowohl der Detektierung als auch der Erfassung physischer Objekte dienen und über die zu erfassende Fläche am Arbeitsplatz montiert werden soll.

Mit diesem Weg entscheiden wir uns bewusst gegen ein Wearable, das im Prinzip unabhängig von Raum und Zeit ist, und für eine Verortung an eine bestimmte Arbeitsumgebung, die wiederum einen begrenzten Tätigkeitsbereich definiert.

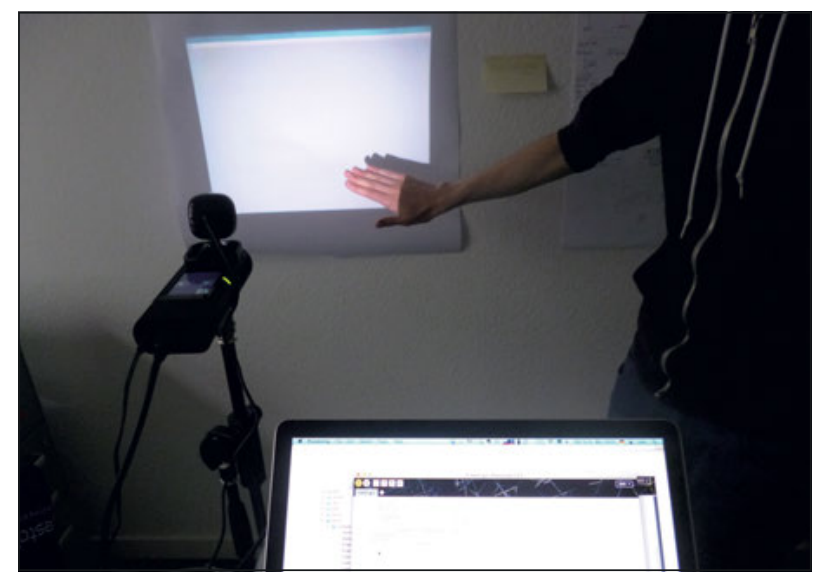

Abb. 2: Mini-Beamer, Webcam,

Programmierumgebung Processing.

Mit diesem ersten Funktionsprototypen haben wir dann letztendlich das Projekt gestartet: Zu sehen sind eine - bisher noch - sehr einfache Webcam, ein Mini-Beamer und die Open-Source-Programmierumgebung Processing (Abb. 2). Der Mini-Beamer projiziert eine Fläche, die einen aktiven Bereich umfasst, in die physische Umgebung. Durch das Verdecken der Fläche mit der Hand wird die Aktion »Erstelle ein Bild mit der Webcam « ausgelöst. Die Webcam übernimmt dabei zunächst einmal die Funktion zu detektieren, dass die entscheidende Fläche, also der Button, aktiviert wurde, und erstellt daraufhin ein Bild.

Das Ziel dieses Aufbaus ist in erster Linie ein Herantasten an das Verfahren der Interaktion mittels einer Projektion. Obwohl der Aufbau sehr einfach ausfällt, führt er schon jetzt zu relevanten Erkenntnissen. Zwar ist eine Interaktion als eine Steuerung des projizierten Interfaces an dieser Stelle schon möglich; doch mutet diese bei Weitem noch nicht so an, wie wir sie uns 
eigentlich vorstellen. Zum einen ist da das Problem, dass die Nutzerin mit ihrer Hand komplett in die Projektion hineingreifen muss, um sie zu kontrollieren. Zum anderen wirkt die Aktion selbst sehr mechanisch und fühlt sich weder intuitiv noch natürlich an.

Wir müssen also sowohl einen Weg finden, komplexe natürliche Gesten zu nutzen, als auch eine Möglichkeit entwickeln, das Interface zu steuern, ohne es dabei ständig zu verdecken. Wie so oft spielt auch in diesem Entwurfsprozess der Zufall oder einfach Glück eine Rolle. Ungefähr zur selben Zeit, als wir mit diesem ersten Prototyp starten, kommt der Leap Motion Controller auf den Markt - ein Eingabegerät, welches in der Lage ist, Handbewegungen und Positionen der einzelnen Finger präzise auszulesen, um dadurch die Verwendung einer komplexen Gestensteuerung zu ermöglichen. Im Gegensatz zur Hardware Microsoft Kinect, die wir ebenfalls für unsere Zwecke in Erwägung ziehen, ist der Leap Motion Controller dafür konzipiert, Handbewegungen in einem Abstand bis zu 1,5 $\mathrm{m}$ zu erfassen. Der Messbereich der Kinect beginnt erst bei einem Abstand von 1,5 m, eine Fähigkeit, die sie für große Bewegungen des Körpers im Raum prädestiniert.

Das beistehende Video Still zeigt, wie es durch die Verwendung des Leap Motion Controllers plötzlich möglich ist, Elemente des Interfaces flüssig zu steuern, ohne dabei die Projektion permanent verdecken zu müssen (Abb.3). Dies mag nun wenig spektakulär anmuten, aber man kann sich kaum vorstellen, wie uns dieses Ergebnis beflügelt hat. Mit einem Schlag wurden beide Probleme einwandfrei gelöst.

Auf dieser Grundlage haben wir eine neue Version des Interfaces entwickelt und den Programmcode dahingehend angepasst. In einem weiteren Video Still ist nun zu sehen, wie physische Objekte positioniert, daraufhin vom Nutzer mit automatisch erzeugten, passenden Schlüsselwörtern getagged und abschließend durch die Durchführung einer Geste (der Nutzer macht eine Faust, als ob er nach dem Objekt greift) erfasst werden (Abb.4).

Zwar sind wir an dieser Stelle dem Ziel schon ziemlich nahe gekommen, jedoch wird auch diese Variante wieder verworfen. Denn nicht sehen, sondern nur in der direkten Interaktion erleben kann man, dass es sich recht seltsam anfühlt, mit dem Zeigefinger über einen Kontrollpunkt kleine Felder hin- und herzubewegen. Zudem müsste die Nutzerin bei der Durchführung dieser Aktion nach wie vor sehr präzise sein, was wiederum gegen die angestrebte Beiläufigkeit und Natürlichkeit der Bedienung spricht.

Folglich wird im nächsten Schritt versucht, die Möglichkeiten des Leap Motion Controllers noch besser auszuschöpfen, um für alle Interaktionen natürlichere Gesten verwenden zu können.

Hierzu werden zuerst einmal Anpassungen des Interfaces vorgenommen. Der Einsatz von großzügigeren Flächen und weniger Optionen dient der Vereinfachung und der Konzentration auf eine deutlich reduzierte Auswahl an Kernfunktionen. Dafür müssen passende natürliche Microinteraktionen definiert werden. An diesem Beispiel soll überprüft werden, wie es wäre, wenn dem Nutzer der Eindruck vermittelt würde, er könne die Schlüsselwörter tatsächlich aufnehmen und auf die Objektfläche werfen. 


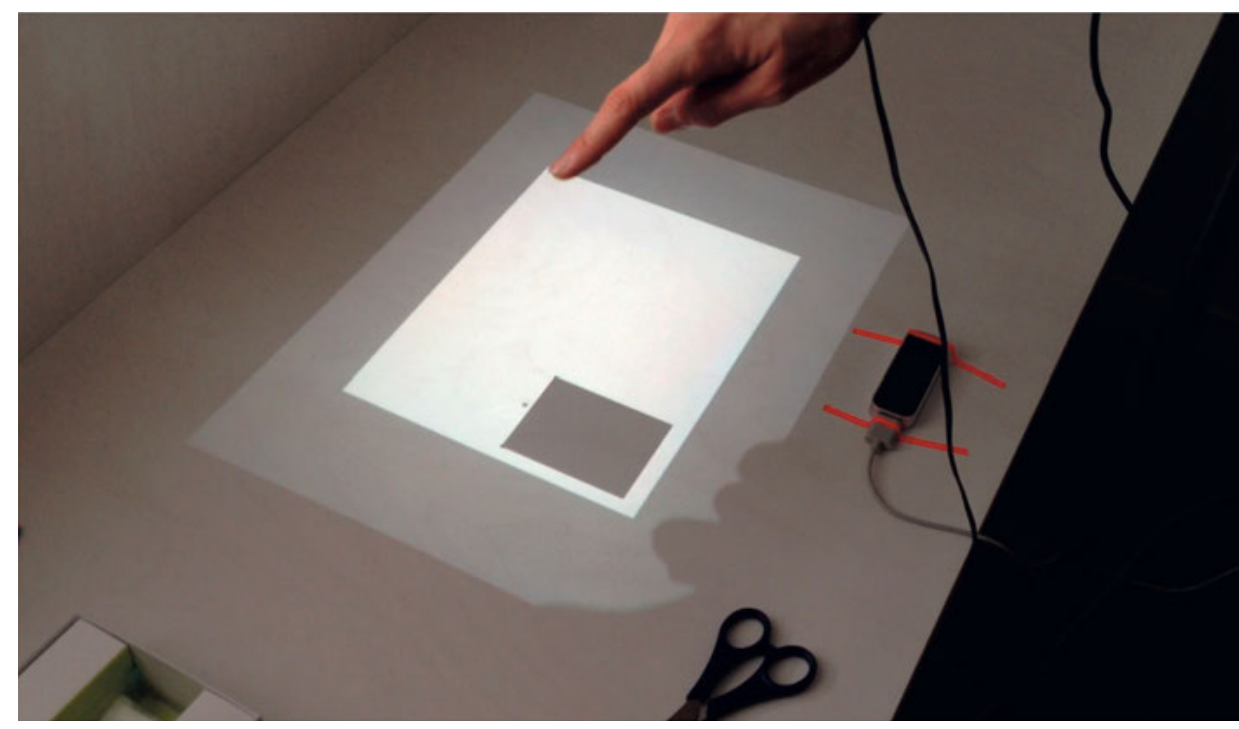

Abb. 3: Leap Motion Controller; Finger steuert Rechteck über Bewegung (Video Still).

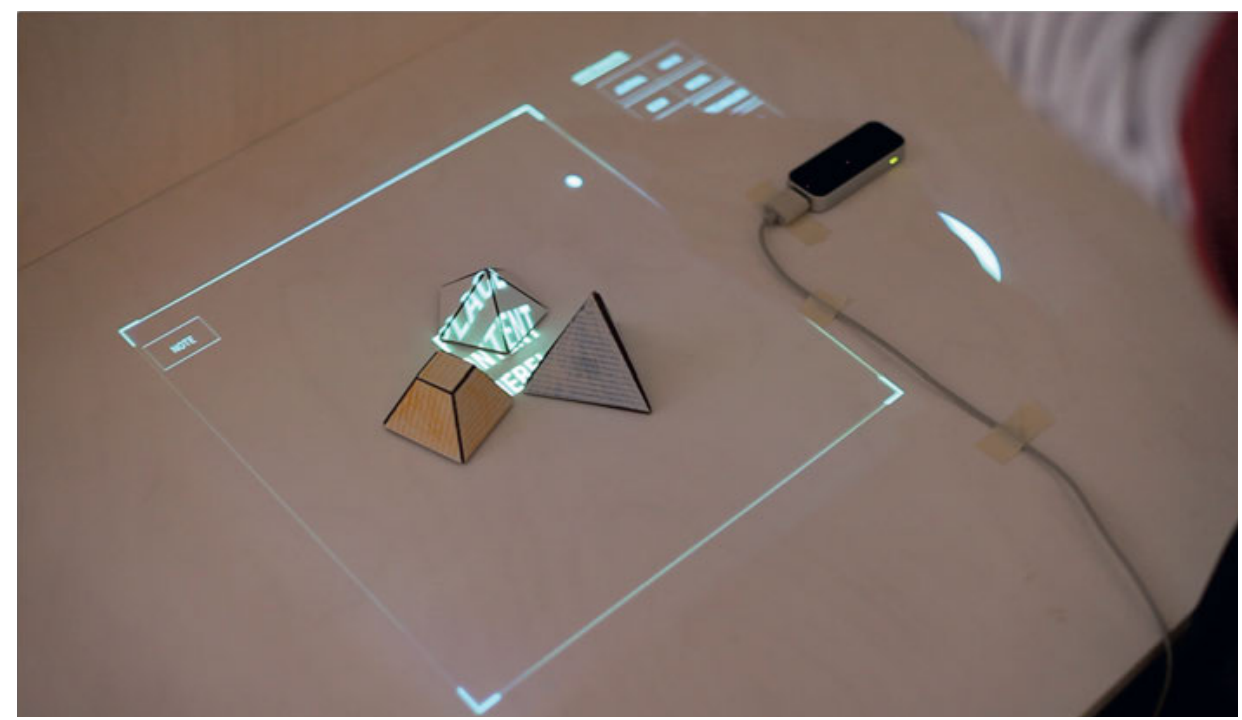

Abb. 4: Leap Motion Controller, phyische Objekte, Schlüsselwörter, projiziertes interface (Video Still).

Auch der vermehrte Einsatz von animierten Transitions könnte der Nutzerin dabei helfen, intuitiver mit dem Interface zu interagieren. Soweit der Plan.

Doch manchmal tut es dem gesamten Entwurf sehr gut, wenn er für einen Moment ruhen darf. Denn was in diesem Fall geschehen ist, ist typisch für den Gestaltungsprozess: Wir haben uns in die Möglichkeiten, die diese neu entdeckte Technologie des Leap Motion Controllers mit sich 
bringt, verliebt und dabei das eigentliche Ziel ein wenig aus den Augen verloren, nämlich die Vision einer beiläufigen und unaufgeregten Interaktion.

Daher ist an dieser Stelle kein Video Still zu sehen. Denn mit zeitlichem Abstand wieder auf das bisherige Stadium geblickt, erscheint dieser Weg viel zu weit vom ursprünglichen Plan entfernt. Die Projektion bzw. das Interface sollen nicht zelebriert werden. Um sie geht es nicht, sondern um die schnelle und minimal disruptive Erfassung eines physischen Objekts. Dazu gehört auch, dass es nicht der Nutzer sein soll, der selbst daran denken muss, etwas zu erfassen; vielmehr soll es die intelligente Arbeitsumgebung sein, die ihn daran erinnert.

Daher bewegt sich das Konzept, an dem derzeit gearbeitet wird, in diese Richtung (Abb. 5). Das konzentrierte Arbeiten der Nutzerin steht dabei noch viel stärker als bisher im Zentrum. Ihre Arbeitsumgebung soll sie auf eine minimalinvasive Art darauf hinweisen, dass sich etwas in der Nähe befindet, das erfasst werden sollte. Dabei richtet sich die Projektion an dem Objekt aus. Es sollte nicht notwendig sein, dieses exakt positionieren zu müssen. Die automatisch erzeugten Schlüsselwörter sollen über beiläufige Gesten auf das Objekt > geschubst< < werden können, und eine Greifgeste schließt am Ende den gesamten Vorgang ab.

Um dieses Ziel zu erreichen, müssen nun einige Umstellungen vorgenommen werden. Unter anderem ist es notwendig, die Programmierumgebung von Processing hin zu Open Frameworks zu verändern, mit der Folge, dass die bisherige Programmierarbeit noch einmal neu beginnen muss. Insgesamt geht dem aktuellen Stadium ein recht großer Akt des Verwerfens voraus, der uns allerdings wie ein Befreiungsschlag vorkommt.

Dieses Beispiel demonstriert, wie sehr das Wissen um die Möglichkeit des Verwerfens hilft, die Dinge gedanklich flexibel und in der Schwebe zu halten.

Der wohl für Gestalter_innen entscheidende Aspekt besteht darin, dass grundsätzlich zu jedem Zeitpunkt des Entwerfens die Möglichkeit des Verwerfens gegeben ist. Daher sollte diese Option nicht als eine potenziell den Entwurf gefährdende Kraft, sondern als eine gestalterische Freiheit aufgefasst werden. Sich von Dingen wieder lösen zu können und dies auch zu dürfen, Ballast abzuwerfen, die Richtung zu wechseln kann für den gesamten Entwurf überlebensnotwendig sein. Denn in den allermeisten Fällen gehen damit eine Radikalisierung, Rückbesinnung und Konzentration auf die wesentlichen Elemente einher, die den Entwurf ausmachen sollen. Die Gewissheit darüber, dass die Elemente auf die eine oder auch ganz andere Art und Weise angeordnet und ausgeführt sein können, bereichert den gesamten Entwurf und bewahrt Gestalter_innen davor, die eigenen Ideen in ihrer Bedeutung überzubewerten und sich zu stark an ihnen festzubeißen. Entwerfen heißt variieren. Verwerfen heißt loslassen. 


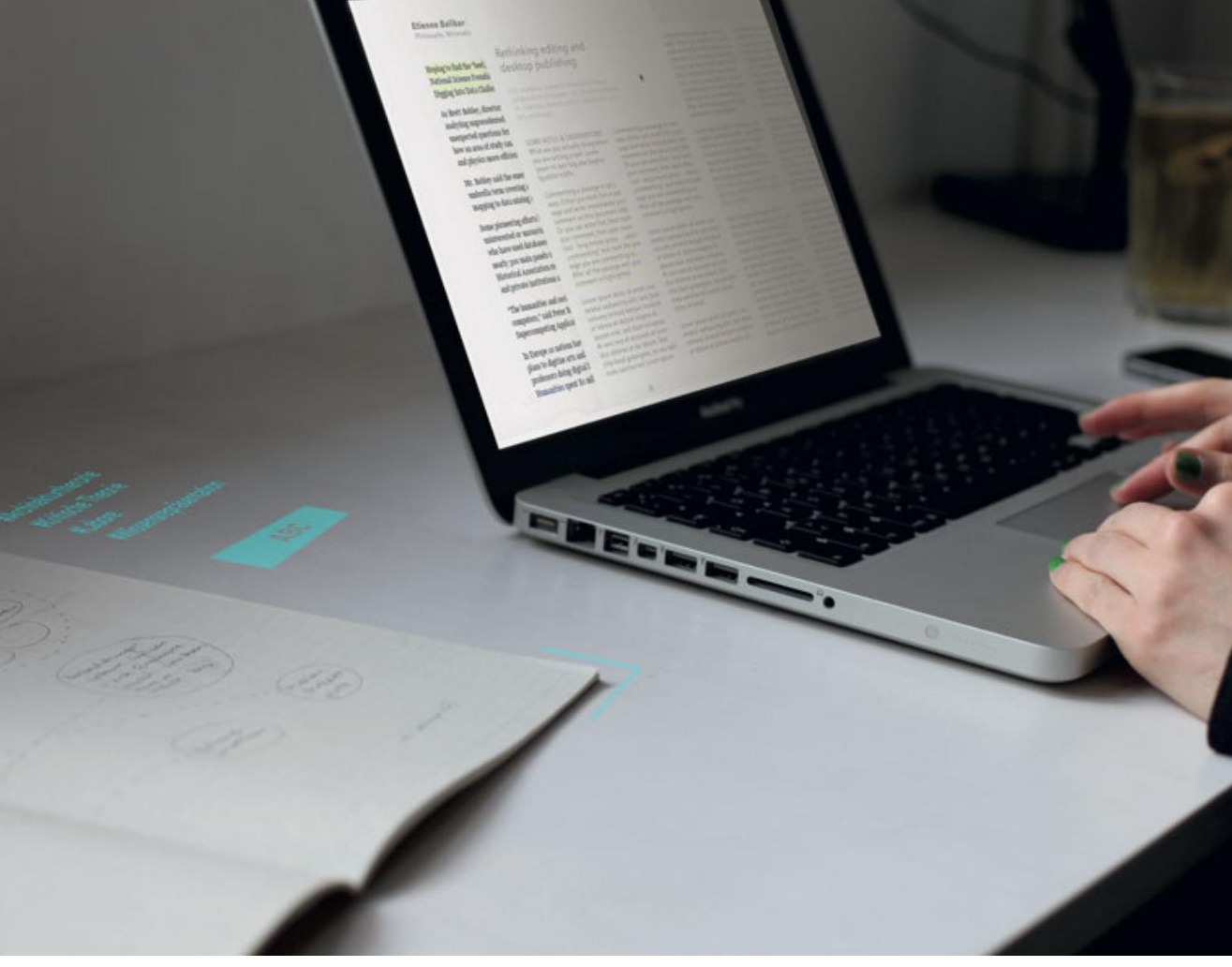

Abb. 5: Simulation der anvisierten Projektion in Verbindung mit der virtuellen Umgebung.

Das vorgestellte Projekt soll darüber hinaus veranschaulichen, dass die Reflexion und der damit einhergehende Erkenntnisgewinn innerhalb des Gestaltungsprozesses vor allem in der Auseinandersetzung mit den entsprechenden Entwurfsmodellen und Prototypen stattfindet, wobei die Herstellung eines Prototypen ebenso wichtig wie das Vertesten desselben ist. Durch seine strukturelle Offenheit spielen auch unvorhergesehene Ereignisse in den Entwurf mit hinein, wodurch es ein Ding der Unmöglichkeit ist, den Prozess in allen Details genauestens zu planen und vorherzusagen.

Gestalter_innen befinden sich demnach innerhalb eines Prozesses gedanklich in der Schwebe, die es ihnen ermöglicht, die einzelnen Elemente immer wieder gegeneinander abzuwägen, sie zu variieren und stets aufs Neue in einen Abgleich mit der dem Entwurf zugrundeliegenden Vision zu bringen. Des Weiteren besteht ihre Aufgabe darin, mit all den Unsicherheiten und noch unkonkreten, weil noch nicht ausformulierten Details im Hinblick auf die Umsetzung zu hantieren und sich dabei Schritt für Schritt an das Ergebnis heranzutasten. Sie müssen in der Lage sein, eine Vorstellung davon zu entwickeln, wie etwas sein könnte, ohne dies im Jetzt schon exakt überprüfen zu können, um darauf aufbauend vorhandene Elemente wieder neu zusammenzusetzen und die Ergebnisse gegebenenfalls wieder zu verwerfen. Mit all diesen gedanklichen Aktionen geht ein permanentes Ein- und Auszoomen in die bzw. aus den Details einher. Somit verwundert es kaum, dass dieser Arbeitsprozess von außen betrachtet sprunghaft, zerfasert, wenn nicht gar chaotisch erscheint. 


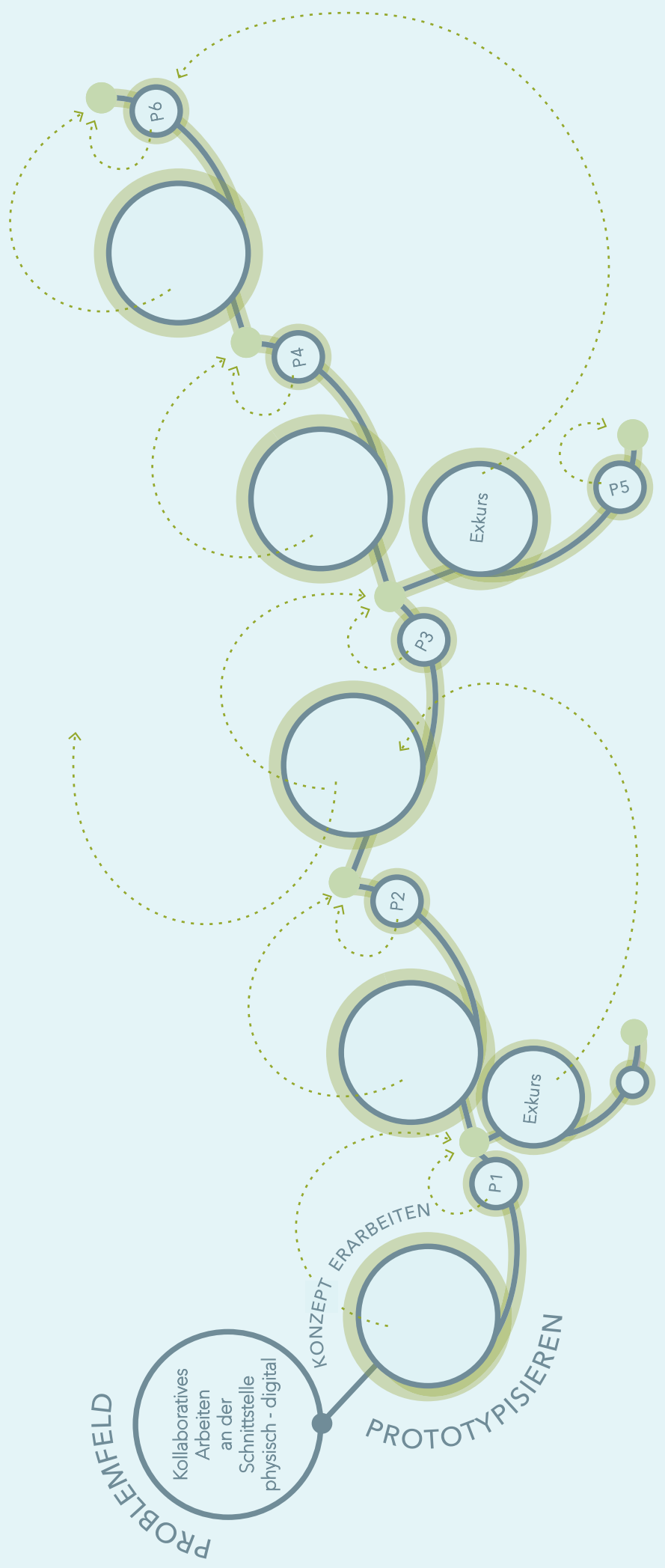

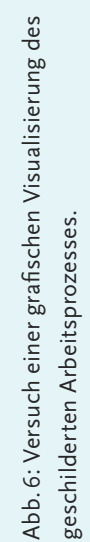


Und dennoch verbirgt sich dahinter eine Abfolge von Einzelschritten, die im Prinzip auf allen >Zoomstufen< identisch ist. Dadurch ergibt sich für den gesamten Prozess eine fraktale Struktur, die auf diesem Wege im Grunde die Erklärung für die Unzugänglichkeit kreativer gestalterischer Prozesse liefert (Abb.6). Denn wie kann in eine ad hoc zu liefernde Erklärung für ein bestimmtes Detail der gesamte Weg dorthin mit allen Alternativen, Irrwegen und Sackgassen miteinfließen? Dies kann nur über das Vertrauen in den gestalterischen Entscheidungsprozess und die Gewissheit darüber erreicht werden, dass jedes einzelne Detail eine Geschichte in diesem Prozess besitzt.

\section{Literatur}

Linder, Natan/Maes, Pattie (2010): LuminAR: Portable Robotic Augmented Reality Interface Design and Prototype. In: Adjunct Proceedings of the 23rd Annual ACM Symposium on User Interface Software and Technology. Association for Computing Machinery (ACM): New York, pp. 395-396. Online: http://fluid.media.mit.edu/sites/ default/files/2010-10-03-luminar_uist10_demo.pdf (last access: 1 April 2015).

Weiser, Marc (1991): The Computer for the $21^{\text {st }}$ Century. In: Scientific American, vol. 265, no. 3, pp. 94-104.

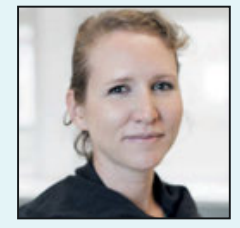

anouk.hoffmeister@hu-berlin.de

Wissenschaftliche Mitarbeiterin

Basisprojekt: Experiment \& Beobachtung

Disziplin: Interaction Design

Anouk-Aimée Hoffmeister hat Sozialwissenschaften in Mannheim und Produktdesign mit Schwerpunkt Interaction Design an der Kunsthochschule Berlin Weißensee studiert. Hier hat sie sich mit dem Gestaltungsprozess des Physical Computings auseinandergesetzt, der sich an der Schnittstelle von digitaler und analoger Welt befindet. Seit 2012 setzt sie ihre Forschungen als Mitarbeiterin im Exzellenzcluster Bild Wissen Gestaltung fort und übernimmt Lehrtätigkeiten an der Kunsthochschule Weißensee. 
Christian Kassung, Sebastian Schwesinger und Christian Seifert

\section{Mit den Haaren hören}

$1832-2014-1897$

\section{Der Seismograf}

Wäre der »academische Bürger an der Hochschule zu München « Lorenz Hengler nicht Priester im katholischen Süddeutschland geworden, so wäre sein Name vermutlich dem vollständigen Vergessen anheim gefallen.' Dabei basiert die Seismografie, also die Aufzeichnung von Erdbeben, zu großen Teilen auf seiner Erfindung, dem Horizontalpendel (Abb. 1). Henglers Idee von 1832 ist bestechend einfach: Weil alle nicht senkrechten Kräfte auf ein gewöhnliches Pendel nur mit dem Sinus einwirken, lässt sich dessen Empfindlichkeit dadurch erhöhen, dass man es in die Horizontale neigt. Das Ergebnis ist ein Instrument, das fähig ist, »jede auch noch so geringe Kraft, welche nicht in paralleler Richtung mit der Schwere wirkt, zu messen ¿ $^{2}$ Doch diese extreme Steigerung der Empfindlichkeit hatte auch zur Folge, dass das Pendel Phänomene registrierte, die Hengler eigentlich nicht sehen oder messen wollte. Sein Pendel stand niemals still, weshalb »alle fremden Kräfte müssen abgehalten werden, besonders Luftzug, oder auch Magnetismus, Elektricität etc. ${ }^{3}$ Und dass man ein Labor natürlich niemals ganz von diesen »fremden Kräfte[n]« befreien kann, wusste auch der Physiker und Spiritist Karl Friedrich Zöllner, 


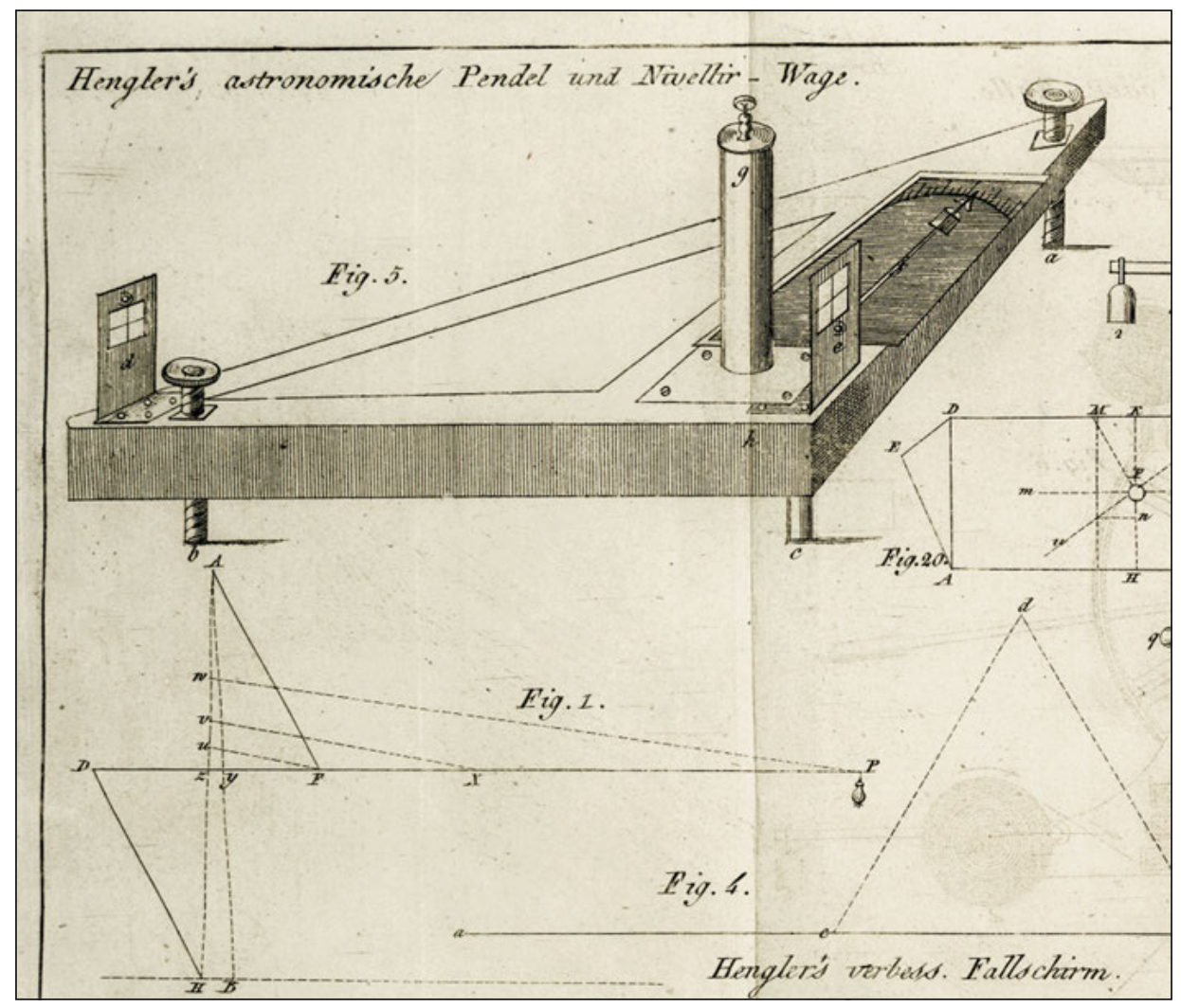

Abb. 1: »Hengler's astronomische Pendel und Nivellir-Wage «.

als er knapp 40 Jahre später Henglers Idee aufgriff, um mittels Horizontalpendel dann eben auch Geistern auf die Spur zu kommen (Abb. 2).

Die sich zwischen Lorenz Hengler und Karl Friedrich Zöllner aufspannende Geschichte ist, ohne dies hier weiter ausführen zu können, paradigmatisch für die mit jedem naturwissenschaftlichen Experiment verbundene Herausforderung: ${ }^{4}$ Je genauer eine Messung ist, umso mehr fallen dabei Daten an, deren Verhältnis zum beobachteten Phänomen unsicher bis problematisch ist. Das Diktum des französischen Philosophen Michel Serres, dass man nur sieht, weil man schlecht sieht, trifft ins Herz der naturwissenschaftlichen Epistemologie. ${ }^{5}$ Ein Experiment muss grundsätzlich zweierlei leisten: möglichst genau messen und möglichst effektiv filtern. Es operiert dabei zwangsläufig an den Grenzen des Wissens.

4 Siehe hierzu ausführlicher Kassung 2007, 238-252.

5 Siehe Serres 1980. 


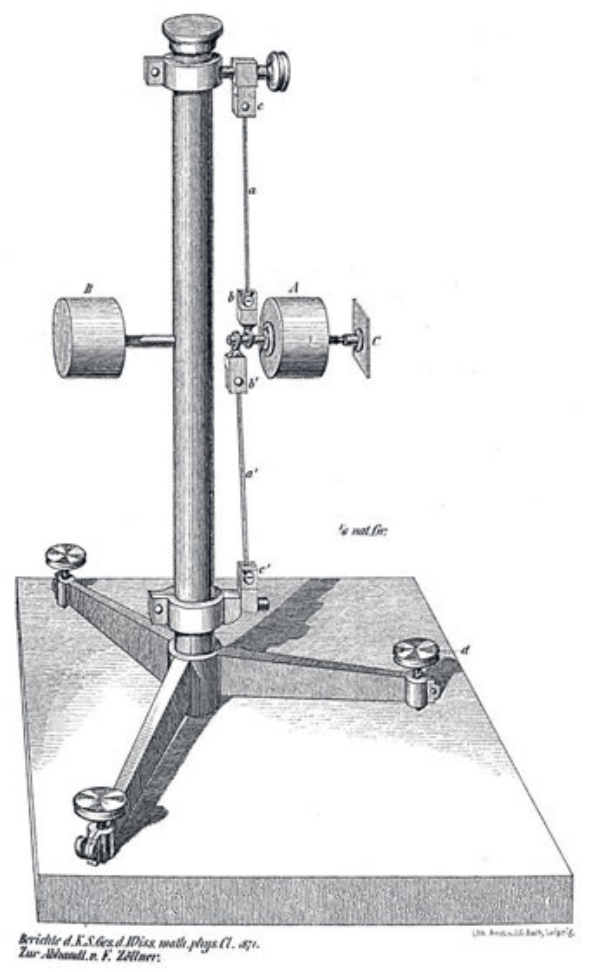

Abb. 2: Horizontalpendel nach Karl F. Zöllner, 1871.

Kommen wir zum Pendel und den mechanischen Kräften zurück, so haben die Experimentalphysiker im 19. Jahrhundert vor allem zwei Strategien entwickelt, um die Genauigkeit einer Messung zu erhöhen. Diesseits des Apparats kann man das Pendel verlängern, den Drehpunkt verschieben, den Zeiger weiter ausschlagen lassen. Je länger der Hebel, umso empfindlicher reagiert das System. Jenseits des Apparats kann man das Pendel in die Horizonale kippen, den Kontaktpunkt verkleinern, die Auflösung erhöhen. Je schmaler der Hebel, umso empfindlicher reagiert das System. Mit einem unendlich dünnen und unendlich langen Stab könnte man alle Geheimnisse dieser Welt ertasten.

Wenn nicht das Tönen der Geheimnisse vom Rauschen des großen Gähnens überlagert wäre, dem Karl Friedrich Zöllner nach 1877 in einer ganzen Reihe von Seancen nachging, während sein immerhin aus einer 2,10 $\mathrm{m}$ langen, dünnen Glasstange bestehendes Horizontalpendel im 12 m tiefen Keller des Universitätsgebäudes eher auf die Studenten im sich darüber befindlichen Auditorium denn auf » elektrische und magnetische Kräfte « reagierte. ${ }^{6}$ Womit sich die Herausforderung der Experimentalphysik zunehmend von der Generierung zur Interpretation der Daten verschob oder eben dem Filter, der jene Differenzen zuallererst erzeugt, denen diskursive Bedeutungen zugesprochen werden können. Die Skalierung der Messanordnung führt demnach ebenso wie deren gänzliche Neugestaltung zu erheblichen Erkenntnisproblemen, indem der Abstand zwischen Rauschen und Signal sowohl kontinuierlich wie auch sprunghaft abnimmt. Die Kakophonie des Rauschens markiert eine Grenze, jenseits derer alles Fühlen, Tasten und Registrieren buchstäblich sinnlos wird. Der Kontaktpunkt lässt sich nicht beliebig verkleinern und die Skalierung nicht beliebig vergrößern. Wer das Pendel zu weit in die Horizontale neigt oder die Abtastnadel seines Apparats zu stark verkleinert, der zeichnet höchstens sich selbst auf, nicht aber das Phänomen. Anders - und polemisch - formuliert: Wer den Versuch wagte, den Kontaktpunkt bis in den atomaren Bereich hin zu verkleinern, der konnte nur entweder als Spiritist enden oder einen Nobelpreis erhalten.

6 Zöllner 1869, 283. 
Bekanntlich ereignete sich Letzteres: ein Nobelpreis. 1986 gelang es der Forschergruppe um den deutschen Physiker Gerd Binnig, ein Mikroskop zu entwickeln, das so empfindlich auf seine Umwelt reagiert, dass sich damit atomare Oberflächen zeilen- und spaltenweise abtasten lassen. Normalerweise wird ein Rasterkraftmikroskop also dazu verwendet, Oberflächen abzutasten. Oder, wie es in der Patentschrift von Gerd Binnig und Heinrich Rohrer heißt, es »wird eine feine Spitze als Abtastelektrode im Abstand von einigen Zehntel Nanometer rasterartig in parallelen Abtastlinien über die Oberfläche einer leitenden Probe geführt «. ${ }^{7}$ Öffentlichkeitswirksam inszeniert wurde diese neue Experimentalpraxis beispielsweise durch IBM-Spielereien mit dem Firmenlogo (Abb. 3) oder dem kleinsten Film der Welt A Boy and His Atom. ${ }^{8}$ Das IBM-Nanologo wurde mit einem Rastertunnelmikroskop hergestellt. Dabei kommt eine Abtastelektrode einer elektrisch leitenden Oberfläche so nahe, dass zwischen Taster und Oberfläche ein sogenannter Tunnelstrom fließt. Mithilfe dieser Kraftwirkung lassen sich einzelne Atome verschieben, wobei die Oberfläche auf das eingeschriebene Logo reagiert: Die wellenartigen Muster stellen die Energiezustände der Elekronen an der Oberfläche dar.

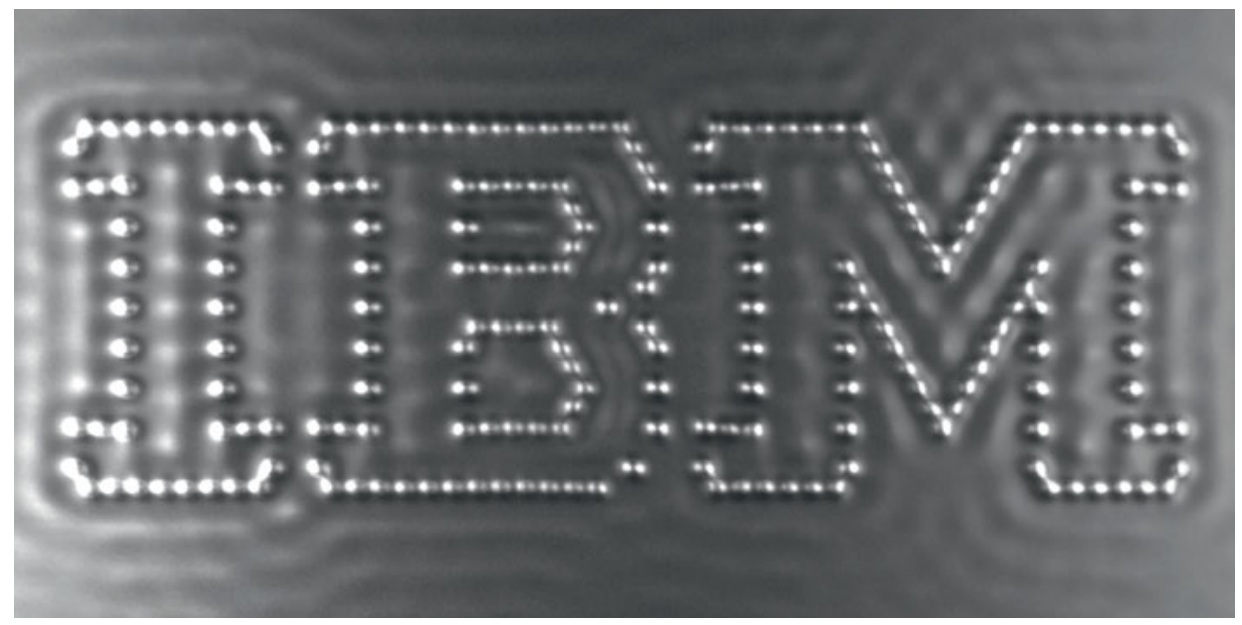

Abb. 3: IBM-Nanologo, Video Still aus A Boy and His Atom, 1:33 min.

Wir können heute also mechanisch sehr viel genauer und tiefer in die Dinge hineinfühlen als hineinsehen. Oder etwas vorsichtiger formuliert: In der Nanowelt der mikroskopischen Tastspitze bzw. des Cantilevers brechen makroskopische Differenzen wie diejenigen zwischen dem Hören, Tasten und Sehen, dem Kontinuierlichen und dem Diskontinuierlichen, dem Analogen

7 Binnig/Rohrer 1979, 1.

8 Online unter: http://www.research.ibm.com/articles/madewithatoms.shtml\#fbid=pKizwGYVHv_ (zuletzt aufgerufen: 6. Januar 2015). 
und dem Digitalen schlichtweg zusammen. Die Skalierung der epistemischen Anordnung aus Membran und Hebel in den mikroskopischen Bereich hinein verwischt dabei aber nicht nur diese begrifflichen Grenzen, sondern sie ermöglicht zugleich eine vertiefte Reflexion dieser Differenzen im Makroskopischen. Die Konzeption des >Hörens < als Abtastung denkt die Wahrnehmung humaner und nichthumaner Akteure zusammen. Was sich derzeit unter dem Label Sonic Materialism als Forschungsperspektive formiert, schließt genau hieran an: ${ }^{9}$ Die Berücksichtigung haptischer Wahrnehmungsmodi erweitert das Hören auf den gesamten Körper, das heißt über das vermeintlich privilegierte decodierende Hören mit den Ohren hinaus. Ebenso etabliert sich in dieser materialbasierten Sichtweite eine skulpturale Vorstellung von Klang unabhängig vom tatsächlichen menschlichen Erleben, die sowohl die Grenzen von Fläche und Form als auch von kontinuierlichem und diskontinuierlichem Ereignis im Medium des Klangs hinterfragt.

Die epistemische Reichweite beim Kollaps dieser gewohnten Differenzen gilt es aber auch durch neue Formen der Experimentalisierung konkret produktiv zu machen. Zurückbezogen auf das Experimentalsystem des Rasterkraftmikroskops lässt sich so beispielsweise der Cantilever mit dem Hören von Oberflächen zusammendenken. Was würde passieren, wenn wir nicht wie bisher Oberflächen abtasten, sondern - indem die Detektionsfunktion der epistemischen Konfiguration aus Membran und Hebel invertiert wird - tasten, wie Oberflächen auf ihre Umwelt reagieren? Aus dem ins Extrem verkleinerten Kontaktpunkt wird eine maximal dünne Kontaktfläche, eine Membran, die schwingen kann. Wir verwandeln das Rasterkraftmikroskop in ein Rasterkraftaudioskop, indem wir einen möglichst kleinen Hebel an einer möglichst dünnen Membran befestigen. Oder metaphorisch gesprochen: Was würde ein Haar hören, wenn wir es auf wenige Nanometer Dicke verkleinerten (Abb.4)?

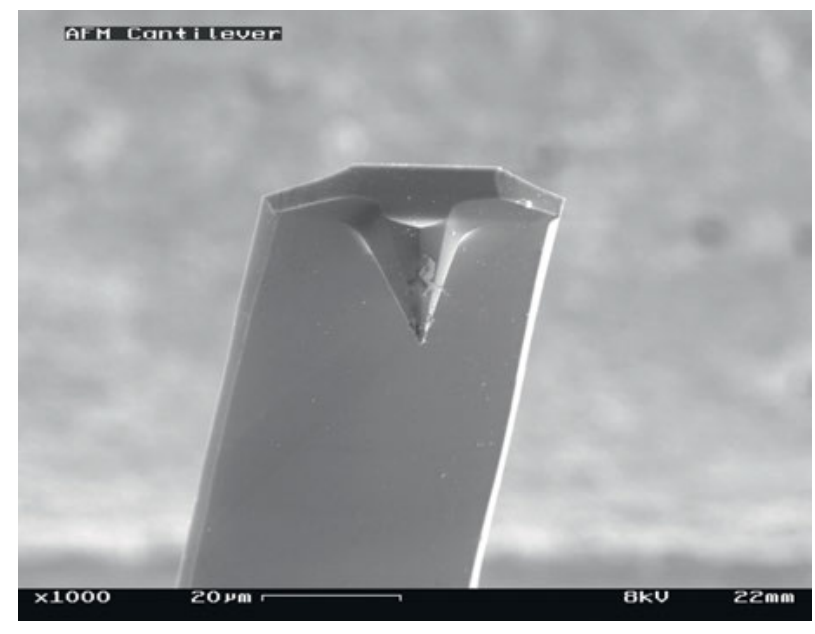

Abb. 4: Cantilever eines Rasterkraftmikroskops (AFM).

9 Vgl. beispielsweise das haptic hearing bei Anahid Kassabian oder den non-cochlear sound bei Will Schrimshaw. 
Im Idealfall ist die aufgehängte Membran eine einatomar dünne Graphenschicht, deren Herstellung jedoch mit einem nicht unerheblichen handwerklichen Aufwand verbunden ist. Sie wird durch Entblättern von einem Graphitblock hergestellt, der auf einem dotierten Siliziumwafer liegt..$^{10}$ Dieser ist mit einer wenige zehn Mikrometer dünnen elektrisch isolierenden Oxidschicht überzogen. Das auf der Oxidschicht präparierte Graphen wird mit einer entsprechenden elektrischen Goldelektrode lithografisch kontaktiert. In einem zweiten lithografischen Prozess wird ein schmaler Graben unter dem Graphen geätzt, sodass die Membran frei und ihren physikalischen Eigenschaften entsprechend schwingen kann." Die zweite Elektrode bildet der dotierte Siliziumwafer selbst. Diese Probe fungiert zugleich als ein Kondensator, das heißt, es kann durch Anlegen einer elektrischen Spannung an die Elektroden eine Kraft auf die Graphenmembran ausgeübt werden, wodurch diese entsprechend dem Spannungsverlauf schwingt. Die Superposition dieser erzwungenen Schwingungen mit den Eigenschwingungen des aufgehängten Graphens sorgt für die notwendige Verstärkung der messbaren Amplituden.

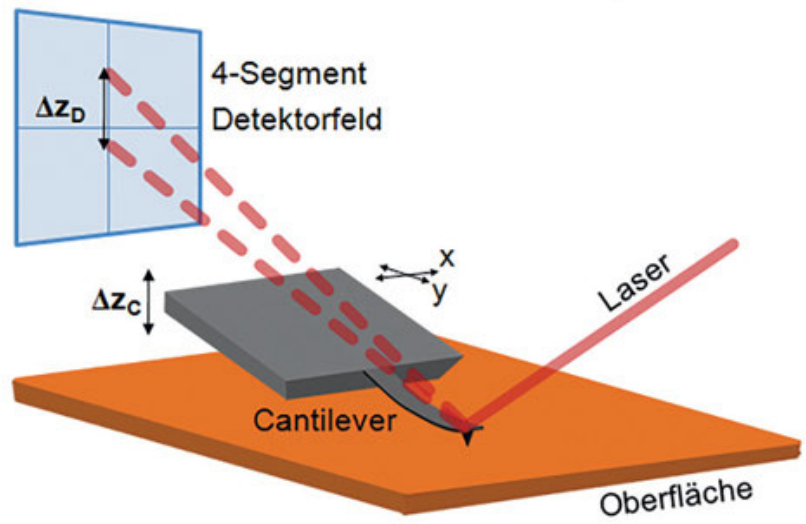

Abb. 5: Prinzip eines Rasterkraftmikroskops: Cantilever, Laser, Detektor.

Als Hebel, der die Bewegungen der Graphenschicht detektiert, dient nun das Rasterkraftmikroskop. Ähnlich wie Zöllner die Bewegungen des Pendels über einen Spiegel vergrößerte, besteht das Rasterkraftmikroskop neben dem Cantilever aus einem Laser, einem aus vier Segmenten bestehenden Detektor und einem Controller (Abb.5). Der Cantilever ist ein wenige Mikrometer langer Schwingarm, an dessen Ende sich eine feine Spitze befindet. Diese hat im besten Fall einen Spitzenradius von wenigen Nanometern und ermöglicht somit eine atomare Auflösung. Die Spitze tastet nun also die Oberfläche ab, wobei sehr komplexe Wechselwirkungskräfte entstehen, was qualitativ hier jedoch vernachlässigt werden kann. Das andere Ende des Cantilevers hängt herstellungsbedingt an einem einige Millimeter großen Körper, um eine bessere Handhabung der Spitze und deren festen Einbau in die Apparatur zu ermöglichen.

10 Erklärtes Verfahren in Eilers 2013.

11 Siehe Garcia-Sánchez 2008, 1399-1403, sowie Eska 1997, 73. 
Wenn also das eine Ende des Schwingarms fixiert ist, muss das andere Ende, an dem sich der Kontaktpunkt befindet, der Oberfläche folgen. Dadurch verändert sich die Position der Spitze kontinuierlich. Ein am Spitzenende reflektierter Laserstrahl überträgt diese Bewegung entsprechend vergrößert auf einen Detektor, welcher das optische Signal in ein elektrisches umwandelt und über einen Controller (der auch zur Steuerung des Rasterkraftmikroskops dient) die aufgearbeiteten Daten ausgibt. Rastert man nun nicht eine Oberfläche ab, sondern lässt den Cantilever auf der Membran ruhen, so wird aus dem Abtasten einer Oberfläche ein Abtasten (oder Hören) mit einer Oberfläche.

Die untenstehende Abbildung zeigt den aktuellen Stand der Laborarbeit, eine nanoskopische Membran durch Entblättern auf einer bereits vorgefertigten Fingerelektrode herzustellen (Abb. 6). Zu sehen ist zunächst eine Höhenbildaufnahme durch ein Rasterkraftmikroskop mit einer Seitenlänge von ca. $20 \times 20 \mu \mathrm{m}$. Klar erkennbar ist eine Differenzierung von Goldschicht, Graben (etwa 2,5 $\mu \mathrm{m}$ breit) und aufliegender Graphenschicht. Betrachtet man die gleiche Situation als Cross Section, also als Profil des Höhenverlaufs, so entsteht ein nicht unerheblicher Interpretationsbedarf des Höhenbildes. Wir sehen von links beginnend eine hohe Goldschicht, den sich ihr anschließenden Graben sowie die nach rechts auslaufende Graphenschicht. Allerdings messen wir in dieser Cross Section eine Schichtdicke des Graphens von etwa 6 bis $7 \mathrm{~nm}$, was ca. 15 bis 18 Lagen entspricht. Dies lässt nur eine Schlussfolgerung zu: Die Graphenschicht liegt auf dem Graben auf, statt wie gewünscht diesen zu überspannen. Offensichtlich ließ sich beim Entblättern bisher noch keine ausreichend dünne Graphenschicht erzeugt. Erst in der Zusammenschau beider Darstellungen wird somit klar, dass die Präparationsmethoden weiter verfeinert werden müssen, um die gewünschten Membranschwingungen detektieren zu können.

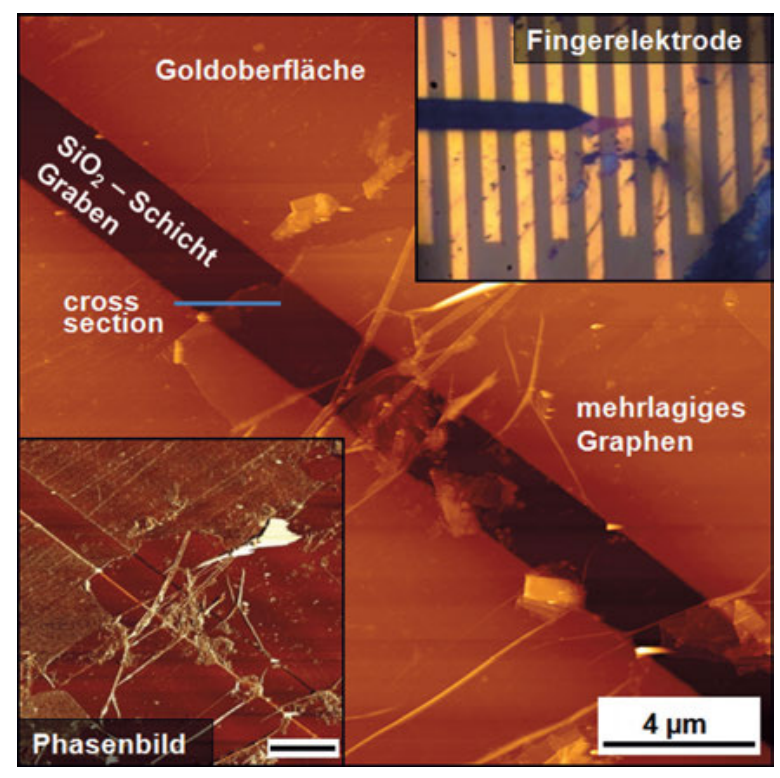

Abb. 6: Höhen- und Phasenbild der Probe. 
Blicken wir nun noch einmal, von diesem konkreten Versuchsaufbau ausgehend, zurück in die Wissenschaftsgeschichte, so führt dies erneut zum Klang zurück, genauer gesagt zur Klang- und Hörforschung. Vor allem beim Hörsinn finden sich Membranen, deren kleinste Bewegungen abgetastet werden. So ist das vielleicht beste Rasterkraftmikroskop schlichtweg unser Ohr. Mit einer Dicke von ungefähr o,1 mm ist das Trommelfell zwar vergleichsweise >klobig<. Trotzdem detektiert es Auslenkungen im Bereich eines Zehnmillionstelmeters, also von 0,01 nm. ${ }^{12}$ Das Trommelfell ist eine Membran, die geringste Druckveränderungen der Umwelt so filtert, dass nur ein bestimmter Frequenzbereich wahrgenommen wird - eben als Klangereignis. Ohne entsprechende physiologische und kognitive Filter jedoch - wir erinnern uns an das Diktum von Michel Serres - würden wir jedes Kopfschütteln unserer Nachbarinnen und Nachbarn hören. Damit sind wir bei der alles entscheidenden Frage der Klangforschung im 19. Jahrhundert: Worin besteht die bedeutungsgenerierende Differenz von Klängen? Und wie realisiert das Ohr die entsprechenden Filter?

Am Leitfaden dieser Grundfrage entwickelten Forscher wie Ernst Chladni, Achim von Arnim, Johann Wilhelm Ritter oder Hans Christian Ørsted einen eigenen Raum der Sichtbarkeit unter einem enormen technischen Aufwand..$^{13}$ Die zentrale experimentelle Strategie der Verwandlung von Klängen in Bilder zum Zwecke ihrer nachgängigen Vermessung, Analyse und Interpretation kennt dabei zwei grundlegende Spielarten: einerseits den Graphen - die in der Fläche operierende Anzeichnung - und andererseits die Glyphe als die im Raum operierende Aufzeichnung. Für den Graphen lassen sich als vielleicht eindrücklichstes Beispiel die manometrischen Flammen von Rudolph Koenig anführen (Abb. 7). >Tönende Luftwellen< werden dabei in sichtbare Schwingungen verwandelt, indem diesseits einer Membran ein brennbares Gas strömt, sodass Schallereignisse jenseits der Membran den Gasstrom geradezu unmittelbar beeinflussen können: ${ }^{14}$ Die Flammen schwingen präzise im Takt der Klänge. Stellt man die Flammen über rotierende Spiegel oder Rußbilder still, so lassen sich beispielsweise Kongruenzen zwischen einzelnen Vokalen und bestimmten Flammenbildern feststellen oder zumindest diskutieren.

Nun können diese Graphen bekanntlich nicht in Klänge zurückverwandelt werden, wohl aber Glyphen, so wie sie beim Grammophon von einer wiederum an einer Membran befestigten Nadel direkt ins Metall geschnitten werden und somit zu einer »écriture naturelle« werden. ${ }^{15}$ Was die Vermutung nahelegt, dass der 3D-Code der Schallplattenrille sehr viel mehr Eigenschaften eines Klangs speichert als die zweidimensionale Spur der Graphen: »[C]et instrument, nul ne l'ignore, c'est le phonographe. ${ }^{16}$ Allerdings zu dem Preis, dass messbare Eigenschaften des

12 Vgl. Eska 1997, 73.

13 Siehe Rieger 2009, 62-73.

14 Koenig 1872, 161.

15 Marichelle 1897, 1 (Hervorh. i. Original).

16 Ebd., 10. 


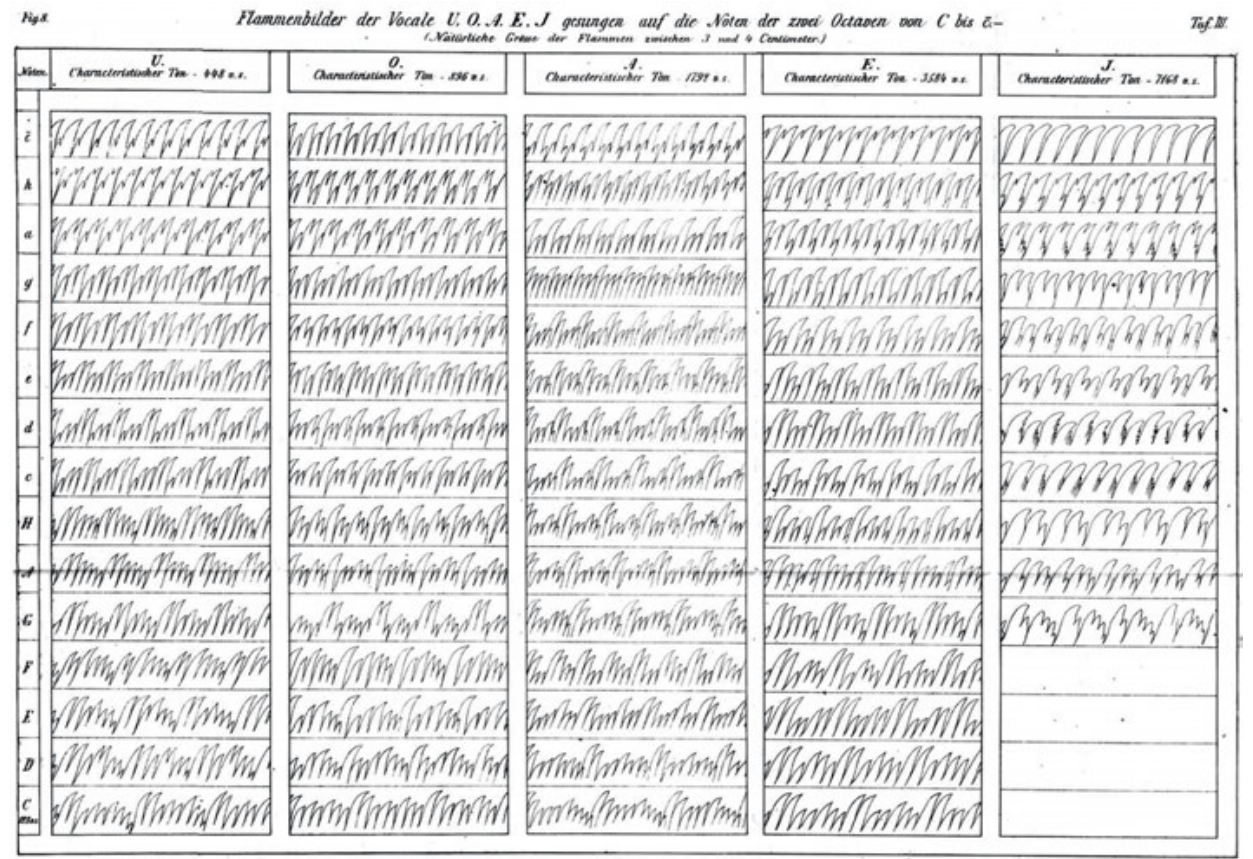

Abb. 7: »Flammenbilder der Vocale «.

Klangs nur aus der Transformation der Glyphen in Graphen gewonnen werden können. Also wurde die Rille mittels Tastnadel in Kurven übersetzt, was jedoch wenig brauchbare Ergebnisse lieferte. Der Phonetiker Giulio Panconcelli-Calzia resümiert in seinem Quellenatlas zur Geschichte der Phonetik: »Daß auf diese Weise nur sehr mangelhafte Ergebnisse zu erzielen waren, steht heute fest; die so erhaltenen Kurven reichen im günstigsten Fall zur Ausmessung der Tonhöhe. $\ll^{17}$ Im weiteren Verlauf wurden Mikroskope, fotografisches Papier, bewegte Spiegel und Ähnliches verwendet, um den Rillen sozusagen auf die Spur zu kommen.

Aus der Masse dieser Medienverbünde möchten wir abschließend ein Verfahren zur Überführung der Glyphen in den Bereich des Sichtbaren genauer beleuchten, weil uns dieses wesentlich für das Verhältnis von Historisierung und Experimentalisierung zu sein scheint. Hector Marichelle, Professor am Pariser Institut für Taubstumme, legte 1897 ein umfängliches Werk zur Parole d'après le tracé du phonographe vor. Zwischen dem Realen der Glyphe und dem Symbolischen des Graphen vermittelte hier nicht erneut ein Apparat, sondern die Hand des Professors selbst. Marichelle hockte über dem Mikroskop und blickte direkt in die Schallspur, um diese mit der freien Hand nachzuzeichnen. So wie das Ohr besser als jedes Phonoskop die Stimme auf der Platte in Vokale und Konsonanten zerlegt, so erkennt die Hand besser als jeder Kymograf die Vokale und Konsonanten in der Rille. Die Umzeichnung Marichelles ist ein epistemischer

17 Panconcelli-Calzia 1940, zit. nach Rieger 2009, 98. 
Prozess, ein Bildakt im weiteren Sinne (Abb. 8). Um dies zu beweisen, stellte er seine Graphen den Flammenbildern gegenüber, was natürlich eine Inszenierung war: Schlägt sich das Reale noch voll in der Form der Flammen nieder, bestechen die phonographischen Figuren durch symbolhafte Eindeutigkeit.

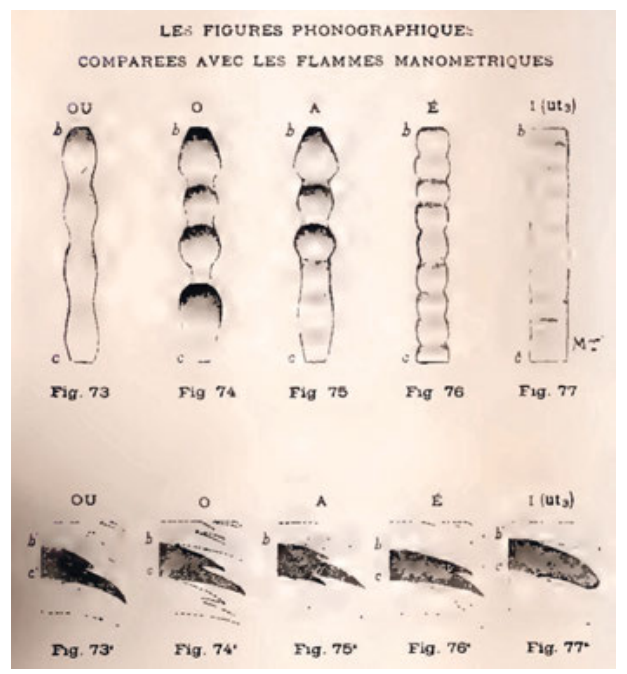

Abb. 8: »Les figures phonographiques comparées avec les flammes manometriques «.

Es ist also eine doppelte Übersetzung, die Marichelle benötigte, um die symbolische Ebene zu erreichen, indem er den sprachlichen Lautstrom der Zylinderrille mit dem Zeichenstift digitalisierte. Marichelle vertraute der Operation des Tastens, das immer zugleich ein Hören der Oberfläche ist, genauso wie die heutigen Experimentalphysikerinnen und -physiker, die mit einem Rasterkraftmikroskop arbeiten. Der interpretatorische Sprung im Prozess der Bildgebung ereignet sich dabei gleichermaßen innerhalb der Wissensgeschichte wie in der aktuellen Forschung. Bilder spielen in der Wissensproduktion der Naturwissenschaften nicht bloß auf der symbolischen Ebene eine entscheidende Rolle. Die Produktion dieser Bilder setzt sehr konkrete Operationen voraus (und das heißt natürlich auch Instrumente und Apparate), die grundsätzlich als epistemische Praktiken zu verstehen und auf ihre bedeutungsgenerierende Funktion hin zu untersuchen sind. 


\section{Literatur}

Binnig, Gerd/Rohrer, Heinrich (1979): Scanning Apparatus for Surface Analysis Using Vacuum-Tunnel Effect at Cryogenic Temperatures (Gerät zur rasterartigen Oberflächenuntersuchung unter Ausnutzung des Vakuum-Tunneleffekts bei kryogenischen Temperaturen). Patent CH643397, 20. September.

Eilers, Stefan (2013): Strukturelle und Elektronische Eigenschaften von Nanographen-Graphen-Systemen sowie Schnitt- und Faltverhalten von Graphen. Diss., Humboldt-Universität zu Berlin. Online unter: http://edoc. hu-berlin.de/dissertationen/eilers-stefan-2013-04-05/ PDF/eilers.pdf (zuletzt aufgerufen: 6. Januar 2015).

Eska, Georg (1997): Schall \& Klang. Wie und was wir hören. Basel/Boston/Berlin: Birkhäuser Verlag.

García-Sánchez, Daniel et al. (2008): Imaging Mechanical Vibrations in Suspended Graphene Sheets. In: Nano Letters, vol. 8, no. 5, pp. 1399-1403.

Hengler, Lorenz (1832): Astronomische Pendelwage, nebst einer neuen Nivellirwage, erfunden und dargestellt von Lorenz Hengler, akademischen Bürger an der Hochschule zu München. In: Polytechnisches Journal, Jg. 43, Nr. 2, S. 81-92.

Kassabian, Anahid (2013): Ubiquitous Listening. Affect, Attention, and Distributed Subjectivity. Berkeley/Los Angeles/London: University of California Press.

Kassung, Christian (2007): Das Pendel. Eine Wissensgeschichte. München: Wilhelm Fink.

Koenig, Rudolph (1872): Die manometrischen Flammen. In: Annalen der Physik und Chemie, Jg.XCLVI, Nr.6, S. $161-199$.
Marichelle, Hector (1897): La parole d'apres le tracé du phonographe. Paris: Librairie Ch. Delagrave.

Panconcelli-Calzia, Giulio (1940): Quellenatlas zur Geschichte der Phonetik. Hamburg: Hansischer Gildenverlag.

Rieger, Stefan (2009): Schall und Rauch. Eine Mediengeschichte der Kurve. Frankfurt a. M.: Suhrkamp.

Schrimshaw, Will (2013): Non-Cochlear Sound: On Affect and Exteriority. In: Biddle, Ian/Thompson, Marie (eds.): Sound, Music, Affect. Theorizing Sonic Experience. New York/London: Bloomsbury, pp. 27-44.

Serres, Michel (1987): Der Parasit. Frankfurt a. M.: Suhrkamp.

Zöllner, Johann Karl Friedrich (1869): Ueber eine neue Methode zur Messung anziehender und abstossender Kräfte. In: Berichte über die Verhandlungen der Königlich Sächsischen Gesellschaft der Wissenschaften zu Leipzig. Mathematisch-Physische Classe, Nr. 21, S. 281-284.

Zöllner, Johann Karl Friedrich (1871): Ueber den Ursprung des Erdmagnetismus und die magnetischen Beziehungen der Körper. In: Berichte über die Verhandlungen der Königlich Sächsischen Gesellschaft der Wissenschaften zu Leipzig. Mathematisch-Physische Classe, Nr. 23, S. 479-575.

Zöllner, Johann Karl Friedrich (1873): Zur Geschichte des Horizontalpendels. In: Annalen der Physik, vol. 226, no. 9, pp.140-150. Online: http://onlinelibrary.wiley. com/doi/10.1002/andp.18732260909/epdf (last access: 29 May 2015). 


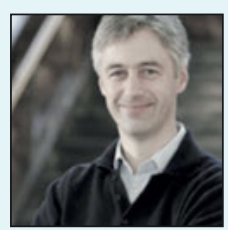

ckassung@culture.hu-berlin.de

Principal Investigator

Basisprojekte: Analogspeicher, Piktogramme

Disziplin: Kulturwissenschaft

Christian Kassung ist seit 2006 Professor für Kulturtechniken und Wissensgeschichte an der Humboldt-Universität zu Berlin. Kassung ist Vizedirektor des Hermann von Helmholtz-Zentrums für Kulturtechnik. Seinen Forschungsschwerpunkten der Wissens- und Kulturgeschichte der Naturwissenschaften, vor allem der Physik, der Geschichte und Praxis technischer Medien sowie der Literaturund Kulturtheorie spürt er am Exzellenzcluster Bild Wissen Gestaltung in zahlreichen Positionen und Projekten nach. 


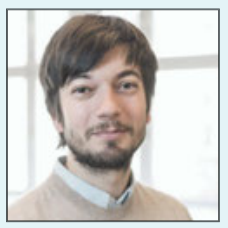

sebastian.schwesinger@hu-berlin.de

Wissenschaftlicher Mitarbeiter

Basisprojekt: Analogspeicher

Disziplin: Kulturwissenschaft, Sound Studies

Sebastian Schwesinger studierte International Management und Controlling an der Hochschule für Ökonomie \& Management und der Hogeschool Zeeland, anschließend Kulturwissenschaft, Musikwissenschaft und Philosophie an der Humboldt-Universität zu Berlin und der London Metropolitan University. Am Exzellenzcluster Bild Wissen Gestaltung forscht er zum Verhältnis von Klang, Materialität und Infrastrukturdesign. Daneben leitet er ein Lehr- und Forschungsprojekt zur Auditiven Kulturwissenschaft, produziert künstlerisch-wissenschaftliche Hörstücke, ist Gründer des experimentellen Medienprojekts soniK RadiofabriK und Mitglied des gamelab.berlin.

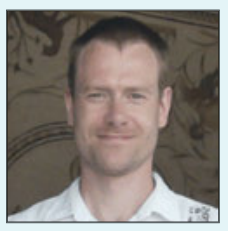

christian.seifert@physik.hu-berlin.de

Wissenschaftlicher Mitarbeiter

Basisprojekt: Analogspeicher

Disziplin: Physik

Christian Seifert machte einen Abschluss als Kommunikationselektroniker bei Siemens und studierte im Anschluss Physikalische Technik mit Fachrichtung Medizinphysik und Physik an der Technischen Fachhochschule Berlin und der Humboldt-Universität zu Berlin. Seit 2009 arbeitet er an letzterer als wissenschaftlicher Mitarbeiter und erforscht im Rahmen seiner Promotion, wie sich kleinste elektrische Ströme und Einzelmoleküle kontrollieren lassen. 


\section{Bilder der Bewegung}

\section{Sexualität im Zellkern um 1900 und nach 2000}

In welch komplexer Art auch immer die Instrumente der Biowissenschaften gestaltet sein mögen, sie alle dienen primär dazu, unseren Sinnen Phänomene zugänglich zu machen, die sonst außerhalb unserer Wahrnehmung lägen.' In den meisten Fällen wird der visuelle Sinn bevorzugt, dem etwa das Mikroskop zuspielt. ${ }^{2}$ Davon werden heutige Methoden der digitalen Bildgebung meist scharf unterschieden - nach Schröter wurde die Trennung von analog und digital zur medientheoretischen Leitdifferenz der vergangenen Jahrzehnte. ${ }^{3}$

Die vorliegende Analyse gilt einem fragmentarischen Vergleich der visuellen Laborkultur des späten 19. Jahrhunderts mit jener der Zeit nach 2000 und stellt dabei eine solche Differenz in Frage. Zwar mag eine vergleichende Analyse über den Zeitraum eines Jahrhunderts hinweg problematisch erscheinen, doch die Gegenüberstellung dieser zwei Forschungskulturen zeigt mehrere gemeinsame Fragestellungen auf, wie im Folgenden ausgeführt wird. Darüber hinaus behandelt der Vergleich zwei Momente der Änderung in der Technik der Bildgebung (oder des Verzichts auf sie), die miteinander in einen epistemologischen und instrumentengeschichtlichen Zusammenhang gestellt werden können. Der Zusammenhang bezieht sich auf das Verhältnis 
von Bildlichkeit, Zeit und Raum in der biologischen Zelle im Feld der an Vererbung interessierten Zeugungsforschung: In den 1870er-Jahren machten sich in der Forschungsmethodologie zu Befruchtung erste Abstrahierungen bemerkbar. Eine Verschiebung weg von der als Beobachtung des > real< lebenden Objekts propagierten Methode hin zur Produktion von eingefärbten und fixierten Objekten, die dann in ihrem zeitlichen Ablauf angeordnet werden können. Hiervon handelt der folgende zweite Abschnitt dieses Beitrags, ausgeführt am Beispiel der von Oscar Hertwig 1876 veröffentlichten Studie zur Befruchtung. Er zeigt den Übergang von der Lebendbeobachtung zur Anfertigung von Proben, die von der organismischen Zeit unabhängig sind, in einer einzigen Studie. Im Gegensatz zu den meisten zwischen 1870 und 1900 durchgeführten mikroskopischen Studien über die Verschmelzung von Spermien- und Eizelle, der Verschmelzung der Nuklei oder der weiteren Entwicklung der Zelle ragt Hertwigs Studie als eine der ersten heraus, in denen die systematische Einfärbung und Fixierung von Objekten und die Erstellung einer Abfolge von Bildern angewandt, en détail diskutiert und zudem zur Lebendbeobachtung ins Verhältnis gesetzt wurde.

Dieser im Verlauf dann zunehmende Abstraktionsprozess unterstützt ein methodisches Beiseitelassen räumlich-zeitlicher Beobachtung, was schließlich im Moment der Herausbildung der Genetik insofern zum Programm gemacht wird, als die >Geschichtlichkeit< der Lebewesen zugunsten eines an Adolphe Quetelet anschließenden quasi-strukturalistischen Programms der Kopplung von Genotyp und Phänotyp aus dem Fokus gerückt wird. ${ }^{4}$

Dies wird nach 2000 als Problem gesehen und in der inzwischen epigenetisch beeinflussten Reproduktionsgenetik angesprochen, wie im dritten Abschnitt genauer behandelt.

Der Beitrag beruht auf einer Analyse von Studien zu Befruchtung - einerseits aus der >Vorphase der Genetik< zwischen 1870 und 1910 und andererseits solcher der Reproduktionsgenetik, die zwischen 1997 und 2011 veröffentlicht wurden - sowie einer Auswahl von Interviews mit führenden Forschungstätigen auf diesem Gebiet. ${ }^{5}$

Der Vergleich der frühen mikroskopischen Reproduktionsforschung Ende des 19. Jahrhunderts mit den Praktiken in den Jahren vor und nach 2000 zeigt, dass zeitlich-räumliche Phänomene, die seit der Verschiebung nach 1900 lange aus der Genetik ausgeschlossen waren, in den Jahren nach 2000 wieder aufscheinen und eine Vergleichbarkeit der Bildgebung mit sich bringen.

4 Vgl. Johannsen 1911; Kogge 2012; Rheinberger/Müller-Wille 2009.

5 Der Bereich der Reproduktionsgenetik wurde seit 1990 zunehmend durch entsprechend benannte Professuren und Publikationen mit dem Begriff im Titel als Forschungsfeld sichtbar. 


\section{Von der Norm der Lebendzeichnung zum >Still-Leben< auf dem Objektträger}

Intensiv widmete sich die wissenschaftliche Mikroskopie des 19. Jahrhunderts der Schulung des Blicks, der Handhabung des Instruments und der Perfektion des Zeichnens ${ }^{6}$ des im besten Fall

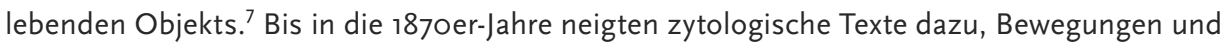
Strukturen in lebhaften und bildreichen Worten zu beschreiben, welche die Lebendigkeit des Prozesses heraufbeschworen. Doch in späteren Jahren wurden diese wortreichen Beschreibungen zunehmend durch Kupferdrucke ersetzt. In den Publikationen der Reproduktionsforschung im späten 19. Jahrhundert wurde versichert, dass die Bilder aussagekräftiger seien als der reine Text und vertrauenswürdiger als bloße Worte. ${ }^{8}$ Die Vorreiterstellung des Visuellen mit den im Zuge dieser Argumentation entstandenen Repräsentationen der Forschungsergebnisse fand zunehmend den Weg in die wissenschaftlichen Publikationen, wie wir sie heute mit ihren vielen Abbildungen als üblich wahrnehmen.

Der Zoologe Oscar Hertwig, der bei Ernst Haeckel in Jena und bei dem Mikroskopiker Max Schultze in Bonn studiert hatte, nutzte diesen Trend zur Überzeugung seines Publikums auf sachliche Weise, und das in einem umstrittenen Feld: Seit den 186oer-Jahren wurde durch Studien bestätigt, dass bei der Befruchtung Spermien- und Eizelle miteinander verschmelzen, und auch Hertwig konnte mit einem Bericht dazu beitragen. ${ }^{9}$ Weiterhin unklar blieb aber die Frage, was mit den Nuklei während und nach der Befruchtung passierte, deren Auflösung der Pathologe Leopold Auerbach an einem lebenden Objekt beobachtet haben wollte. ${ }^{10}$ Zerfielen also die Nuklei womöglich direkt nach der Befruchtung oder verschmolzen sie und blieb dieser Nukleus im neuen Embryo bestehen? In seinem 1876 veröffentlichten detaillierten Bericht Beiträge zur Erkenntnis der Bildung, Befruchtung und Theilung des thierischen Eies verfolgt und beschreibt Hertwig die Bewegungen der Nuklei durch den gesamten Kernzyklus hindurch." Seine Beobachtungen hierfür stellte er am Ozeanografischen Institut Villefrance-sur-Mer bei Nizza an Seeigeln an, um mittels direktem Zugang zu zirkulierendem Meerwasser die nötigen Lebensbedingungen für die Untersuchung der transparenten Meerestiere herzustellen. In der auch praktisch absichtlich leicht nachvollziehbaren Beschreibung seiner Beobachtung »lebendiger Objekte $\ll^{12}$ unter dem Zeiss-Mikroskop dient Hertwig die Beobachtung der Bewegung

6 Siehe Chadarevian 1994; Daston/Galison 2007, 17-51; vgl. Schickore 2007; Clarke/Jacyna 1987; Schleiden 1842/43, 120ff.; Gooday 1991; Wahrig-Schmidt 1994. Zu den Diskussionen über die korrekte Anwendung des Mikroskops in Bezug auf Befruchtung siehe z. B. Bischoff 1854 .

7 Siehe Schmidgen 2004 .

8 Siehe z. B. Boveri 1889, 77; Kölliker 1895, 7.

9 Siehe Hertwig 1876. Die Wissenschaftsgeschichte zitiert meist Hermann Fols 2 Jahre später erfolgte Veröffentlichung zur Erkenntnis über das Eindringen des Spermiums (Fol 1877; siehe z. B. Cremer/Cremer 2009), obwohl zeitgenössische Besprechungen Ende des 19. und zu Beginn des 20. Jahrhunderts Hertwigs überzeugende Berichte zur Befruchtung lobten.

10 Siehe Auerbach 1874 .

11 Hertwig 1876.

12 Ebd., 380. 

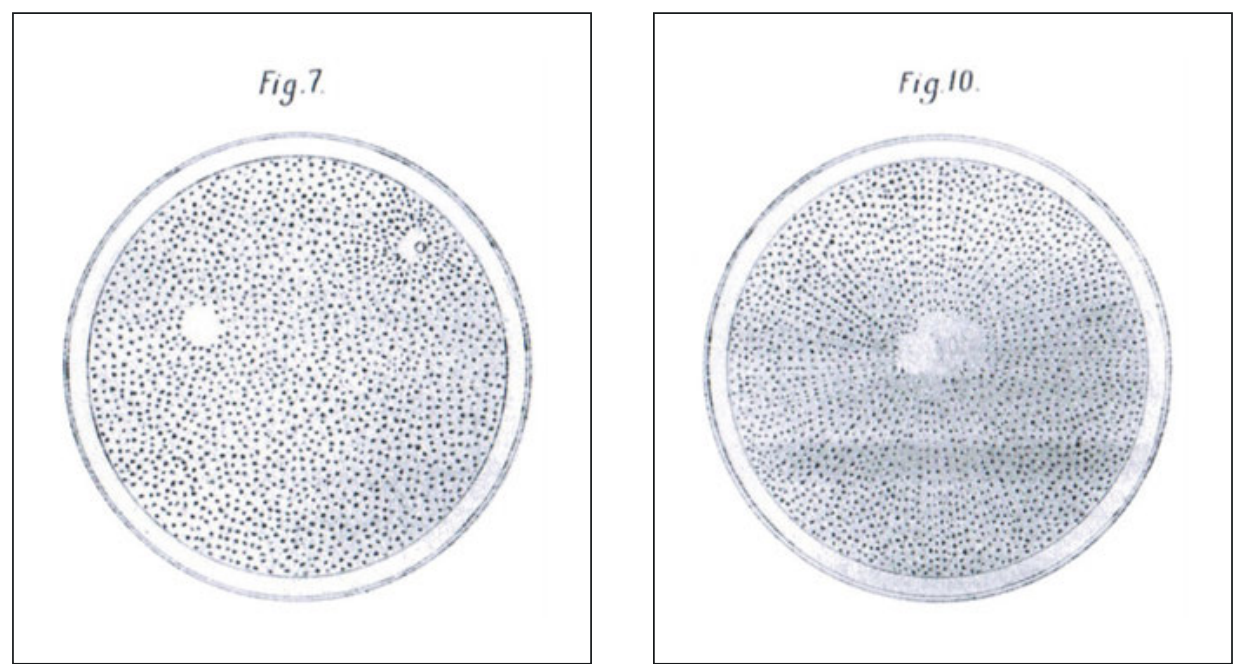

Abb. 1: Drucke der Ansichten Hertwigs durch sein Zeiss-Mikroskop, 5 (Fig. 7) bzw. 10 (Fig. 10) min. nach der Befruchtung eines Seeigeleis.

als Beweis dafür, dass der Nukleus des Spermiums mit dem der Eizelle verschmilzt. Hertwig betont auch, dass er seine Beobachtungen überprüft habe: »Dass aber eine Bewegung stattfindet, davon habe ich mich ganz sicher überzeugt, indem ich die Lageveränderung des Kerns mit dem Mikrometer controlirte. $\ll^{13}$ Die Bilder dieser $\gg$ Lebenserscheinungen $\ll^{14}$ innerhalb der Zelle mussten nun gezeichnet werden, während man dem Objekt in Bewegung folgte. Dies führte er in fünfminütigen Intervallen durch (Abb. 1), sodass der Text zu seinen Bildern 7 bis 11, die dem mehrere 100 Seiten umfassenden Artikel als Tafeln angehängt sind, wie folgt lautet:

»Figur 7 und 8. Ei, fünf Minuten nach der Befruchtung. Einwandern des Spermakerns. $[\ldots]$

Figur 10. Ei, zehn Minuten nach der Befruchtung. Ei- und Spermakern berühren sich.

Figur 11. Ei, eine Viertelstunde nach der Befruchtung, mit dem durch Verschmelzung des Ei- und Spermakerns entstandenen Furchungskern. $\ll^{15}$

Hertwigs Studie hatte darauf abgezielt, zu zeigen, was genau mit den Zellnuklei auch nach der Befruchtung geschieht. Doch aufgrund der geringen Auflösung des Mikroskops entging dies seinen Lebendbeobachtungen. Im Bewusstsein dieser Einschränkung machte Hertwig anläss-

13 Hertwig 1876, 380.

14 Ebd., 349.

15 Hertwig 1876, Anhang, Beschreibung zu Taf. 11. 
lich der ersten sommerlichen Gelegenheit am französischen Meer von den unterschiedlichen chemischen Färbemethoden Gebrauch, die 1873 erstmals in einer Veröffentlichung methodisch propagiert worden waren. ${ }^{16}$

Das Einfärben der Zelle bedeutete, dass diese getötet werden musste. Dementsprechend schuf Hertwig unterschiedliche fixierte Stadien, die er dann in einer Reihe von Bildern zellulärer Momente zusammenstellte. Die Bewegung der Zellen stoppte Hertwig nach standardisierten Zeitabständen (wiederum 5 min., 10 min. etc. nach der Befruchtung, das heißt, nachdem die Keimzellen des Seeigels in einer Flasche zusammengeschüttelt worden waren). Begleitet wurde diese praktische Arbeit an den Zellen von der sich verbreitenden Darstellungsweise, wonach es sich hierbei um spezifische Stadien, um $»$ Schritte $\ll,{ }^{17}$ handele. Es sei daher nötig, »[z]um Zweck der Untersuchung [...] die künstliche Befruchtung einzuleiten, um den Zeitpunct, wo Sperma und Eier sich treffen, genau bestimmen zu können «. ${ }^{8}$ Ziel war es, auf diese Weise »die Entwicklung einzuleiten und dieselbe von Anfang bis Ende Schritt für Schritt zu verfolgen «. ${ }^{19}$ Die Bedeutung dieses Vorgehens betont Hertwig nochmals in einer Kollegenschelte gegen seine Vorgänger Karl von Baer, Eduard Strasburger, Franz Leydig und andere, da keiner von ihnen »die Theilungsvorgänge in allen Stadien Schritt für Schritt genau verfolgt und beschrieben « habe, ${ }^{20}$ oder sie hätten, wie Auerbach, »die Möglichkeit, dass der Kern im frischen Zustande unkenntlich geworden ist, nicht berücksichtigt «.21

Analog zur neuen Färbetechnik, die es unmöglich machte, Bewegung in lebenden Zellen zu verfolgen, wurden die Zustände in der Zelle in zellbiologischen Texten zu Befruchtung und Vererbung während der letzten 20 Jahre des 19. Jahrhunderts zunehmend als statisch beschrieben. Die Formierungen von Chromosomen (gr. chroma [Farbe] und soma [Körper], wie eingeführt von Waldeyer ${ }^{22}$ wurden ebenfalls verbal als in bestimmten Phasen der Mitose aufeinanderfolgende Figuren bezeichnet (Abb. 2).

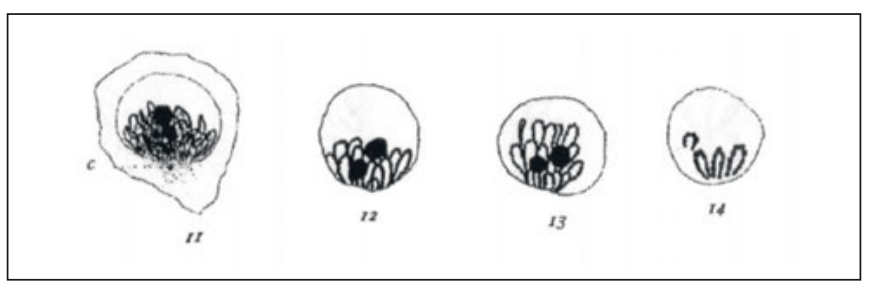

Abb. 2: Bouquetphase aus den Studies in Spermatogenesis von Nettie Maria Stevens, 1905 .

\footnotetext{
16 Durch Abbé 1873.

17 Hertwig 1876, 376 ff.

18 Ebd., 378.

19 Ebd., 398.

20 Ebd., 419, Hervorh. BvW.

21 Hertwig 1876, 425, Hervorh. BvW.

22 Siehe Waldeyer 1888.
} 
Die bald standardisierten Formationen wurden mit den noch heute verwendeten Begriffen Bouquetphase, Kreuz, Diamant, Hantelphase etc. bezeichnet und geprägt durch Chromosomenforschungen wie die Dissertation von Nettie Maria Stevens zu ihrer Entdeckung des >männlichen< Chromosoms. ${ }^{23}$ Stevens' für die Forschung zu dieser Zeit typischer Text besteht bereits hauptsächlich aus einer Reihe von Titeln zu Bildern, die die unterschiedlichen Phasen zeitlich anordnen; der Anhang zu ihrer Dissertation besteht aus 241 Zeichnungen, während der 30-seitige Text beschreibt, was wir auf ihnen sehen können (oder sehen sollten).

Die visuelle Ikonografie ${ }^{24}$ der eingefärbten Materialien besteht aus einer Rekomposition der statischen Elemente in eine standardisierte nicht prozedurale, deterministische und unidirektionale Abfolge von Zuständen. Laut Griesemer war das zeitliche Verständnis der embryonalen Zytologie direkt mit dem der Genetik des 20. Jahrhunderts verbunden. ${ }^{25}$

Zeit wurde in diesem Zusammenhang visuell noch in der Differenz in räumlicher Struktur dargestellt, ${ }^{26}$ obwohl die embryologischen Darstellungen an sich statisch und abstrakt waren. Die visuellen Abstraktionen stellten einen historischen Übergang dar, denn sie kreierten »two visual abstractions which were important to the emergence, in the $20^{\text {th }}$ century, of distinct narrative forms in transmission genetics and developmental biology: the genealogical time of the geneticist and the deterministic temporal order of the developmental biologist «. ${ }^{27}$

Schließlich gerieten in einem weiteren Abstraktionsprozess im Zuge der Aneinanderrückung von Genotyp und Phänotyp im neuen Programm der klassischen Genetik zwei Phänomene aus dem Fokus: die Betrachtung von Veränderung im Raum und zugleich, so Rheinberger und Müller-Wille, die Geschichte im Sinne der Onto- und Phylogenese. ${ }^{28}$

Dies änderte sich in den 1990er-Jahren sichtbar mit Forschungsprogrammen, die, etwa unter epigenetischen Aspekten, die räumliche Zelle im wörtlichen Sinne wieder stärker in den Blick nahmen. ${ }^{29}$

\section{Reproduktionsgenetik: Befruchtung und Vererbung im Trockenlabor}

Angestoßen vor allem durch das Humangenomprojekt sowie durch die anschließende Kritik, dass dieses zwar Daten, aber keine Information über deren Bedeutungen geliefert habe, hat sich die Ausrichtung der Zeugungs- und Vererbungsforschung deutlich verändert. Seit den 1990er-Jahren haben sich neue Forschungsgebiete in der Genetik entwickelt, von denen sich

23 Siehe Stevens 1905.

24 Siehe Wellmann 2011; Hopwood/Schaffer/Secord 2010.

25 Siehe Griesemer 2002.

26 Ebd., 50.

27 Griesemer 2002, 46.

28 Siehe Rheinberger/Müller-Wille 2009.

29 Siehe Franklin 2007; Landecker 2005; dies. 2007; Nowotny/Testa 2010. 
manche mit räumlichen Fragen auseinandersetzen. Bioinformatik hat dabei eine entscheidende Rolle gespielt.

Bislang mangelte es den Forschenden in der Reproduktionsgenetik an Möglichkeiten, die großen Datenmengen, die sie sammelten, auch zu bewältigen. Seit Mitte der 20ooer-Jahre und mit der Beschleunigung der genetischen Analyse wie im Microarray ${ }^{30}$ wird jedoch die Visualisierung für die (Epi-)Genetik und -Genomik relevanter Datenmengen selbst in zeitlicher Abfolge und im räumlichen Vergleich (zwischen verschiedenen Zellen oder gar Geweben) möglich.

Zusätzlich zum üblichen Tiermodell sind nun auch Computersimulationen in das Biolabor eingezogen, die einen Großteil der Arbeit in den früheren Nasslaboren ersetzen. ${ }^{31}$ Die Arbeit im Labor der Computer und Mikroroboter, also im sogenannten Trockenlabor, ermöglicht Material- und Zeitersparnis in den Forschungsansatz veränderndem Ausmaß. Dies lässt sich auch in der bildlichen Epistemologie der Studien der vergangenen zehn Jahre ablesen. Bis etwa 2005 überwogen in der Reproduktionsgenetik Studien, die analog zur frühen klassischen Genetik mit der direkten Kopplung eines genetischen Phänomens mit einem phänotypischen Befund arbeiteten, basierend auf der Technik des Haplotyping, ähnlich einer Genkartierung. ${ }^{32}$ Die zeitliche Anordnung ist hier ein Vergleich zwischen zwei Gegenwarten; es gibt keine räumliche oder zeitliche Perspektive. Der direkte Zusammenhang zwischen dem Gen und seinem phänotypischen Ausdruck wird noch durch ihre parallele Symbolisierung unterstrichen: Entsprechend der Standardbezeichnungen wird das > REC8 Gen < direkt in das > Rec8 Protein < übersetzt. ${ }^{33}$ Umweltbedingte und genetische Faktoren werden hier als konkurrierende und nicht potenziell interagierende Modelle betrachtet. ${ }^{34}$ Die Bildgebung besteht überwiegend aus Tabellen der Gegenüberstellung von Genbezeichnungen und korrespondierenden Effekten.

Inzwischen mehren sich Studien, die auf Methoden basieren, welche komplexere Beziehungen zwischen Ursache und Wirkung voraussetzen, etwa Betrachtungen der Hormonwirkungen auf die DNA, ${ }^{35}$ die häufig als Pfeildiagramme dargestellt werden. ${ }^{36}$ Andere Studien verwenden die Transkriptomanalyse, ${ }^{37}$ welche auf der Annahme basiert, dass Zellen zwar die gleiche genetische Ausstattung haben können, die Verwendung dieser Gene jedoch ausschlaggebend sein und zwischen unterschiedlichen Zellen und zu unterschiedlichen Zeiten sehr divers ausfallen kann. ${ }^{38}$ Studien dieser Art untersuchen den Einfluss des spezifischen lokalen Umfelds einer Zelle auf die DNA und ihre Transkripte, was mit Haplotyping nicht zu erreichen wäre. Inzwischen haben die Transkriptomanalyse und Real-time-PCR (Polymerase Chain Reaction) das Haplotyping

30 Bei dieser Methode werden winzige DNA-Proben gleichzeitig mit fluoreszierenden Substanzen markiert, die dann von einem Computerprogramm ausgelesen werden.

31 Siehe Morgan 2003; dies. 2005; Merz 2006.

32 Siehe Lin et al. 2005; Coulam et al. 2007, 40.

33 Siehe Furnes/Schimenti 2007, 29.

34 Ebd., 30.

35 Siehe Drummond 2006; Schaffner 2007.

36 Drummond 2006.

37 Z. B. Jelinski et al. 2007; Shima et al. 2004.

38 Siehe Liu et al. 2011; Wang et al. 2011. 
verdrängt. Mit der Echtzeit-PCR wird gemessen, wie viel von den jeweiligen DNA-Abschnitten in den jeweiligen Zellen vorliegt. Dies wird als Maß dafür verstanden, wie häufig in dem Moment bestimmte Genabschnitte aktiviert worden sind. Auch Forschungen zu Genomic Imprinting - also zur epigenetischen Prägung - finden sich zunehmend. Hier wird die Fähigkeit ausgewachsener Zellen untersucht, Genome zu »reprogrammieren $\ll{ }^{39}$ Reprogrammierung meint, dass ein Genom, das epigenetisch auf eine bestimmte Arbeitsfunktion in den frühen Stadien eines Embryos festgelegt war, später noch zu einer völlig anderen Funktion hin verändert werden kann. Dies wird auch als Zeitumkehrung bezeichnet. ${ }^{40}$ Die Ergebnisse präsentieren parallel ablaufende Prozesse von verschiedenen Geschwindigkeiten. Systembiolog_innen betonen, dass es nur mit der computerbasierten Berechnung hoher Datenmengen möglich sei, Modelle zu erstellen, die derart integrativ und komplex sind, dass sie Dimensionen von Zeit und Raum erfassen. ${ }^{41}$ Dem entspricht auf genetischer Seite der bereits genannte in Roboter und Computer integrierte Microarray. Wie eine Reproduktionsgenetikerin im Interview erklärte, sei dieser im Gegensatz zu älteren Techniken sogar in der Lage, einen » molekularen Karyotyp « zu erstellen. ${ }^{42}$

Für sie bedeute der Blick auf den Ergebnisausdruck (Abb.3):

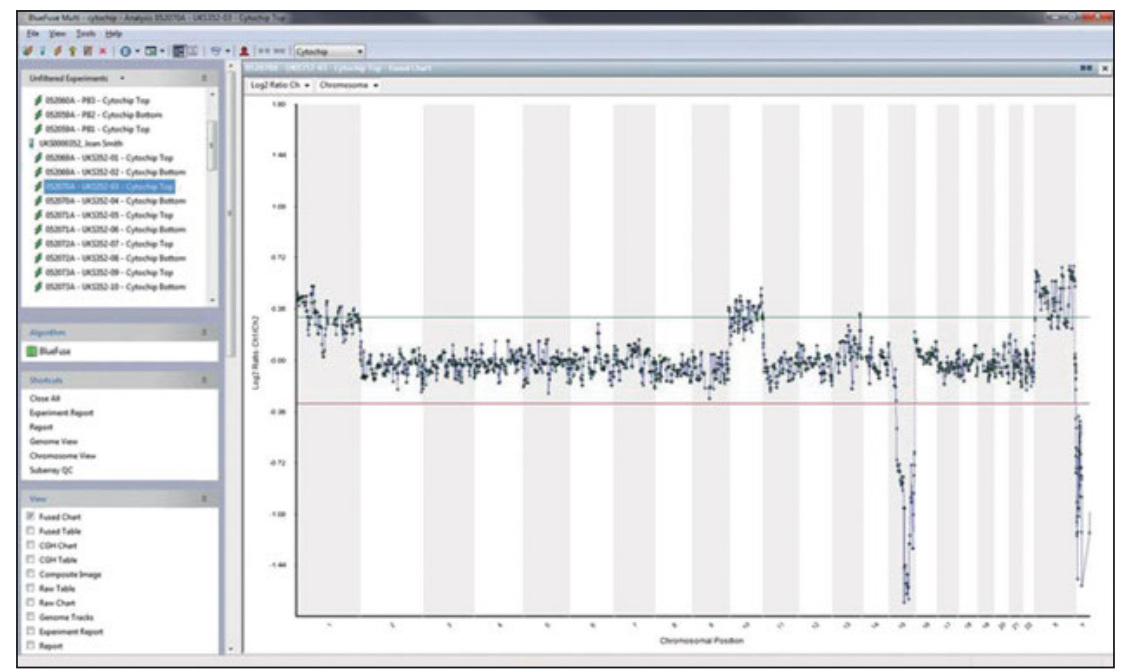

Abb. 3: Bild eines Chromosomen per Microarray.

»You can look at the chromosomes [...]. [Now] you can see all the chromosomes even in the single cell, [...] you know of every 23 chromosomes, are there too many, are they too little, [...]. And it gives you much higher resolution. We want to go to the

39 Strelchenko et al. 2006; siehe auch Wilkins-Haug 2009.

40 Siehe Nowotny/Testa 2010.

41 Siehe z. B. Kitani 2001.

42 Sermon 2011. 
fundamental biology of [...] chromosomes in embryos - we just needed this kind of precise instrument to look at it. «3

In ihrer Wortwahl fallen Begriffe auf, die aus der Lichtmikroskopie vertraut sind, wie etwa »Auflösung « (»resolution «). Tatsächlich ist der Unterschied zwischen dem Lichtmikroskop und der Datenverarbeitung des Micorarray im bildgebenden Computer für die Wissenschaftlerin unwesentlich: Was wir heute im Computerausdruck des Microarray sähen, sei genauso wie das Bild im Mikroskop »fact«-manipulierte Darstellung. Wir sähen genauso Chromosomen, »just different representations « im Verhältnis zu jenen, die im Lichtmikroskop zu sehen seien. ${ }^{44}$ In der Tat liefert bereits das schlichteste historische Lichtmikroskop Bilder, die mit jenen, die dem Auge ohne technische Hilfe erscheinen, nicht vergleichbar sind.$^{45}$ Denn die Physik der mikroskopischen Optik ist eine von der des üblichen Sehens ohne Instrument gänzlich verschiedene: nämlich die der Diffraktion statt der Refraktion. Färbung und Emersion fügen lediglich weitere Unterschiede hinzu. Ausgehend von Hackings praxeologischem Ansatz macht es dennoch Sinn, davon zu sprechen, wir würden etwas > sehen < durch die verschiedenen Instrumente. Wir >sehen< durch das Lichtmikroskop, wenn »[the] image is a map of interaction between the specimen and the image of radiation and the map is a good one $\ll .{ }^{46}$

\section{Bilder der Zeugungsvorgänge im Kern in den 1870er-Jahren und nach 2000}

Im Vergleich zu Zeichnungen stellt eine Reihe von eingefärbten Objekten eine materielle Beweisführung des Zeitverlaufs dar, was für die »Semiotik von Zeit « ${ }^{47}$ in Experimenten ausschlaggebend ist. Auch wenn es für ihre Abzeichnung noch immer Auge und Hand des Forschers oder der Forscherin bedarf, ${ }^{48}$ so scheinen gefärbte und fixierte Objekte im Sinne einer mechanischen Objektivität für sich selbst zu sprechen. ${ }^{49}$ In der Technik von Einfärben und Fixieren wird die Zeit selbst zum Instrument: Hier dient das Anhalten der Zeit der Verbesserung der räumlichen Sichtbarkeit und der Gewinnung einer materiellen Nachweisbarkeit der Unterschiede zwischen den Objekten, die sich auf den Objektträgern zeigen. Die Träger werden so, ähnlich Fotos oder Tonträgern, zu >Zeitspeichern $<.{ }^{50}$ Ähnlich der Fotografie, die kurze Zeit später zur Verwendung kam,$^{51}$ oder dem Film konservieren sie einen sechten < Zustand durch technische Mittel. Indem die fixierten Objekte ohne die Einwirkung von Subjekten zu funktionieren scheinen, vermitteln

\footnotetext{
43 Ebd., Hervorh. i. Orig.

44 Ebd.

45 Siehe Hacking 1985.

46 Ebd., 151.

47 Schmidgen 2007, 61.

48 Siehe Latour 1986; Bredekamp 2005.

49 Siehe Daston/Galison 2007.

50 Siehe Doane 2005; Kittler 1986.

51 Z. B. bei Wilson 1895 .
} 
sie einen besonders starken Eindruck von Objektivität. ${ }^{52}$ Die mikrokospischen Färbepräparate geben allerdings bereits einen Vorgeschmack auf die Experimentalsysteme der Reproduktionsgenetik von heute, indem sie für ein bestimmtes Instrument zugerichtet sind und ausschließlich mit diesem gemeinsam - sowie mit dem entsprechenden mikroskopisch trainierten Personal - ein sinnerschließendes Bild ergeben.

Den heutigen Methoden allerdings wird in hohem Maße Manipulation unterstellt, während die Mikroskopie in einer Verlängerung des Auges tiefere Schichten der Natur bloßzulegen scheint. ${ }^{53}$ Indem die Zurichtung der Probe auf dem Objektträger deutlich wird, relativiert sich diese Differenz, ohne dass eine direkte Kontinuität behauptet werden muss. Der Microarray und auf diesem basierende Computermodellierungen von Zeitabläufen der Genaktivität im Zeugungsprozess schließen - in digitaler Form - an frühere mechanische Objektivität an: die Bilder sind nicht allein errechnet, ${ }^{54}$ sondern stehen in proportionalem Zusammenhang mit der Materialität der Proben. Entsprechend betont die Techniktheoretikerin Martina Heßler: »Im Hinblick auf die Referenz unterscheiden sich diese digitalisierten Bilder nicht von analog hergestellten Bildern des 19. Jahrhunderts, die auf Messreihen beruhen wie die Messungen des Pflanzenwachstums, des Pulses, von Temperaturen etc. «. ${ }^{55}$ Indem die Proben sich über ihre materiellen Eigenschaften ansichtig machen, hinterlassen sie eine $» S p u r \ll .{ }^{56}$ Die jeweiligen Kurven werden gemessen an ebenfalls aus der Zeit genommenen Proben (ihre jeweilige Zeit steht also still, während jene des Versuchsobjekts - vermutlich - weiterläuft). Aus biologischer Sicht gibt der Microarray ein Bild auf der Ebene des Molekularen, also in einer Auflösung, welche die optische Physik des Mikroskops nicht erreicht. Im Unterschied zu vorherigen genetischen Methoden steigt damit zugleich das Interesse an räumlich-zeitlichen Kategorien und an erhöhter Komplexität - Aspekte, für deren überwiegende Abwesenheit die Genetik oft kritisiert wurde. Entscheidend für die Bildkritik heutiger Methoden ist demnach nicht allein, dass (in hohem Masse) Daten verrechnet werden, sondern in welchem Maße das resultierende Bild eine »gute $\ll^{57}$ Karte ergibt. Dafür sind in allen Instrumenten der Bildgebung ebenso wie in einem letztlich auch immer »social microscope ${ }^{58}$ nicht zuletzt der gewählte Ausschnitt und die Auswahl der Kategorien für die Legende entscheidend.

52 Siehe Rheinberger 2003.

53 Siehe Hacking 1985.

$54 \mathrm{Vgl}$. die Unterscheidung von algorithmischen und »abgetasteten« Bildern, Schröter 2004, 337.

55 Heßler 2006, Hervorh. BvW.

56 Ginzburg 1980.

57 Hacking 1985, 151.

58 Allchin 1999. 


\section{Literatur}

Allchin, Douglas (1999): Do We See through a Social Microscope? Credibility As a Vicarious Selector. In: Philosophy of Science no. 60 , pp. $287-298$.

Auerbach, Leopold (1874): Organologische Studien 1: Zur Charakteristik und Lebensgeschichte der Zellkerne. Breslau: Morgenstern.

Bischoff, Theodor (1854): Widerlegung der von Dr. Keber bei den Najaden und Dr. Nelson bei den Ascariden behaupteten Eindringens der Spermatozoiden in das Ei. Gießen: J. Ricker'sche Buchhandlung.

BlueGnome (2011): 24sure ${ }^{T M}$. Information brochure. Cambridge: Head Office, BlueGnome.

Boveri, Theodor (1889): Ein geschlechtlich erzeugter Organismus ohne mütterliche Eigenschaften. In: Sitzungsberichte der Gesellschaft für Morphologie und Physiologie in München, Bd. 5 , S. $73-80$.

Bredekamp, Horst (2005): Darwins Korallen: Frühe Evolutions modelle und die Tradition der Naturgeschichte. Berlin: Wagenbach.

Chadarevian, Soraya de (1994): Sehen und Aufzeichnen in der Botanik des 19. Jahrhunderts. In: Wetzel, Michael/Wolf, Herta (Hg.): Der Entzug der Bilder. Visuelle Realitäten. München: Wil helm Fink, S. 121-144.

Clarke, Edwin/Jacyna, Steven L. (1987): Nineteenth-Century Origins of Neuroscientific Concepts. Berkeley: University of California Press.

Coulam, Carolyn B. et al. (2007): Discordance among Blastomeres Renders Preimplantation Genetic Diagnosis for Aneuploidy Ineffective. In: Journal of Assisted Reproduction and Genetics no. 24, pp. $37-41$.

Cremer, Thomas/Cremer, Christoph (2009): Rise, Fall and $\boldsymbol{R} \boldsymbol{e}$ surrection of Chromosome Territories: a Historical Perspective. Part I. The Rise of Chromosome Territories. In: European Journal of Histochemistry, vol.50, no. 3, pp. 161-176.

Daston, Lorraine/Galison, Peter (2007): Objectivity. New York: Zone Books.

Doane, Mary Ann (2005): Zeitlichkeit, Speicherung, Lesbarkeit. Freud, Marey und der Film. In: Schmidgen, Henning (Hg.): Lebendige Zeit. Berlin: Kadmos, S. 280-313.
Drummond, Ann E. (2006): The Role of Steroids in Follicular Growth. In: Reproductive Biology and Endocrinology, vol.4, no. 16, pp. $1-11$

Ehrlich, Paul/Lazarus, Adolf (1900): Histology of the Blood: Normal and Pathological. In: Himmelweit, Fred/Marquardt, Martha/Dale, Henry (eds.): Collected Papers of Paul Ehrlich, vol. 1. New York: Pergamon Press 1956, pp. 181-268.

Fol, Hermann (1877): Sur le commencement de l'hénogénie chez divers animaux. In: Archives des sciences physiques et naturelles, no. 58 , pp. $439-472$.

Franklin, Sarah (2007): Dolly Mixtures: The Remaking of Genealogy. Durham, NC: Duke University Press.

Furnes, Bjarte/Schimenti, John (2007): Fast Forward to New Genes in Mammalian Reproduction. In: Journal of Physiology, vol. 578 , no. 1, pp. 25-32.

Ginzburg, Carlos (1980): Morelli, Freud and Sherlock Holmes: Clues and Scientific Method. In: History Workshop Journal, vol. 9, pp. $5-36$.

Gooday, Graeme (1991): >Nature in the Laboratory: Domestication and Discipline with the Microscope in Victorian Life Science. In: The British Journal for the History of Science, vol. 24, no. 3 , pp. 307-341.

Griesemer, James (2002): SPACE $\Leftrightarrow$ TIME. Temporality and Attention in Iconographies of the Living. In: Schmidgen, Henning (ed.): Experimental Arcades: The Materiality of Time Relations in Life Sciences, Art, and Technology (1830-1930). Preprint 226. Berlin: Max Planck Institute for the History of Science, pp. 45- 57

Griesemer, James/Yamashita, Grant (2005): Zeitmanagement bei Modellsystemen. Beispiele aus der Evolutionsbiologie. In: Schmidgen, Henning (Hg.): Lebendige Zeit. Berlin: Kadmos, S. $280-313$

Hacking, Ian (1985): Do We See Through a Microscope? In: Van Fraassen, Bas C./Churchland, Paul M./Hooker Clifford A (eds.): Images of Science. Chicago: Chicago University Press, pp. $132-152$.

Hennig, Jochen (2006): Die Versinnlichung des unzugänglichen - Oberflächendarstellungen in der zeitgenössischen Mikroskopie. In: Heßler, Martina (Hg.): Konstruierte Sichtbarkeiten. Wissenschafts- und Technikbilder seit der Frühen Neuzeit. München: Wilhelm Fink, S. 99-116. 
Hertwig, Oscar (1876): Beiträge zur Erkenntnis der Bildung, Befruchtung und Theilung des thierischen Eies. In: Morphologisches Jahrbuch, Nr. 1, H. 3, S. 347-432.

Heßler, Martina (2006): Von der doppelten Unsichtbarkeit digitaler Bilder. In: Zeitenblicke, Jg. 5, Nr. 3. Online unter: www.zei tenblicke.de/2006/3/Hessler (zuletzt aufgerufen: 11. März 2015)

Hine, Christine (2006): Databases as Scientific Instruments and Their Role in the Ordering of Scientific Work. In: Social Studies of Science, vol.36, no. 2, pp. $269-298$.

Hopwood, Nick/Schaffer, Simon/Secord, Jim (2010): Seriality and Scientific Objects in the Nineteenth Century: Introduction In: History of Science, vol.161, no.48, pp. 251-285.

Jelinski, Scott A. et al. (2007): The Rat Epididymal Transcriptome: Comparison of Segmental Gene Expression in the Rat and Mouse Epididymides. In: Biology of Reproduction, vol.75, no.4, pp. $561-570$.

Johannsen, Wilhelm (1911): The Genotype Conception of Heredity. In: The American Naturalist, vol.45, no. 531, pp. 129-159.

Kitano, Hiroaki (ed.) (2001): Foundations of Systems Biology Cambridge, MA: MIT Press.

Kittler, Friedrich (1986): Gramophone, Film, Typewriter. Berlin: Brinkmann und Bose.

Kogge, Werner (2012): Script, Code, Information: How to Differentiate Analogies in the »Prehistory « Molecular Biology In: History and Philosophy of the Life Sciences, vol.34, no.4, pp. $603-635$.

Kölliker, Albert von (1895): Die Bedeutung der Zellenkerne für die Vorgänge der Vererbung. In: Zeitschrift für wissenschaftliche Zoologie, Jg. 42, Nr. 1, S.3-50.

Landecker, Hannah (2002): New Times for Biology: Nerve Cultures and the Advent of Cellular Life in Vitro. In: Studies in History and Philosophy of Biological and Biomedical Sciences, vol. 33 , no. 4 , pp. $667-694$.

Landecker, Hannah (2005): Living Differently in Time: Plasticity, Temporality and Cellular Biotechnology. In: Culture Machine, vol.7. Online: http://www.culturemachine.net (last access: 25 September 2013).

Landecker, Hannah (2007): Culturing Life: How Cells Became Technologies. Cambridge, MA: Harvard University Press.
Latour, Bruno (1986): Visualization and Cognition: Thinking with Eyes and Hands. In: Knowledge and Society: Studies in the Sociology and Culture Past and Present, vol. 6, pp.1-40.

Lin, Ying-Hung et al. (2005): Isochromosome of Yp in a Man with Sertoly-cell-only Syndrome. In: Fertility and Sterility, vol. 83, no. 3, pp. $764-766$.

Liu, Zenshan et al. (2011): Comparative Analysis on MRNA Expression Level and Methylation Status of DAZL Gene between Cattle Yaks and Their Parents. In: Animal Reproductive Science, vol. 126 , no. $3-4$, pp. $258-264$.

Lynch, Michael (1985): Discipline and the Material Form of Images: an Analysis of Scientific Visibility. In: Social Studies of Science, no. 15 , pp. 37-66.

Merz, Martina (2006): Locating the Dry Lab on the Lab Map. In: Lenhard, Johannes/Küppers, Günter/Shinn, Terry (eds.): Simulation: Pragmatic Constructions of Reality. Dordrecht: Springer, pp. 155-172.

Morgan, Mary S. (2003): Experiments without Material Intervention: Model Experiments. In: Radder, Hans (ed.): The Philosophy of Scientific Experimentation. Pittsburgh, PA: University of Pittsburgh Press, pp. 216-235.

Morgan, Mary S. (2005): Experiments Versus Models: New Phenomena, Inference and Surprise. In: Journal of Economic Methodology, vol.12, no. 2, pp.317-329.

Nowotny, Helga/Testa, Giuseppe (2010): Naked Genes. Reinventing the Human in the Molecular Age. Cambridge, MA: MIT Press.

Rheinberger, Hans-Jörg (2003): Präparate ->Bilder`ihrer selbst. Eine bildtheoretische Glosse. In: Bredekamp, Horst/Bruhn, Matthias/Werner, Gabriele (Hg.): Bildwelten des Wissens. Kunsthistorisches Jahrbuch für Bildkritik, Bd. 1.2: Oberflächen der Theorie. Berlin: Akademie Verlag.

Rheinberger, Hans-Jörg/Müller-Wille, Staffan (2009): Vererbung: Geschichte und Kultur eines biologischen Konzepts. Frankfurt a. M.: Fischer Taschenbuch Verlag.

Schaffner, Kenneth (2007): Theories, Models, and Equations in Systems Biology. In: Boogerd, Fred C. et al. (eds.): Toward a Philosophy of Systems Biology. Amsterdam: Elsevier, pp. 145-162.

Schickore, Jutta (2007): The Microscope and the Eye: A History of Reflections, 1740-1870. Chicago: University of Chicago Press.

Schleiden, Matthias Jacob (1842/43): Grundzüge einer wissenschaftlichen Botanik. Leipzig: Engelmann. 
Schmidgen, Henning (2004): Pictures, Preparations, and Living Processes: The Production of Immediate Visual Perception (Anschauung) in Late-19th-century Physiology. In: Journal of the History of Biology, vol. 37, no.3, pp.477-513.

Schmidgen, Henning (2007): Zukunftsmaschinen. In: Rechtsgeschichte - Legal History. Zeitschrift des Max-Planck-Instituts für Europäische Rechtsgeschichte, Nr. 10, S. 51-62.

Schröter, Jens (2004): Das Ende der Welt. Analoge vs. digitale Bilder - mehr und weniger »Realität «? In: ders./Alexander Böhnke (Hg.): Analog/Digital - Opposition oder Kontinuum? Zur Theorie und Geschichte einer Unterscheidung (Medienumbrüche, 2). Bielefeld: transcript, S.7-30.

Sermon, Karen (2011): Interview. Tonaufnahme, 26. Mai.

Shima James E. et al. (2004): The Murine Testicular Transcriptome: Characterising Gene Expression in the Testis during the Progression of Spermatogenesis. In: Biology of Reproduction, no. 71, pp. 319-330.

Stevens, Nettie Maria (1905): Studies in Spermatogenesis with Especial Reference to the »Accessory Chromosome «. Washington D.C.: Carnegie Institution of Washington. Online: http://www. archive.org/details/studiesinspermato1stevrich (last access: 15 May 2015).

Strelchenko, Nick et al. (2006): Reprogramming of Human Somatic Cells by Embryonic Stem Cell Cytoplast. In: Reproductive BioMedicine Online, vol. 12, no. 1, pp. 107-111. Online: www.rbonline.com/Article/2017 (last access: 1 August 2015).
Wahrig-Schmidt, Bettina (1994): Das >geistige Auge<des Beobachters und die Bewegungen der vorherrschenden Gedankendinge. In: Hagner, Michael/Rheinberger, Hans-Jörg/Wahrig-Schmidt, Bettina (Hg.): Objekte, Differenzen und Konjunkturen. Berlin: Akademie Verlag, S. 23-47.

Waldeyer, Wilhelm (1888): Über Karyokinese und ihre Beziehun gen zu den Befruchtungsvorgängen. In: Archiv für mikroskopische Anatomie und Entwicklungsmechanik, Jg. 32, Nr. 1, S. 1-122.

Wang, Ning et al. (2011): Altered Expression of Armet and Mrlp51 in the Oocyte, Preimplantation Embryo, and Brain of Mice Following Oocyte in Vitro Maturation but Postnatal Brain Development and Cognitive Function Are Normal. In: Reproduction, vol. 142 , no. 3 , pp. $401-408$.

Wellman, Janina (2011): Science and Cinema. In: Science in Context, vol. 24 , no. 3 , pp. $311-328$.

Wilkins-Haug, Louise (2009): Epigenetics and Assisted Reproduction. In: Current Opinion in Obstetrics and Gynecology, vol. 21, no.3, pp. 201-206.

Wilson, Edmund B. (1895): An Atlas of the Fertilisation and Karyokinesis of the Ovum. New York: Columbia University Press.

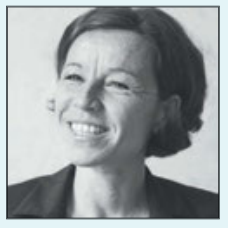

bettina.bock.v.wuelfingen@hu-berlin.de

Wissenschaftliche Mitarbeiterin

Basisprojekt: Gender \& Gestaltung

Disziplin: Biologie, Kulturwissenschaft

Bettina Bock von Wülfingen ist Kulturwissenschaftlerin mit einem Hintergrund in Biologie und Gesundheitswissenschaft und hat am Institut für Kulturwissenschaft der Humboldt-Universität zu Berlin 2012 mit der Schrift Economies and the Cell - Heredity and Conception Around 1900 and 2000 habilitiert. Ihre derzeitige Forschung am Exzellenzcluster Bild Wissen Gestaltung gilt der bildlichen Modellierung von Beziehungen unterhalb und oberhalb der zellulären Ebene und dem Übergang von Reproduktion und Produktion zu (Re-)Generation in Biowissenschaften und Medizin. 


\section{Wolfgang Schäffner}

\section{Interdisziplinäre Gestaltung}

\section{Einladung in das neue Feld einer Geistes- und Materialwissenschaft}

\section{Carbon Flatland}

Vor genau 10 Jahren hat ein Goldrausch in ein ebenso neues wie altes Reich des Zweidimensionalen begonnen. Nach Jahrtausenden des Trainings, die räumliche Welt durch symbolische Operationen von Bild, Schrift und Zahl auf 2D-Oberflächen zu bringen, geschah 2004 etwas, das bis dahin für unmöglich gehalten worden war: Die bloße Fläche tauchte endlich selbst im Reich der Dinge auf, als eine materiale zweidimensionale Ebene mit der minimalen Dicke einer Carbonatomschicht - ein geradezu euklidischer Traum, ein hauchdünnes, durchsichtiges und flexibles Gitter aus Hexagonen und dennoch hundertmal fester als Stahl. Materiale Geometrie und verkörperte Quantendynamik: Graphen ist ein Ereignis, das in der Physik eine Revolution auslöste, als Blatt schlechthin, in dem Physik und Kultur in eigentümlicher Weise zusammenfallen (Abb. 1). Denn, so schreibt einer seiner beiden Entdecker Andre Geim, »what is probably more unexpected is the news that every time someone scribes a line with a pencil, the resulting mark includes bits of the hottest new material in physics and nanotechnology: graphene «. 'Es ist die dünne »[m]other of all graphitic forms «, da mit nur wenigen Faltungen daraus so irrwitzige Dinge wie nanotubes und buckyballs entstehen (Abb.2). All dies resultiert also aus einem einfachen Verfahren, »nothing more sophisticated than drawing by a piece of

1 Geim/Kim 2008, 90. 


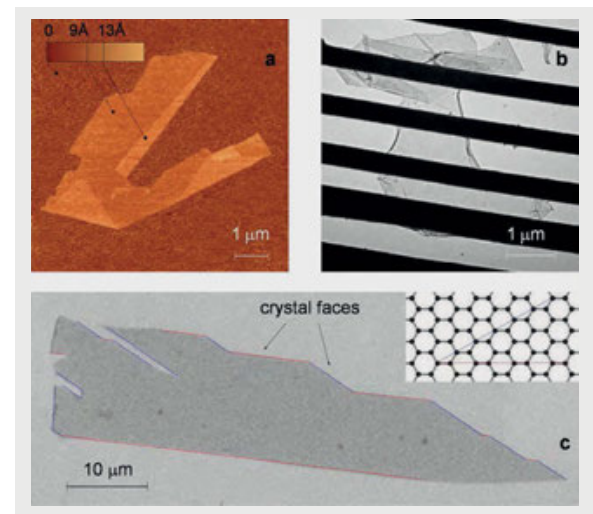

Abb. 1: »One-atom-thick single crystals: the thinnest material you will ever see.«

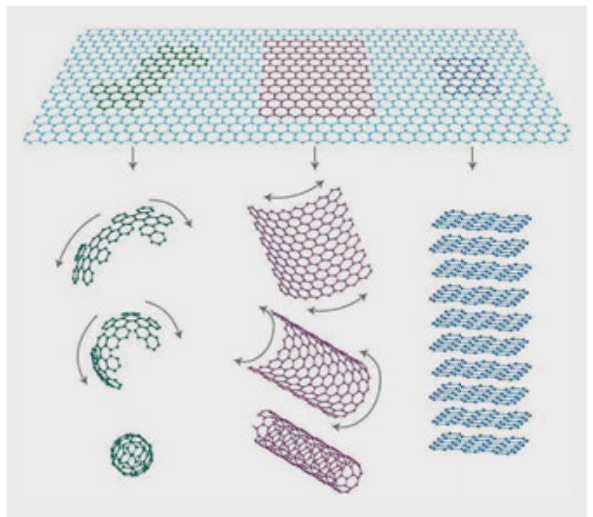

Abb. 2: » Mother of all graphitic forms. Graphene is a $2 \mathrm{D}$ building material for carbon materials of all other dimensionalities. It can be wrapped up into oD buckyballs, rolled into $1 \mathrm{D}$ nanotubes or stacked into 3D graphite.«

graphite or its repeated peeling with adhesive tape until the thinnest flakes are found «. ${ }^{2}$ Bei dieser überraschend simplen Technik jedoch lag eine besondere Schwierigkeit darin, die produzierte minimale Schicht tatsächlich sichtbar zu machen und aufzufinden wie eine Nadel in einem Heuhaufen von Tausenden dickeren Grafitflocken: Denn weder in Rastersonden- noch in Rasterelektronenmikroskopen ist sie zu sehen, nur in einem optischen Mikroskop, wenn man sie auf einen Siliziumwafer als Grundplatte legt, oder neuerdings auch über die Raman-Spektroskopie. Verborgen in der Bleistiftschrift also ruhte diese Revolution, eine Art Physik des Schreibens, die damit auch ein Ereignis im Feld der Geisteswissenschaften und des Grafikdesigns darstellt. Grafit, das man bei seiner Entdeckung im 16. Jahrhundert in England noch für das Bleimineral Plumbago hielt, wurde zur Grundlage des modernen Bleistifts (Abb.3). Erst gegen Ende des 18. Jahrhunderts wies man nach, dass es sich dabei um reinen Kohlenstoff handelt, weshalb der Mineraloge Abraham Gottlob Werner das Material >Graphit< nannte, abgeleitet vom griechischen graphein, schreiben. ${ }^{3}$ Wenn der Naturwissenschaftler und Experimentator der Royal Society of London Robert Hooke solche Punkte und Linien bereits in seiner Micrographia von 1665 untersucht (Abb.4), beginnt die Geschichte der Schrift unter dem Mikroskop beim Punkt, der als »great splash of London dirt« ${ }^{4}$ erscheint, und sie ist nun beim Graphen angekommen. Dieser Zoom in andere Dimensionen des Schreibens war für den Physiker Richard Feynman 1959 der Grund, im Rahmen des Vortrags There is Plenty of Room at the Bottom ${ }^{5}$ am California Institute of Technology in ein neues Feld der Physik einzuladen. Die Herausforderung, die er

2 Geim/Novoselev 2007, 3.

3 Siehe Petroski 2003.

4 Hooke $1665,3$.

5 Feynman 1960. 


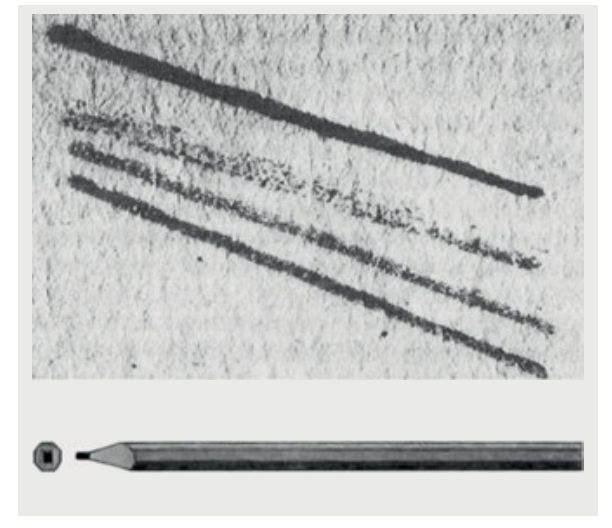

Abb. 3: Ein früher Bleistift und die Striche, die er auf dem Papier hinterlässt.

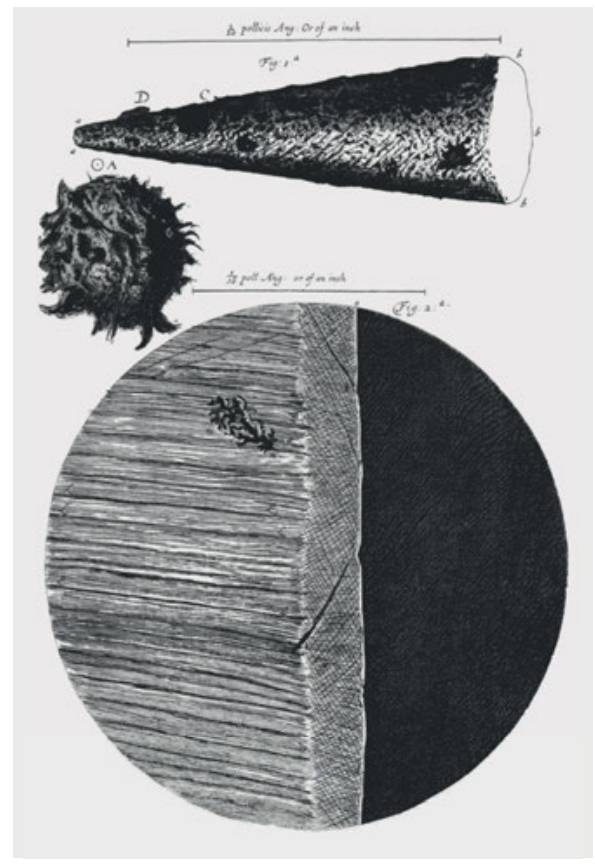

Abb. 4: Abbildung von Nadelspitze, Punkt und Papier aus Robert Hookes Micrographia.

damals sah, lag ebenfalls in einer eigentümlichen Verschmelzung von Kulturtechniken und Festkörperphysik. Denn einführend stellte er die schlichte Frage: »Why cannot we write the entire 24 volumes of the Encyclopedia Brittanica on the head of a pin? « ${ }^{6}$ Sein Blick in den Zoom einer materialen Miniaturisierung lotete die Grenzen der Verkleinerung des Schreibens und Zeichnens von elektronischen digitalen Schaltungen aus. Zugleich wurde Physik zu Hardware-Design, indem Atome als Bausteine von Symboloperationen fungierten. Dies brachte Geisteswissenschaftler wie Friedrich Kittler dazu, die Makroebene des Schreibens zu verlassen und statt Literaturwissenschaft eine Geschichte und Theorie der Soft- und Hardware zu betreiben - mit dem Ziel, dem Schreiben vom Typewriter des 19. Jahrhunderts in die Regionen zu folgen, wo es seine radikalsten Innovationen bewirken sollte. ${ }^{7}$

Die neue Hardware-Physik führte Richard Feynman aber auch unmittelbar in die Biologie, die damals in den Bannkreis der Informationstheorie geriet: ${ }^{8}$ Denn die Molekularbiologie zeigte Feynman, dass es hierbei nicht nur um Schreiben, Verarbeiten und Speichern von Informationen geht; vielmehr tun biologische Moleküle etwas damit. Sie agieren; sie repräsentieren einen Code nicht nur, sondern führen ihre Codierungen als kleine Maschinen auch aus, und dies nicht

6 Ebd., 22.

7 Siehe Kittler 1986 sowie ders. 1993.

8 Vgl. dazu Yockey/Platzman/Quastler 1958. 
mehr virtuell, sondern als materiale analoge Codes. Entscheidend für Feynman war nun, dass technische Elemente, biologische Moleküle und physikalische Materie im Nanobereich nach denselben Prinzipien funktionieren, die für die Gestaltung bereitstehen, und dafür gibt es eben, wie er sagt, » plenty of room at the bottom «. ${ }^{9}$

In den Labors ist diese Science Fiction mittlerweile Wirklichkeit der Forschung geworden: Biologische Materialien werde als Hardware analysiert; materiale Strukturanalysen und Gestaltungen ebnen die Grenzen von Physik, Chemie, Mechanik und Biologie ein; es findet ein regelrechter Design-Turn ${ }^{10}$ in diesen Wissenschaften statt. Artefakte, Maschinen und natürliche Objekte werden ununterscheidbar, und nicht zuletzt unternehmen die Geisteswissenschaften neue Akteur-, Struktur- und Bildforschungen. So kann man das Graphen neben den Maschinen, die in biologischen Materialien gesehen werden und die Materialforschung prägen, als weiteres Indiz dafür nehmen, dass ein völlig neuer Blick auf die materielle Welt entsteht: Was vormals als passive, opake Materialität galt, wird nun zum Motor, Sensor und Code einer dynamischen Strukturenwelt. Es ist, als seien die digitalen Algorithmisierungen im Bereich der Materie selbst angekommen. Nun geht es darum, das Analoge unserer materialen Welt als operatives System neu zu entdecken.

Diese Situation in ihrer ganzen Radikalität und Komplexität zu erkennen und daraus Konsequenzen zu ziehen, ist für uns die konkrete gegenwärtige Herausforderung. Und darauf zielt die Einladung zu einer Forschung, die wir uns an diesem Cluster als interdisziplinäre Gestaltung vornehmen: eine grundlegende Kooperation von Wissenschaft und Gestaltung, die man als Geistes- und Materialwissenschaft bezeichnen kann. Nach einem Blick auf die institutionellen Bedingungen öffentlicher Forschungsstrukturen für dieses neue Gebiet werde ich im Folgenden ein konkretes Arbeitsfeld des Clusters vorstellen, das die großen Anforderungen an eine integrative interdisziplinäre Arbeit erkennen lässt.

\section{Wissenschaft und Gestaltung}

Unser universitäres System ist für das neue Feld einer Geistes- und Materialwissenschaft nicht unbedingt vorbereitet: Die Ausdifferenzierung von Technischen Universitäten, Kunsthochschulen und Gesamtuniversitäten, in denen die Geistes- von den Naturwissenschaften getrennt sind, hat eine neuere Geschichte. Sie beginnt um 1800 mit der Gründung der École Polytechnique in Paris: Die Géométrie descriptive (1798) von Gaspard Monge bildete die operative Gestaltungstechnik für alle Disziplinen, die an der École unterrichtet wurden." Parallel zu diesem Modell der Ingenieurausbildung erfolgte die humboldtsche Reorganisation der Universität - man könnte sogar sagen: als Reaktion gegen diese neue Macht technischen Wissens. Statt darstellender Geometrie bildete hier die Philosophie die Basis, an die Stelle eines Gaspar Monge trat daher

9 Feynman 1959.

10 Siehe Schäffner 2010.

11 Siehe Monge 1798. 
Johann Gottlieb Fichte mit seiner Wissenschaftslehre, die alle Wissenschaften aus der Selbsttätigkeit des Subjekts ableitet. ${ }^{12}$ Alexander von Humboldt ging es aber auch darum, die sich neu formierenden Naturwissenschaften wie Physik oder Chemie in den Raum der Universität einzubinden. Deshalb wurden in der Folge gerade solche Forscher an die Berliner Universität berufen, die ihre eigenen Labore mitbrachten.

Vor diesem Hintergrund zeichnet sich die Herausbildung von vier sehr verschiedenen Wissenskulturen ab: die beiden Felder der Geistes- und Naturwissenschaften, die Ingenieurwissenschaften und die Gestaltungsdisziplinen an den Kunsthochschulen. Dieses Modell bestimmt noch immer unsere Gegenwart und erschwert ein integratives Verhältnis von Wissenschaft und Gestaltung.

Unser Interdisziplinäres Labor Bild Wissen Gestaltung knüpft deshalb nicht zufällig an die Arbeiten von Hermann von Helmholtz an, der gerade in entscheidenden Phasen der Trennung von Geistes- und Naturwissenschaften deren engen Zusammenhang hervorhob. So 1877 in seiner Antrittsrede als Präsident der Berliner Universität:
»In der That, so sehr auch die Objecte, die Methoden, die nächsten Ziele naturwis- senschaftlicher Untersuchungen von denen der Geisteswissenschaften äusserlich unterschieden sein mögen, und so fremdartig ihre Ergebnisse, so fernliegend das Interesse daran oft denjenigen Männern erscheinen mag, die gewöhnt sind, sich nur mit den unmittelbaren Aeusserungen und Erzeugnissen des Geisteslebens zu be- schäftigen: so besteht doch [...] in Wahrheit die engste Verwandtschaft im innersten Wesen der wissenschaftlichen Methode, wie in den letzten Zielen beider Klassen von Wissenschaften. $\ll^{13}$

Vor genau 50 Jahren schrieben Tomás Maldonado und Gui Bonsiepe mit Wissenschaft und Gestaltung an der Ulmer Hochschule für Gestaltung ein ebenso historisches wie uneingeholtes Programm für die Einbeziehung der wichtigsten neuen Wissenschaften: Topologie, Strukturforschung (sie nennen die Ingenieure und Architekten Richard Buckminster Fuller, Pier Luigi Nervi, Eduardo Torroja, Félix Candela und Konrad Wachsmann), Informationstheorie, Kybernetik und Ergonometrie sind die Bereiche, die Maldonado und Bonsiepe für eine wissenschaftliche, technische und methodologische Erneuerung der Entwurfspraxis heranziehen. ${ }^{14}$ Zwar hatten die Lehrmodelle und resultierenden Entwürfe - etwa für Braun und Lufthansa, aber auch Otl Aichers Gestaltung der Olympischen Spiele in München 1972 - weltweit großen Erfolg; doch im Rahmen heftiger politischer Diskussionen erfuhr die konkrete Integration von Wissenschaft

\footnotetext{
12 Siehe Fichte $1994 / 95$.

13 Helmholtz 1877, 5 f. Das Hermann von Helmholtz-Zentrum für Kulturtechnik der Humboldt-Universität, das als Zentralinstitut den Charakter einer neuartigen integrativen Fakultät hat, schließt an diese Berliner Geschichte an und überführt sie in eine neue Phase, in der nun aber natürlich auch Frauen eine wichtige Rolle spielen.

14 Siehe Maldonado/Bonsiepe 1964.
} 
und Gestaltung mit der Schließung der Ulmer Hochschule durch die Landesregierung 1968 ein abruptes Ende. ${ }^{15}$

Obwohl die skizzierten Programme von Feynman (1959) sowie Maldonado und Bonsiepe (1964) in keinem direkten Zusammenhang stehen, bilden sie Bestandteile einer transversalen Untersuchung von Strukturen, die Festkörperphysik, Hardware-Design, Biologie, Architektur und Produktgestaltung ebenso vereint wie die Felder des klassischen Strukturalismus. Doch eine derartig naheliegende transversale Strukturforschung betrieb man in den 1960er-Jahren selbst in Paris, dem damaligen Zentrum des Strukturalismus, nicht. Dies geschah tatsächlich eher in Ulm oder am Massachusetts Institute of Technology (MIT) durch den Gestalter György Kepes, der 1965 den Band Structures in Art and in Science herausgab, in dem sich neben Fuller und Nervi auch Metallurgen wie Cyril Stanley Smith und Physiker wie Lancelot White finden. ${ }^{16}$ An solche Konstellationen schließt die interdisziplinäre Struktur unseres Clusters an.

\section{Unfoldome}

Vor diesem Hintergrund wird deutlich, in welch neuer Situation wir uns 50 Jahre später befinden und inwiefern die Physik des Graphens neben der Biomaterialforschung einem New Materialism in der Philosophie ${ }^{17}$ oder einer Geschichte des analogen Codes $^{18}$ ein Feld konvergenter Fragestellungen eröffnet: In diesem Sinne diente das am Cluster durch den Physiker Jürgen Rabe untersuchte Graphen als Einstieg in ein neues Feld der Forschung, in dem Natur- und Geisteswissenschaften ebenso wie Gestaltungsdisziplinen in einer integrierten Forschungsform vereint sind. Es handelt sich hierbei um ein Forschungsgebiet, bei dem es um die Idee einer aktiven Materie geht, die zugleich Codecharakter hat. Diese dynamischen materialen Strukturen dienen uns dazu, die Geschichte der Digitalisierung und der Genese von Algorithmen und Datenstrukturen auf die Materie selbst zu übertragen, die nun nicht mehr als passiver, opaker Träger erscheint, sondern selbst zum Akteur, zu einer neuen Art Maschine werden kann: ${ }^{19}$ Dabei stehen Prozesse der Faltung, der 1D-, 2D- und 3D-Codierung von räumlichen Strukturen im Zentrum, die in unterschiedlichen Maßstäben untersucht werden. ${ }^{20}$ Während beim Graphen die Physik in Regionen des Grafikdesigns eindringt, stoßen umgekehrt die symbolischen Operationen des Druckens in Dimensionen der Physik vor und werden nach Ludger Hovestadt

$15 \mathrm{Vgl}$. dazu allgemein Rinker 2003.

16 Siehe Kepes 1965.

17 Siehe De Landa 1997.

18 Siehe Schäffner 2016.

19 Für diese Übertragung der digitalen Algorithmisierung in die Agentivierung von Materialien selbst war die Mitarbeit des Informatikers und Mathematikers Bernd Mahr ein Glücksfall. Kaum einer kannte so wie er die Genese der digitalen Algorithmen, die nun anstehen, in ganz neuer Weise im Material selbst nochmals erfunden zu werden. Nach seinem Tod bleiben uns seine unersetzlichen Anregungen und seine Schriften. Siehe etwa Mahr u. a. 2001.

20 Vgl. Demaine/O'Rourke 2007. 
zu »printed physics «:21 Der 3D-Druck, der sich aus der flatness des Papiers erhebt, überkreuzt sich damit eigentümlich mit dem

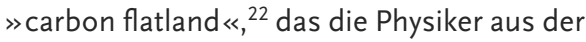
3D-Welt isolieren.

Was sich einerseits in dem extrem leicht faltbaren Graphen als dynamische Mechanik abspielt, kann auch auf Makroebene an organischen Blattstrukturen untersucht werden. Diese Biomaterialforschung - am Cluster betrieben durch Peter Fratzl, John Dunlop, Richard Weinkamer ${ }^{23}$ und Lorenzo Guiducci - versteht dabei Pflanzenblätter als Hardware, die in 2D-patterns die 3D-Entfaltung oder -Auffaltung ihrer Blattfläche codiert (Abb.5). Modellierungen dieser Prozesse führen einerseits in die Morphologie unserer Biologen Gerhard Scholtz und Thomas Stach, deren Wissen über die Strukturen von Pflanzen und Lebewesen dabei in Fragestellungen der Mechanik und analogen Codierung übertragen wird. Denn der Blick dieser Materialingenieure erkennt in den Strukturen der organischen Materialien aktive Mechanismen, die als technische Lösungen adaptiv, energieeffizient und überaus flexibel sind.
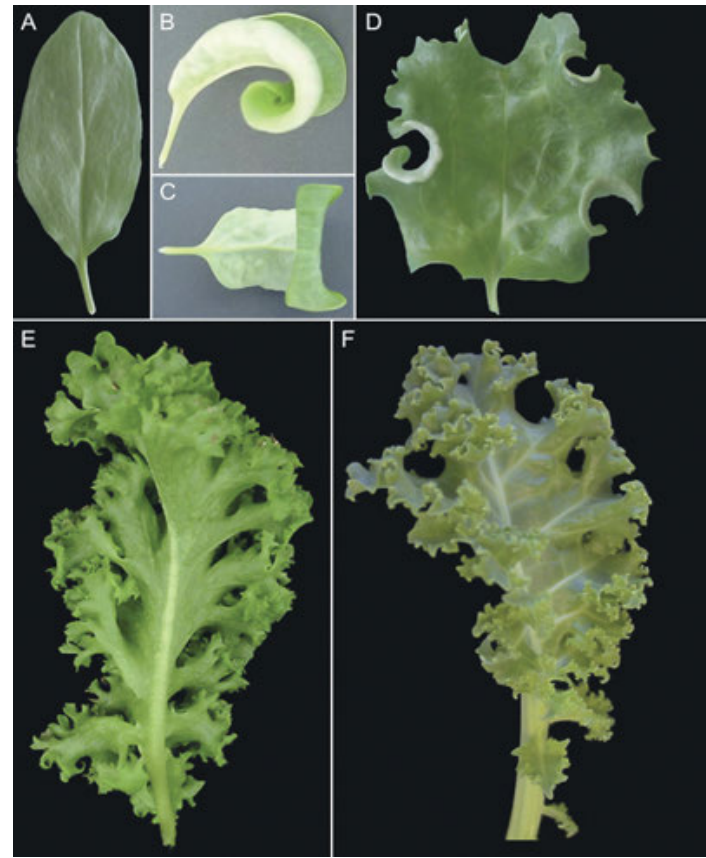

Abb. 5: »Genetic basis of the emergence of negative curvature in leaves «.

Diese Kehrtwende von einer Implementierung technischer Intelligenz als dem klassischen Paradigma des Ingenieurwesens zum Aufsuchen technischer Lösungen in der Natur - als neuem Soft Engineering - stellt andererseits eine radikale Herausforderung dar. Vergleicht man sie mit dem Entwerfen im Produktdesign oder in der Architektur, dann wird deutlich, wie sehr sich das Vorgehen von Gestaltenden verändert, wenn Strukturen nicht mehr durch Entwurfsstrategien im Material realisiert werden, sondern diese umgekehrt aus diesem selbst, aus einem »architectured material $\ll,{ }^{24}$ entwickelt werden: Elemente der Architektur sind nun aktive Strukturen des Materials selbst, dessen patterns neue Formen des Bauens bedingen. 
Ein Blick auf den Prozess des Faltens kann das verdeutlichen: Faltungen sind 1D- oder 2D-Operationen, die 3D-Strukturen hervorbringen. Seit den Arbeiten des Bauhaus-Professors Josef Albers oder Richard Buckminster Fullers ${ }^{25}$ sind diese Faltungen als elementare Gestaltungsprozesse von Produktdesign und Architektur untersucht worden. Insbesondere Fuller leitet aus seiner synergetischen Geometrie der Faltung innovative dynamische Strukturen ab. Man könnte dieses gesamte Feld mit dem Biochemiker Vladimir Uversky als unfoldome ${ }^{26}$ als System von Faltungen bezeichnen. Die neuere Proteinforschung zeigt, dass entgegen früherer Auffassungen sehr viele Proteine keine stabile Struktur besitzen: »Structural flexibility and plasticity from the lack of a definite-ordered $3 D$ structure are believed to represent a major functional advantage for these proteins. ${ }^{27}$ Faltung bildet den operativen Kern von Strukturen. Proteinfaltung, ${ }^{28}$ das faltbare Gewebe des Graphens, die dynamischen Strukturen von organischen Materialien - all diese Elemente in nano-, meso- und makroskalischen Bereichen werden gegenwärtig nicht zufällig mit Origami-Geometrie betrieben: In diesem sehr jungen Bereich der Mathematik geht es um Konstruktionen, die nicht mit Lineal und Zirkel, sondern durch das Falten von Papier erzeugt werden. Dieses Verfahren stammt aus der alten Tradition des Papierfaltens in Japan und Ägypten und erlebt unter dem Namen Origami vor allem seit der Mitte des 20. Jahrhunderts eine regelrechte Revolution, wobei die Verfahrensschritte praktisch formalisiert und auch mathematisiert werden. Bei der aktuellen Axiomatisierung wird deutlich, dass es sich dabei um eine sehr innovative Geometrie handelt, in der etwa die klassischen griechischen sogenannten Delischen Probleme der Würfelverdoppelung und der Dreiteilung des Winkels leicht zu lösen sind. Diese neue Origami-Mathematik knüpft dabei an Mathematisierungen des Papierfaltens an, die im späten 19. Jahrhundert von dem Deutschen Hermann Wiener als Theorie der Umwendungen (1890) und dem Inder Sundara Row als Geometric Exercises in Paper Folding (1893) entwickelt wurden (Abb. 6). ${ }^{29}$

Gegenwärtig gibt es DNA-Origami (Abb. 7), ${ }^{30}$ Pflanzen-Origami, das nicht mehr gefaltet werden muss, weil seine Blätter oder Samenkapseln sich selbst falten, ${ }^{31}$ Elastomer-Origami im Bereich der Soft Robotics, ${ }^{32}$ 3D-gedruckte Origami-Strukturen ${ }^{33}$ und nicht zuletzt Graphen-Origami, um nur einige Formen zu nennen.

25 Siehe Fuller 1975/79.

26 Uversky 2010.

27 Ebd.

28 Siehe Dill et al. 2008.

29 Wiener 1890; Row 1893. Diese operative Geometrie um 1900 untersuche ich am Cluster zusammen mit dem Mathematiker Michael Friedman. Vgl. dazu Friedman 2016. Karin Krauthausen und Joseph Vogl bearbeiten philosophische Perspektiven der Strukturen- und Faltungsgeschichte. Diese Überlegungen versuchen wir mit aktuellen Fragen der Mechanik von Faltungen in Blattstrukturen und Graphenoxidschichten zu verbinden, an denen Lorenzo Guiducci mit Peter Fratzl und John Dunlop sowie Mohammad Fardin Cholami mit Jürgen Rabe arbeiten.

30 Siehe Smith 2006: DNA-Origami als Coverstory der Nature.

31 Siehe Fratzl et al. 2013.

32 Siehe Martinez et al. 2012.

33 Siehe Ahn et al. 2010. 
เo

GEONETRIC EXERCISA

are on each side of it. We thus get isosceles triangles standing on a side of the square.

23. The middle line divides the isosceles triangle into two congruent right-angled triangles.

24. The vertical angle is bisected.

25. If we so take the point on the middle line, that

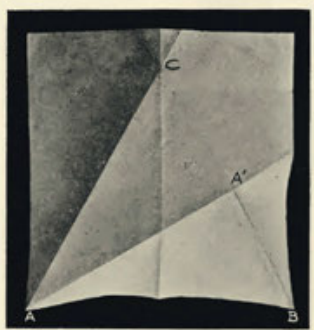

Fie na

its distances from two corners of the square are equal to a side of it, we shall obtain an equilateral triangle (Fig. 10). This point is easily determined by turning the base $A B$ through one end of it, over $A A^{\prime}$, until the other end, $B$, rests upon the middle line, as at $C$. 26. Fold the equilateral triangle by laying each
IN PAPES FOLOING

39. $A C^{\prime} A^{\prime} B$ is a rhombus. So are $C B A^{\prime} B^{\prime}$ and $C{ }^{\circ} C^{\prime} A$

40. $A^{\prime} B^{\prime}, B^{\prime} C^{\prime}, C^{\prime} A^{\prime}$ bisect the corresponding altitudes.

41. $C C^{3}+A C^{4}=C C^{3}+1 A C^{2}=A C^{3}$

$C C^{\prime 2}=1 A C^{2}$

$C C^{\prime}=1 \sqrt{3} \cdot A C=\frac{1}{3} \sqrt{3} \cdot A B$ $=0,806 \ldots \times A$

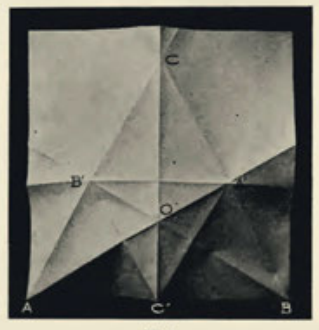

42. The $\triangle A B C=$ rectangle of $A C^{\prime}$ and $C C^{\prime \prime}$, i, e. $\downarrow A B \times\} \sqrt{3} \cdot A B=\frac{1}{2} \sqrt{3} \cdot A B^{n}=0.433 \ldots \times A B^{2}$. 43. The angles of the triangle $A C^{\circ} C$ are in the ratio of $1: 2: 3$, and its sides are in the ratio of $\sqrt{1}$ $\pm \sqrt{3}: \sqrt{4}$.

Abb. 6: Seiten aus Sundara Rows Geometric Exercises in Paper Folding.

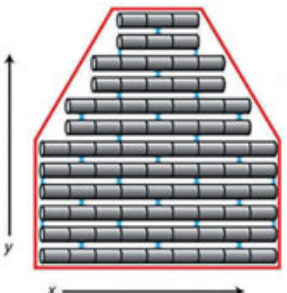

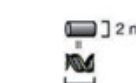

10.67 bases $~ 1$ turn $3.6 \mathrm{~nm}$ in length

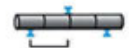

6 bases $=1.5$ turn between crossovers along a helix

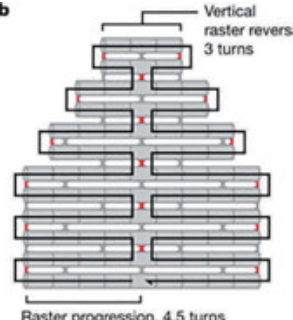

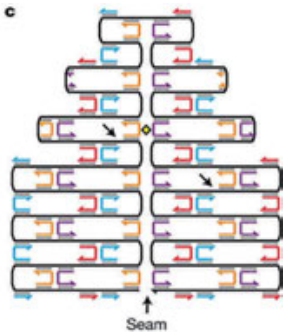

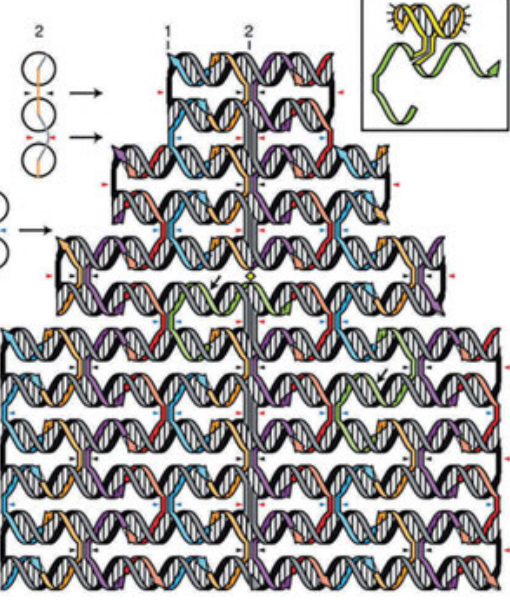

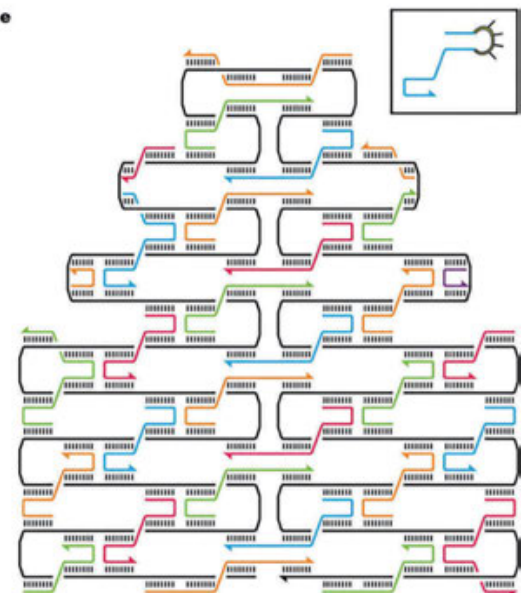

Abb. 7: »Design of DNA origami «. 

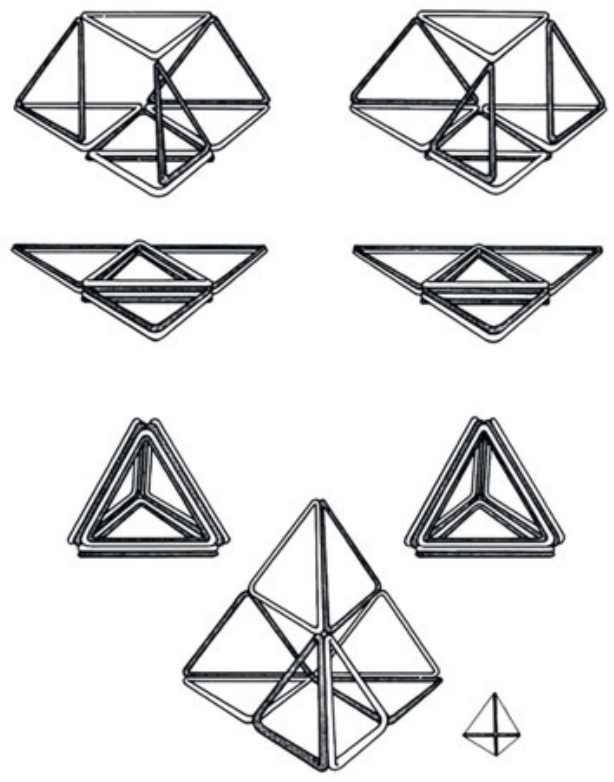

Abb. 8: Richard Buckminster Fuller, Jitterbug-Transformation.

Selbst Buckminster Fullers gefaltete Geometrie, ${ }^{34}$ die er mit so einfachen Elementen wie festen Stäben und Gummiverbindungen entwickelte und damit die Jitterbug-Transformation möglich machte, bei der die idealen platonischen Körper als Stadien eines Faltungsprozesses erscheinen, kann man in dieser neuen Weise analysieren (Abb. 8): Ausgehend von Friedrich Fröbels elementaren Faltaufgaben und der Faltgeometrie um 1900 über Fuller bis hin zur aktuellen Origami-Geometrie versuchen wir derzeit, eine Mathematik und Mechanik der Faltungen zu beschreiben, die als grundlegender analoger Code der aktiven Materie verstanden werden kann und als neuer Gestaltungsschauplatz des unfoldome dienen soll. Damit bewegen wir uns natürlich zugleich unmittelbar auf das Feld der Philosophie der Falte zu, die von Gottfried Wilhelm Leibniz über Michel Serres ${ }^{35}$ bis hin zu Gilles Deleuze ${ }^{36}$ führt. Die neuen self-folding Origami-Strukturen, die sich spontan durch Oberflächenspannungseffekte falten, sind kleine Maschinen, die sich als 2D-Blätter ausgedruckt auffalten und dann loslaufen: ${ }^{37}$ dies als vielleicht jüngstes Kapitel im unfoldome von active matter, philosophy of embodiment oder Horst Bredekamps Beispielen des Bildakts. 


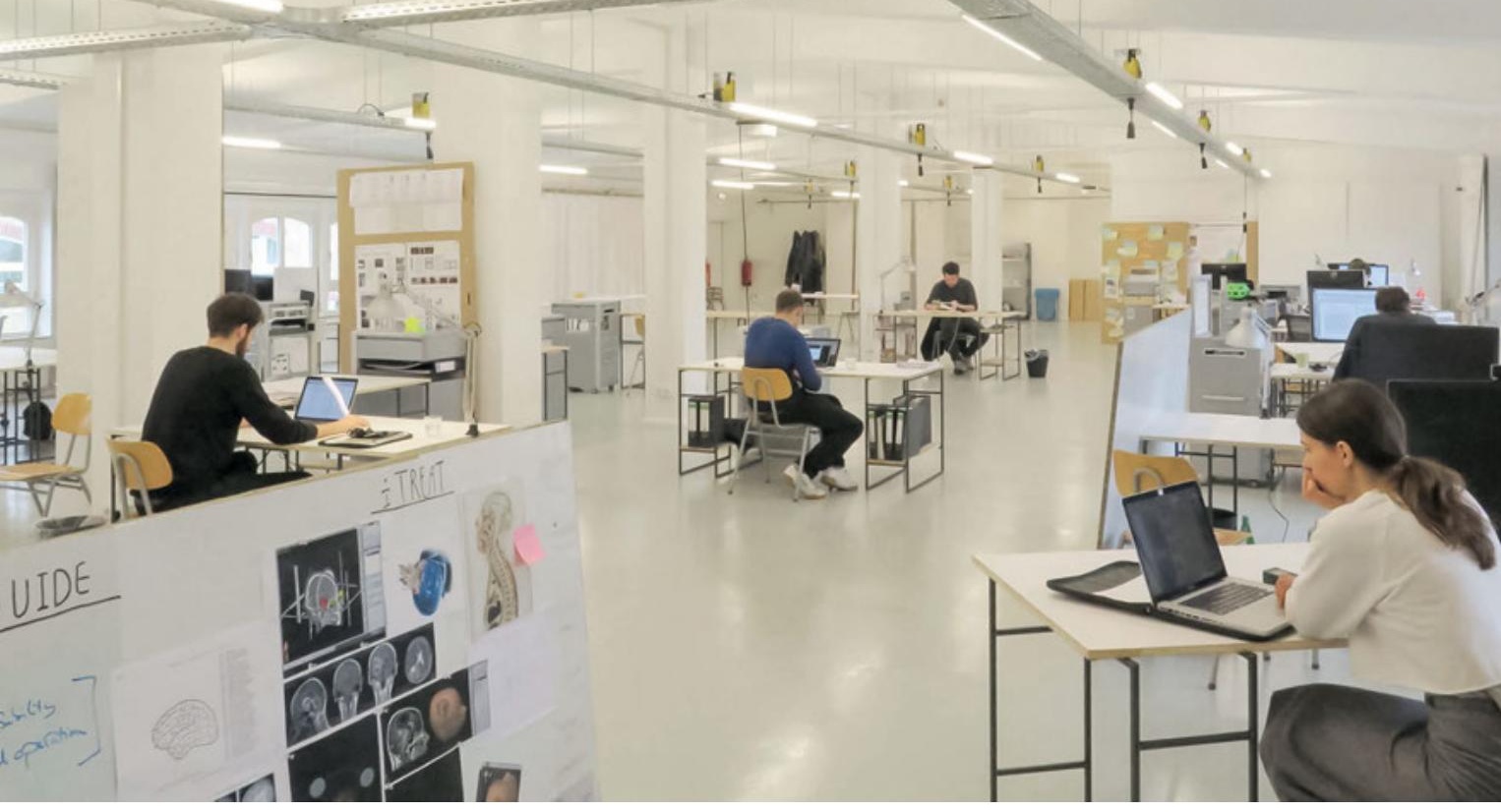

Abb. 9: Experimentalzone des Exzellenzclusters im vierten Stock der Sophienstraße.

Mein Gang durch die Faltungen des unfoldome versammelt somit eine lange Reihe von Disziplinen, die ein enges Gefüge bilden und im Exzellenzcluster Bild Wissen Gestaltung das gemeinsame Feld einer Geistes- und Materialwissenschaft ergeben. Hier ist meine Arbeit als Kultur- und Medienwissenschaftler an einer Geschichte und Theorie von Strukturen und analogen Codes mit den Labors der Materialforschung und der Gestaltung kurzgeschlossen sowie mit der Informatik und der Mathematik verbunden. Dazu braucht es aber auch ein interdisziplinäres Experimentallabor, das die Zusammen- und Auffaltung der unterschiedlichen Wissenskulturen als Gestaltungsprozess ermöglicht, als physischer Laborraum, der flexibel und intelligent reagieren kann, als Kommunikations- und Interaktionsraum, der eine integrative Funktion erhält (Abb. 9). Als historisch analysierender Geisteswissenschaftler geht es mir nicht zuletzt darum, die lange Reihe der Akteure von Euklid über Leibniz, Monge bis Fuller, Maldonado und Feynman gleichsam als Zeitgenossen in unsere Gegenwart zu falten - sie zusammenzubringen mit dem Biomaterialwissenschaftler Peter Fratzl, dem Physiker Jürgen Rabe, dem Mathematiker Jochen Brüning, dem Fuller-Experten Joachim Krausse, der Designerin Carola Zwick, ${ }^{38}$ dem Architekten Finn Geipel oder dem Literaturwissenschaftler Joseph Vogl, mit denen ich die Faltungen als neuen Code der Materie untersuche. Mit solchen topologischen Faltungen einer klassischen Zeitachse 
kann und muss man die längst überkommene Linearität der Geschichte durchbrechen. ${ }^{39}$ Dazu genügt es, die Dinge nebeneinanderzulegen und Konsequenzen daraus zu ziehen: Dies sehe ich als meine neue Aufgabe in der Geistes- und Materialwissenschaft, die wir aufbauen. Man kann es mit dem argentinischen Schriftsteller Jorge Luis Borges einen »jardin de los senderos que se bifurcan « ${ }^{40}$ nennen, einen Garten, dessen Pfade sich verzweigen. Denn Tomás Maldonado grüßt uns heute aus Buenos Aires, wo er mit uns ein gemeinsames interdisziplinäres Labor, das Centro Internacional de Diseño del Conocimiento »Tomás Maldonado《 aufbaut - als wäre er selbst eine Faltung aus der Vergangenheit der Ulmer Hochschule für Gestaltung in unsere Gegenwart. Diese gefaltete oder synthetische Geschichte ist mein Beitrag zur interdisziplinären Gestaltung: Durch sie kann historisches Wissen zu einem notwendigen Bestandteil der Gestaltung der Zukunft werden. 


\section{Literatur}

Ahn, Bok Yeop et al. (2010): Printed Origami Structures. In: Advanced Materials, vol.22, no. 20, pp. 2251-2254.

Borges, Jorge Luis (1948): El jardin de los senderos que se bifurcan. In: ders.: Ficciones. Buenos Aires: Sur 1941.

Bréchet, Yves/Embury, J. David (2013): Architectured Materials. In: Scripta Materialia, vol.68, no. 1, pp.1-3.

Bühlmann, Vera/Hovestadt, Ludger (Hg.) (2013): Printed Physics - Matalithikum I (Applied Virtuality, 1). Wien: Springer.

Deleuze, Gilles (1988): Die Falte. Leibniz und der Barock. Frankfurt: Suhrkamp 1995

Demaine, Erik D./O'Rourke, Joseph (2007): Geometric Folding Algorithms. Linkages, Origami, Polyhedra. Cambridge: Cambridge University Press.

Dill, Ken A. et al. (2008): The Protein Folding Problem. In: Annual Review of Biophysics, vol.37, pp.289-316. Online: http://www.ncbi.nlm.nih.gov/pmc/articles/ PMC2443096/ (last access: 1 June 2015).

Felton, S. et al. (2014): Applied Origami: A Method for Building Self-Folding Machines. In: Science, vol. 345, no. 6197, pp. 664-666.

Feynman, Richard (1960): There is Plenty of Room at the Bottom. An Invitation to Enter a New Field of Physics. In: Engineering and Science, vol. 23, no. 5, pp.22-36. Online: http://calteches.library.caltech.edu/1976/1/196oBottom. pdf (last access: 1 June 2015).

Fichte, Johann Gottlieb (1794/95): Grundlage der gesamten Wissenschaftslehre. Jena.

Fratzl, Peter et al. (2011): Origami-Like Unfolding of $\mathrm{Hy}$ dro-Actuated Ice-Plant Seed Capsules. In: Nature Communications, vol. 2, p.337. Online: http://www.nature. $\mathrm{com} /$ ncomms/journal/v2/nG/full/ncomms1336.html (last access: 1 June 2015).

Fratzl, Peter/Dunlop, John W.C./Weinkamer, Richard (2013): Materials Design Inspired by Nature. Function Through Inner Architecture. Cambridge: Royal Society of Chemnistry.

Friedman, Michael (2016): Two Beginnings of Geometry and Folding: Hermann Wiener and Sunara Row. In: Journal of the British Society for the History of Mathematics.
Fuller, Richard Buckminster (1975/79): Synergetics. Explorations in the Geometry of Thinking. 2 vol. London: Macmillan. Online: https://fullerfuture. files.wordpress. com/2013/01/buckminsterfuller-synergetics.pdf (last access: 5 August 2015).

Geim, Andre K./MacDonald, Allan H. (2007): Graphene: Exploring Carbon Flatland. In: Physics Today, vol.60, no. 8 , pp. $35-41$.

Geim, Andre K./Novoselev, Konstantin S. (2007): The Rise of Graphene. In: Nature Materials, vol. 6, pp.183-191. Online: http://arxiv.org/ftp/cond-mat/papers/0702/ 0702595.pdf (last access: 26 May 2015).

Geim, Andre K./Kim, Philip (2008): Carbon Wonderland. In: Scientific American, vol.298, no.4, pp. 9o-97. Online: http://pico.phys.columbia.edu/pdf_papers/ SciAm_4_2008_PK.pdf (last access: 26 May 2015).

Helmholtz, Hermann von (1877): Über die akademische Freiheit der deutschen Universitäten: Rede beim Antritt des Rectorats an der Friedrich-Wilhelms-Universität zu Berlin am 15. October 1877. Berlin: Hirschwald 1878. Online unter: http://edoc.hu-berlin.de/ebind/hdok/ ho6_helmholtz/XML/index.xml?part=section\&division= divo\&chapter $=2$ (zuletzt aufgerufen: 1. Juni 2015).

Hooke, Robert (1665): Micrographia, or Some Physical Descriptions of Minute Bodies made by Magnifying Glasses with Observations and Inquiries Thereupon. London: Royal Society. Online: https://archive.org/details/mobot 31753000817897 (last access: 2 June 2015).

Kepes, György (Hg.) (1965): Structures in Art and in Science. New York: G. Braziller.

Kittler, Friedrich (1986): Grammophon, Film, Typewriter Berlin: Brinkmann \& Bose.

Kittler, Friedrich (1993): Draculas Vermächtnis. Technische Schriften. Leipzig: Reclam.

Krausse, Joachim/Lichtenstein, Claude (Hg.) (1999): Your Private Sky: Richard Buckminster Fuller. The Art of Design Science. Zürich: Lars Müller Publishers.

Krausse, Joachim / Lichtenstein, Claude (eds.) (2001): Your Private Sky: Richard Buckminster Fuller. Discourse. Zürich: Lars Müller Publishers. 
Landa, Manuel De (1997): A Thousand Years of Nonlinear History. New York: Zone Books.

Mahr, Bernd u.a. (2001): Mathematisch-strukturelle Grundlagen der Informatik. 2. Aufl. Berlin: Springer.

Maldonado, Tomas/Bonsiepe, Gui (1964): Wissenschaft und Gestaltung. In: ulm. Zeitschrift der Hochschule für Gestaltung, Jg.10, Nr.11, S. 10-29.

Martinez et al. (2012): Elastomeric Origami: Programmable Paper-Elastomer Composites as Pneumatic Actuators. In: Advanced Functional Materials, vol. 22, no. 7 , pp. $1376-1384$.

Monge, Gaspard (1798): Géométrie descriptive. Paris: Baudouin.

Müller, Martin (in production, 2016): First Species Whose Parent is a Computer - Synthetic Biology as Technoscien$c e$, Colonizing Futures, and The Problem of the Digital. In: Hagen, Kristin/Engelhard, Magret/Toepfer, Georg (ed.): Ambivalences of Creating Life. Societal and Philosophical Dimensions of Synthetic Biology. Berlin: Springer.

Petroski, Henry (1990): The Pencil. A History of Design and Circumstance. New York: Alfred A. Knopf.

Prusinkiewicz, Przemyslaw/Barbier de Reuille, Pierre (2010): Constraints of Space in Plant Development. In: Journal of Experimental Botany, vol.61, no.8, pp. 2117-2129.

Rinker, Dagmar u.a. (Hg.) (2003): ulmer modelle - modelle nach ulm. Hochschule für Gestaltung Ulm 1953-1968. Stuttgart: Hatje Cantz.

Rothemund, Paul W. K. (2006): Folding DNA to create nanoscale shapes and patterns. In: Nature, vol.440, pp. 297-302. Online: http://www.nature.com/nature/ journal/v440/n7082/fig_tab/nature04586_F1.html (last access: 6 July 2015).

Row, Sundara T. (1893): Geometric Exercises in Paper Folding. Ed. by Wooster W./Smith, David E. Chicago/ London: The Open Curt Pub. Company 1917. Online: https://archive.org/details/tsundararowsgeooorowrich (last access: 17 June 2015).
Schäffner, Wolfgang (2010): »The Design Turn. Eine wissenschaftliche Revolution im Geiste der Gestaltung «. In: Mareis, Claudia (Hg.): Entwerfen - Wissen - Produzieren. Designforschung im Anwendungskontext. Bielefeld: transcript, S. $33-46$.

Schäffner, Wolfgang (2016): Punkt 1.o Zur Geschichte des analogen Codes in der Frühen Neuzeit. Berlin/Zürich: diaphanes.

Serres, Michel (1968): Le système de Leibniz et ses modèles mathématiques. tome I: Étoiles, tome II: Schémas - Point. Paris: Presses Universitaires de France.

Serres, Michel (1992): Aufklärungen. Fünf Gespräche mit Bruno Latour. Berlin: Merve 2008.

Smith, Lloyd M. (2006): Nanostructures: The Manifold Faces of DNA. In: Nature, vol. 440, no. 7082, pp. 283-284.

Uversky, Vladimir (2010): The Mysterious Unfoldome: Structureless, Underappreciated, Yet Vital Part of Any Given Proteome. In: Journal of Biomedicine and Biotechnology, vol. 2010. Online: http://dx.doi.org/10.1155/ 2010/568068 (last access: 1 June 2015).

Watrous, James (1957): The Craft of Old Master Drawings. Madison: University of Wisconsin Press.

Wiener, Hermann (1890): Zur Theorie der Umwendungen. In: Berichte über die Verhandlungen der Königlich Sächsischen Gesellschaft der Wissenschaften zu Leipzig. Mathematisch-Physische Classe, Nr. 42, S. 71-87.

Yockey, Hubert P. / Platzman, Robert L. / Quastler, Henry (eds.) (1958): Symposium on Information Theory in Bio$\log \gamma$. New York/London/Paris: Pergamon Press.

Zwick, Carola et al. (eds.) (2012): The Digital Turn. Design in the Era of Interactive Technologies. Berlin: eLab. Weissensee Academy of Art. 


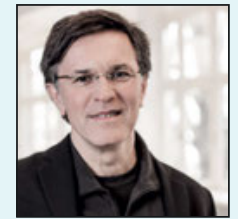

schaeffner@culture.hu-berlin.de

Principal Investigator, Sprecher

Basisprojekte: Gender \& Gestaltung, Gesundheit \& Gestaltung, Historische Strukturuntersuchungen, Strukturwissenschaft \& 3 D-Code, Architekturen des Wissen

Disziplin: Kulturwissenschaft

Wolfgang Schäffner, Wissenschafts- und Medienhistoriker, ist seit 2009 Professor für Wissens- und Kulturgeschichte an der Humboldt-Universität zu Berlin, seit 2012 Sprecher des Exzellenzclusters Bild Wissen Gestaltung und seit 2013 Direktor des Hermann von Helmholtz-Zentrums für Kulturtechnik. Seit 2005 wirkt er als Profesor invitado permanente und Direktor des Walter Gropius Forschungsprogramms an der Universidad de Buenos Aires. Zudem ist er Mitglied des wissenschaftlichen Beirats des Masterprogramms Teoría del Diseño Comunicacional der Universidad de Buenos Aires und des Masterprogramms Ciencia, Cultura y Tecnología der Universidad Autónoma de Madrid. 


\section{Abbildungsnachweis}

\section{Bilderwissen}

Bredekamp: Bilderwissen im Zeitalter des Neomanierismus

Abb. 1 Leeflang et al. 2003, 245, Abb. 85

Abb. 2 (C) VG-Bild-Kunst, Bonn 2015

Abb. 3 Torriti 1969, Taf. XX

Abb. 4, 5 (C) VG-Bild-Kunst, Bonn 2015

Abb. 6 Foto: Katrin Binner 2015

Abb.7 Bild Wissen Gestaltung 2015

Abb. 8 Jamnitzer 1568, H IV

Abb. 9 Mette 1995, 49, Abb. 43. Foto: Dr. Eckart Pott | OKAPIA

Abb. 10 Foto: Barbara Herrenkind (li.)/Aila Schultz (re.) 2014

Abb. 11 Maier 1618, 17, Emblem 2

Abb. 12 (c) Cold Spring Harbor Laboratory Press

Friedrich/Picht/Queisner/Roethe: Im Bildlabor der Neurochirurgie. Ein interdisziplinärer bildgeführter Fallbericht Abb. 1-4, 6, 8 Screenshot: Thomas Picht | Charité Berlin 2014

Abb. 5 Video Still: Nexstim Plc. 2014

Abb.7 Screenshot: Brainlab 2014

Leinfelder/Hamann/Kirstein: Wissenschaftliche Sachcomics. Multimodale Bildsprache, partizipative Wissensgenerierung und raumzeitliche Gestaltungsmöglichkeiten

Abb. 1 C Studio Nippoldt | Verlagshaus Jacoby \& Stuart, Berlin 2013

Abb. 2 (C) Martyna Zalalyte | Deutsches Museum 2014

Abb. 3 C Maki Shimizu, Ruohan Wang, José Aguiar | Bild Wissen Gestaltung 2015

Abb. 4 @ Alexandra Hamann, Elyon's | Bild Wissen Gestaltung 2015

Seliger/Jirikowski/Scholtz: Morphologische Analysen vegetabiler Ornamente der Gotik. Eine interdisziplinäre Annäherung an kunsthistorische Reihen

Abb. 1 Grafik: Babette Wiezorek | Bild Wissen Gestaltung 2014

Abb. 2 Strohmayer 2004, 47

Abb. 3 Grafik: Babette Wiezorek | Bild Wissen Gestaltung 2014

Abb. 4, 5 Grafik: Günther Jirikowski | Bild Wissen Gestaltung 2014

Abb. 6, 7 Foto: Anja Seliger | Bild Wissen Gestaltung 2014

Godau/Gaschler: Wahrnehmung von Datengrafiken. Ein verzerrter Eindruck?

Abb. 1 Grafik: Claudia Godau | Bild Wissen Gestaltung 2014

\section{Wissensstrukturen}

Hansmann/Koval: Die Entdeckung einer wissensarchitektonischen Karte

Abb. 1 Collage: Sabine Hansmann | Bild Wissen Gestaltung 2014

Abb. 2 Foto: Benjamin Meurer | Bild Wissen Gestaltung 2014

Stein: Im Umkreisen begriffen. Über die Produktivität kommunikativer Ambiguität

Abb. 1-7 Grafik: Christian Stein | Bild Wissen Gestaltung 2014 
Dürfeld: mauern. Wissensstrukturen und Strukturwissen der Architektur

Abb.1-3 (C) Universitätsbibliothek Heidelberg 2014

Abb. 4 Foto: Gramazio Kohler Architects 2006

Abb. 5 Foto: Gramazio Kohler Research | ETH Zürich 2008

Abb. 6, 7 Foto: François Lauginie 2011

Wendler: Zu einer Unschärferelation der Modelle. Präzision und Produktivität mehrdeutiger Modelle in der Gestaltung

Abb. 1 (C) The Trustees of the British Museum

Abb. 2 Zeichnung: Reinhard Wendler | Bild Wissen Gestaltung 2014

Abb. 3 C Musée Carnevalet - Histoire de Paris, Paris 2015

Abb. 4 Levinthal 1966, 42. Reproduced with permission. (C) 2015 Scientific American, Inc. All rights reserved

\section{Gestaltung als Synthese}

Oswalt: Wissen - Nichtwissen - Entwerfen

Abb. 1 Foto: Sergio Pirrone 2014

Abb. 2-5 Patente: Deutsches Patent- und Markenamt

Abb. 6 (C) Cedric Price Fonds. Collection Centre Cannadien d'Architecture | Canadian Centre for Architecture, Montréal

Abb. 7 Foto: OMA, Rotterdam

Hoffmeister: Entwerfen - Verwerfen. Ein reflektierender Werkstattbericht aus dem Interaction Design

Abb. 1-5 Foto: Anouk-Aimée Hoffmeister | Bild Wissen Gestaltung 2014

Abb. 6 Grafik: Anouk-Aimée Hoffmeister | Bild Wissen Gestaltung 2014

Kassung/Schwesinger/Seifert: Mit den Haaren hören. 1832-2014-1897

Abb. 1 Hengler 1832, Bildtafel

Abb. 2 Zöllner 1871, Bildtafel

Abb. 3 Reprint Courtesy of International Business Machines Corporation (IBM). (C) IBM

Abb. 4 (C) Free Software Foundation, Inc. 2008

Abb. 5, 6 Grafik: Christian Seifert | Bild Wissen Gestaltung 2014

Abb. 7 Koenig 1872, Bildtaf. III, Fig. 8

Abb. 8 Marichelle 1897, Bildtaf. 9a

Bock von Wülfingen: Bilder der Bewegung. Sexualität im Zellkern um 1900 und nach 2000

Abb. 1 Hertwig 1875, Anhang XI

Abb. 2 Stephens 1905, Anhang, Taf. I, fig. 1-14. (C) Carnegie Institution for Science

Abb. 3 BlueGnome 2011, 3

Schäffner: Interdisziplinäre Gestaltung. Einladung in das neue Feld einer Geistes- und Materialwissenschaft

Abb. 1, 2 Geim/Novoselev 2007, fig. 1-2. Reprinted by permission from Macmillan Publishers Ltd. (C) 2007

Abb. 3 Watrous 1957. (C) 1957 by the Regents of the University of Wisconsin System. Reprinted by permission of the University of Wisconsin Press (obere Abb.); Petroski 1990, 62. C Berol USA (untere Abb.)

Abb. 4 Hooke 1665,2 , Schem. 2

Abb. 5 Prusinkiewicz / Barbier de Reuille, 2122, fig. 6. (C) American Society of Plant Biologists. Reprinted with permission of Enrico Coen I John Innes Centre, Norwich (A, D); Utpal Nath (B, C); Yuval Eshed (E); Przemyslaw Prusinkiewicz, Pierre Barbier de Reuille (F)

Abb. 6 Row 1893, 10, 13

Abb.7 Rothemund 2006, fig. 1. Reprinted by permission from Macmillan Publishers Ltd. (C) 2006

Abb. 8 (C) Estate of R. Buckminster Fuller 1997

Abb. 9 Foto: Fabian Scholz | Bild Wissen Gestaltung 2015 
